\title{
PERFORMANCE ANALYSIS FOR LATERAL-LINE-INSPIRED SENSOR ARRAYS
}

\author{
Vicente I. Fernandez \\ Bachelor of Science Mechanical Engineering (2004) \\ California Institute of Technology, Pasadena, CA, USA \\ Submitted in partial fulfillment of the requirements for the degree of \\ Doctor of Philosophy \\ at the \\ MASSACHUSETTS INSTITUTE OF TECHNOLOGY \\ and the \\ WOODS HOLE OCEANOGRAPHIC INSTITUTION
}

June 2011

(c) 2011 Vicente Fernandez

All rights reserved.

The author hereby grants to MIT and WHOI permission to reproduce and to distribute publicly paper and electronic copies of this thesis document in whole or in part in any medium now known or hereafter created.

Signature of Author

Joint Program in Mechanical Engineering/Applied Ocean Science and Engineering Massachusetts Institute of Technology and Woods Hole Oceanographic Institution May 20, 2011

Certified by

Michael S. Triantafyllou

Thesis Supervisor

Accepted by

James C. Preisig Chair, Joint Committee for Applied Ocean Science and Engineering Massachusetts Institute of Technology Woods Hole Oceanographic Institution

Accepted by

David E. Hardt Chairman, Departmental Committee on Graduate Students Massachusetts Institute of Technology 


\title{
Performance Analysis for Lateral-Line-Inspired Sensor Arrays
}

\author{
by \\ Vicente I. Fernandez \\ Submitted to the Department of Mechanical Engineering \\ on May 20, 2011, in partial fulfillment of the \\ requirements for the degree of \\ Doctor of Philosophy
}

\begin{abstract}
The lateral line is a critical component of the fish sensory system, found to affect numerous aspects of behavior including maneuvering in complex fluid environments, schooling, prey tracking, and environment mapping. This sensory organ has no analog in modern ocean vehicles, despite its utility and ubiquity in nature, and could fill the gap left by sonar and vision systems in turbid cluttered environments. Yet, while the biological sensory system suggests the broad possibilities associated with such a sensor array, nearly nothing is known of the input processing and what information is available via the real lateral line. This thesis demonstrates and characterizes the ability of lateral-line-inspired linear pressure sensor arrays to perform two sensory tasks of relevance to biological and man-made underwater navigation systems, namely shape identification and vortex tracking.

The ability of pressure sensor arrays to emulate the "touch at a distance" feature of the lateral line, corresponding to the latter's capability of identifying the shape of objects remotely, is examined with respect to moving cylinders of different cross sections. Using the pressure distribution on a small linear array, the position and size of a cylinder is tracked at various distances. The classification of cylinder shape is considered separately, using a large database of trials to identify two classification approaches: One based on differences in the mean flow, and one trained on a subset which utilizes information from the wake. The results indicate that it is in general possible to extract specific shape information from measurements on a linear pressure sensor array, and characterize the classes of shapes which are not distinguishable via this method.

Identifying the vortices in a flow makes it possible to predict and optimize the performance of flapping foils, and to identify imminent stall in a control surface. Vortices in wakes also provide information about the object that generated the wake at distances much larger than the near-field pressure perturbations. Experimental studies in tracking a vortex pair and an individual vortex interacting with a flat plate demonstrate the ability to track vortices with a linear pressure sensor array from both small streamlined bodies and large flat bodies. Based on a theoretical analysis, the relationship between the necessary array parameters and the range of vortices of interest is established.
\end{abstract}

Thesis Supervisor: Michael S. Triantafyllou Title: Professor of Mechanical and Ocean Engineering 


\section{Acknowledgments}

First of all, I would like to gratefully acknowledge my advisor Prof. Michael Triantafyllou

for his support, insight, and guidance on this work. I would like to also acknowledge Prof. Jeffrey Lang for his advice and aid, especially on the topics of electrical experimental design and noise reduction. Prof. Franz Hover I would like to acknowledge for his direct and insightful questions as well as his advice, which frequently contributed to keeping this project well grounded. I would like to acknowledge several towing tank graduate students and post-docs that helped in experimental work or by lending moral support: Stephen Licht, Jason Dahl, Brad Simpson, Pradya Prempraneerach, and Stephen Hou. And last, but not least, I would like to acknowledge Natasha Blitvic, a keen probabilist who also happens to be my wife, for her persistent support and stimulating discussions.

I would also like to acknowledge the support of the NOAA's Sea Grant, the SingaporeMIT Alliance for Research and Technology, and the National Defense Science and Engineering Graduate (NDSEG) Fellowship for their support throughout my thesis work. 


\section{Contents}

$\begin{array}{llr}1 & \text { Introduction } & 27\end{array}$

1.1 Research Motivation . . . . . . . . . . . . . . . . 27

1.2 Chapter Overviews . . . . . . . . . . . . . . . . . . . . 32

2 Biological Foundation $\quad 35$

$2.1 \quad$ Lateral Line Structure . . . . . . . . . . . . . . . . . . . . 35

2.1.1 Superficial Subsystem . . . . . . . . . . . 36

2.1.2 Canal Subsystem . . . . . . . . . . . . . . 38

2.2 Lateral-Line-Mediated Fish Behavior . . . . . . . . . . . . . . 40

2.2.1 Approach to Lateral Line Modeling . . . . . . . . . . . . . . 41

2.2.2 The Lateral Line and Dipole Stimuli . . . . . . . . . . . . . . 42

2.2.3 The Lateral Line and Solid Objects . . . . . . . . . . . . . . 43

2.2.4 The Lateral Line and Vortices . . . . . . . . . . . . . . 45

2.3 Conclusions . . . . . . . . . . . . . . . . . 47

3 Tracking a Cylinder in Motion 449

3.1 Strategy and Motivation . . . . . . . . . . . . . 50

3.2 Model Evaluation and Selection . . . . . . . . . . . . . . 51

3.2.1 General Model Form and State Transition . . . . . . . . . . . . 52

3.2.2 Measurement Models . . . . . . . . . . . . . . . 53

3.2.3 Measurement Model Frame Equivalence . . . . . . . . . . . 55

3.3 Experimental Setup . . . . . . . . . . . . . . . . 61

3.3.1 Cylinder Motion ................... 61 


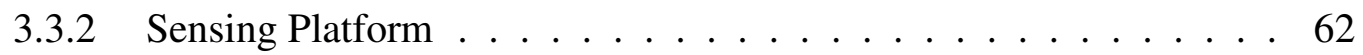

3.3 .3 Test Matrix . . . . . . . . . . . . . . . . . . . 65

3.3.4 Noise Analysis and Data Preparation . . . . . . . . . . . . 65

3.4 Results . . . . . . . . . . . . . . . . . . . . . . 68

3.5 Cylinder State Estimation . . . . . . . . . . . . . . . . . 70

3.5.1 Cylinder Tracking via a Kalman Filter . . . . . . . . . . . . 71

3.5.2 Cylinder Tracking Via Particle Filter . . . . . . . . . . . . 77

3.6 Discussion . . . . . . . . . . . . . . . . . . . . . . . 90

4 Object Identification and Classification $\quad 95$

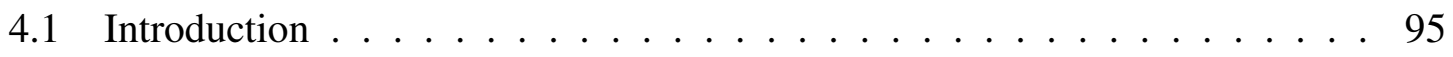

4.2 Experimental Procedure . . . . . . . . . . . . . . . 96

4.2 .1 Experimental Layout . . . . . . . . . . . . . . . . . 98

$4.2 .2 \quad$ Test Matrix . . . . . . . . . . . . . . . . . . . . . . . . . . . . . 99

4.2.3 Data Pre-Processing . . . . . . . . . . . . . . 100

4.3 Results . . . . . . . . . . . . . . . . . . . . . 102

4.4 Analysis . . . . . . . . . . . . . . . . . . . . . . 109

4.4.1 Heuristically Selected Features . . . . . . . . . . . . . . . . 109

4.4.2 Principal Component Analysis . . . . . . . . . . . . . . 112

4.4.3 Discussion and Comparison . . . . . . . . . . . 115

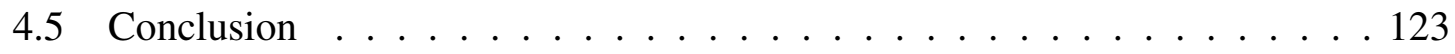

5 Vortex Tracking 127

5.1 Introduction . . . . . . . . . . . . . . . . . . . . 127

$5.1 .1 \quad$ Variable Definitions . . . . . . . . . . . . . . . 129

5.1 .2 Vortex Modeling . . . . . . . . . . . . . . . 130

5.2 Experimental Procedure . . . . . . . . . . . . . . . . . 131

$5.2 .1 \quad$ Particle Image Velocimetry . . . . . . . . . . . . . . . 132

5.2.2 Experimental Layout: Streamlined Body _ . . . . . . . . . 132

5.2 .3 Experimental Layout: Flat Body . . . . . . . . . . . . . 136

5.3 Data Processing . . . . . . . . . . . . . . . . . . . . . 139 
5.3.1 Pressure Data Pre-Processing _ . . . . . . . . . . . . . . 139

5.3 .2 PIV Processing . . . . . . . . . . . . . . . . . . . . . 142

5.3.3 Independent Vortex Identification $\ldots \ldots \ldots$. . . . . . . 143

5.4 Real Time Vortex Tracking . . . . . . . . . . . . . . . . . . . . 147

5.4.1 Experimental Vortex Forward Models . . . . . . . . . . . . 148

5.4 .2 Estimation Methods . . . . . . . . . . . . . . . . 158

5.4.3 Streamlined Body Results _ . . . . . . . . . . . . . 163

5.4 .4 Flat Plate Results . . . . . . . . . . . . . . . . . . . . . . 178

5.5 Vortex Tracking Dependence on Array Parameters . . . . . . . . . . . 184

5.5.1 Reflected Vortex Model _ . . . . . . . . . . . . . . . 185

5.5.2 Vortex Pair Model . . . . . . . . . . . . . . . . . . . . . . 194

5.6 Discussion . . . . . . . . . . . . . . . . . . 200

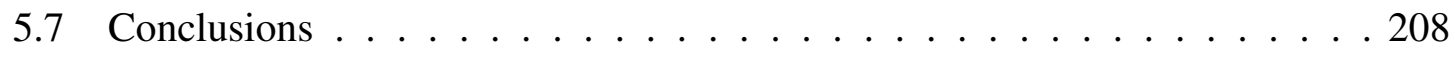

6 Conclusions 211

6.1 Overview . . . . . . . . . . . . . . . . . 211

6.2 Principal contributions of the thesis $\ldots \ldots \ldots \ldots \ldots \ldots \ldots \ldots \ldots$

6.2 .1 Passive cylinder tracking . . . . . . . . . . . . . . 212

6.2.2 Passive cylinder shape classification . . . . . . . . . . . . . 214

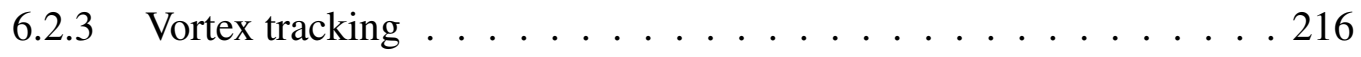

6.3 Recommendations for future work . . . . . . . . . . . . 218

6.3.1 Improved sensor array density for multiple vortex tracking . . . . 219

6.3 .2 Vortex street tracking . . . . . . . . . . . . . . . . . . 219

6.3.3 Numerical forward models for wakes _ . . . . . . . . . . 219

6.3.4 Biological object discrimination . . . . . . . . . . . 220

6.3.5 Active object identification . . . . . . . . . . . . . . 220

6.3.6 Two-dimensional sensor arrays . . . . . . . . . . . . . . 220

6.3.7 Local flow models for large objects . . . . . . . . . . . . 221 


\section{List of Figures}

1-1 Photograph of Finnegan the Roboturtle, a flapping foil autonomous underwater vehicle, undergoing tests in a pool. . . . . . . . . . . . . 30

2-1 Depiction of the canal subsystem of the lateral line at several stages of detail. Image adapted from Coombs [13] and Gibbs [28] . . . . . . . . 37

2-2 Comparison of varieties of cephalic canal lateral lines (left four images) and truck canal lateral lines (right four images). The canals are highlighted in red. Image modified from Gibbs $[28] \ldots \ldots$

3-1 Hidden Markov model undirected graph. The diagram describes the relation between a series of random variables with edges denoting direct dependence between the random variables. . . . . . . . . . . 52

3-2 Model wake boundaries. The wakes for each model are superposed around the outline of the actual cylinder for comparison. The wakes are depicted near a wall and in open water with the solid black line denoting the location of the wall. . . . . . . . . . . . . . . . . . . . . 57

3-3 Pressure comparison between models and CFD simulation in open water. The simulated CFD pressure over a ghost sensor array is displayed at regular intervals over multiple shedding periods in blue. The mean of these simulated pressure traces (in black) is compared to the results from the analytical models. . . . . . . . . . . . . . . . . . 58 
3-4 Pressure comparison between models and CFD simulation near a wall. The CFD results are displayed in blue at various intervals of the vortex shedding period. This graph corresponds to the layout described in Figure 3-2. The results of the Rankine half-body and the source-dipole hybrid model are plotted both with and without the wall present. . . . . . . . . . . . . . 60

3-5 Cylinder tracking experimental setup. A: The tank in which the experiments were performed. B: Top-down diagram of the linear sensor array setup. The coordinate system for the experiments and models is shown, with the origin at the first sensor and the $\mathrm{X}$ direction parallel to the linear array. C: Detail of the positioning system from the rear. The crosspiece is visible with the stage that forms the point of connection for the cylinder. . . 63

3-6 Amplification circuit diagram. This schematic describes the amplification circuitry module for a single pressure sensor. The arrows at the right of the diagram indicate the signal out to data acquisition. In implementation, all the sensors were powered and amplified by the same set of batteries. . . . . 64

3-7 Sensor noise characterization. A: Histogram of noise over two seconds sampled at $30 \mathrm{kHz}$ with best-fit Gaussian distribution. B: Noise power

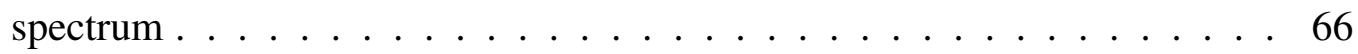

3-8 Noise processing. The pressure data is oversampled to avoid aliasing electrical noise, then antialiased and downsampled. A notch filter then removes the effect of cylinder vibrations. To avoid contamination from large wavelength water surface oscillations, the difference between adjacent pressure sensors is calculated. . . . . . . . . . . . . . . .

3-9 Averaged experimental results. These results are averaged over five runs for each point in the test matrix. All but the spatial difference step in the noise processing has been applied. . . . . . . . . . . . . 
3-10 Extended Kalman filter cylinder tracking simulation. The result from the estimation of the cylinder state based on data generated with the same model used in the Kalman filter. The top figure compares the estimated state (in color) to the true state (dotted black). The bottom shows the simulated pressure data that the extended Kalman filter is acting on. . . . . . 75

3-11 Extended Kalman filter cylinder tracking on experimental data. This figure is in the same format as Figure 3-10, but the data which the extended Kalman filter is using for the tracking was gathered from one of the experiments. Note the three forced corrections in the $\mathrm{Y}$ estimate. This result is representative of the extended Kalman filter when applied to other experimental data. . . . . . . . . . . . . . . . . . . 76

3-12 Non-Gaussian State Variable Distributions. The lognormal distribution and a truncated Gaussian distribution, corresponding to the y position and radius respectively, are shown at left for various parameter values. Their joint distribution is shown at right. . . . . . . . . . . . . . . 79

3-13 Results from particle filter on simulated data. The estimated probability distribution based on the history of measurements is displayed for each timestep for the four state variables. Each vertical slice is therefore normalized to sum to 1 . The black line denotes the expected value of the variable as calculated from the state estimate distribution. The red line is the true state used to generate the pressure measurement data. . . . . . . . 82

3-14 Simulated pressure corresponding to the particle filter estimate. This pressure difference time history is generated using the pressure measurement model based on a Rankine half-body following a state path that follows the state transition equations. No noise is included in the measurement or the state transition, resulting in a test case that offers the best conditions for

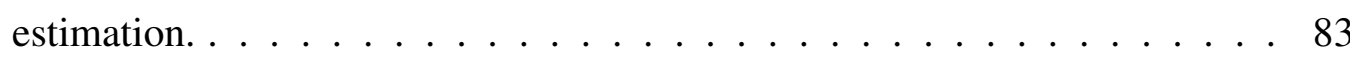


3-15 Particle filter estimation on two experimental data sets. The estimated results are shown for high and low signal strength examples from the experimental test matrix. The format of the state estimates is the same as for Figure 3-13. The corresponding pressure difference data is shown for reference against the estimate behavior. . . . . . . . . . . . 86

3-16 The dependence of error bias on particle number. The absolute average error, calculated on the time when the pressure difference magnitude is above $10 \mathrm{~Pa}$ after some initial convergence time decreases as the number of particles used increases. Each point is an average of 10 different runs of the particle filter. . . . . . . . . . . . . . . . . 88

3-17 The dependence of error standard deviation on particle number. The standard deviation in the average error, calculated over the same period of time as for Figure 3-16. The error standard deviation reaches a steady state with respect to the number of particles earlier than the error bias. . . . . . . . . . 89

3-18 Particle filter error landscape. The dependence of each state variable estimate against the speed and distance of a moving cylinder using a particle filter based on a Rankine half-body model is shown. Each point corresponds to the averaged absolute error based on five experimental runs. . . . 90

3-19 The perpendicular distance between the estimated cylinder position and the sensor array. The estimated distance is normalized by the actual distance between the closest point of the cylinder and the sensor array line. . . . . . 92

4-1 A: Schematic of experiments with cylindrical stimuli. The diagram depicts the housing with pressure ports along the side and a typical cylinder moving alongside. Several key quantities are labeled, including the separation between sensors and cylinder (s), the depth of the sensors (y), the total depth of the tank (h), the diameter of the cylinder (d), and the velocity of the cylinder (v). B: A close up of the pressure sensor and port geometry. The pressure sensors are labeled $p_{1}-p_{3}$ in the cut-out view. . . . . . . . . 97 
4-2 A: Resting noise distribution of the Honeywell 242PC15M pressure sensor. the normalized histogram is generated from a 3 second sample at $300 \mathrm{kHz}$. B: The corresponding power spectrum to the noise sample. High frequency electrical noise peaks are evident in the power spectrum. . . . . . . . . 101

4-3 Results from two sets of tests comparing pressure traces obtained from two different cross sections. Both cross sections had a diameter of two inches, velocity of $0.75 \mathrm{~m} / \mathrm{s}$, and separation of $0.51 \mathrm{~cm}(0.2 \mathrm{in})$. The mean value of all four sensors in each set of 100 runs is shown. The corresponding standard deviation is offset by $-2 \mathrm{kPa}$, but on the same scale as the mean pressure traces. . . . . . . . . . . . . . . . 103

4-4 Pressure traces for a single run with a round cylinder are depicted, showing the variability accross measurements from individual sensors. These traces were filtered with a cut-off frequency of $100 \mathrm{~Hz}$ but not aligned. The low pressure spike marked with an arrow has the signature of a vortex impinging on the sensor port. . . . . . . . . . . . . . . . . . . 104

4-5 The mean value of the pressure as function of time. Eight data sets are presented, cross-compared to assess the effect of cylinder size. Each data set is composed of 100 runs. . . . . . . . . . . . . . . 105

4-6 The mean value of the pressure as function of time. Eight data sets are presented, cross-compared to assess the effect of cylinder velocity. Each data set is composed of 100 runs. . . . . . . . . . . . . 106

4-7 The mean value of the pressure as function of time. Eight data sets are presented, cross-compared to assess the effect of cylinder shape. Each data set is composed of 100 runs. . . . . . . . . . . . . . . . 107

4-8 Diagram of selected features. The three features initially chosen for analysis are marked on a sample pressure trace. The pressure data has been filtered with a cutoff frequency of $60 \mathrm{~Hz}$. . . . . . . . . . . . . 110 
4-9 Scatterplot of individual traces according to the heuristically chosen features. Blue dots correspond to square cross sections, and red dots to round cross sections. Runs for all velocities, sizes, and separations are included. The decision line based on a sum of squared error criteria applied to a training set from one sensor is marked as a dotted black line. All 4000 datapoints from each sensor in each run are plotted. . . . . . . . . . . . 111

4-10 Coefficients for the first three principal components derived from the normalized measurements of sensor 2. These are the coefficients for the linear combinations to generate the first three principal components. Data from all experiments were used in the PCA, but only one sensor was used as a training data set. . . . . . . . . . . . . . . . . 114

4-11 Scatterplot of data from all tests and sensors according to principal components. The coefficients for the principal components were found using data from only one sensor. In blue are pressure traces from square cross sections, and in red those from round cross sections. The black plane bisecting the figure is the decision plane minimizing the sum of squared errors. 116

4-12 Histogram of the distance from the decision plane as shown in Figure 4-11. In blue is the data from the square cross section and in red from the round cross section. . . . . . . . . . . . . . . . . . 117

4-13 A: The mean pressure trace of sensor 2 from all experiments. This mean must be subtracted from any new data before classifying it with the results of the PCA. B: The coefficients for classifying a new pressure trace. These coefficients are the result of rotating the coordinates in Figure 4-11 so that one axis is normal to the decision plane. . . . . . . . . . . . . 118

4-14 Comparison of results using modified decision weights. The top subfigure indicates the division of the decision weight coefficients into different regions. A classification test using weights composed of the original coefficients with regions zeroed out is shown in the lower subfigure. The non-zero regions are labeled for each bar. . . . . . . . . . . . . . . . . 121 
5-1 Depiction of the experimental layout for tracking a vortex pair near a streamlined body. The red box marks the imaged region for PIV, which is lit by the laser in green. The location of the sensors are marked by red dots. . . . 133

5-2 Simulation of the path of counter-rotating vortices of equal magnitude near a wall. Dots are placed at equal time intervals along the path to depict the speed. The wall is marked by a dotted line at the $y=0$ axis. . . . . . . . 135

5-3 Depiction of the experimental layout for tracking a vortex pair near a flat plate. The red box marks the imaged region for PIV, which is lit by the laser in green. The location of the sensors are marked by red dots. A rough approximation to the path of the vortices is also shown. . . . . . . . . 137

5-4 Typical pressure data set from streamlined body experiments. The surface wave effects are clearly visible. . . . . . . . . . . . . . . . 14

5-5 Depiction of the results using the optimization of an analytical model to identify the state of a vortex pair. The tracking results (solid colored dots) are overlaid on an intermediate frame and corresponding velocity field. . . . 144

5-6 Comparison of the estimated state of a vortex based on PIV vector fields. Results for the vortex closest to the sensor body of a vortex pair are shown. In black filled dots are the estimates based on a least-squares fit using an analytical vortex pair model. In open colored circles are directly identified estimates based on the location of the peak vorticity and area vorticity integrals. . . . . . . . . . . . . . . . . 146

5-7 A general model for the two vortices near the streamlined body. . . . . . . 149

5-8 Reconstructed pressure from different forward models based on PIV vortex states for streamlined body experiments. The raw measured pressure is plotted in color. The thin black lines represent the reconstructed pressure predicted by the forward models. . . . . . . . . . . . . . 153

5-9 Reconstructed pressure differences from different forward models based on PIV vortex states for streamlined body experiments. The raw measured pressure is plotted in color. The thin black lines represent the reconstructed pressure predicted by the forward models. . . . . . . . . . . . . . . . 154 
5-10 A general model for a single vortex near sensors mounted on a flat plate. The dotted line represents the plane of the plate. . . . . . . . . . . 155

5-11 Reconstructed pressure differences from a forward model with a single vortex and its image based on PIV vortex states for the flat plate body experiments. The raw measured pressure is plotted in color. The thin black lines represent the reconstructed pressure predicted by the forward model. . . . . 157

5-12 Simulated extended Kalman filter application for tuning internal parameters. The upper subfigure plots the simulated pressure (dotted black line) and the reconstructed pressure (color) based on the Kalman filter state estimate. The lower subfigure displays the convergence of the state estimate (color) to the true state (dotted black line) . . . . . . . . . . . 162

5-13 Results of a streamlined body vortex estimation trial. The estimated state variables (black line) are compared against the values from the PIV data in the upper left. In the upper right, the position traces of the pressureestimated (black points) versus PIV-estimated (red points) vortices are shown against the background of the vorticity distribution at an intermediate time. The edge of the sensor body and sensor locations are marked at the left edge of the image. At the bottom, the measured pressure is shown, filtered with a $20 \mathrm{~Hz}$ cutoff frequency. . . . . . . . . . . . . . . . . 164

5-14 Results of a streamlined body vortex estimation trial. The results correspond to the same data as Figure 5-13. The position estimate based on the PIV data (red circle) is compared against that based on the pressure data only (black circle) at different stages in the time series. Each of the smaller subfigures, proceeding vertically from the top left, corresponds to the time marked by a dotted line in the top subfigure. . . . . . . . . . . . 166 
5-15 Results of a streamlined body vortex estimation trial. The estimated state variables (black line) are compared against the values from the PIV data in the upper left. In the upper right, the position traces of the pressureestimated (black points) versus PIV-estimated (red points) vortices are shown against the background of the vorticity distribution at an intermediate time. The edge of the sensor body and sensor locations are marked at the right edge of the image. At the bottom, the measured pressure is shown, filtered with a $20 \mathrm{~Hz}$ cutoff frequency. . . . . . . . . . . . . . . . 168

5-16 Results of a streamlined body vortex estimation trial. The results correspond to the same data as Figure 5-15. The position estimate based on the PIV data (red circle) is compared against that based on the pressure data only (black circle) at different stages in the time series. Each of the smaller subfigures, proceeding vertically from the top left, corresponds to the time marked by a dotted line in the top subfigure. . . . . . . . . . . . . . . 169

5-17 Results of a streamlined body vortex estimation trial. The estimated state variables (black line) are compared against the values from the PIV data in the upper left. In the upper right, the position traces of the pressureestimated (black points) versus PIV-estimated (red points) vortices are shown against the background of the vorticity distribution at an intermediate time. The edge of the sensor body and sensor locations are marked at the right edge of the image. At the bottom, the measured pressure is shown, filtered with a $20 \mathrm{~Hz}$ cutoff frequency. . . . . . . . . . . . . . 171

5-18 Results of a streamlined body vortex estimation trial. The results correspond to the same data as Figure 5-17. The position estimate based on the PIV data (red circle) is compared against that based on the pressure data only (black circle) at different stages in the time series. Each of the smaller subfigures, proceeding vertically from the top left, corresponds to the time marked by a dotted line in the top subfigure. . . . . . . . . . . . . . 172 
5-19 Results of a streamlined body vortex estimation trial. The estimated state variables (black line) are compared against the values from the PIV data in the upper left. In the upper right, the position traces of the pressureestimated (black points) versus PIV-estimated (red points) vortices are shown against the background of the vorticity distribution at an intermediate time. The edge of the sensor body and sensor locations are marked at the right edge of the image. At the bottom, the measured pressure is shown, filtered with a $20 \mathrm{~Hz}$ cutoff frequency. . . . . . . . . . . . . . . 173

5-20 Results of a streamlined body vortex estimation trial. The results correspond to the same data as Figure 5-19. The position estimate based on the PIV data (red circle) is compared against that based on the pressure data only (black circle) at different stages in the time series. Each of the smaller subfigures, proceeding vertically from the top left, corresponds to the time marked by a dotted line in the top subfigure. . . . . . . . . . . . . . . 174

5-21 Results of a streamlined body vortex estimation trial. The estimated state variables (black line) are compared against the values from the PIV data in the upper left. In the upper right, the position traces of the pressureestimated (black points) versus PIV-estimated (red points) vortices are shown against the background of the vorticity distribution at an intermediate time. The edge of the sensor body and sensor locations are marked at the right edge of the image. At the bottom, the measured pressure is shown, filtered with a $20 \mathrm{~Hz}$ cutoff frequency. . . . . . . . . . . . . . 176

5-22 Results of a streamlined body vortex estimation trial. The results correspond to the same data as Figure 5-21. The position estimate based on the PIV data (red circle) is compared against that based on the pressure data only (black circle) at different stages in the time series. Each of the smaller subfigures, proceeding vertically from the top left, corresponds to the time marked by a dotted line in the top subfigure. . . . . . . . . . . . . . . 177 
5-23 Results of a vortex estimation trial with the sensors on a flat plate. The estimated state variables (black line) are compared against the values from the PIV data at the left. In the upper right, the position traces of the pressure-estimated (black points) versus PIV-estimated (red points) vortices are shown against the background of the vorticity distribution at an intermediate time. The edge of the sensor body and sensor locations are marked at the top edge of the image. At the lower right, the unfiltered measured pressure is shown. . . . . . . . . . . . . . . 178

5-24 Results of a vortex estimation trial with the sensors on a flat plate. The results correspond to the same data as Figure 5-23. The position estimate based on the PIV data (red circle) is compared against that based on the pressure data only (black circle) at different stages in the time series. Each of the smaller subfigures, proceeding vertically from the top left, corresponds to the time marked by a dotted line in the top subfigure. . . . . . . . 181

5-25 Results of a vortex estimation trial with the sensors on a flat plate. The estimated state variables (black line) are compared against the values from the PIV data at the left. In the upper right, the position traces of the pressure-estimated (black points) versus PIV-estimated (red points) vortices are shown against the background of the vorticity distribution at an intermediate time. The edge of the sensor body and sensor locations are marked at the top edge of the image. At the lower right, the unfiltered measured pressure is shown. . . . . . . . . . . . . . . . . 182

5-26 Results of a vortex estimation trial with the sensors on a flat plate. The results correspond to the same data as Figure 5-25. The position estimate based on the PIV data (red circle) is compared against that based on the pressure data only (black circle) at different stages in the time series. Each of the smaller subfigures, proceeding vertically from the top left, corresponds to the time marked by a dotted line in the top subfigure. . . . . . . 183 
5-27 Instantaneous inversion based on a single pressure sensor for the reflected vortex model. Part A shows the spatial map of the cost function generated with one sensor. The small $\mathrm{x}$ at $(0,0.1)$ denotes the true location of the vortex. Part B depicts the pressure distribution on the wall at the $y=0$ axis. 187

5-28 Maps of the cost function for a reflected vortex model with the vortex at various lateral locations. The $\mathrm{x}$ in each image with a y position of 0.1 and an $x$ position labeled in the subfigure title denotes the true location of the vortex. The black lines correspond to level sets generated by the assumption of noise with standard deviations of 1,5 , and $10 \%$ of the maximum measured pressure. . . . . . . . . . . . . . . . 190

5-29 Maps of the cost function for a reflected vortex model with an increasing array length but constant sensor spacing. The $\mathrm{x}$ at $(0,0.1)$ in each image denotes the true location of the vortex. The black lines correspond to level sets generated by the assumption of noise with standard deviations of 1,5 , and $10 \%$ of the maximum measured pressure. . . . . . . . . . 191

5-30 Maps of the cost function for a reflected vortex model with an increasing array length and fixed number of sensors. The $x$ at $(0,0.1)$ in each image denotes the true location of the vortex. The black lines correspond to level sets generated by the assumption of noise with standard deviations of 1,5 , and $10 \%$ of the maximum measured pressure. . . . . . . . . . 193

5-31 The pressure distribution on a linear array due to an external vortex pair. The separation between the vortices is given in the legend with respect to the perpendicular distance between the sensor array and the closest vortex. . 195

5-32 Maps of the cost function for a vortex pair model with the vortices at various lateral locations. The $\mathrm{x}$ in each image denotes the true locations of the vortices. The black lines correspond to level sets generated by the assumption of noise with standard deviations of 1,5 , and $10 \%$ of the maximum measured pressure. . . . . . . . . . . . . . . 196 
5-33 Maps of the cost function for a vortex pair model with an increasing array length but constant sensor spacing. The $\mathrm{x}$ in each image denotes the true location of the vortex. The black lines correspond to level sets generated by the assumption of noise with standard deviations of 1,5 , and $10 \%$ of the maximum measured pressure. . . . . . . . . . . . . . . . . 198

5-34 Maps of the cost function for a vortex pair model with an increasing array length fixed number of sensors. The $\mathrm{x}$ in each image denotes the true location of the vortex. The black lines correspond to level sets generated by the assumption of noise with standard deviations of 1,5 , and $10 \%$ of the maximum measured pressure. . . . . . . . . . . . . . . . . . 199

5-35 Simulated setup for a pressure sensor array similar to the one on a streamlined body being applied to a foil in order to track an external vortex. . . . . 203

5-36 Dependence of foil lift on the position of an external vortex. In the top subfigure, the foil is plotted with horizontal lines indicating the range of positions of the external vortex. The lower subfigure demonstrates the effect of a vortex at the positions marked in the top subfigure on the normalized lift of the foil. . . . . . . . . . . . . . . . . . . . . 204

5-37 Dependence of foil moment on the position of an external vortex. In the top subfigure, the foil is plotted with horizontal lines indicating the range of positions of the external vortex. The lower subfigure demonstrates the effect of a vortex at the positions marked in the top subfigure on the normalized moment of the foil. . . . . . . . . . . . . . 207 


\section{List of Tables}

3.1 Experimental matrix for cylinder tracking. The range of velocities and distances was determined by the signal strength and by the abilities and noise of the experimental setup. . . . . . . . . . . . . . 65

4.1 Table of tests performed for identifying a moving object. Not included in the table are two sets of 100 runs (one for each cross section shape) at 0.75 $\mathrm{m} / \mathrm{s}, 5.08 \mathrm{~cm}$ diameter, and $1.27 \mathrm{~cm}$ separation. . . . . . . . . . . . . 100

4.2 Table of the Strouhal numbers associated with the cylinder identification experiments. . . . . . . . . . . . . . . . . . . . . . 123

5.1 Error statistics for tracking vortices from a streamlined body. The statistics are calculated while the vortex is in front of the sensor array, allowing for a brief window of convergence. . . . . . . . . . . . . . . . . 201

5.2 Error statistics for tracking vortices from a flat plate. The statistics are calculated while the vortex is in front of the sensor array, allowing for a brief window of convergence. . . . . . . . . . . . . . . . 202 


\section{Chapter 1}

\section{Introduction}

The fish lateral line is a versatile short-range sensor organ, involved in mapping environments [76], identifying objects [75, 82], tracking prey [16, 20], and conserving energy while swimming in wakes [43]. Despite its utility in the natural underwater world, there is currently no analog to the sensor for ships or underwater vehicles. Translated to ships and autonomous underwater vehicles (AUV's), this sensory system would provide substantial benefits in maneuvering and object detection in difficult environments. Although many behaviors have been associated with the lateral line in fish, the type of processing and information extracted by the fish has yet to be discovered. It is unknown whether fish are obtaining specific information about stimuli, such as the exact location of a vortex, or whether they are simply responding to broader trends, such as the indication of the presence of a vortex on one side. The extent to which velocity measurements play a role in fish responses is also unclear. Without understanding the extent to which spatial information can be derived via the lateral line organ, it is impossible to transfer the sensor to man-made vehicles.

\subsection{Research Motivation}

The primary goal of this research is to establish whether the position, size, and shape of moving cylinders and their wake structures can be extracted in real time solely from pressure measurements linearly distributed on the surface of a submerged body. The question 
of identifying solid objects in a flow is of particular interest to underwater vehicles operating near shore, where obstacles are common and the turbid, cluttered environment are problematic for vision and sonar systems. AUV's are largely used in open volumes of water due to these limitation, but there is increasing interest in operating them in littoral zone, requiring increased maneuverability and improved sensing. For example, many underwater inspection tasks are currently still carried out by divers, despite the tedious and dangerous nature of the task. These inspection are done for maintenance and security reasons, and include structures such as oil platforms and extraction networks, bridges, and harbor pilings. These inspections are frequently necessary in areas that are regularly turbid with external currents, and the entire purpose is to inspect cluttered structures. For this reason, this thesis focuses on tracking and identifying a moving cylindrical object and it's wake based on pressure measurements emulating the fish lateral line. The wake is a significant factor in this scenario. It obscures information about the shape of the object, but it also extends the range in which the object can be detected due to the longevity of the vortices that are shed from the cylinder, overcoming the inherent range limitations of distributed pressure sensors.

The primary sensory systems available to AUVs for navigation, vision and sonar, fail in the difficult environments encountered in littoral zones and for underwater inspection. Due to the multiple targets and frequently relatively shallow water depth, sonar frequently suffers from multipath issues which lead to false positives and make navigation difficult. Similarly, the turbidity of the water directly limits the function of vision systems by the reducing the visibility range. In contrast, a sensor that functioned along the lines of the lateral line organ in fish would naturally bypass these issues. Such a sensor would naturally emphasize the closest stimuli providing both it's advantages and limitations. It would not be affected by turbidity since it does not use light, and would disregard complicated structures not in the vicinity, thus focusing on the objects necessary for immediate navigation. The caveat for such a sensor would be that its range of operation is limited by the array length and the noise floor, as discussed in Chapter 5. In addition to the differences in functionality, both vision and sonar systems are active, requiring the emission of energy, acoustic or light, into the fluid. This leads to substantial power consumption on platforms that tend to have 
a strict power budget (e.g. $20 \mathrm{~W}$ for Didson Sonar and more than 50W for Remote Ocean Systems vision system). In contrast, a lateral-line-like array is passive apart from any flow disturbances caused by the motion of the AUV. The comparable power consumption depends on the exact parameters of the sensor array, but would be on the order of $0.7 \mathrm{~W}$, more than an order of magnitude smaller than the traditional systems. This makes a lateralline-like sensor array an attractive addition for AUVs working in difficult environments, potentially providing detailed aid in navigation with little additional cost.

The identification of vortices is of significant interest on its own merit, apart from what it implies about solid bodies in the flow. The pressure field generate by one or several vortices is what allows their detection from a lateral-line-like sensor, but it also affects the operation of any nearby control surface or foil. This is particularly true in the cases when the vortices are generated on the surface of interest, for example when the angle of attack of a rudder is too large or during the normal operation of a flapping foil. In these cases, the location and strength of the vortex affect the performance of the structure by altering the lift and drag characteristics. Figure 1-1 shows a vehicle designed to use four flapping foils for highly maneuverable locomotion (see $[47,86]$ ). In this configuration, the rear pair of foils operate directly in the wake of the front pair, and therefore frequently interact with shed vortices. While a uniform steady flow maybe an adequate flow model for the front foils, the rear foils operate in a more complicated environment and it is difficult to predict the lift and thrust that will be generated for any particular motion. Knowing the location of vortices as they approach and pass near the foils would greatly aid in improving the flow model for these rear foils.

Even more than on rudders, knowledge of the local vortices would be particularly useful if implemented on control surfaces that can undergo rapid changes in angle of attack. In this situation, a dynamic stall occurs which differs in a number of respects from the steady state stall frequently considered. One difference is that due to the dynamics of the flow, the angle at which stall occurs is higher than in the static case, and dependant on the rate of change of the angle of attack $[9,26]$. In addition, the final loss of lift in dynamic stall is preceded by a strong leading edge vortex that travels over the surface of the foil. These features make detecting imminent dynamic stall a promising flow monitoring application for 
vortex detection with a lateral-line-like sensor array. One particular example in which this could be applied is the roll stabilization of ships. This is accomplished using a pair of foils at approximately the midpoint of the ship hull which are actuated in pitch to control against the roll of ships interested in steadier motion. The system typically works well unless a series of large waves suddenly increase the effective angle of attack, leading to dynamic stall and loss of control in roll stabilization. Current approaches to dealing with these concerns involve reducing the feedback gain when stall is detected and attempting to estimate the angle of attack based on the response to the stabilizer pitch [61]. An alternative to these global modeling approaches would be possible with the proposed lateral-line-inspired sensor array, which could detect the leading edge vortex with time to provide a corrective action before stall. This approach would allow more aggressive control under difficult sea states.

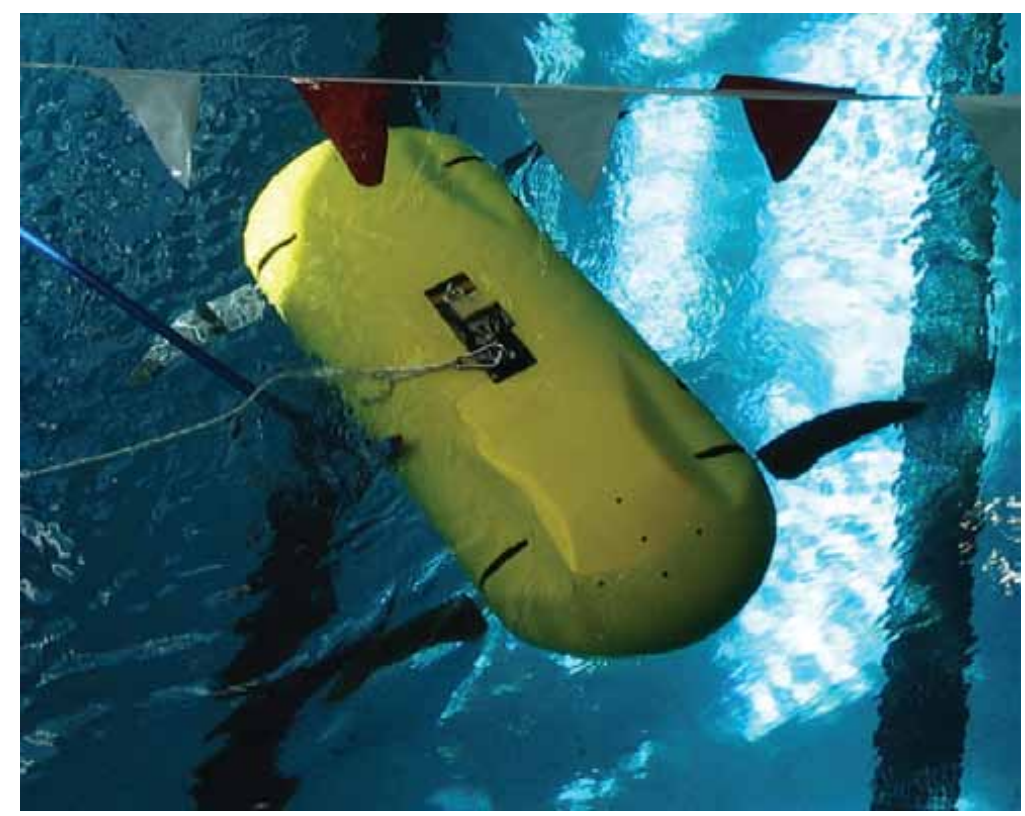

Figure 1-1: Photograph of Finnegan the Roboturtle, a flapping foil autonomous underwater vehicle, undergoing tests in a pool.

In addition, there has been interest in extracting energy from currents using a flapping foil device [89]. In this scenario, self-generated vortices are of critical importance to maximizing the lift and energy efficiency. The formation and shedding of the leading edge vortices in a flapping foil depend on the heave and pitch of the foil [71]. Monitoring the 
leading edge vortices would allow the motion parameters to be changed and optimized as external conditions vary.

Researchers have only lightly considered the topic of a lateral-line-like sensor for manmade vehicles up to this point. Much work has been done by biologists in examining the function of the lateral line and connecting it to the behaviors and abilities of the fish (see [13] and Chapter 2). However, only recently have scientists looked to translate the abilities of the lateral line for applications to man-made vehicles. These approaches have varied substantially in method, using hot wire anemometers [87] and replica hair cells [49] to recreate the measurements of the lateral line. Even then, the focus has been on identifying and tracking an oscillating dipole stimulus, which roughly models small prey for fish.

In this work, the focus on the study on inference with a lateral-line-like sensor is for applications to ships and underwater vehicles. For this reason, the lateral line is approximated by a linear array of pressure sensors. This approximates the information gathered by the trunk canal portion of the lateral line organ, which has been shown to respond to pressure gradients in fish [79], and is responsible for the majority of fish behaviors discussed so far. The one-dimensional nature of the sensor array means that there is limited information about an object perpendicular to the plane formed by a point on the object and the linear array. As a result, only stimuli that are broadly two-dimensional are used. This reduces the complexity of the problem while preserving its biological and engineering relevance.

In addition to examining the ability of an implementation of a lateral-line-like sensor array for object and vortex tracking, this thesis will consider some basic questions about the nature sensor array. In particular, the questions of whether absolute pressure measurements are sufficient in comparision to pressure gradient measurements, and whether velocity measurements are superfluous for the considered applications, will be addressed. These questions are derived from differences between the abstraction of the biological lateral line for AUV applications and the original biological sensory organ. In addition, the relationship between the parameters of a linear pressure sensor array and the accuracy of the estimation, particularly in the case of vortex tracking, will be examined. 


\subsection{Chapter Overviews}

Chapter 2 covers background information on the fish lateral line, including the basic structure and performance of the lateral line, differences between the trunk and cephalic portions of the lateral line, behaviors attributed to the lateral line, and stimuli used in research with the fish lateral line. This background further motivates the specifics of the problem addressed in this thesis of estimating the position, size, and shape of a moving cylinder and its wake based on a pressure sensor array.

Chapter 3 answers the question of whether the position and size of a moving circular cylinder can be tracked using a linear pressure sensor array. This consists of identifying a model that captures the dominant features of the moving cylinder and its wake on the pressure sensing array, experiments realizing the test problem under a number of velocities and distances, and an analysis of the results of estimation algorithms on the data.

In Chapter 4, the question of identifying the shape of a moving cylinder is addressed. A separate experimental setup is described for distinguishing a circular cross section from a square cross section, emphasizing the repeatability of the experiment with accumulating noise over precision in some of the experimental parameters. The results are considered on average, leading to a feature set that reliably classifies the moving object. The consideration of a non-parametric feature extraction method, principal component analysis, results in considerably higher classification success.

Chapter 5 considers the wake of a moving object specifically, focusing on tracking vortices using the same linear pressure sensor array. Using the experimental apparatuses from both they cylinder tracking and cylinder shape experiments, it is demonstrated that externally generated vortices can be tracked in position and strength using a linear pressure sensor array. The body one which the sensor array is mounted has a substantial effect on vortex tracking. Two types of structures are examined: a fish-like body and a hull-like body, demonstrating changes in the model that are sufficient to adapt to the different situations. In addition, results describing the relation between vortex tracking and the sensor array parameters are discussed.

Chapter 6 concludes the thesis with a discussion of the primary contributions. Sugges- 
tions for further work are also discussed. 


\section{Chapter 2}

\section{Biological Foundation}

This chapter surveys aspects of the work done in investigating the structure and function of the lateral line organ in fish. Although the lateral line organ is a complicated sensory system with multiple parts, a complete mimicry of the lateral line is unnecessary to achieve the goals of translating certain lateral-line-like abilities for AUV's and ships. The distinction between the critical and the likely superfluous components to a lateral-line-like sensor given the stated goals can only be developed via an understanding of the operation of the biological lateral line. As will be discussed, there remain significant gaps in the knowledge of the workings of the lateral line, particularly in the information processing, which will need to be overcome in order to track cylinders and vortices.

The first portion of this chapter describes the lateral line organ, discussing the structure of the two subsystems of the lateral line and their associated functionality, with the goal of identifying an appropriate analog which can be used for tracking moving cylinders and vortices. Subsequently, research into the lateral line response to three types of artificial stimuli will be discussed in detail. These stimuli are an oscillating dipole, physical objects in the environment, and vortices.

\subsection{Lateral Line Structure}

All aspects of the lateral line system rely on a single fundamental sensory element: the neuromast. The structure of the neuromast is consistent for all fish, though the proportions 
and shapes of the different elements may vary. Broadly speaking, a neuromast is a haircell-based deflection sensor that works roughly like a cantilever extended into the flow. The drag generated by the flow around the neuromast structure causes bending and translation of the extended structure which is converted into electrical signals for the fish.

A neuromast is composed primarily of three critical components: kinocilia, stereocilia, and the cupula (see $[28,51]$ for reviews). A diagram of a representative neuromast is shown in the lowest portion of Figure 2-1. In a neuromast, several hair cells together each extend a long kinocilium out from the surface. These long hairs move in response to the local flow, but fundamentally act as mechanical amplifiers. The signal transduction occurs via the short stereocilia which are connected to each kinocilium near the base. In order to extend the size of the kinocilia bundle and move them all together, the entire structure is encased in a gelatinous cupula. All together, the bundle of hair cells and cupula form the sensory element referred to as a neuromast. The resulting sensing element does not respond directly to pressure, but instead responds to flow velocity via a drag-like mechanism. Due to the relative positions and connections between the kinocilia and stereocilia, neuromasts are directional sensors flow velocity sensors. The stimulation of a neuromast with respect to flow angle roughly follows a cosine distribution [55].

In the following, we shall discuss the two subsystems of the lateral line: the superficial lateral line and the canal lateral line. While both subsystems rely on the neuromasts as the fundamental building blocks, they provide drastically different functionalities.

\subsubsection{Superficial Subsystem}

A number of neuromasts are distributed over the surface of the fish body, in configurations that greatly vary with species, forming the superficial lateral line subsystem (small dots in Figure 2-1). These neuromasts interact directly with the external flow, governing the nature of the stimuli and behaviors that associate with it. Although there are similarities between the superficial neuromasts in all fish, there is also a large amount of variability in the size, number, and distribution between different fish species, and even between different fish within a species (sighted and blind, for example) $[55,77]$. 


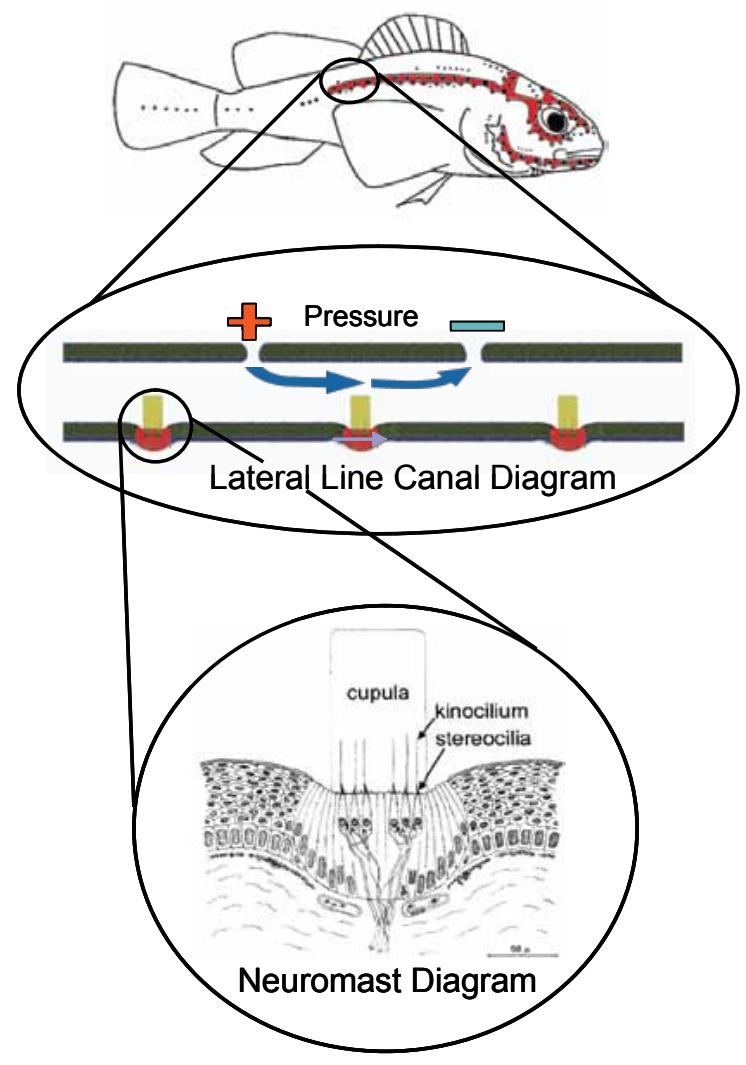

Figure 2-1: Depiction of the canal subsystem of the lateral line at several stages of detail. Image adapted from Coombs [13] and Gibbs [28]

The neuromasts in the superficial lateral line are elongated and respond to the flow velocity in the near vicinity of the fish [84], subject to alteration by the boundary layer. Besides reducing the signal amplitude, the boundary layer affects the frequency response of the superficial neuromasts, attenuating the amplitude of low frequency oscillations [51]. At high frequencies the frequency response is more strongly limited by the structural dynamics [51]. The end result is a frequency response that roughly approximates a low-pass filter [58], though the low-frequency response is not flat.

One important consequence of the position of the superficial neuromasts is their sensitivity to the steady flow component. From one perspective, this is an advantage: it is unsurprising to find that they are an important sensory component in the rheotaxis behavior of a fish, in which the fish orients itself upstream [53, 2, 55]. As with most of the experiments demonstrating a link between the lateral line and a fish behavior, this conclusion is 
reached by examining changes in behavior when the lateral line component is deactivated. The steady direct component of the flow identified for rheotaxis generates problems when attempting to identify small oscillatory stimuli such as a dipole (discussed in more detail below). In particular, the sensitivity of the superficial neuromasts to oscillatory stimuli was found to be severely degraded in the presence of steady flow [25].

It is also of interest to note that the superficial neuromasts appear to be utilized in small groups by fish. Each neuromast on the surface is not innervated individually, but a group of 5 to 10 are innervated jointly $[14,59]$. This implies that the flow velocity information is effectively averaged over a small region on the fish surface before any further processing. The averaging is likely connected to the reduced reliability of the superficial neuromasts in steady flow.

Although there remain open questions on the importance and utility of superficial neuromasts. The primary associated behavior with this subsystem of the lateral line remains rheotaxis. As such, it implies a limited utility for the purposes of a lateral-line-like sensor focused on identifying solid objects and flow structures.

\subsubsection{Canal Subsystem}

In contrast to the superficial neuromasts, the neuromasts forming the canal subsystem of the lateral line are removed from the direct external flow. These neuromasts are instead enclosed in sub-dermal canals which regularly open to the flow through pores. Between each pair of pores is a neuromast [28]. A model depiction of the canal system is shown in Figure 2-1. In the top image, the canal system of the lateral line in a representative fish is highlighted in red. Both experimentally and theoretically, it has been shown that the canal neuromasts of the lateral line respond to pressure gradients over the surface of the fish [23]. The manner in which this occurs is depicted in the middle diagram of Figure 2-1. Although the neuromast in the canal still responds to the fluid velocity, the flow in the canal is driven by pressure differences across the adjacent pores. Thus a gradient in the pressure on the surface of the fish will cause a mismatch between the pressure in adjacent pores, and stimulate in the underlying canal neuromast. Besides altering the nature of the stimulus, the 
canals also appear to have frequency-shaping properties, which compensate for the natural frequency of the neuromast to generate a fairly flat sensitivity up to $100 \mathrm{~Hz}[79,42]$.

As a result of being driven by the pressure gradient across the body of the fish, the canal neuromasts are not effected by the boundary layer on the fish body. Perhaps more importantly, the steady flow signal which saturates the superficial neuromasts is substantially reduced for the canal neuromasts. The steady flow develops a consistent pressure distribution over the surface of the fish which likely forms the baseline for perturbations due to nearby stimuli $[33,34]$.

In contrast to the superficial neuromasts, each individual canal neuromast is independently innervated [59]. This emphasis on the importance of each individual canal neuromast is also evident in the shape of the canals, which are frequently irregularly shaped in order to magnify the fluid velocity at the location of the neuromasts [28] (see lower left cephalic canal system in Figure 2-2 for example). This difference in treatment when compared to the superficial neuromasts indicates that the details of the pressure distribution are important to the functioning of the canal portion of the lateral line, as opposed to global or ensemble measurements.

The canal system in the lateral line is typically divided further into two types of canals: the cephalic (near the head) and trunk canals. These two sections of the canal system are differentiated by their location on the fish and the structure of the canals. Both types of canals are present in all fish, though there is significant variation in the form (Figure 2-2). The cephalic canal system is typically composed of several interconnected lengths on the jaw and around the eye, while the trunk canals are typically very linear and extend the length of the fish body from just behind the head to the tail [55]. The practical result from this is that the cephalic canals cover a curved surface, potentially extracting information about the three dimensional pressure gradient in the vicinity of the fish head. In contrast, the trunk canal is frequently a single linear array which can only measure the pressure gradient along the axis of the canal. Although the details of the canal system vary substantially between species, a typical inter-pore spacing of the trunk canal is $2 \mathrm{~mm}$ [17]. This corresponds specifically to the mottled sculpin species, a sit-and-wait predator with a length ranging from 7 to $9.5 \mathrm{~cm}$ [15] (which gives an upper bound to the trunk canal length). 

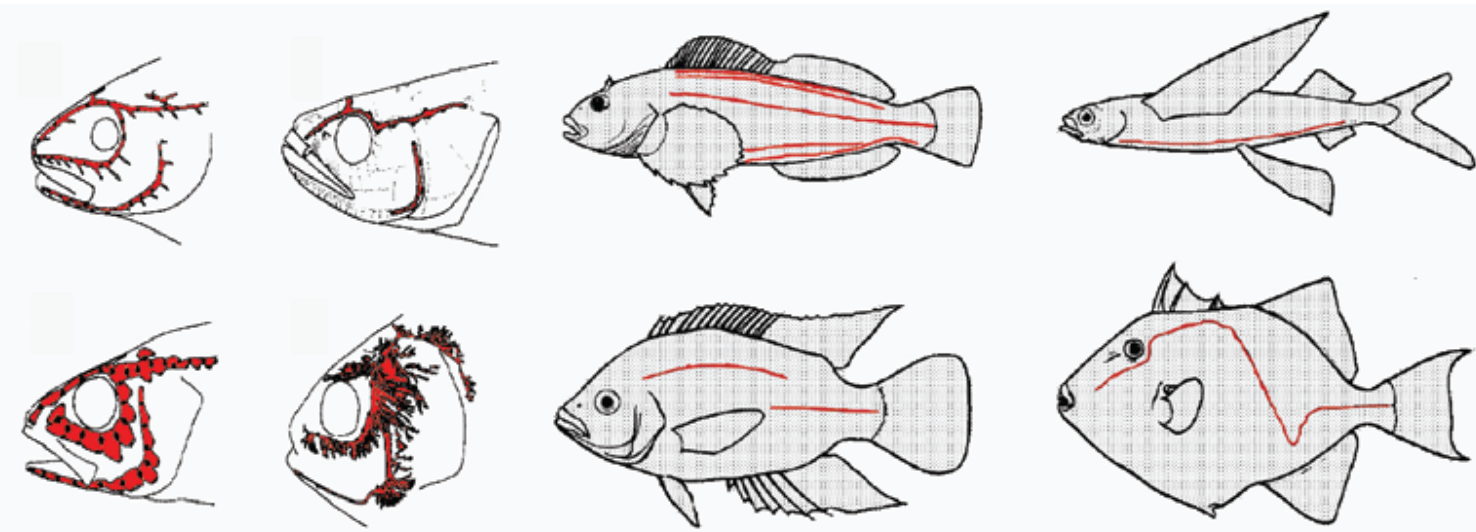

Figure 2-2: Comparison of varieties of cephalic canal lateral lines (left four images) and truck canal lateral lines (right four images). The canals are highlighted in red. Image modified from Gibbs [28]

\subsection{Lateral-Line-Mediated Fish Behavior}

Through a large number of experiments with many fish species, a number of behaviors have been definitively connected to the lateral line organ. In many of the fish behaviors, the lateral line is not necessarily the only relevant sensory input, but the removal of the lateral line results in a significant degradation or alteration of the behavior. The most frequent overlap with the lateral line sensory system is the inner ear [19, 7], but vision [43] can also play a role. For this reason, naturally blind cavefish have played a critical role in investigating the specific capabilities of the lateral line [55]. In these fish, the lateral line effectively takes the place of vision.

Among the many behaviors that have associated with the lateral line are many that fall under predator-prey relationships, including escape responses [50], plankton feeding [57], bivalve feeding [56], and ambush feeding [54]. In many of these cases, the fish that exhibit these behaviors will do so when the environment inhibits other sensory cues, such as the stargazer fish ambushing prey in the dark.

When one considers naturally blind or nocturnal fish, a number of further behaviors in locomotion are associated with the lateral line, such as collision avoidance [75], the ability to map environments $[76,8]$, and to identify detailed characteristics of different physical structures [32]. In general the strong tie between these behaviors and the lateral 
line is not as noticeable in other sighted fish. For sighted fish, there are still behaviors that cannot exclusively rely on sight and must still utilize the lateral line. These are behaviors in which the motion of the fish depends on the invisible local flow structures, such as when schooling [62] or exploiting the flow behind a rock in a stream [43]. In both of those cases, a normally sighted fish that artificially blinded is able to accomplish the task relying solely on the lateral line inputs. As already mentioned, many species of fish also utilize the lateral line for alignment with the surrounding flow [53].

A few other behaviors have been discovered to depend on the lateral line which do not fall under the subjects of underwater predator-prey relations and locomotion. For exam-

ple, certain species of salmon have been found to communicate via the lateral line during spawning [69]. In addition, some fish species are able to locate sources of disturbances in on the surface based on ripples [4].

Based on these observations, the following section outlines the general philosophy and approach to lateral line modeling taken in this thesis, followed by discussions of the three stimuli that will be considered, namely the dipole, rigid bodies, and vortices. The dipole stimulus is the most researched artificial stimulus for the lateral line, and the remaining two have distinct non-biological applications for AUV's and ocean vehicles.

\subsubsection{Approach to Lateral Line Modeling}

Of all the previously-mentioned behaviors, all but rheotaxis are either exclusively or predominantly associated with the canal neuromasts as opposed to the superficial neuromasts. For this reason, it is reasonable to interpret that pressure gradients are the primary sensory input necessary for these behaviors, as opposed to the flow velocity information obtained by the superficial neuromasts. The details of the cephalic versus trunk canal portions are less well understood, but it is likely that most of the prey tracking or locating behaviors utilize the cephalic canals. In contrast, based on the fish behavior in experiments such as those by Hassan [32], that many of the object and fluid structure discrimination behaviors rely on the trunk canals.

This thesis focuses on examining and reproducing the ability to passively detect mov- 
ing solid objects and vortices, which fall under the trunk canal behaviors. Therefore, we employ a linear array of pressure sensors to obtain the same quality of information as the trunk canal neuromasts. Although the fish lateral line is stimulated by the difference in pressure across a region, common microelectromechanical sensors (MEMS) pressure sensors are readily available to measure pressure over a large range and the pressure difference can be extracted easily from an array of these sensors. Note that there is no attempt to recreate the structure of the canal system or the neuromasts. Rather, this thesis is focused on the inference that can be accomplished based on the information available to a lateralline-like sensor array. Based on what is known of the function of the canal system of the lateral line, an array of pressure sensors will capture that information. It is possible that there are details, such as in the frequency response shaping of the canal system, which are evolutionarily optimized for lateral line behaviors in fish, but such subtleties will not be captured in an engineering approximation to the lateral line until they are well understood in fish. Also, it is possible that the canal neuromast is a repurposing of an existing sensory unit and therefore an evolutionary local minimum. For these reasons, the lateral line is an important guide and inspiration for this thesis, identifying the type of measurements to be used and the possible information to be extracted, but the thesis does not attempt to recreate or mimic the fish lateral line.

\subsubsection{The Lateral Line and Dipole Stimuli}

By far the most attention in artificial stimuli for the lateral line in fish has been devoted to the dipole stimulus (See for example $[55,57,14,18,20,21,11])$. The dipole stimulus is generated by mechanically vibrating a small bead with a sinusoidal displacement at a constant frequency, typically on the order of $60 \mathrm{~Hz}[13,21]$. The general motivation for this stimulus type is that it mimics certain aspects of invertebrate prey such as plankton [56, $57,55]$. This is primarily supported by the way in which a vibrating sphere can induce a feeding response in fish such as the mottled sculpin, where the fish will approach the sphere

and occasionally strike at the sphere [13]. A substantial amount of the preference for the dipole stimulus in lateral line experiments is likely also due to it's ease of implementation 
and simulation, since measurements of the frequency distribution of planktonic prey have shown an upper bound at approximately $50 \mathrm{~Hz}[57,56]$

However, as a result of the well-defined, repeatable stimulus, many details have been learned about the lateral line's response to a dipole. Using the feeding response of the fish, it has been possible to associate the detection of a dipole stimulus exclusively with the canal portion of the lateral line, by sequentially selectively deactivating the canal and superficial neuromasts [14]. In addition, numerous studies have established the sensitivity of the lateral line with respect to the dipole distance and frequency.

Although little is definitively known about how a fish determines the location, or at least relative angle, of a vibrating bead, recently there have been several attempts to recreate the analysis. Wavelets have been used to successfully locate a dipole in simulation, utilizing the canonical pressure distribution formed on a linear truck-like canal as the mother wavelet [21]. This approach is attractive due to its ease of implementation and potential biological implementation. Experimental approaches using a hot wire anemometer have demonstrated that it is also possible to locate a dipole using an instantaneous least-squares minimization [87].

\subsubsection{The Lateral Line and Solid Objects}

Ever since the lateral line was originally described as an organ with the ability to "touch at a distance" [24], one of the most intriguing aspects of the lateral line has been its mapping of the environment and rigid objects. This phrase has been used to describe how a fish appears able to obtain information about rigid objects in the flow without coming into physical contact. The majority of the experimental results in this area with the lateral line come from blind or nocturnal fish, which have an increased reliance on the lateral line.

The most basic level of interaction between the fish lateral line and solid objects is collision avoidance. The lateral line plays an active role in collision avoidance for blind fish [85]. Interestingly, the approach is not foolproof, and blind fish will commonly collide with a wall if they are in the middle of a tail beat as opposed to a glide phase. This has implications on self generated noise which may mask external stimuli. 
A more notable behavior associated with the lateral line in blind fish is the ability to map out a new environment using the lateral line [76]. Blind cave fish have a particular behavior when introduced into a novel environment: they swim at elevated speeds for a period of up to a day, after which they swim more slowly. This behavior appears to be an exploration and familiarization of the surroundings. If a fish is removed from such an environment, but returned to it within 2 days, it will spend significantly less time exploring it the second time. This has been taken as an indication that the fish generates a spatial map of the environment through exploration.

Experiments with in which moving objects are passed near a stationary fish have been used to examine non-oscillatory object stimuli $[52,80]$. While these experiments are very suggestive that a fish is able to extract detailed information about the shape, size, and velocity of the passing object, they cannot confirm that there is knowledge or information about the object itself as opposed to a change in the measured patters of the lateral line inputs. For example, if there was a true understanding of the passing object, then a rotation of the object should not elicit a startle response. This is the difference between accustomization to a particular lateral line input versus knowledge of the object generating that signal.

A more conclusive set of experiments was accomplished by Weissert and von Campenhausen [82] and later by Hassan [32] in which a fish was required to identify and recall a pattern of vertical bars in order to obtain food. A fish was given two choices of holes to enter, surrounded by different patters of vertical bars. The correct choice of pattern resulted in a food reward. In these experiments, the location of the bars changed in order to avoid other factors influencing memory. After some training blind fish were able to regularly identify the correct pattern by swimming past each entrance. In this experiment, the free swimming fish interrogates each bar pattern several times and remembers the correct pattern across different trials. This implies at minimum that the fish has a sense of the lateral line pattern associated with an object that can change location. Again, whether the excitation pattern is translated into a detailed model of the vertical bars is unknown. However, due to the changing location and the ability of the fish to approach the bar pattern from any direction, this experiment confirms that a fish extracts more abstract knowledge about an object than we could conclude from the moving object experiments. 
Based on all of these results, there is a substantial amount of evidence pointing to the possibility that a lateral-line-like sensor can locate and identify solid objects. However, the level of detail of the information available about the objects is unknown. For example, is it possible to distinguish all shapes? If not, what shapes are indistinguishable and how much information can be extracted about them? Given the interest in using a lateral-line-like array for filling the gap left by vision and sonar systems in complex environments and the lack of answer from studies of the fish lateral line, it is important to address these questions directly.

The biological experiments discussed on object identification have fallen into two categories: active and passive object detection. This thesis focuses on the passive detection of objects, in which the stimulus is generated completely by the object. In passive detection, the magnitude of stimulus can be increased as needed by altering the size and speed of the object. In contrast, the signal corresponding to the active identification is generally weaker since the flow is generated by the motion of the sensing body, which additionally has the issue of self-generated noise due to the motion. For these reasons, the initial research in object detection covered in this thesis will focus on passive object detection.

\subsubsection{The Lateral Line and Vortices}

Vortices are long-lived flow structures that generate regular pressure distributions and are therefore a likely target for interaction with the fish lateral line. Vortices are generated in fluid flow in a number of ways, but the primary methods of biological relevance are via the motion of a fish's tail when generating thrust and in the interaction between a bluff body and a free stream (such as the flow in a river). In both of these scenarios, some fish species have learned to make use of their lateral line to take advantage of the vortices.

All fish generate persistent vortices in their wake when they swim with a caudal fin. This fact has allowed predators to track and capture prey even when they are unable to see the prey, by following the wake left in the fluid. This behavior was shown conclusively by Pohlmann et al. in catfish $[64,63]$ which could track prey wakes up to ten seconds after they passed. Additionally, it is clear based on those experiments that the lateral line 
is a critical component in the behavior. Without a functional lateral line, the method of prey capture shifted from predominantly wake following (55\%) to almost entirely chance encounter (6\% wake following). This research demonstrates the lateral line's use in wake following, which is most likely associated with vortices in the wake as they are an important persistent component of the wake. However, it does not demonstrate that the lateral line is used to identify individual vortices.

Certain fish, such as trout, have been observed to adopt unique gaits when swimming behind cylinders in steady flow in order to take advantage of the alternating vortices shedding from the cylinder in order to reduce the energy needed to maintain position in the flow $[46,45]$. This motion by the fish requires tuning to the position of the vortices with respect to the fish body [44]. While the behavior is complex and depends on more than just the lateral line, the removal of the lateral line causes a clear alteration in the motion of the fish when trying take advantage of the wake [43]. In addition, it has been found that the vortex shedding frequency is encoded in the nerve fibers connected to the lateral line [10], indicating that at least some high level information about vortices is being captured. The response of fish to the wake of a cylinder is a clearer indication of vortex tracking via the lateral line, since the relative position of the vortices to the fish body is of importance for minimizing the energy expenditure of swimming. However, as of yet there is no confirmation that the fish lateral line is used to extract the position of individual vortices.

Similar to the conclusions with the lateral line and object identification, there are many factors that indicate that a lateral-line-like sensor can estimate detailed real-time information about nearby vortices. This is based on the complementary nature of vortices with respect to the measured signals and the fish behaviors that appear to utilize vortices and in some cases the particular location of vortices. However, in order to use a lateral-line-like sensor to track vortices, questions about the limits of the information available will need to be addressed as well as the actual implementation. So far, the knowledge of the biological lateral line is unable to contribute to these goals.

It is interesting to note that vortices play a role in tracking prey for other species that do not have a lateral line, specifically harbor seals. Using their specially shaped whiskers, which may dampen self-generated vibrations [31], these seals have been shown able to 
track the path of a small mechanical submarine while blindfolded, sevearl seconds after the submarine had gone [22]. The only method of achieving this feat requires tracking the path via the wake, which is most significantly composed of vortices. While this does not have a direct impact on the implementation of a lateral-line-like sensor array, it further reinforces the importance and the detectability of vortices under similar situations.

\subsection{Conclusions}

The fish lateral line is a versatile sensory organ that enables some surprising behaviors in fish. This thesis is focused on the abilities of the lateral line to identify rigid bodies and vortices, which have been associated with the trunk canal neuromasts of the lateral line. This canal section of the lateral line responds to pressure gradients on the surface of the fish along a horizontal line. Based on these observations, the sensory system chosen to implement similar abilities on man-made objects is a linear array of pressure sensors. While a two dimensional array may improve the ability to estimate three dimensional stimuli, the lateral line model provides an indication of a sufficient yet simple sensory arrangement for the tasks of interest.

In examining the ability of fish to identify rigid bodies and vortices, there is plenty of circumstantial evidence that suggests a lateral-line-like sensor would be able to accomplish both tasks. However, there remain significant unanswered questions, particularly with respect to the level of detail of information available via the lateral line. These questions need to be answered experimentally from the engineering perspective, and this is the principal goal of this thesis. 


\section{Chapter 3}

\section{Tracking a Cylinder in Motion}

This chapter focuses on the question of whether it is possible to identify in real time the position and size of a moving cylinder based solely on an array of pressure sensors. Since the cylinder is moving relative to the fluid, it generates a wake which is significant factor in the ability to track a cylinder. While, Chapter 5 of this thesis separately addresses the question of tracking the individual vortices generated in the wake of a cylinder, in the present chapter, the wake will be dealt with more abstractly. Specifically it will be considered as a part of the whole pressure field generated by a moving cylinder. On a separate note, although the focus is on a moving cylinder coupled with stationary sensors, the results will be applicable to situations such as a stationary cylinder in a current.

The question of the position and size of a cylinder deserves separate consideration from the question of identifying the shape of a cylinder. The position and size can be considered the most fundamental elements of a moving cylinder. The position is necessary if the shape of the cylinder is going to be estimated in real time. Similarly the size of the cylinder crosssection can be considered the lowest order information about the shape. While information about the shape in the pressure field will decay with distance in potential flow, the size of the cylinder is one of the slowest decaying signals. Given the relevance of the position and size, it is important to characterize the conditions under which it is possible to accurately estimate them. This is a precursor to investigating the question of identifying the shape of a cylinder.

Although the inspiration for the question of whether an array of pressure measurements 
captures sufficient information to track a cylinder in real time originates with the lateral line organ in fish, there is little information available on whether fish are able to extract this specific information (see Chapter 2). From the evolutionary perspective, there is reason to believe that such information would be relevant to a fish. In particular, fish that sit and wait on a lake floor for prey to pass above, such as the mottled sculpin [16], may benefit from the ability to locate nearby fish and classify them as predator or prey depending on the size. However, whether this occurs is only speculation at this point. As with the rest of the thesis, the pressure sensor array considered in this chapter is restricted to a linear and horizontally aligned arrangement, much like the trunk lateral line in fish. In this sense, results of the present investigation provide an indication of whether it would be possible for a fish to use its trunk lateral line in such a manner.

\subsection{Strategy and Motivation}

A round cylinder is the most appropriate starting point for approaching the question of tracking a cylinder with a general cross section. As seen in the parameterization by Bouffanais et al [6], the shape of a cylinder can be easily expressed as a series of perturbations of increasing complexity based on a conformal map. As the complexity increases, the order of the pole needed to represent the perturbation in potential flow also increases, resulting in a faster decay of the pressure generated by the perturbation. The lowest-order shape, a circle, therefore has the largest applicability to other shapes, especially when the cylinder is a some distance from the sensors. Though the presence of the wake implies that the lessons from potential flow cannot be directly applied, the relationship between small complicated perturbations in a shape and quickly decaying contributions to the near field pressure will still hold.

There are several stages in evaluating whether a linear array of pressure sensors can be used to estimate the position and size of a moving cylinder in real time. These are: choosing an adequate model for the cylinder motion and pressure measurements; choosing, adapting, and testing an estimation algorithm; and, finally, evaluating the performance of the cylinder state estimation. While there are many different possibilities for modeling the pressure gen- 
erated by a moving cylinder, the focus here is on a constant analytic model in the interest of reduced computation for real-time estimation. The choice of estimation algorithm depends on the model and the measurement noise, but many well-known inference techniques are available given the format of the problem. Once a successful estimation technique has been identified, it is of interest to determine whether the estimation is equally accurate under all conditions, or whether certain types of moving cylinders are better estimated.

In order to investigate the question of tracking a moving cylinder in an open environment in a realistic setting, it is necessary to apply estimation techniques to experimentally acquired data. In particular, the problem must also be considered from an experimental point of view in order to account for the effect of realistic non-electrical noise. A priori, it is unknown what noise sources are the most significant from the point of view of tracking a cylinder. For instance, the unwanted signals may be generated from persistent eddies in the experimental tank or from surface oscillations. By experimentally characterizing the noise sources and testing the estimation algorithms on physically generated data, it will be possible to develop a robust implementation.

\subsection{Model Evaluation and Selection}

In order to track the position and size of a circular cylinder, two components are necessary: a model of the physical situation and an algorithm for estimation. These two parts are distinct, and while the estimation technique depends on the specific characteristics of the measurements and experiment, such as the noise, the model does not. The model represents the forward problem which turns an initial set of conditions such as the initial location, speed, and size of the cylinder, and predicts the entire subsequent set of measurements. The inverse problem, going from the measurements to the current state of the cylinder, is dealt with through the estimation algorithm. In this chapter, the scenario to be modeled is that of a circular cylinder undergoing a steady translation at a reasonably high Reynolds number near a wall on which the sensors will be mounted. This is an idealization of the scenario in which an object passes near the hull of a ship. 


\subsubsection{General Model Form and State Transition}

Given some reasonable initial assumptions and the proper choice of state variables, the overall problem is in the form of a hidden Markov model (HMM). A hidden Markov model structure (Figure 3-1) is of importance because it opens up the possibility of using powerful estimation techniques, such as the Kalman filter. In a HMM there is a sequence of states that are invisible to the observer and of corresponding visible measurements, all of which are random variables. The lines in Figure 3-1 denote dependence between random variables. Thus in an HMM, the state at some point $n$ in the sequence only depends on the state that immediately preceded it $(n-1)$ and is completely independent of all earlier states if conditioned on the state at $n-1$. In addition, the measurement at a certain point in the sequence, or time, only depends on the state at the same time. Thus the measurement $m_{n}$ conditioned on all the states $q_{n} \ldots q_{0}$ is independent of the states $q_{n-1} \ldots q_{0}$.

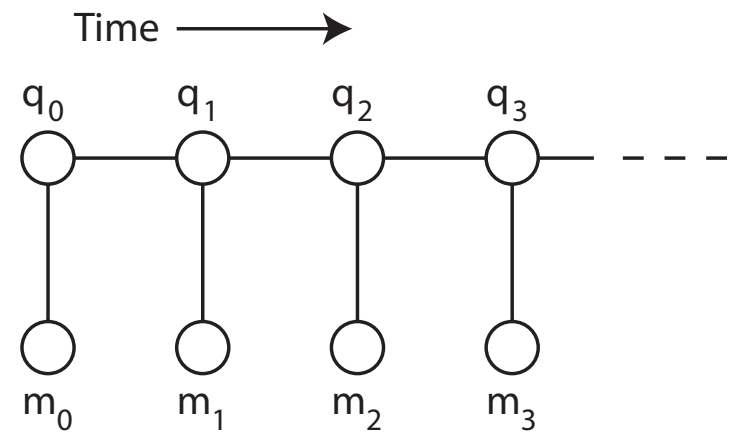

Figure 3-1: Hidden Markov model undirected graph. The diagram describes the relation between a series of random variables with edges denoting direct dependence between the random variables.

The aim of this chapter is to track the position and size of a moving cylinder, based on pressure measurements. Therefore, the position ( $x$ and $y$ ) and radius $r$ are natural state variables. Throughout this thesis, the $x$ direction will refer to the direction parallel to the linear sensor array, and the $y$ direction will be perpendicular with zero at the level of the array. Similarly to the state vector, the pressure measured at each point at the array naturally forms the measurement vector. In order to fit into the HMM structure however, the state needs to include the velocity of the cylinder. Thus the state transition can be written as only 
a function of the previous state:

$$
q_{n}=\left[\begin{array}{c}
x_{n} \\
y_{n} \\
r_{n} \\
u_{n}
\end{array}\right]=F\left(q_{n-1}\right)=\left[\begin{array}{c}
x_{n-1}+u_{n-1} d t \\
y_{n-1} \\
r_{n-1} \\
u_{n-1}
\end{array}\right]+\left[\begin{array}{c}
\eta_{x, n} \\
\eta_{y, n} \\
\eta_{r, n} \\
\eta_{u, n}
\end{array}\right]
$$

where $x$ and $y$ are the coordinates of the position of the center of the cylinder, $r$ refers to the radius of the cylinder, and $u$ is the velocity of the cylinder (assumed to be along the $x$ direction only for simplicity). In addition, $\eta_{x}, \eta_{y}, \eta_{r}, \eta_{u}$ are random variables that capture the noise and unmodeled effects in the transition.

This expression of the state transition assumes that there are no memory effects that would cause the position, size, and speed of the cylinder at one time instant to be affected from a previous state in addition to the immediate antecedent. This is a minor assumption, given that the cylinder is considered to be externally driven and following a preset path. However, according to the HMM structure, there should also be no memory effects that cause the measurement at some time instant to be affected by anything other than the current state of the cylinder. This is a more substantial assumption. In particular, vortices shed in the wake of the cylinder will result in a persistent signal that is dissociated with the state of cylinder at later times. In formulating the problem as a hidden Markov model, we assume that a vortex will infrequently be shed near the sensors.

\subsubsection{Measurement Models}

The remaining component of the model to be specified relates the state at any given moment to the corresponding measurements. In order to achieve real time tracking of the cylinder state, different analytical models were considered in order to identify one which captured the dominant characteristics of the pressure response due to a moving cylinder. Any analytical model will, at best, be an approximation to the real situation since it is unable to account for the unsteady aspects of the fluid motion. An alternative would be to run an approximate viscous computational fluid dynamics model, which would be substantially 
more computationally expensive.

The standard analytical model for a cylinder in a steady uniform flow in an inviscid, irrotational fluid is created with a dipole, where the complex potential is $\Phi(z)=$ $u\left(z+\frac{a^{2}}{z-z_{0}}\right)$. In this equation, $z=x+i y$ and $z_{0}$ refers to the complex location of the center of the cylinder. The radius of the modeled cylinder is denoted by $a$. The complex potential $\Phi$ is a combination of the velocity potential and stream function, $\Phi=\phi+I \psi$, and the flow velocity can be obtained by: $u-I v=\frac{\partial \Phi}{\partial z}$. The dipole model is severely inadequate for tracking a moving cylinder, due to its complete lack of a wake. This not only affects the pressure once the cylinder has passed a sensor, but changes the complete near-field pressure distribution including ahead of the cylinder, as demonstrated shortly.

Three models were considered which would capture aspects of the wake of the cylinder better than a dipole. The first model is an analytical potential flow model similar to the dipole model, in which a source is instead used as the singularity for forming the structure. The corresponding complex potential is:

$$
\Phi(z)=u\left(z+a \log \left(z-z_{0}\right)\right)
$$

As previously, $z_{0}$ refers to the center of the cylinder. Also $a$ is the radius of the cylinder, which is taken to be the distance between the stagnation point and the center. This model is commonly referred to as a Rankine half-body [60]. The second model under consideration is a hybrid of the rankine half body with a dipole. The combination of the two allows the width of the wake to be altered independently from the radius of the cylinder, unlike in the case of the Rankine half-body. The corresponding complex potential for this hybrid model is

$$
\Phi(z)=u\left(z+\frac{\beta^{2}}{z-z_{0}}+\frac{a^{2}-\beta^{2}}{a} \log \left(z-z_{0}\right)\right)
$$

Here, $a$ is still the radius of the cylinder, and $\beta$ is a weighting factor that ranges from 0 to $a$ corresponding to the range between just a source and just a dipole. The final model is based on a slightly different approach, in which a shadow of the cylinder was manually extended downstream from the furthest lateral points on the cylinder in order to simulate a thin wake. The flow was evaluated around this extended cylinder using a panel method. A 
depiction of the streamlines passing through the stagnation point for each model is shown in Figure 3-2 for situations both near and far from a wall.

These potential models were used to obtain the near field pressure via the unsteady Bernoulli equation [60]:

$$
P(z)+\frac{1}{2} \rho\left|\frac{\partial \Phi(z)}{\partial z}\right|^{2}+\rho \frac{\partial \phi(x, y)}{\partial t}=P_{\infty}+\frac{1}{2} \rho u_{\infty}^{2}
$$

Note that the derivative in time is with respect to the velocity potential only (the real component of $\Phi)$, and is therefore always real and a function of $x$ and $y$ separately rather than of $z=x+i y$ alone. ${ }^{1}$ Also, this unsteady form of the Bernoulli equation applies to every instant in time separately, but considering the pressure at a spatially distant points constant enables the comparison across time.

\subsubsection{Measurement Model Frame Equivalence}

Although the problem scenario is that of a cylinder steadily translating through still water next to a flat plate, this is equivalent to the scenario in which the cylinder is stationary under a steady uniform flow next to a flat plate with the position of the sensors translating on the plate. This equivalence holds due to the assumption that the flat plate extends infinitely, and thus there is no difference in the potential model except the change in location. It is similarly valid if there is no sensing body included in the model. The equivalence can be seen explicitly by considering the Bernoulli equation applied to the two scenarios with a general complex potential model. Let $z_{0}$ refer to a fixed position for the cylinder corresponding to the case with the moving sensor frame of reference, and $\tilde{z}_{n}=z_{0}-u t_{n}$ be the position of the moving cylinder at time-step $n$ in the physical scenario. For the purpose of demonstrating the equivalency, let both the velocity and perpendicular distance from the sensors be constant: $u_{n}=u$ and $\tilde{y}_{n}=y_{0}$. Correspondingly, consider a general complex potential $\tilde{\Phi}\left(z-\tilde{z}_{n}\right)$ for the moving cylinder in still water, which is related to the complex potential of the stationary cylinder in a steady flow by $\Phi\left(z-z_{0}\right)=\tilde{\Phi}\left(z-z_{0}\right)+u z$. The

\footnotetext{
${ }^{1}$ The distinction made here is between, for instance, $x e^{x+i y}$ and $(x+i y) e^{x+i y}$. The latter can be expressed in terms of the $z=x+i y$ variable alone, yielding $z e^{z}$, whereas the former cannot.
} 
argument of the complex potential is written explicitly as $z-z_{0}$ in order to denote the relationship between the point of evaluation, $z$, and the center of the cylinder $z_{0}$. Note that all the complex potentials discussed so far have this form (except the shadow wake generated by the panel method, which is similar but without all the singularities centered at $z_{0}$ ). In the case of the stationary cylinder, the Bernoulli equation reduces as:

$$
\begin{aligned}
& P(z)+\frac{1}{2} \rho\left|\frac{\partial \Phi\left(z-z_{0}\right)}{\partial z}\right|^{2}+\rho \frac{\partial \phi\left(x-x_{0}, y-y_{0}\right)}{\partial t}=P_{\infty}+\frac{1}{2} \rho u_{\infty}^{2} \\
& P(z)+\frac{1}{2} \rho\left|\frac{\partial\left(\tilde{\Phi}\left(z-z_{0}\right)+u z\right)}{\partial z}\right|^{2}=P_{\infty}+\frac{1}{2} \rho u^{2} \\
& P(z)-P_{\infty}=-\frac{1}{2} \rho\left|\frac{\partial \tilde{\Phi}\left(z-z_{0}\right)}{\partial z}+u\right|^{2}+\frac{1}{2} \rho u^{2} \\
& P(z)-P_{\infty}=-\frac{1}{2} \rho\left(\left|\frac{\partial \tilde{\Phi}\left(z-z_{0}\right)}{\partial z}\right|^{2}+2 u \Re\left[\frac{\partial \tilde{\Phi}\left(z-z_{0}\right)}{\partial z}\right]+u^{2}\right)+\frac{1}{2} \rho u^{2} \\
& P(z)-P_{\infty}=-\frac{1}{2} \rho\left(\left|\frac{\partial \tilde{\Phi}\left(z-z_{0}\right)}{\partial z}\right|^{2}+2 u \frac{\partial \tilde{\phi}\left(x-x_{0}, y-y_{0}\right)}{\partial x}\right)
\end{aligned}
$$

Considering the case of a moving cylinder passing the same point as the stationary cylinder, without external flow:

$$
\begin{aligned}
& P(z)+\frac{1}{2} \rho\left|\frac{\partial \tilde{\Phi}\left(z-\tilde{z}_{n}\right)}{\partial z}\right|^{2}+\rho \frac{\partial \tilde{\phi}\left(x-\tilde{x}_{n}, y-y_{0}\right)}{\partial t}=P_{\infty}+\frac{1}{2} \rho u_{\infty}^{2} \\
& P(z)+\frac{1}{2} \rho\left|\frac{\partial \tilde{\Phi}\left(z-\tilde{z}_{n}\right)}{\partial z}\right|^{2}+\rho \frac{\partial \tilde{\phi}\left(x-\tilde{x}_{n}, y-\tilde{y}_{n}\right)}{\partial \tilde{x}_{n}} \frac{\partial \tilde{x}_{n}}{\partial t}=P_{\infty}+\frac{1}{2} \rho 0^{2} \\
& P(z)+\frac{1}{2} \rho\left|\frac{\partial \tilde{\Phi}\left(z-\tilde{z}_{n}\right)}{\partial z}\right|^{2}+\rho \frac{\partial \tilde{\phi}\left(x-\tilde{x}_{n}, y-y_{0}\right)}{\partial x} u=P_{\infty} \\
& P(z)-P_{\infty}=-\frac{1}{2} \rho\left|\frac{\partial \tilde{\Phi}\left(z-\tilde{z}_{n}\right)}{\partial z}\right|^{2}-\rho \frac{\partial \tilde{\phi}\left(x-\tilde{x}_{n}, y-y_{0}\right)}{\partial x} u \\
& P(z)-P_{\infty}=-\frac{1}{2} \rho\left(\left|\frac{\partial \tilde{\Phi}\left(z-\tilde{z}_{n}\right)}{\partial z}\right|^{2}+2 u \frac{\partial \tilde{\phi}\left(x-\tilde{x}_{n}, y-y_{0}\right)}{\partial x}\right)
\end{aligned}
$$

Since $\tilde{z}_{n}(t=0)=z_{0}$, both frames of reference give the same results. For compactness, the stationary cylinder frame is preferred since there is no time derivative in the corresponding in the Bernoulli equation. 


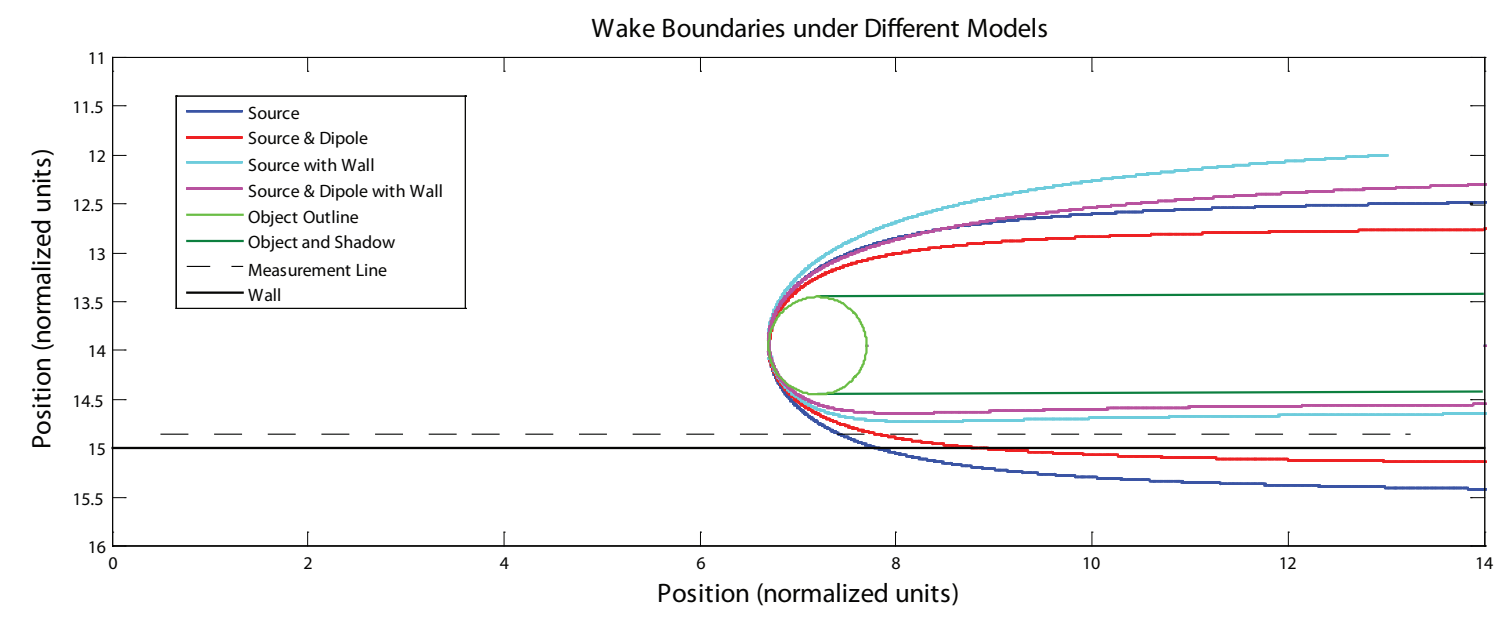

Figure 3-2: Model wake boundaries. The wakes for each model are superposed around the outline of the actual cylinder for comparison. The wakes are depicted near a wall and in open water with the solid black line denoting the location of the wall.

In order evaluate the accuracy of the models, an unsteady viscous simulation was computed to obtain approximate solutions to the flow about a cylinder. The software used for these simulations was a custom boundary domain integral method (BDIM) solver developed by Gabriel Weymouth (personal communications). The layout of the two dimensional simulation consisted of a round disk positioned in a square volume with no flux boundary conditions on the top and bottom walls. Using this computational fluid dynamics (CFD) software, the pressure along a line in the flow was measured over a number of vortex shedding periods. The measurement line was positioned both in the open flow and adjacent to the wall for different simulations. In these simulations, the cylinder was stationary under a constant free stream. However, since the wall is flat, parallel to the direction of fluid motion, and infinitely long, this frame of reference is equivalent to that of a cylinder moving parallel to a flat wall at constant speed.

The simulated pressure measured on the linear array has some general characteristics that are consistent in both the case near a wall (Fig 3-4) and away from one (Fig 3-3). Generally, the pressure has a steady exponential rise in front of the cylinder, representing the effect of the bow pressure. Subsequently there is a smooth maximum in the pressure followed by a steep transition The pressure simulated along the array also has substantial variation in the peak negative pressure and recovery with respect to the far field. Compar- 
ing the results between a cylinder near a wall and a cylinder away from one, the former generates a substantially larger amplitude pressure signal. The presence of a wall also substantially decreases the variation in pressure due to the vortex shedding. This affect is most evident in the region upstream of the cylinder. It is also noticeable however downstream of the cylinder, where the variation in pressure due to the wake is much more substantial in the absence of a wall.

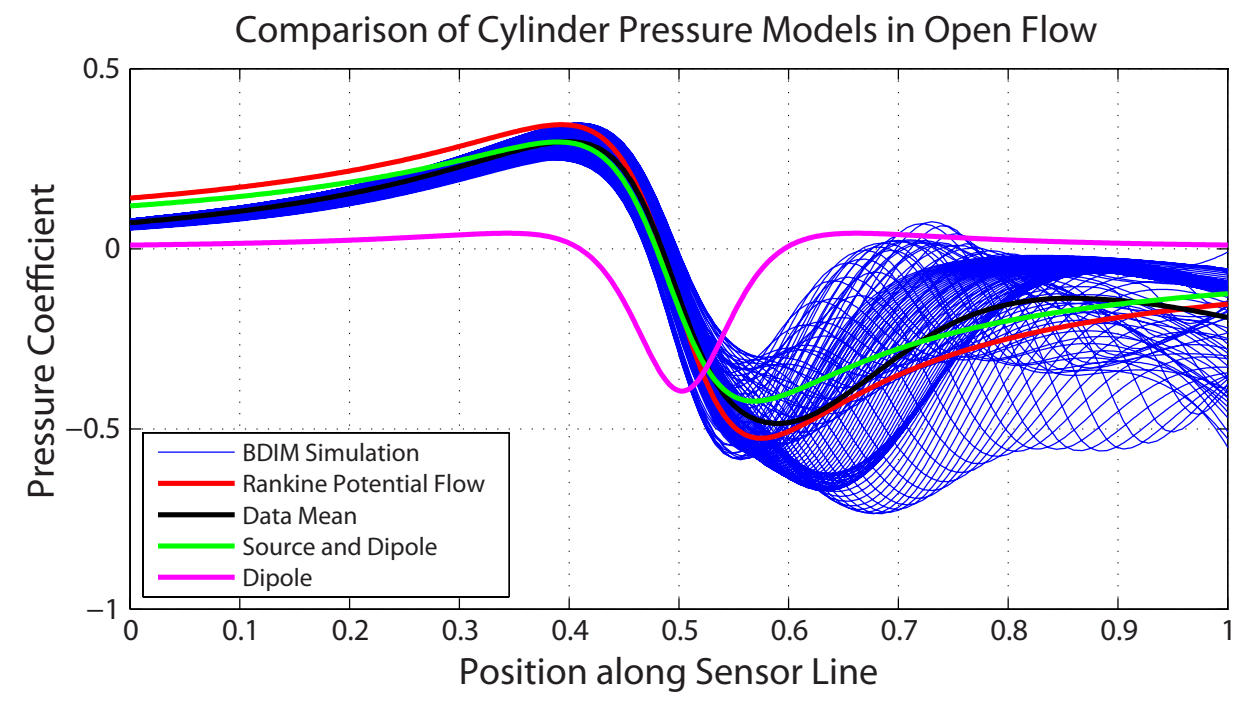

Figure 3-3: Pressure comparison between models and CFD simulation in open water. The simulated CFD pressure over a ghost sensor array is displayed at regular intervals over multiple shedding periods in blue. The mean of these simulated pressure traces (in black) is compared to the results from the analytical models.

The dipole model of a cylinder in a flow is also included in the comparison against the simulated pressure (Figure 3-3) in order to clarify how poorly it models a cylinder when the wake is present. To start, the amplitudes mismatch substantially, particularly in the maximum pressure. In addition, the entire pressure response appears shifted so that the peak minimum pressure roughly corresponds to the zero crossing point in the simulated pressure response. The symmetry of the dipole pressure model is expected since it does not model a wake, but it is clear from this comparison to the simulated data that even the region nominally ahead of the wake does not correspond to the dipole model.

In contrast to the dipole model, both the source model (Rankine half-body) and the source-dipole model capture the general behavior of the simulated pressure signal. In these 
comparisons, the parameter determining the width of the wake was set to the value minimizing the least-squares difference with respect to the mean simulated pressure trace away from a wall. Both the source and the tuned source and dipole model approximately match the mean simulated data's amplitude in the pressure response and are closely aligned in position at the point were the pressure transitions from positive to negative. However, both models share the same faults at either extreme of the pressure response. Upstream of the cylinder, the decay in pressure is dominated by the source singularity and decays too slowly with respect to the simulated results. As expected, downstream of the cylinder, the two models only roughly match the average decay from the simulated data. This is unsurprising, since there is a large amount of variation in the pressure corresponding to the wake region which cannot be captured by a single steady analytical model.

The primary difference between the source and the source and dipole models is in the amplitude of the pressure response near the transition region. At the positive pressure peak, the model with the dipole thinning the wake matches the mean simulated results very well, while the rankine model overestimates the maximum pressure. For the peak minimum pressure, both models differ from the mean simulated response by about an equal amount, but with the source and dipole model making an overestimate while the rankine model underestimates. Although there are differences between the pressure predicted by the two models, they are small with respect to the variation in pressure that occurs within one vortex shedding period.

The results from the panel method model in which only the shadow of the cylinder was projected to simulate a wake resulted in pressure whose amplitude fell below the projected values by a substantial margin, remaining at less than half the amplitude of the simulated results. This clearly indicates that the wake in this model is too small. Since both the Rankine and the source-dipole models predict a pressure distribution that is much closer to the CFD results, the directly specified panel method wake will not be considered further.

In the case of a simulated cylinder away from a wall, both the Rankine and the source dipole mixture capture the appropriate transition behavior in pressure. Near a wall, the situation changes only slightly (Figure 3-4). The effect of the wall increases the amplitude pressure measured in the sensor array. Using the same wake thickness as for the no-wall 
simulations, the source and dipole model still captures the maximum pressure very well and now is much closer to the minimum peak pressure. The model with the source alone over estimates the magnitude of the pressure throughout the transition area. Beyond these differences, the agreement with the simulated pressure results is no different than in the nowall case. When a wall is present, the source-dipole model predicts the correct amplitude in pressure on the sensor array, while the Rankine model consistently overestimates it.

One important note is that when a wall is present, the Rankine model will result in pressure that nearly overlaps the source-dipole model if the radius of the Rankine model cylinder is reduced ten percent. This implies that there are few significant differences between the overall shape of the pressure response from the two models, but there is a difference in the modeling of the size of the cylinder. If the Rankine half-body model is used for solving the inverse problem, it should be expected that the size of the cylinder will be under-estimated.

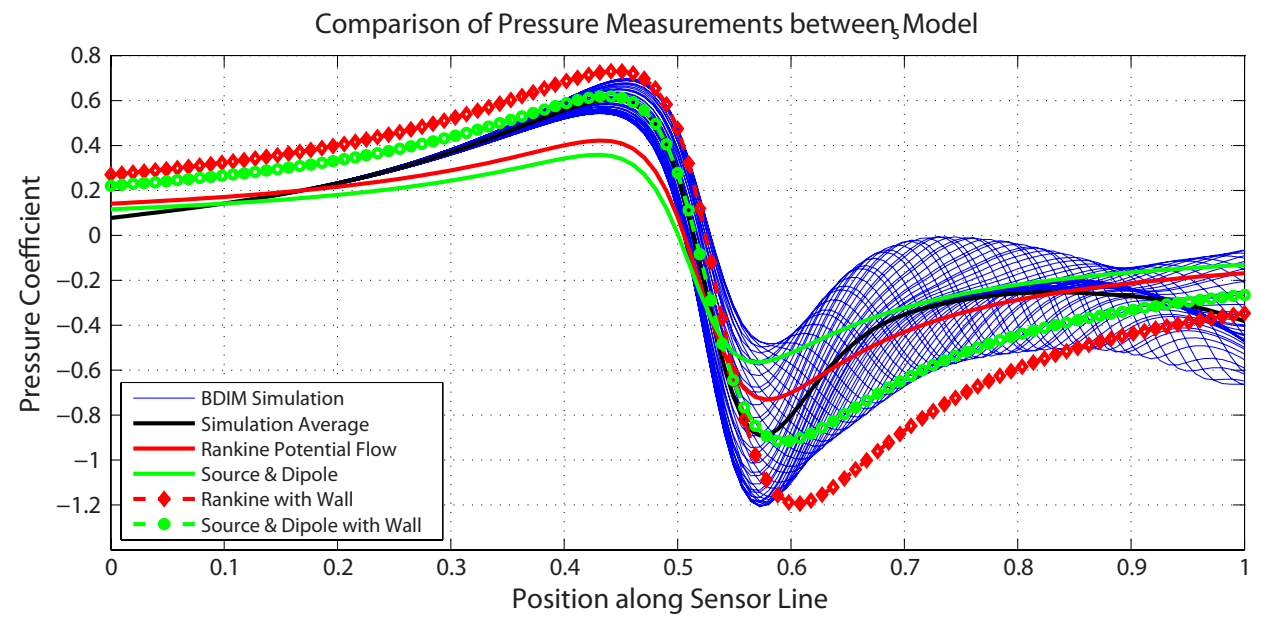

Figure 3-4: Pressure comparison between models and CFD simulation near a wall. The CFD results are displayed in blue at various intervals of the vortex shedding period. This graph corresponds to the layout described in Figure 3-2. The results of the Rankine halfbody and the source-dipole hybrid model are plotted both with and without the wall present.

The conclusions from the CFD simulations of a cylinder in a steady flow indicate that an analytical model based on a source and dipole will predict the pressure on a linear sensor array well in the vicinity of the cylinder, but with less accuracy and some systematic bias both up and downstream of the cylinder. The Rankine model behaves similarly, with 
slightly worse performance in the open water case compared to the source-dipole model, and an underestimate of the cylinder size when near the wall. However, the purpose of examining these models is to obtain a basis for the tracking of a cylinder in real time in a real environment. For that purpose, the Rankine model is better suited than the sourcedipole model if there is a wall near. Due to the image of the cylinder needed to represent the wall, the stagnation point used to define the size of the cylinder migrates towards the wall, and modeling a cylinder of a specified radius requires further costly calculations. In comparison, the Rankine model has a similar migration of the stagnation point, but the model can still be analytically expressed as a function of the cylinder diameter. The cost of using the Rankine model would be predicted to be a slight underestimate of the radius of the cylinder, but since there is no difference between the responses when using a smaller radius, it should not affect the ability to track a cylinder.

In order to evaluate ability to track a cylinder based on a Rankine model, data was collected from a series of experiments representing the scenario of interest. The fundamental characteristic of the experiment was to pass a circular cylinder moving at constant velocity past an array of pressure sensors with a precise specified distance, matching the scenario used for the model.

\subsection{Experimental Setup}

The experiments were conducted in the CENSAM Singapore experimental tank. The CENSAM tank is $3.6 \mathrm{~m}$ long by $1.2 \mathrm{~m}$ wide and approximately $1 \mathrm{~m}$ deep. Two separate components are involved in the experiment: a method for moving a cylinder along a precise path and a sensing platform that reproduces the ability of a lateral-line like sensor array embedded in an infinite wall.

\subsubsection{Cylinder Motion}

On the top of the tank, a two-dimensional positioning system allows accurate position and velocity specification over the water. The positioning system is composed of an "H" configuration of belt-driven linear actuators (Parker LCB-40). Two 3.6 meter long actuators 
are driven in concert by a single motor (Parker SMH servo motor type 60), and the cross piece $(1.2$ meters $)$ is independently actuated by a duplicate motor. This system allows placement with sub-millimeter accuracy anywhere over the tank, which is necessary given the sensitivity of the experiments to the distance between the sensors and the object.

The cylinder in the experiments is a hollow, $1.3 \mathrm{~m}$ long, $32 \mathrm{~mm}$ diameter cylinder with a round cross section. It is mounted rigidly in a vertical orientation to the crosspiece of the gantry system. In order to locate the position of the cylinder parallel to the sensors (x direction in Figure 3-5), a light switch was aligned to trigger when the cylinder was centered in front of the first sensor. To specify the position in the y direction, perpendicular to the sensor array, the cylinder moved using the gantry system and referenced against the point of contact between the cylinder and the sensor array.

The motion of the cylinder was chosen to minimize extraneous stimuli and noise sources. The basic motion consisted of a velocity ramp on either end of a constant specified velocity for the portion of the motion in front of the sensors. The acceleration of the initial ramp was reduced as much as possible such that the ramp was completed before any sign of the stimuli could be measured by the sensors. This acceleration cap was based on the most sensitive of the experiments (at highest velocity and closest distance) and maintained for all the other experiments. The acceleration cap was necessary to avoid or at least minimize the excitation of the cylinder vibration that was observed during sudden starts. The deceleration was symmetric to the acceleration.

\subsubsection{Sensing Platform}

The sensors involved in these experiments were Honeywell 19C015PG4K gauge pressure sensors. These sensors have a range of 15 psi (approx. $104 \mathrm{kPa}$ or $10 \mathrm{~m}$ of standing water) and an output of 0-100 mV. Seven sensors were equally spaced in a horizontal linear array with 0.75 inch $(0.019 \mathrm{~m})$ separation, centered on a flat square acrylic plate with a side length of 16 inches $(.4 \mathrm{~m})$ and 0.5 inch $(0.013 \mathrm{~m})$ thickness. The sensors were directly mounted onto the plate. Each sensor was connected to the flow by a small 1-2 $\mathrm{mm}$ port (see Figure 3-5) in order to minimize any pressure averaging effect. In order to maximize 

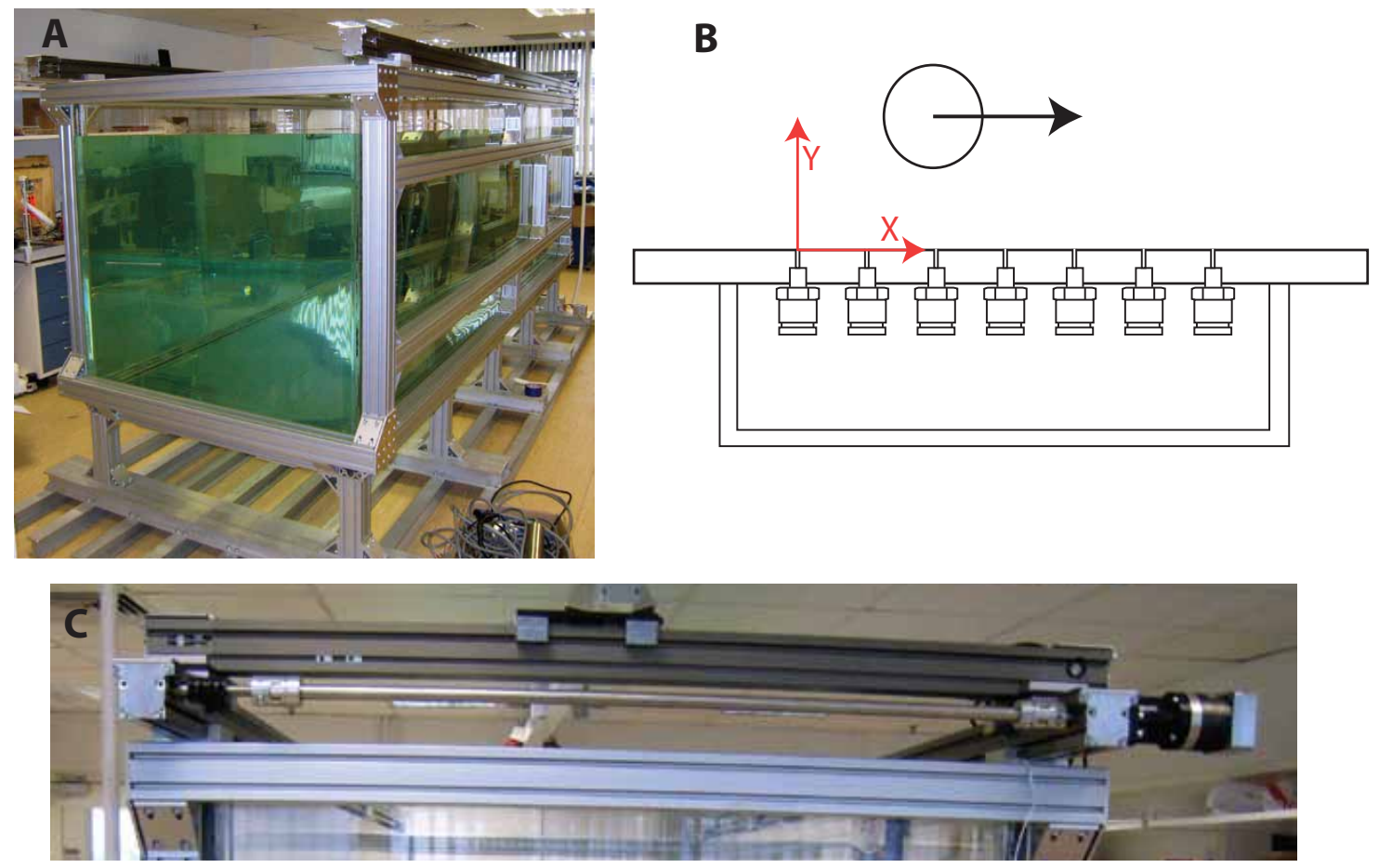

Figure 3-5: Cylinder tracking experimental setup. A: The tank in which the experiments were performed. B: Top-down diagram of the linear sensor array setup. The coordinate system for the experiments and models is shown, with the origin at the first sensor and the $\mathrm{X}$ direction parallel to the linear array. C: Detail of the positioning system from the rear. The crosspiece is visible with the stage that forms the point of connection for the cylinder.

sensitivity and minimize any nonlinearity due to an air-water interface navigating the port hole, the space between the sensors and external flow were completely flooded prior to the experiment, using a small syringe. The result is a single medium and a short direct path from the pressure at the location of interest on the plate and the corresponding sensor.

Behind the plate, the sensors were enclosed in a common compartment which served the purposes of providing a consistent reference pressure and dry space for amplification of the sensor signals. The signals from the pressure sensors were amplified immediately adjacent to the sensors themselves to offset the signal loss due to the long cables required, thus maintaining a high signal-to-noise ratio. The amplification was accomplished using AD620 instrumentation amplifiers set to amplify by a factor of 970 . Small capacitors $(0.1$ $\mu F$ ) were used to insulate the circuit from fluctuations in the current from the amplifiers. The full schematic for an individual sensor can be seen in Figure 3-6. The amplification of 


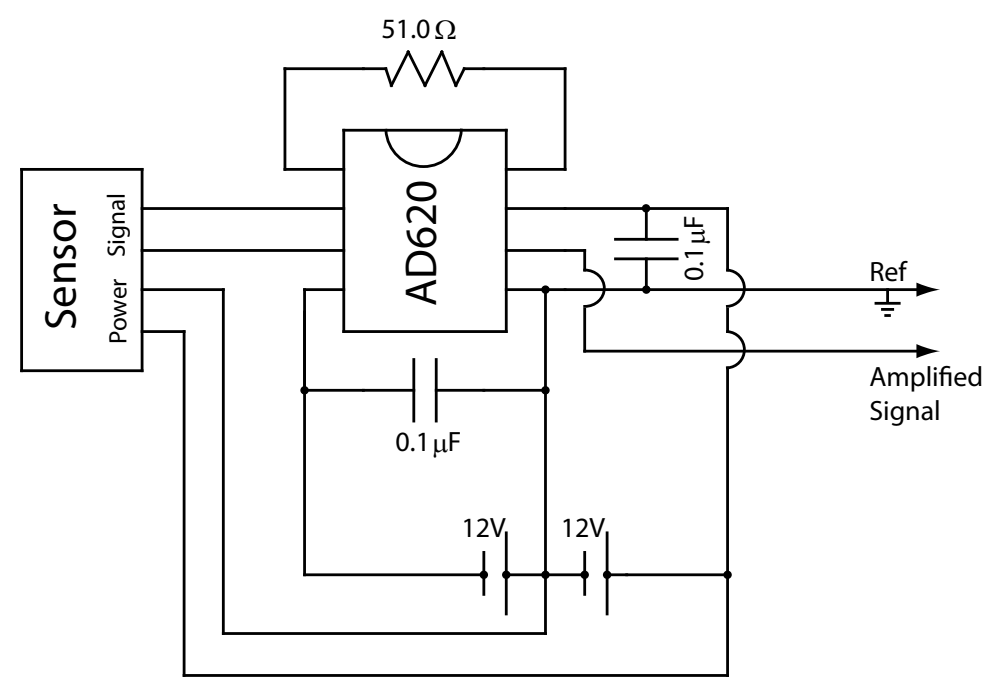

Figure 3-6: Amplification circuit diagram. This schematic describes the amplification circuitry module for a single pressure sensor. The arrows at the right of the diagram indicate the signal out to data acquisition. In implementation, all the sensors were powered and amplified by the same set of batteries.

the instrumentation amplifier is set by the resistor. In addition, capacitors isolate the rest of the circuit from possible fluctuations in the instrument amplifier chip. The amplified output was routed using a foil and mesh shielded cable to a National Instruments data aquisition system (NI USB-6210) powered directly from an unplugged laptop in order to minimize noise. The cable was kept as short as possible at about four meters, also for noise reasons. The power for the sensors and amplification circuit was provided by two $12 \mathrm{~V}$ batteries. The batteries were kept outside of the sensor enclosure and tank, connected by a shielded cable. This minimized the risk of leaks when the batteries were removed for charging.

Calibration of the sensors was accomplished by comparing the difference between the pressure at the operating depth and the surface $(40 \mathrm{~cm})$. The equation for static pressure: ${ }_{2}^{1} \rho g h=P-P_{o}$ is used to calculate the correct pressure difference based on the depth. Here, $\rho$ is the water density, taken to be $990 \mathrm{~kg} / \mathrm{m}^{3}, g$ is the gravitational acceleration $9.81 \mathrm{~m} / \mathrm{s}^{2}, h$ is the depth, $P$ is the pressure at depth, and $P_{o}$ is the pressure at the surface. This calibration was checked at the start of the experiment and again at the end, to check for any long term drift. 


\subsubsection{Test Matrix}

To quantify the dependence of the tracking accuracy on the motion parameters, a series of experiments were performed with the same cylinder and experimental setup. The first of the two variables of interest is the speed of the cylinder, which affects the strength of the pressure field and the Reynolds number. The wake characteristics and Reynolds number are closely tied. The second variable of interest is the minimum distance from the sensors in the path of the cylinder (in the $y$ direction according to Figure 3-5). The specific matrix of tests is described in Table 3.1 The velocity ranges from $125 \mathrm{~mm} / \mathrm{s}$ to $313 \mathrm{~mm} / \mathrm{s}$, corresponding to a range of Reynolds numbers between 4,000 and 10,000. At the upper end of signal strength, the range of the two variables was limited by the speed of the gantry system and the minimum distance for which there was complete confidence in avoiding collisions. At the other extreme of the testing matrix, at a velocity of $125 \mathrm{~mm} / \mathrm{s}$ and distance of $20 \mathrm{~mm}$, the measured signal is on the order of $10 \mathrm{~Pa}$ which is comparable to the unprocessed noise levels. Due to the variability of the wake, five runs were carried out for each element of the testing matrix.

\begin{tabular}{|c|c|c|c|c|c|}
\hline & $2 \mathrm{~mm}$ & $5 \mathrm{~mm}$ & $10 \mathrm{~mm}$ & $15 \mathrm{~mm}$ & $20 \mathrm{~mm}$ \\
\hline $0.31 \mathrm{~m} / \mathrm{s}$ & 5 Runs & & & & \\
\hline $0.25 \mathrm{~m} / \mathrm{s}$ & 5 Runs & 5 Runs & 5 Runs & 5 Runs & 5 Runs \\
\hline $0.19 \mathrm{~m} / \mathrm{s}$ & 5 Runs & 5 Runs & 5 Runs & 5 Runs & 5 Runs \\
\hline $0.13 \mathrm{~m} / \mathrm{s}$ & 5 Runs & 5 Runs & 5 Runs & 5 Runs & 5 Runs \\
\hline
\end{tabular}

Table 3.1: Experimental matrix for cylinder tracking. The range of velocities and distances was determined by the signal strength and by the abilities and noise of the experimental setup.

\subsubsection{Noise Analysis and Data Preparation}

The signals of interest in these experiments are of very small magnitude. In the case of a strong response they are on the order of $200 \mathrm{~Pa}$, but for weaker stimuli they are on the order of $20 \mathrm{~Pa}$ or less. As a result, managing noise and inteference from external sources was of extreme importance in the experiments. Many aspects of the experimental setup were chosen in order to minimize noise, as described in section 3.3, but others had to be managed 
in post-processing. The primary identified sources of noise in the measurements were the gantry motors, physical vibrations in the cylinder, and low frequency standing waves in the tank.

The sensors themselves have a base level of white Gaussian noise, with a standard deviation of $0.36 \mathrm{~Pa}$ after amplification (Figure 3-7). The frequency response of the amplified sensor is relatively flat, with peaks corresponding to $60 \mathrm{~Hz}$ and its higher multiples.
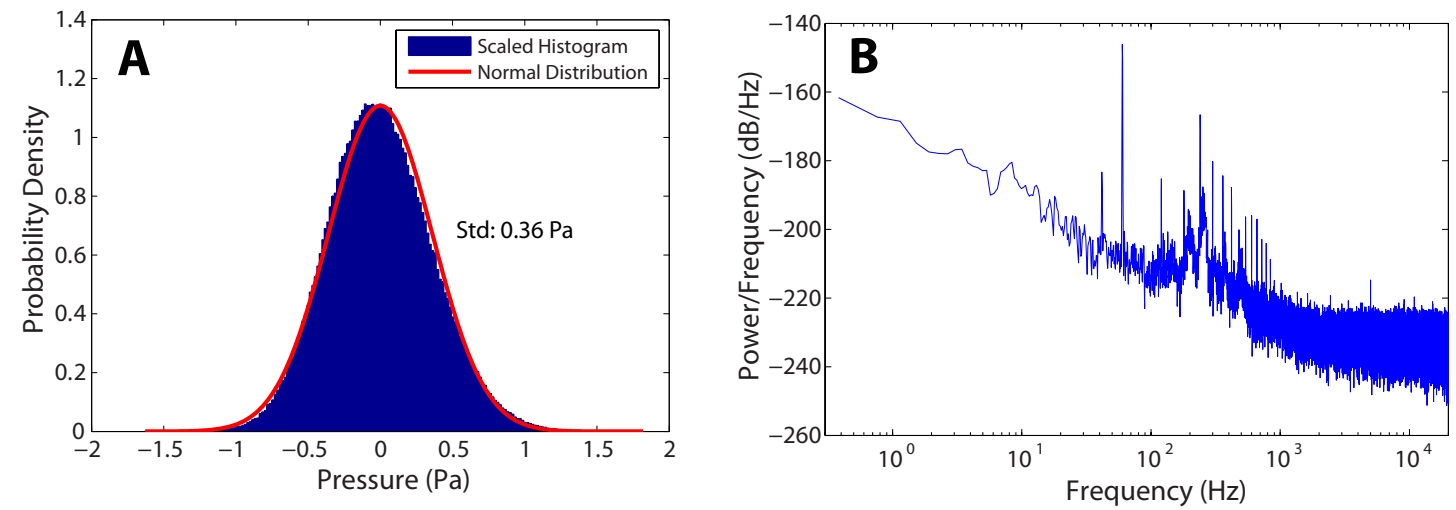

Figure 3-7: Sensor noise characterization. A: Histogram of noise over two seconds sampled at $30 \mathrm{kHz}$ with best-fit Gaussian distribution. B: Noise power spectrum

The motors provide a significant source of electrical interference, picked up through the cable between the data acquisition and the signal amplification. The interfering signal occurs as short high-frequency, large-magnitude bursts at intervals of 0.01 seconds. Aliasing of the high frequency component in the bursts causes substantial bias in the data. To avoid it therefore, we sample the sensors at $30 \mathrm{kHz}$, orders of magnitude faster than necessary for the experimental problem. This enables us to remove the high-frequency interference by post-processing, filtering the data prior to downsampling.

Vibrations of the cylinder as it moves past the sensors generate unwanted pressure signals and cause interference to the pressure sensors. The exact nature of the vibration is unclear. It is most likely due to slight stuttering in the motion of the gantry, but it is also possible that vortices shed from the cylinder play a role in stimulating it. In either case, the signals caused by the vibration of the cylinder have a single dominant frequency (approximately $13 \mathrm{~Hz}$ ) with an amplitude of up to roughly $15 \mathrm{~Pa}$ depending on the velocity. 

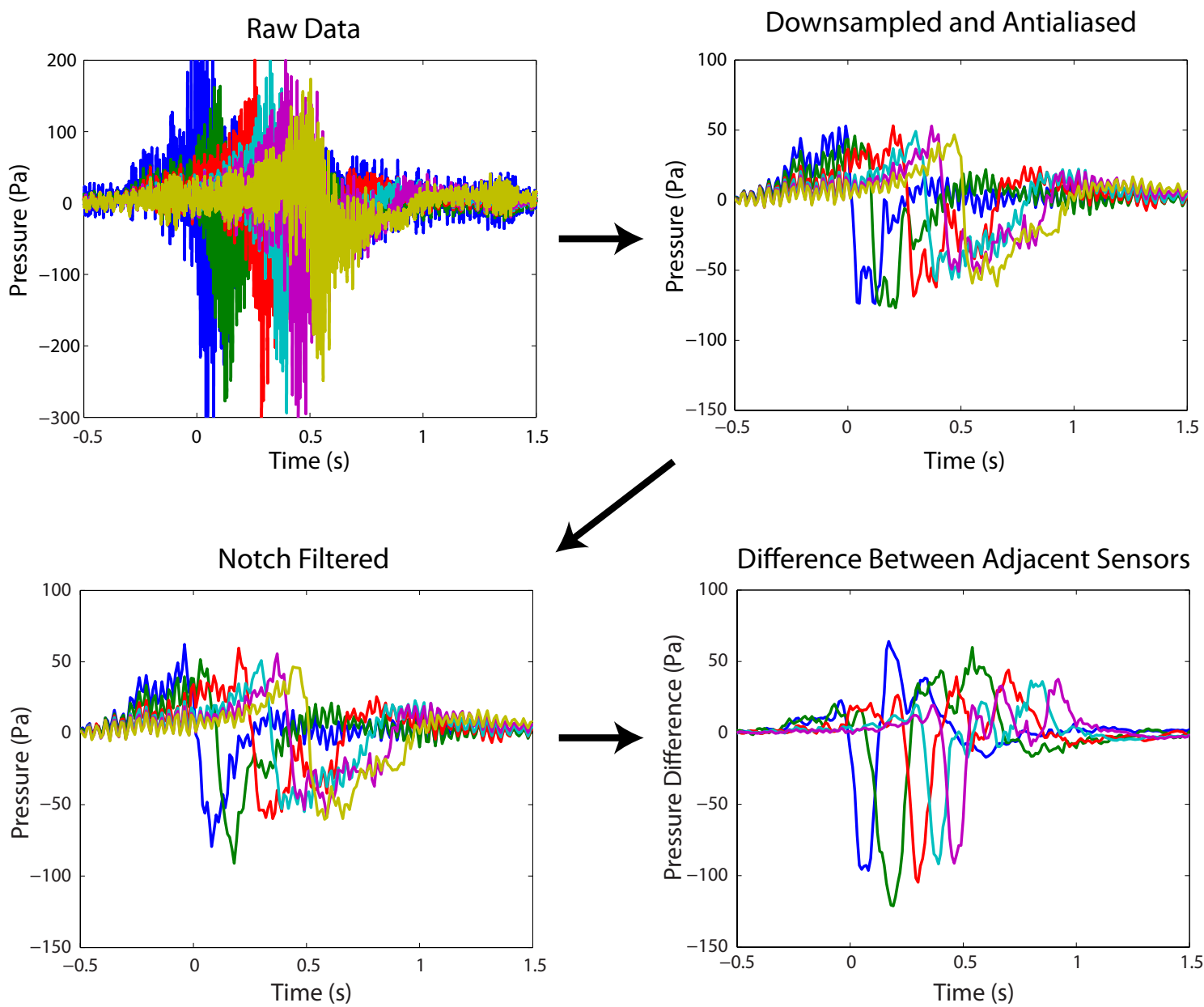

Difference Between Adjacent Sensors

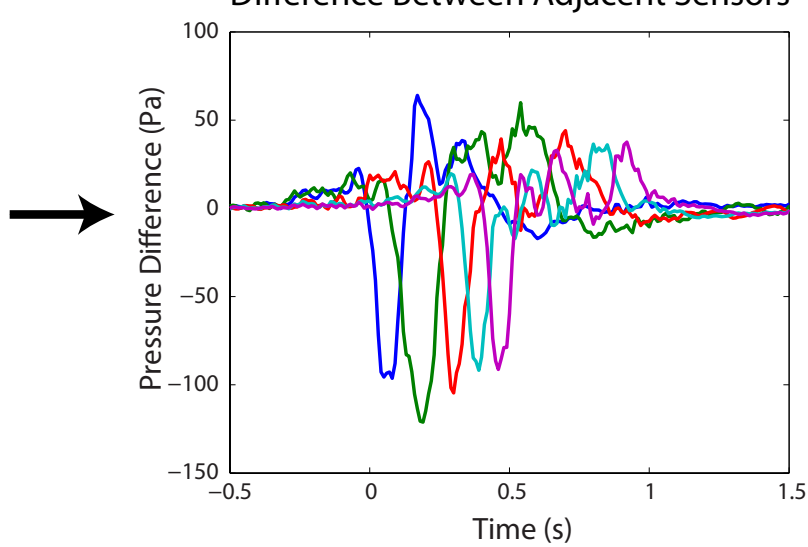

Figure 3-8: Noise processing. The pressure data is oversampled to avoid aliasing electrical noise, then antialiased and downsampled. A notch filter then removes the effect of cylinder vibrations. To avoid contamination from large wavelength water surface oscillations, the difference between adjacent pressure sensors is calculated.

Consistent with a dipole-like stimulus that would describe the vibration of the cylinder, the magnitude of the oscillation decays as the distance from the cylinder increases. This noise is removed from the data in post processing using a notch filter between 10 and $15 \mathrm{~Hz}$.

The final source of noise in the measurements is due to the standing waves in the surface of the tank. In terms of static depth, a change in the water level by $1 \mathrm{~mm}$ results in a $10 \mathrm{~Pa}$ difference. For waves there is a decay in the perturbation in pressure with increasing depth, as seen with linearized wave theory ([60]). However, the decay rate is correlated to the wavelength. In the experiments, the disturbances caused by the moving cylinder inevitably led to standing waves in the lowest mode of the tank, which took up to 30 minutes to die 
out. Considering the difference in pressure between adjacent pressure sensors, which can be interpreted as a simple spatial low-pass filtering scheme, effectively removes this noise source. The success of the scheme is owed to the fact that, since only the large-wavelength waves generate the disturbance, the disturbance is equal across all the sensors and therefore vanishes in the differential.

The final result of the combined post-processing is a data set consisting of the difference in pressure between adjacent sensors, sampled at $100 \mathrm{~Hz}$. In general, the processing is sufficiently cost-effective for expected applications outside of the laboratory, as the described filtering techniques can be realized through efficient algorithms in both software and hardware. Moreover, several of the post-processing steps are an artifact of the experimental setup and are unnecessary in an application setting. In particular, the first two filtering steps are necessary solely due to the noise introduced by the two dimensional gantry and not due to extra fluid stimuli (such as wake vortices). In either a biological or naval setting, one would not expect a moving object to generate noise comparable to the cylinder vibration or motor electrical interference. Signal contamination due to surface waves is still a concern in both applications, but it is notable that the fish lateral line also measures pressure differences.

\subsection{Results}

The results of the experiments match the CFD results in form. Generally described, the signal of a cylinder passing a sensor results in an initial increase in pressure relative to when there is no cylinder, followed by a sharp transition to negative pressure and a slower recovery to zero. The maximum pressure is generally smaller in magnitude than the minimum pressure after the sharp transition. This trace is similarly replicated, if shifted in time, for each sensor in a single run.

The averaged results for each point in the test matrix are visible in Figure 3-9. Based on these results, it is clear that there is a strong relationship between the velocity of the cylinder and the magnitude of the pressure signals, but a much weaker one between the magnitude and the distance of the cylinder. Instead, the distance of the cylinder appears 

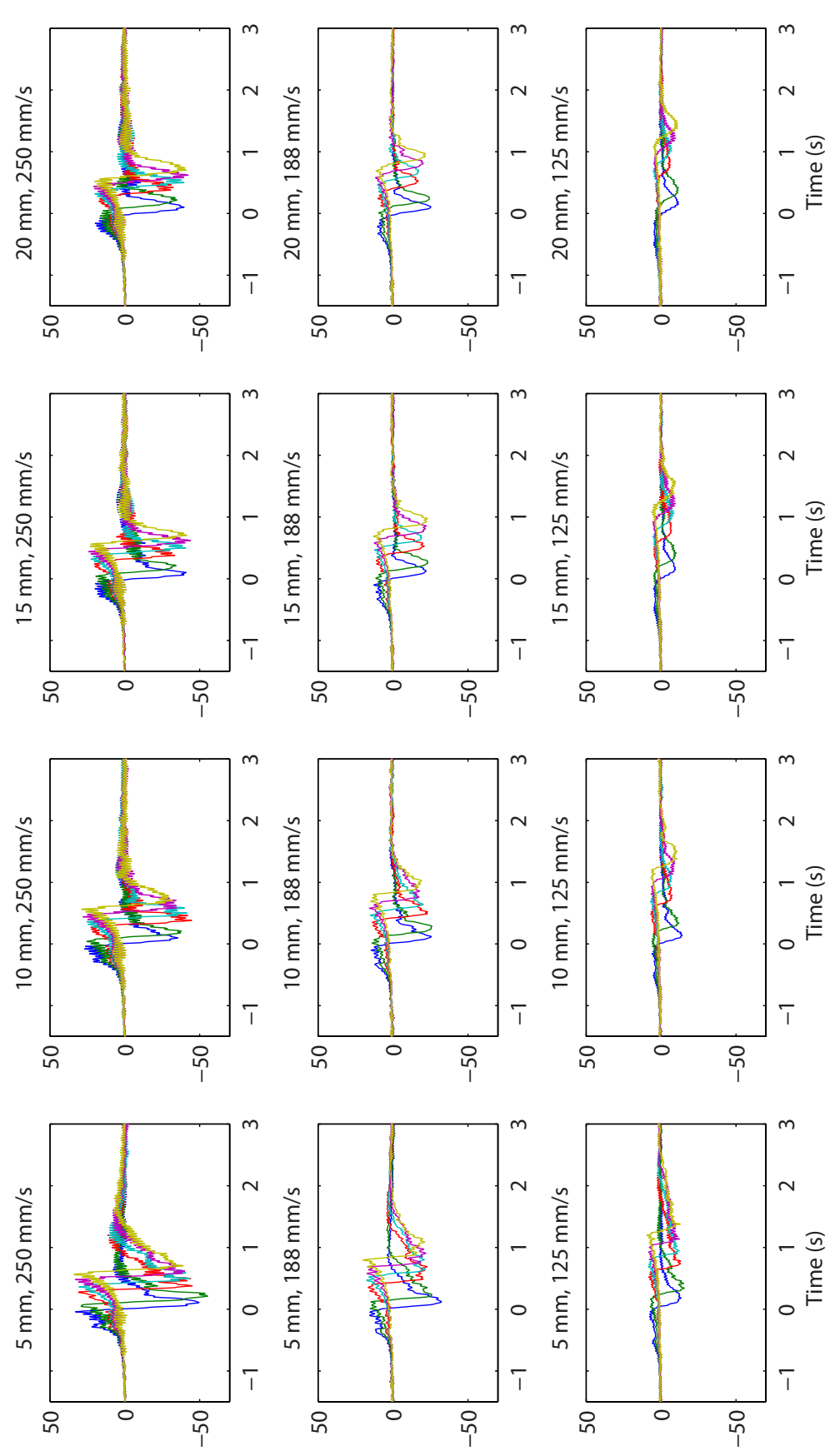

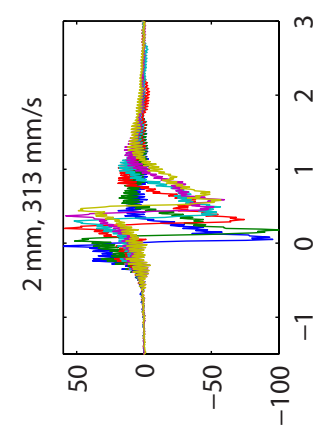

(ed) $ә \operatorname{exnss} \lambda_{d}$

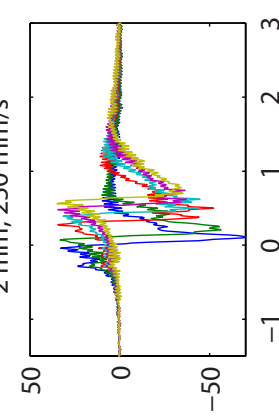

(ed) כגnssərd

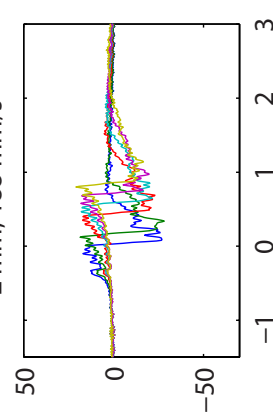

(ed) כגnssərd

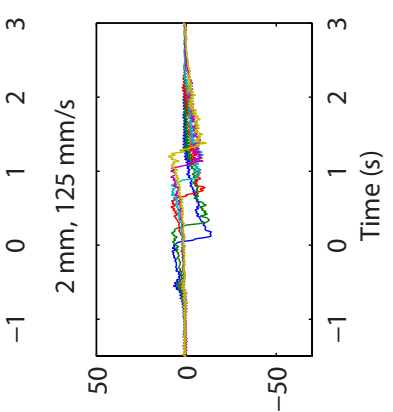

(ed) כגnssərd

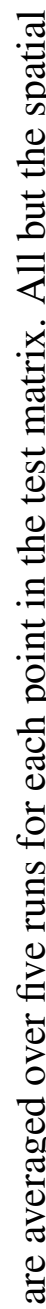

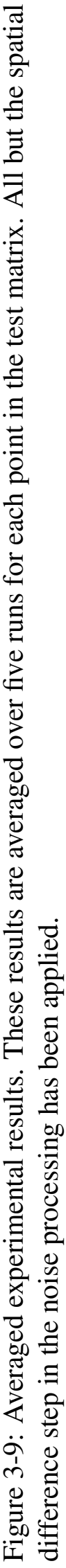


to primarily affect the speed at which the pressure recovers to it's ambient state after the cylinder has passed.

One important note is that the third pressure sensor from the left in the array was not available during the experiments due to electronic problems. This sensor is disregarded for the purposes of analysis and estimation. When considering the difference between adjacent sensors, this implies that one of these differences is taken over twice the distance of the others. However, this small anomaly requires no modifications in the model or estimation of the cylinder state, as the model is valid for a general number of sensors. Moreover, the loss of one sensor is very unlikely to have an effect on the overall results beyond decreased noise-reduction performance due to a loss of redundancy in measurement.

\subsection{Cylinder State Estimation}

With a promising model identified using CFD simulations and an experimental data set to use for evaluating effectiveness, it is possible to identify and test estimation techniques for tracking a cylinder position and size based on the measurements of a pressure sensor array. The HMM structure of the problem implies that many powerful estimation strategies, such as the Kalman filter, will apply. However, the nonlinear form of the model and the constrained relationships between the state variables mean that not all approaches will be equal and some exploration is required.

For the model described in this chapter (Section 3.2), the portion of the model dealing with the state transition (equation 3.1) is already linear. However, the other half of the model, using a Rakine half-body potential flow model to describe the measurements on the sensor array given a cylinder state (equation 3.2) is not linear. Many estimation techniques, such as the Kalman filter, work directly on linear models. For these techniques, the success of local linear approximations to the model, such as those in the extended Kalman filter, depends on the type and extent of the nonlinearity. Considering the experimental setup in which the sensors are imbedded in a flat wall, the relation between the measurements and the state is:

$$
\underline{P}(\underline{s})-P_{\infty}=\frac{1}{2} \rho u_{n}^{2}\left|1+\frac{k_{n}}{\underline{s}-z_{n}}+\frac{k_{n}}{\underline{s}-\overline{z_{n}}}\right|^{2}
$$


Both the pressure $\underline{P}$ and sensors location $\underline{s}$ are vectors in these equations. Also, since the sensors are imbedded in the flat plate that forms the axis of symmetry, $\underline{s}$ is a real vector. The overbar on $\overline{z_{n}}$ denotes the complex conjugate. Note that the pressure is given as a pressure difference with respect to the far-field pressure $P_{\infty}$. Also, the variable $k_{n}$ is related to the radius of the modeled cylinder, but due to the presence of an image, it also depends on the distance of the center from the sensors, $y_{n}$. The exact relation is:

$$
k_{n}=\frac{1}{2} \frac{\sqrt{4 y_{n}^{2}-r_{n}^{2}} a}{y_{n}}
$$

As expected, the variable $k_{n}$ approaches the radius $r_{n}$ as the distance $y_{n}$ increases. Although the radius, speed, and perpendicular distance are constant in the experiments, the model allows for changes as might occur under different circumstances. The combination of these equations fully describe the measurement model, and result from the Rankine half-body model, equation 3.2, applied to the Bernoulli equation, equation 3.4. Based on equations 3.7 and 3.8, it is clear that the pressure measurements are nonlinear in all of the state variables.

\subsubsection{Cylinder Tracking via a Kalman Filter}

The Kalman filter is a form of a predictor-corrector estimator which chooses a gain for the correction which is optimal for a linear system with white Gaussian noise. It operates on a hidden Markov model or its continuous state equivalent. To estimate the hidden state based on the measurements, the Kalman filter tracks both the state estimate and the error covariance matrix of the estimate. In dealing with nonlinear transition and measurement models, the most common approach is to linearize the equations in a first order taylor series approximation, referred to as the extended Kalman filter. Another more recent extension of the Kalman filter, the unscented Kalman filter, effectively keeps higher order terms of the approximation with the added advantage of not having to compute the Jacobian each time. As a very flexible and robust estimation technique, the Kalman filter and its variants are the first logical estimation approaches for consideration. The details of the Kalman filter and its nonlinear variants are described in [27] among many other texts. 
As it relates to the problem of tracking the state of a moving cylinder, a key component of the Kalman filter approach is that it approximates all noise variables as zero mean Gaussian random variables. There are two sets of noise variables. The first set is associated with the measurement equations. In this case, the distribution can be matched to the electrical noise inherent in the measurement. The second set of noise variables is those represented in equation 3.1 which capture any uncertainty in the transition between states and are independent from the measurement noise variables. While the transition between states is nominally known exactly, this noise allows for some flexibility due to a potential misalignment in the axis of motion or the influence of unmodeled factors. The variance of these noise variables also affects the gain used during the Kalman filter correction step. In tuning the relative weight of the transition noise variance to the measurement noise variance, it is possible to adjust the confidence in new measurements of the Kalman filter relative to the predicted trajectory based on the state transition model.

The state variables in this problem are not independent. In particular, both the radius and the perpendicular distance must be positive, and the radius of the cylinder cannot be greater than the perpendicular distance: $0<r_{n}<y_{n}$. These constraints are physically intuitive, since the cylinder cannot pass behind the sensors and a negative radius is nonsensical. Similarly, if the radius is larger than the perpendicular distance, this implies that a collision will take between the cylinder and the plate on which the sensors are mounted. Unfortunately, the potential flow model used to predict the pressure measurements along the linear sensor array cannot account for these physical boundaries. The boundary that defines the the surface of the cylinder is a hypothetical one, matching a streamline in the flow model at the stagnation point only. As a result, the equations described in 3.7 and 3.8 result in predicted pressures of similar magnitude regardless of the signs of the radius and distance, and more importantly, regardless of the relative values of the two variables. This implies that there is no substantially increasing error that will drive the estimate of the Kalman filter away from these boundaries in the correction phase of the filter, and as a result the estimated state may stray into non-physical regions. These occurrences can be crudely prevented by testing the state variable relations each iteration and forcing the state back into a legitimate physical region when a violation occurs. This can work if it occurs 
rarely, but for the most part does not lead a good result as it does not stop the underlying cause.

The extended Kalman filter was tested first on data simulated using the model from section 3.2 and subsequently using data gathered in the experiments. For the model described in this chapter (Section 3.2), the portion of the model dealing with the state transition (equation 3.1) is already linear. The measurement equation is linearized using the corresponding Jacobian matrix. The simulated data was generated by first generating a sequence of states using the state transition model initialized to a state in line with the experiments. For example, with an initial state of $35 \mathrm{~mm}$ perpendicular distance, $15 \mathrm{~mm}$ radius, $250 \mathrm{~mm} / \mathrm{s}$ velocity, and $-50 \mathrm{~mm} \times$ position, the sequence of states would progress in the $\mathrm{x}$ direction with constant increments determined by the sampling rate, with the other variables held constant. Based on these states, the measurements are generated using equation 3.7, again without the addition of any noise. This noiseless simulated data set is the most favorable initial test for an estimation algorithm, since the model used in the algorithm exactly matches the model used to generated the data and there is no noise. As such, it provides an opportunity to tune the various noise and initialization parameters of the Kalman filter. Although efforts were made to accurately reflect the noise parameters of the experiment, little information is available for the process noise in the state transition, and so the sensitivity of the measurements to the state variable can be used to influence the choice. A typical result of the extended Kalman filter application to simulated data can be seen in Figure 3-10. In this example, it is evident that the extended Kalman filter converges to fairly accurate estimates of the position and radius, but underestimates the velocity. The exact behavior of the estimate convergence in extended Kalman filter depends heavily on the initial state. In particular, it was found that severly underestimating the initial guess for the radius, and overestimating the initial perpendicular distance, are important for the accurate convergence of the state in the extended Kalman filter. However, with a reasonable choice of initial condition, the extended Kalman filter reasonably extracted the state of a moving cylinder from simulated data.

The experience with the simulated data sets also allowed the fine tuning of the noise model used in the transition equations. Although the overall ratio of transition noise vari- 
ance to measurement noise variance affects the confidence applied to new measurements, there are still relative weights in the noise variables to consider. In the case of the measurement noise, there is no reason to believe that any of the off the shelf sensors behave differently once they have been calibrated. In addition, following the data preparation described in $3-7$, there is no correlation between the noise of individual sensors remaining. Therefore the noise covariance matrix for the measurements is in the form of a constant times the identity matrix. It is slightly more complicated in the case of the covariance matrix for the state variables. In this case, there is no guiding physical intuition, since the noise does not represent a physical uncertainty in the state transition. In fact, it is possible to set the noise variance of the state variables to zero and have results very similar to those of figure 3-10. However, this leads to some very undesirable behavior if the cylinder does not pass the pressure sensors shortly after the Kalman filter is initialized. With zero noise variance in the state variables, the reliance on the measurements will decrease with time to the point that new measurements will be effectively ignored. This is a problem if it occurs when the sensors are effectly only measuring noise since a large range of state configurations can achieve this result. By the time the sensors pick up the effect of the cylinder, the estimate may have hardened into a very small cylinder, a cylinder far away, or a stationary cylinder, which all could have resulted in no measurement. By including a nonzero noise covariance matrix for the state transition equation, one effect there is an upper limit induced on the confidence of the estimate, so that the filter will respond to new measurements. Given that the state covariance should be non-zero, I assumed the covariance matrix to be diagonal. It was observed from the responses to simulated data that the variance of the $y$ position and the radius should be smaller than that of the $\mathrm{x}$ position and velocity, in order to converge well under a variety of conditions while avoiding the nearby boundaries. Based on the experience with the simulated data, a ratio of 0.5 between the state noise variances worked well.

Complications due to the firm relationships between the state variables are not clear until the estimation algorithm is applied to experimental data. In the experimental data, the Rankine model used as the base of the Kalman filter is only an approximation to the physical relation between the cylinder state and the pressure measurements. As earlier de- 

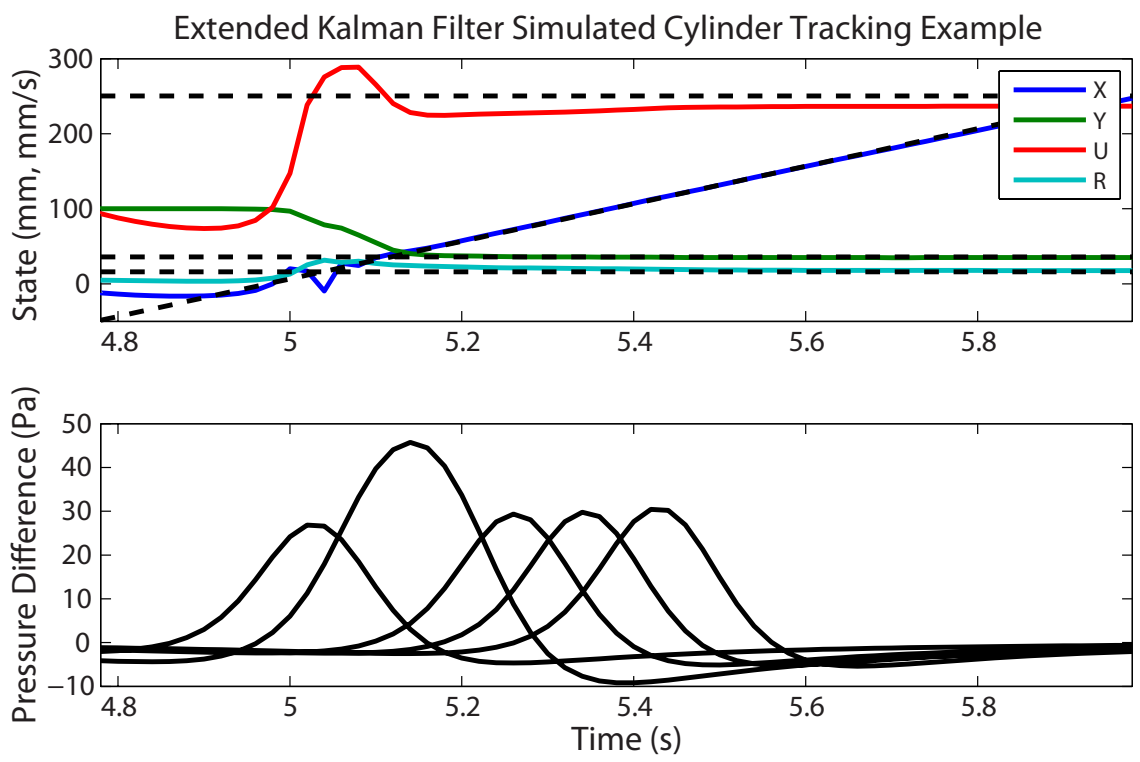

Figure 3-10: Extended Kalman filter cylinder tracking simulation. The result from the estimation of the cylinder state based on data generated with the same model used in the Kalman filter. The top figure compares the estimated state (in color) to the true state (dotted black). The bottom shows the simulated pressure data that the extended Kalman filter is acting on.

scribed, there are known discrepencies between the pressure from the Rankine model and the viscous CFD simulations in the region upstream of the cylinder. Similarly and more importantly, the wake region downstream of the cylinder only resembles the pressure generated from the Rankine model on average, not for any particular time instant. In addition to this, other unmodeled fluid eddies may contribute to the pressure measured in the experimental runs. The result is that there are disturbances in the pressure measurements which are not directly accountable in the Rankine model. It appears that these differences between the model and reality cause the estimate to drift into non-physical states. This is evident in Figure 3-11, where the forced corrections in the perpendicular distance are evident. In this example, the Kalman filter is initialized very close to the true location, demonstrating the that extended Kalman filter cannot track the size and position of a cylinder based on experimental data.

In order to test whether a poor handling of the nonlinearities of the model was the cause for the poor performance, the unscented Kalman filter was implemented on the same 

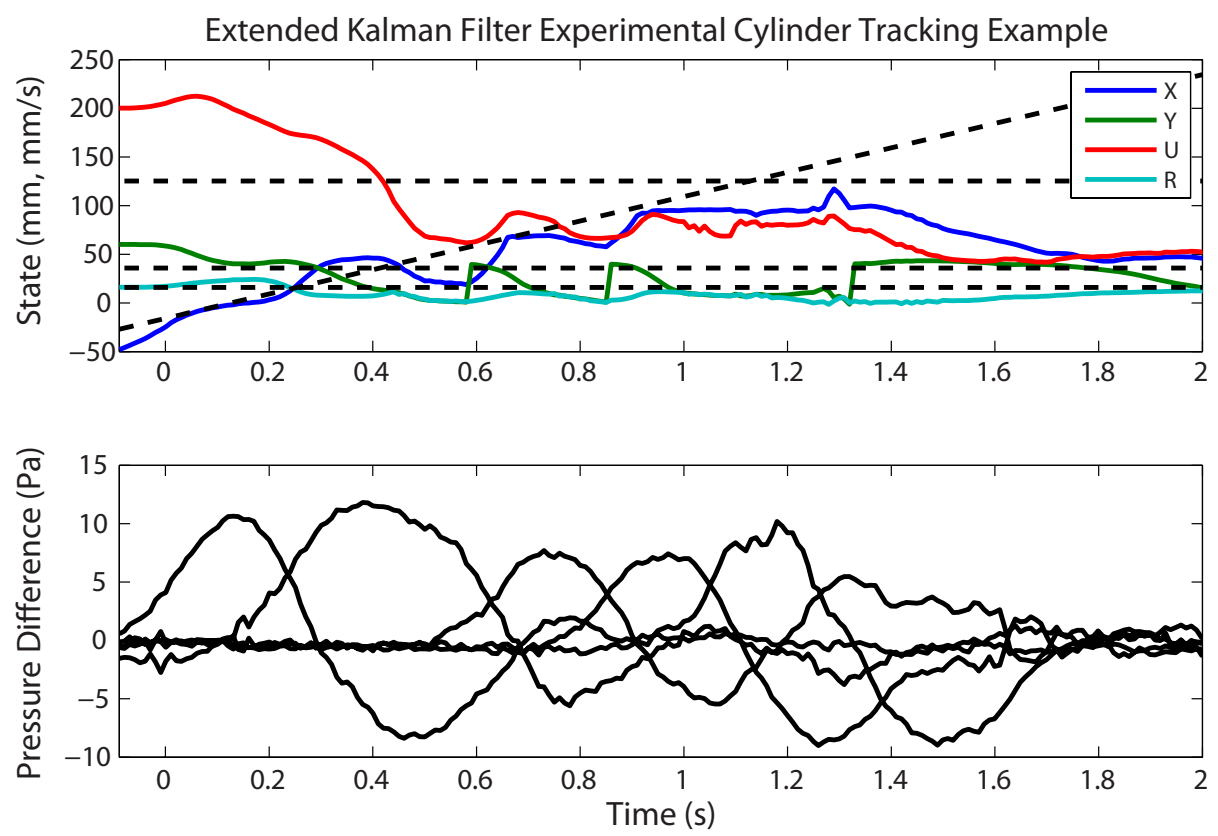

Figure 3-11: Extended Kalman filter cylinder tracking on experimental data. This figure is in the same format as Figure 3-10, but the data which the extended Kalman filter is using for the tracking was gathered from one of the experiments. Note the three forced corrections in the $\mathrm{Y}$ estimate. This result is representative of the extended Kalman filter when applied to other experimental data.

data and model. As mentioned, this variant of the Kalman filter generally has improved performance over the extended Kalman filter [41] since it utilizes a better approximation of the nonlinearity in the model. The results are very similar to those from the extended Kalman filter.

In summary, both the extended and unscented variations of Kalman filter are unable to track the state of a moving cylinder, composed of the position, radius, and velocity, based on an array of pressure measurements and an analytic model as described here. The primary cause appears to be a combination of the strict relations imposed on two of the state variables and the approximate nature of the analytic model. Sections of the data in which cannot match the model well result in non-physical state estimates and cause the rest of the estimate to diverge. Attempting to correct this by an automated restarting or resetting of some state variables does not solve the problem as areas of disagreement between the data and model occur too frequently. 


\subsubsection{Cylinder Tracking Via Particle Filter}

In order to overcome the pitfalls that trap the Kalman filter, it would be ideal to limit the space of acceptable state vectors to physical situations in a manner that is inherent in the estimation method. In the Kalman filter, the assumption of Gaussian noise in the state transition model implicitly assumes that each state variable has a domain of the real numbers, since that corresponds to the domain of a Gaussian random variable. One approach to limiting the space of state vectors is therefore to modify the noise parameters such that the probability distributions of the state variables are only non-zero over physical values. The particle filter is an estimation algorithm that allows this sort of specificity in the probability distribution that operates on the familiar hidden Markov model structure.

The basic approach of the particle filter estimation method is to independently track a number of possible states, each possible state referred to as a particle. Each particle is associated with a weight. The combination of the particles and corresponding weights forms a discrete approximation to the probability distribution of the state given all previous and current measurements. For example, the expected value of a state variable can be computed by $\sum_{i} w_{i} q_{n}^{i}$ where $i$ refers to the particle number, $n$ the timestep, $w$ the weight, and $q$ one of the state variables. At each new timestep, the particle is updated by randomly drawing a new particle from a probability distribution based on the old particle (and potentially the new measurement). The weight of the new particle is subsequently updated using the likelihood of transitioning to the new state and the likelihood to make the current measurement from the new state. Therefore, if the particle was randomly chosen far from it's expected new position or if the new particle is very unlikely to have generated the pressure measurements, the weight associated with that particle would be reduced. Since the particle filter operates as a discrete approximation to the conditional distribution of the state, the number of particles influences the accuracy of the estimate. It therefore frequently requires a large number of particles to obtain successful results, which may be computationally expensive. In another difference with the Kalman filter, the particle filter relies on randomly choosing an updated particle from a probability distribution, so the resulting estimate will not be reproduced identically if the algorithm is repeated on the same data. This is also dependant 
on the number of particles, with the effect reduced if there are many particles covering the state vector space.

There are many different variants to the particle filter algorithm, just as for the Kalman filter. However, because of the Monte Carlo foundation of the particle filter these variants are unrelated to the non-linearity of the problem which is handled without trouble. Instead, the variations of the particle filter are related to the sampling method when transitioning from one state to the next. New particles are drawn from a probability distribution termed the proposal distribution which is ideally equal to the conditional probability of the new state conditioned on the previous state and new measurements $p\left(q_{n} \mid q_{n-1}, m_{n}\right)$. Given the complicated relation between the measurements and the state, this conditional probability is difficult to know. The most common variant of the particle filter uses a proposal distribution based only on the state transition equations $p\left(q_{n} \mid q_{n-1}\right)$ called the bootstrap method. Other approaches use an extended or unscented Kalman filter to approximate $p\left(q_{n} \mid q_{n-1}, m_{n}\right)$ for the proposal distribution. The focus on this chapter will be on the bootstrap particle filter. The unscented particle filter was also implemented, but it led to increased errors and computation time without any compensating benefit. The details of the bootstrap particle filter method are further described in [67].

The key difference between the Kalman filter approaches and the particle filter, as far as tracking a moving cylinder is concerned, is that the range of boundaries in the state variables can be explicitly included. This is through the specification of the distribution of the noise random variables in the state transition equation (3.1). The distribution of the noise for the $\mathrm{x}$ position and velocity state variables is the same as was implied for the Kalman filter: zero mean Gaussian distributions with some specified variance. Both the y position and the radius need different distributions for their corresponding noise variables since they have restricted ranges.

In the case of the y position, the probability distribution needs to be positive, but one would expect that if the current state is sufficiently far from zero relative to the standard deviation, then the distribution would be roughly Gaussian. That is to say, the desire is that, far from zero, the distribution would be symmetric and zero mean with a single peak and long tails. The log normal distribution fits these characteristics well. The log normal 

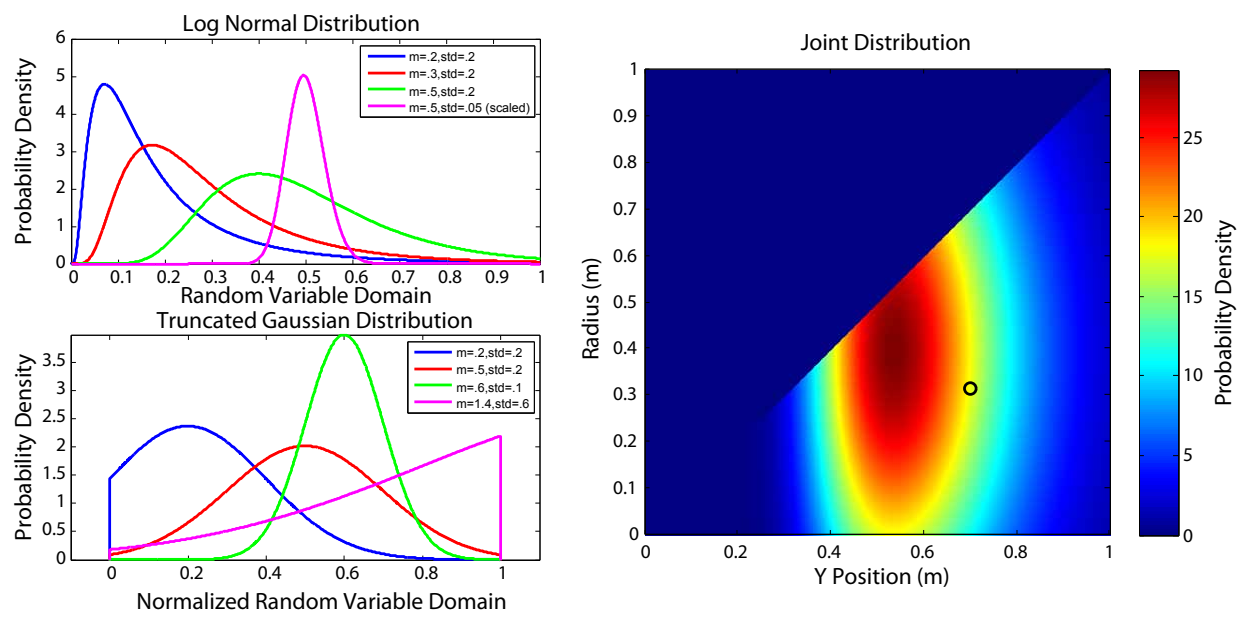

Figure 3-12: Non-Gaussian State Variable Distributions. The lognormal distribution and a truncated Gaussian distribution, corresponding to the y position and radius respectively, are shown at left for various parameter values. Their joint distribution is shown at right.

distribution is parameterized by two variables, denoted by $a$ and $b$, which correspond the mean and standard deviation of the natural logarithm of the random variable. For the purpose of representing the y position noise, the mean and standard deviation of the random variable itself are more relevant, since the random variable will have zero mean. The expected value of a log normally distributed random variable is $m=e^{a+\frac{1}{2} b^{2}}$ and the variance is $v=\left(e^{b^{2}}-1\right) e^{2 a+b^{2}}$. Solving these equations for the noise parameters results in:

$$
a=\log \left(\frac{m^{2}}{\sqrt{m^{2}+v}}\right), b=\sqrt{\log \left(\frac{v}{m^{2}}+1\right)}
$$

One note on this result is that since the mean of the distribution is not generally the same as the maximum likelihood. Depending on the relationship between the standard deviation and the mean, the maximum likelihood will be significantly less than the mean, and the distribution will appear skewed away from zero (see Figure 3-12). This is important in the long run because it means that the expected value of the distribution should be used as the estimate of the state variable, not the maximum likelihood value.

The physical range for the radius of the cylinder is bounded by zero below and by the y position above. While there are bounded distributions such as the Beta distribution which could be used to model this noise variable, they do not capture some of the intuitive 
behavior required. Clearly the expectation is that the cylinder radius does not change. Therefore if the previous cylinder radius estimate is not near either boundary in comparison to the standard deviation, the noise variable associated with the update should be zero mean and confined. In this situation, a Beta distribution or Gaussian would work effectively and the boundaries would not come into play. If the estimate of the radius is very small or near the estimated y position compared to the standard deviation, then the distribution should to be asymmetric with a bias away from boundary, but it would be logical if the most likely value was still the previous radius. Again the Beta distribution is flexible enough to capture this well. One further issue stems from the choice that the y position is free to be updated over it's complete range instead of being limited below by the radius. This implies the possibility of an updated y position that is smaller than the previous radius. In this case, the reasonable form of the probability distribution would be to have the maximum likelihood at the upper boundary of the distribution, and to have the rest of the distribution monotonically decrease. Here the Beta distribution has more difficulty. One solution that achieves all of the desired behaviors is a truncated Gaussian distribution. This corresponds to using a Gaussian distribution centered at the previous value with a fixed variance, but fixed at zero outside the physical values and normalized so that it integrates to one (Figure 3-12). In addition, this approach implies that the farther the physical range is from the previous value, the flatter the distribution will be.

Much like in the case of the Kalman filter, the determination of the state transition equations with their noise random variable distributions still leaves some parameters governing the width of the distributions free. As for the Kalman filter, these parameters are not representative of any actual stochastic behavior in the state transition. However, the effect of these parameters is substantially different than for the Kalman filter. The particle filter works best with a lot of particles of equal weight, but the tendency is to converge in weight to just a few particles which offer the best estimate. The standard approach to dealing with this, used here, is to resample the particles from the estimated posterior distribution when the number of effective particles falls to low (See [67]). An important consequence is that after resampling, many of the particles have the same state until the next update. The speed at which the particles subsequently disperse is governed by the variance of the noise vari- 
ables. If the variance is too high, the particles will disperse too quickly and the effective number of particles will drop immediately again, leading to a very inconsistent estimate. If the variance is too low, then it may take a long time for the filter to sample an adjacent space that may offer a better estimate. Therefore the noise variance should in general be as small as reasonable such that the estimate converges quickly.

Using simulated data in the same way as for the Kalman filter, the noise variance associated with each of the four state variables was tuned using experimental runs on idealized data. In this case, the sensitivity of the pressure measurements to the state variable was used as a guide for the variance associated with that variable. This is because the more sensitive the measurements are, the more likely the possibility of converging strongly to an incorrect value during resampling, especially once it is applied to experimental data where there is some model mismatch. Both the $\mathrm{x}$ position and the velocity were found to be the most influential on the pressure measurements. The radius is also fairly influential, but is confined between zero and the y position and therefore does not need to explore as large a sample space. Based on many test runs with simulated data, the standard deviations of 5 $\mathrm{Pa}, 2 \mathrm{~Pa}, 5 \mathrm{~Pa}$, and $2 \mathrm{~Pa}$ for the $\mathrm{x}$ position, y position, velocity, and radius respectively were found to work well.

In addition to the state transition noise characteristics, the noise variance for the pressure measurement model was also specified using the simulated data as a test case. In the simulated data, no measurement noise was used to examine the convergence under optimal conditions. For the actual pressure sensors, the amount of noise after processing for electrical and static pressure effects (Figure 3-8) is very small in comparison to the meaningful signals. Using a measurement noise standard deviation that matches the data leads to problems with the Kalman filter algorithm, since it quickly causes the effective number of particles to decrease. Since the model being used is not perfect, it is a problem if during a timestep in which the model does not fit the measurements well due to either external factors or inadequacies of the model, the entire particle distribution converges during resampling on a single particle that does fit the measurements. This situation effectively derails the estimate. To avoid this, the standard deviation of the measurement noise is set to $15 \mathrm{~Pa}$, which is sufficiently broad that the effective particle number collapse is reduced 
while still increasing the weight of particles that correspond well with measurements.
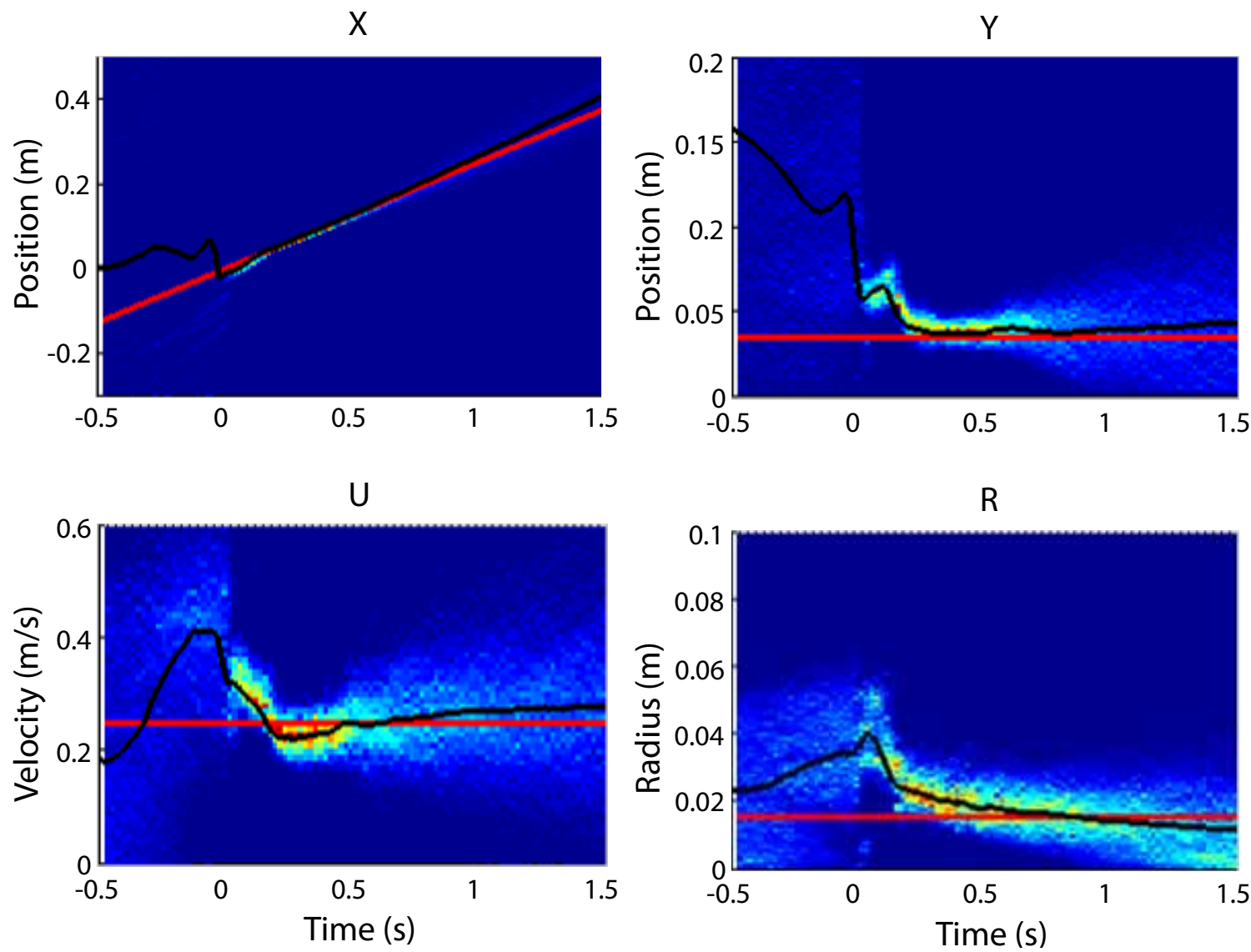

Figure 3-13: Results from particle filter on simulated data. The estimated probability distribution based on the history of measurements is displayed for each timestep for the four state variables. Each vertical slice is therefore normalized to sum to 1 . The black line denotes the expected value of the variable as calculated from the state estimate distribution. The red line is the true state used to generate the pressure measurement data.

A representative result of the particle filter applied to simulated data is seen in Figure 3-13. Since the particle filter tracks a discrete approximation to the probability density function (pdf) of the state conditioned on all the previous measurements, this figure displays the full pdf for each timestep. The color scale of blue to red corresponds to zero to high probability density. Since each vertical slice must integrate to one, the maxima are not comparable in value from one timestep to the next. On the other hand, viewing the full pdf gives a complete sense of the confidence in the estimate. For the example discussed here, one thousand particles were used in the particle filter. The expected value of the state 


\section{Simulated Pressure Difference}

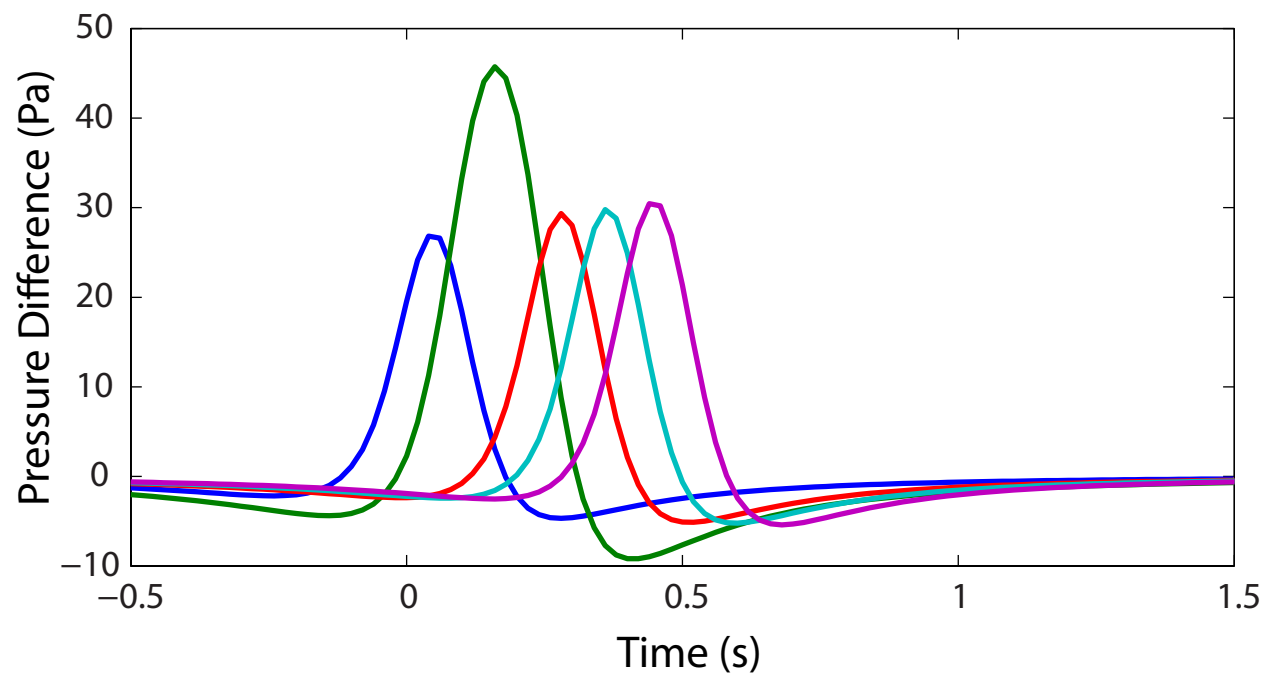

Figure 3-14: Simulated pressure corresponding to the particle filter estimate. This pressure difference time history is generated using the pressure measurement model based on a Rankine half-body following a state path that follows the state transition equations. No noise is included in the measurement or the state transition, resulting in a test case that offers the best conditions for estimation.

variable at each time instant is shown in black. The estimated cylinder state depicted in Figure 3-13 is based on the data shown in Figure 3-14. As with the Kalman filter, this data was generated from a known state path using the same measurement model as in the particle filter. That known state path is depicted in red in Figure 3-13. Since the data is noiseless in both the state transitions and the measurements, it represents an idealization of the problem in which the filter can be tested under its optimal conditions. To match the experimental data later, the third pressure sensor in the linear array is removed before computing the pressure difference.

The results depicted in Figure 3-13 show that a particle filter successfully tracks position, radius, and velocity of a moving cylinder in the optimal simulated case. There is no strong dependence on a single initial condition as in the case of the Kalman filter, and by the underlying noise model there is no chance of a non-physical state being reached. Given this ideal test case, there are a number of features evident in the way the cylinder is tracked by the particle filter. First, the a priori probability distributions for the state variable were initialized to be uniformly distributed over a generous range (-0.5-0.5m for the $\mathrm{x}$ direction, 
for example). In examining the results, this initialized distribution is essentially unchanged until the cylinder is in front of the first sensor at time zero. At that point the estimate coalesces to an estimate that is initially still incorrect. The cause of that initial coalescence can be identified by comparing the time with that in Figure 3-14. There is little influence on the particle filter until a substantial deviation from zero is observed in the pressure difference between the sensors. Immediately following the coalescence there is a systematic error in the estimate for all the state variables, though it is less pronounced in the $\mathrm{x}$ direction. This transient error is possibly due to particles not covering the relevant portion of the sample space when the algorithm resamples. Given the large number of particles in this example, though, it is likely due to an ambiguity in the measurement likelihood. The time for the estimate to converge to the true state values is roughly the same as for the pressure difference between the third and fourth pressure sensors to increase past the measurement noise standard deviation. This implies that the difference between three sensors only may not be enough to uniquely identify the path of the cylinder states. After the pressure measured at the sensors returns to zero, and thus the pressure difference as well, the estimated state slowly diverges from the true state. This is not unexpected since again there are many possible states which give zero pressure measurements. In this region, the effect of the state noise variable variance is visible in how the distributions widen, particularly for the $y$ position and velocity.

When applied to the experimental data set, the ability of the particle filter based on a Rankine half-body model to track the state of a cylinder is similar to the simulated data results. The results of two experimental runs are displayed side by side in Figure 3-15. These two experimental runs represent typical estimated traces for runs with high signal strength (left column) and low signal strength (right column). The experimental results match the behavior observed in the simulated data, with the initial uniform distribution being largely unchanged until a signal is measured by the first sensor and a broadening of the estimated state distribution once the cylinder has passed the sensors and the signal is reduced or absent. Unlike the simulated results, the state estimate diverges more substantially after the cylinder has passed in the experimental data. This is unsurprising given that the pressure measurement model used in the particle filter is based on an average response 
in the CFD simulations. For a single experimental run, pressure measured in the wake region is expected to differ from the model therefore causing a change in the estimate. There is some indication of the delayed convergence that was observed with the simulated data, particularly in the $\mathrm{Y}$ position and radius of the low signal example. In that case, it appears that after signals are initially measured by the first two pressure sensors there is a period in which the $\mathrm{Y}$ position and radius are significantly over-estimated. This changes at about the time pressure difference between the third and fourth pressure sensors increases. In contrast, the strong signal example has a much smaller shift, if any.

The particle filter based on Rankine half-body tracks the cylinder well in general, but there are differences in the estimation accuracy for each state variable. The $\mathrm{x}$ position state, parallel to the direction of the linear sensor array, is tracked best. However, unlike the simulated results, the estimate of the $\mathrm{x}$ position shows a step-like progression which is substantially more pronounced in the strong signal example. The level portions correspond in number and location to the peaks in the pressure difference measurements. Another notable observation is that both the y position and the radius are under-estimated. This is somewhat expected for the radius since in choosing the simpler potential flow model of the Rankine half-body, it was noted earlier that the response of the slightly more accurate dipole-source model could be reproduced by reducing the radius in the Rankine half-body. The under-estimation of the y position is a little more surprising. It appears to be more significant in the example with a smaller signal strength, but it is unclear whether this is associated with the underlying model or a lack of sensitivity in the measurements due to the y position. There is some error in the velocity estimate, though in general probability distribution for the velocity is broad. Keep in mind that the velocity is not being calculated based on a cross-correlation of the signals, which would provide a much more accurate velocity estimate after the fact, but would not be able to attempt to track changes in velocity in real time.

The results displayed in Figure 3-15 are based on a particle filter with one thousand particles. This amount is useful for obtaining a good image of the conditional state distributions, as shown in the figure. However, it also generates an excessive amount of computation time (order $15 \mathrm{sec}$ ). Reducing the number of particles will not immediately effect the 

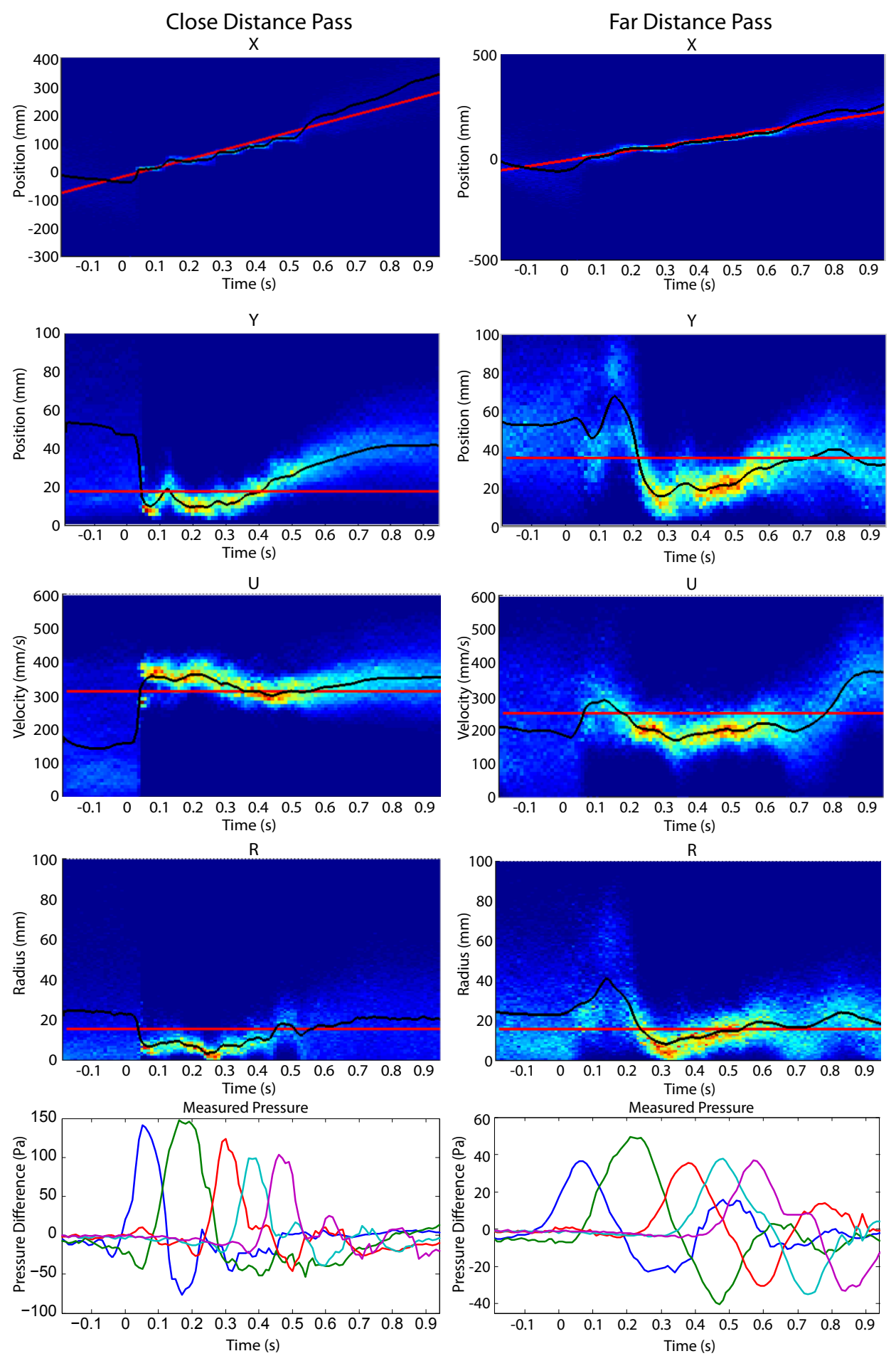

Figure 3-15: Particle filter estimation on two experimental data sets. The estimated results are shown for high and low signal strength examples from the experimental test matrix. The format of the state estimates is the same as for Figure 3-13. The corresponding pressure difference data is shown for reference against the estimate behavior. 
expected value of the distribution, which is used as the estimated state. At a certain point, there are two effects of having too few particles that will cause difficulty. First, the particle filter may not converge to the correct values since the sample space may not be covered sufficiently at the outset. This can be slightly counteracted by narrowing the initialization distribution of particles using external information. Similarly related, the stochastic nature of the particle filter is aggravated if there are insufficient particles. Since the sample space is not well sampled, which ever particle is closest to the true state will be the point of convergence during resampling. The randomness of this particle means that different runs of the particle filter will substantially different results. In order to choose an adequate number of particles for tracking a cylinder reliably but in the least possible time, the behavior of the particle filter was systematically studied on the experimental data set. The results for one of the experimental runs, at $200 \mathrm{~mm} / \mathrm{s}$ and $20 \mathrm{~mm}$ away from the sensor array, are visible in Figure 3-16. In this figure, the bias refers to the mean error between the true state and the estimated state during the period in which the sensor array is measuring the influence of the cylinder. In addition, a small convergence time is removed from consideration at the start. For each data point, ten different passes of the particle filter were used. For this set of experimental parameters, it is fairly clear that there is a leveling off in the improvement provided by increasing the number of particles at around 150 particles. If only interested in the estimate via the expected value, 150 particles should be sufficient. A very similar picture is present when considering the standard deviation among the 10 trials for each point (Figure 3-17). These results vary slightly from one experimental run to another, so 300 particles are used subsequently to ensure being beyond the transition point.

In order to examine the effect which the experimental parameters might have on the ability to estimate the estate of a moving cylinder, the full test matrix of experiments was tracked using the particle filter as described with 300 particles. In particular, this will examine the dependence of the ability to identify each of the state variables against the distance and velocity of the cylinder. Running the particle filter based on the Rankine half-body once for each of 5 experimental runs at each point on the test matrix generates Figure 3-18. The error in this case refers to the mean difference between the estimated state and the true state over a period during the later portion of the cylinder's pass by the sensors. 


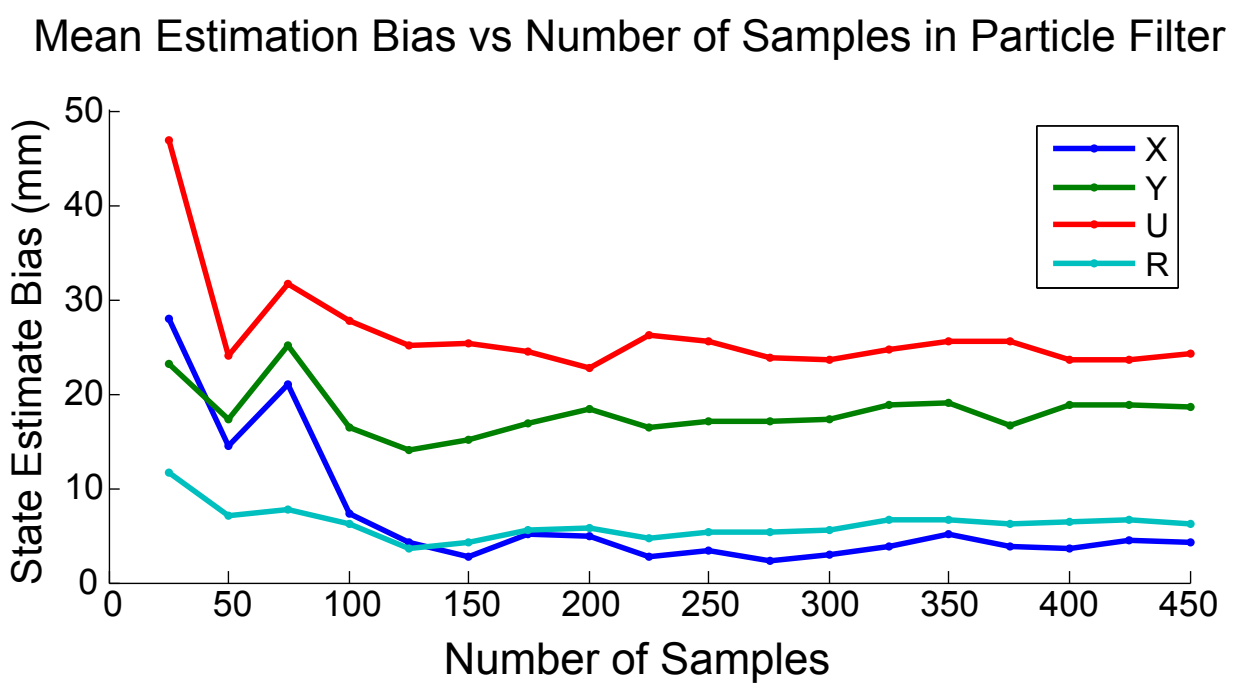

Figure 3-16: The dependence of error bias on particle number. The absolute average error, calculated on the time when the pressure difference magnitude is above $10 \mathrm{~Pa}$ after some initial convergence time decreases as the number of particles used increases. Each point is an average of 10 different runs of the particle filter.

The start of the test period is a brief 0.2 seconds after the cylinder passes the first sensor, and the end is when the signal strength on the sensor array decays. The absolute value of this mean error is then averaged over the five data sets in order to generate a point in the figure.

In examining the error landscapes described by the figure, there appears to be less effect from the velocity than would be initially expected. Since the speed of the cylinder affects the wake and the amount of time the sensors measure signals from the cylinder, it would be logical to expect the ability to track a cylinder to degrade as the speed increases. This was not observed in the data over the ranges in velocity tested, with the possible exception of the error in the $\mathrm{x}$ position when also at large distances.

With respect to distance, there are two competing possible causes for error. The model used in the particle filter was found to work well in open water and adequately when near a wall. However, if the cylinder passes very close to a wall, there are likely other unmodeled flows that are generated by the interaction beyond a simple deformation of the wake. At the closest approach, there is $2 \mathrm{~mm}$ of space between the cylinder and the wall enclosing the sensors, and any such complications should be present. On the other hand, at large distances 


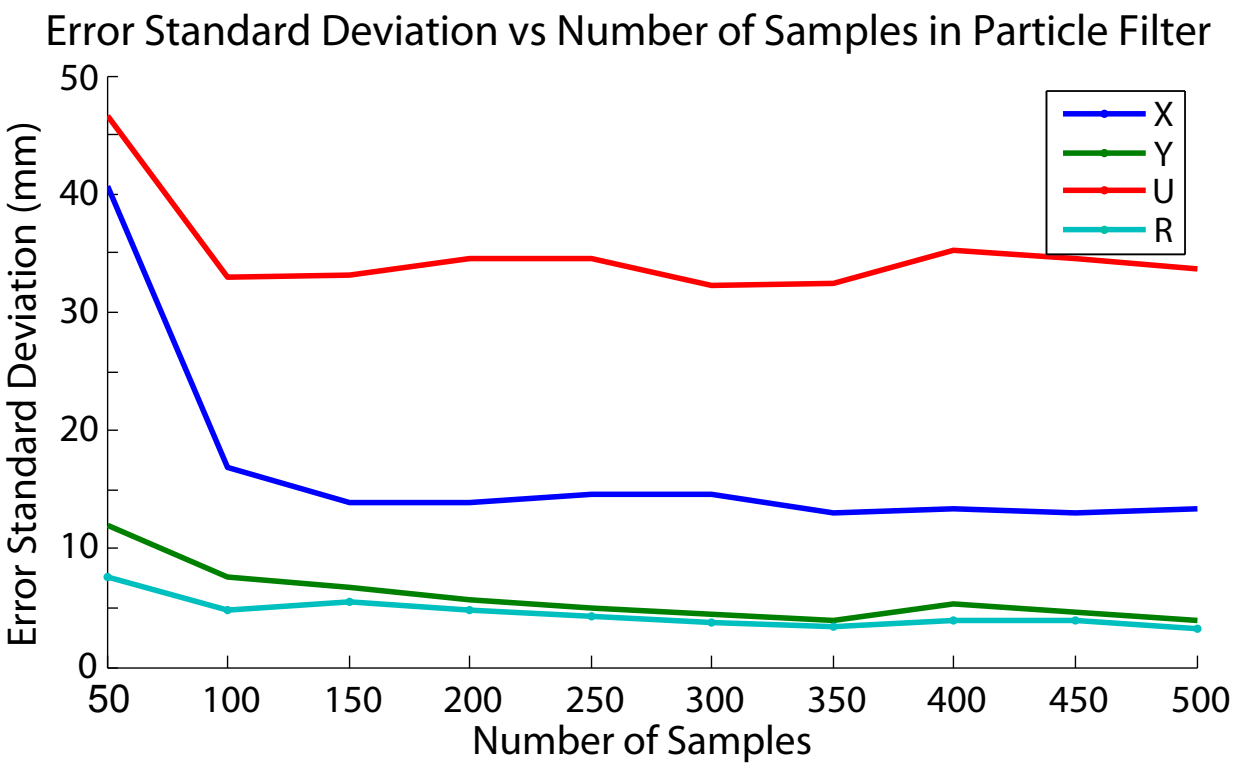

Figure 3-17: The dependence of error standard deviation on particle number. The standard deviation in the average error, calculated over the same period of time as for Figure 3-16. The error standard deviation reaches a steady state with respect to the number of particles earlier than the error bias.

between the cylinder and the sensors, the pressure field from the cylinder is reduced in magnitude and spread out in space. This makes it more susceptible to contamination from other noise sources. It appears from the experimental results that the error in the x position is affected by the increasing distance, but not by close proximity to the sensors. Both the y position and the velocity cannot clearly be connected to either relationship based on the experimental data. In the case of both of these variables, it is unclear why there would be increased error in the middle distances. It is possibly a result of the limited number of experimental trials. For the radius, both the increasing error with increasing distance and the increased error at very small distances may be present. There are signs of the radius estimate consistently degrading at the closest two distances. There are also suggestive signs of the error increasing again at larger distances, though further experiments at larger distances would be needed. At some point, the error in all the variables will increase as the distance passes some threshold, since signals measured by the sensors will fall below the noise level. However, at the intermediate distances examined here, this affect does not appear to be significant except for the x position. 


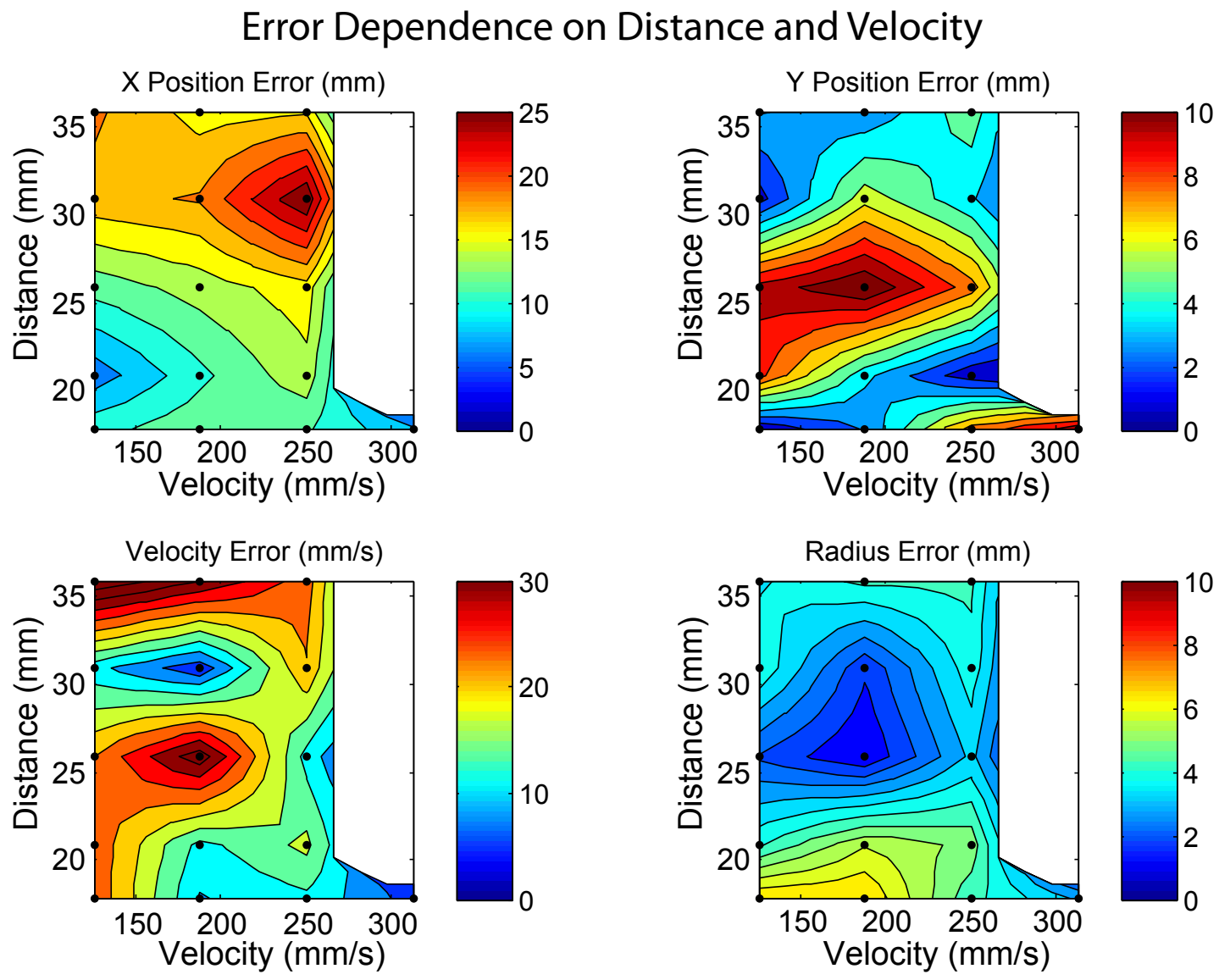

Figure 3-18: Particle filter error landscape. The dependence of each state variable estimate against the speed and distance of a moving cylinder using a particle filter based on a Rankine half-body model is shown. Each point corresponds to the averaged absolute error based on five experimental runs.

\subsection{Discussion}

The results of the particle filter in estimating the position and size of a moving cylinder demonstrate that the state of a moving round cylinder can be tracked in real time using a fix analytic model as the base. They also provide the framework for accomplishing the cylinder tracking in real time. The error in the state estimation follow some predictable relationships with the speed and distance, but not all variables are affected equally.

The model was chosen to capture the dominant characteristics in the pressure response of a moving round cylinder and its wake as predicted by computational fluid dynamics. The simple potential flow model of a Rankine half-body, generated by a source in a steady 
uniform flow, matches the shape and amplitude of the pressure distribution over a line parallel to the flow (and thus motion of the cylinder). In addition, the transition from high to low pressure is correctly located and reproduced. The model deviates slightly the decay rate in front of the peak pressure, decaying to slowly. In the wake region, the model roughly captures the mean pressure recovery, but the unsteady nature of the wake means that there are significant differences between the model and any instantaneous pressure distribution.

Based on the relationship between the state variables of interest, and an analysis of the Kalman filter and particle filter estimation results, approaches that rely only on Gaussian noise models are unsuitable for tracking a moving cylinder. A method without a priori noise parameterization, such a particle filter, is better suited for tracking a cylinder size and position. The Kalman filter implementations were only successful in tracking a cylinder from simulated data with accurate initial states and were unable to track a cylinder from experimental results, whereas the particle filter was able to accurately track cylinders based on experimental data. The failure mechanisms in tracking the cylinder of the Kalman filter, such as running off a boundary, are consistent with the use of a Gaussian model for nonGaussian noise.

A particle filter has been used to track the cylinder position and size given a history of pressure measurements. Based on the analysis of the number of particles needed for the reliable behavior of the particle filter in this application, as low as 200 particles could be used with a reasonable expectation of consistency. At that level (200-300 particles) a sampling rate of $20 \mathrm{~Hz}$ is needed for the particle filter to run in real time in a MATLAB implementation on a $2.66 \mathrm{GHz}$ laptop. This speed could likely be improved. However, 20 $\mathrm{Hz}$ is sufficient to capture the relevant features of the pressure signals and is sufficiently fast for changes in the state that might be expected if the y position or velocity shifts.

By applying the particle filter based on the Rankine model to the full test matrix of experimental data, it is possible to get a sense of how reliable the cylinder tracking estimate is, and how it depends on the distance and speed of the moving cylinder. The expectation was that the error in tracking a cylinder increases slowly with increasing distance and decreasing velocity. The error was also expected to increase rapidly below some lower limit in distance. Based on the results from the experimental data, the error in tracking was found 
to be largely independent of velocity for the ranges tested, with the possible exception of the $\mathrm{x}$ position at high velocity. Though not large, there does appear to be a correlation between the error in radius and in the $\mathrm{x}$ position against distance.

The error dependence on distance and velocity can be considered from the perspective of collision avoidance and navigation in order to evaluate whether the approach outlined in this chapter would be adequate for application to AUVs operating in difficult environments. The experiments of this chapter involved a moving cylinder near stationary sensors, but the results would be expected to be very similar for a stationary object in an external flow, with a moving AUV sensor platform. In this case, there would be some differences in the unsteady component of the wake, but outside the envelope of the separated flow this change of reference frame can be applied with minimal discrepancies. Of course, any pressure distribution about the AUV would have to be taken into account.

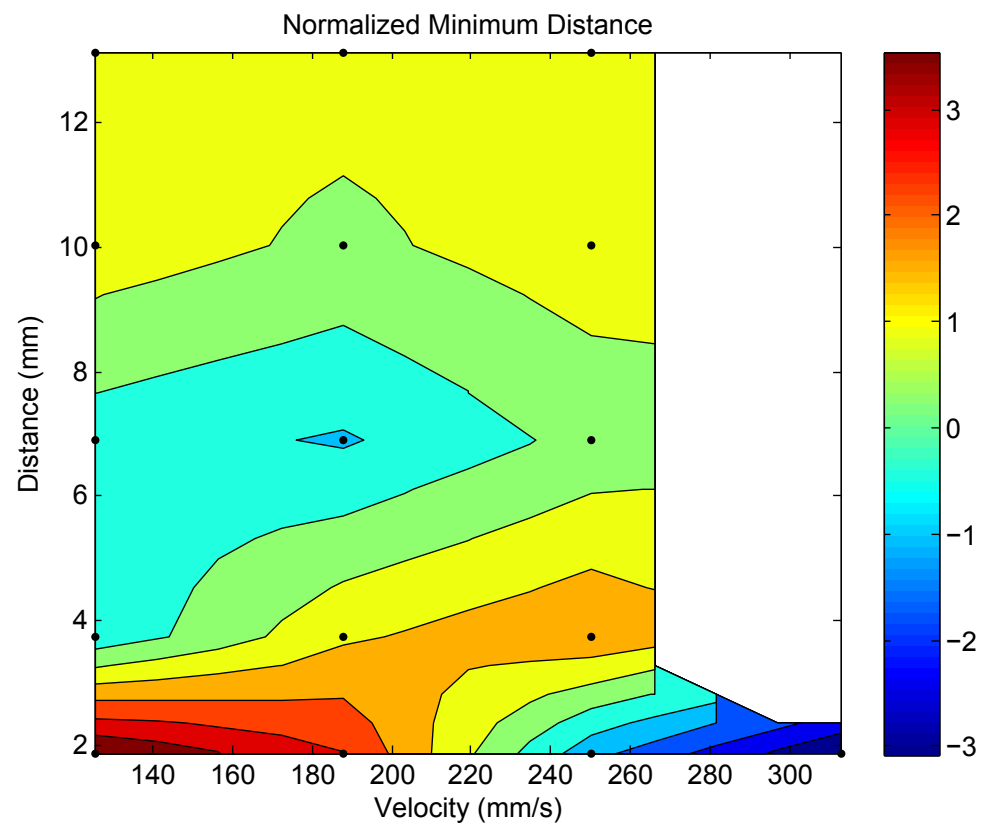

Figure 3-19: The perpendicular distance between the estimated cylinder position and the sensor array. The estimated distance is normalized by the actual distance between the closest point of the cylinder and the sensor array line.

Based on the overall observations, the cylinder tracking estimates tend to be relatively consistent, but with bias in the estimation of some of the variables under certain conditions. The most substantial of these is the tendency to underestimate the distance between the 
sensors and the cylinder. However, at very small distances, the radius of the cylinder was also substantially underestimated. In terms of the position along the sensor array, the error increased with velocity at large distances. From the point of view of collision avoidance and navigation however, the minimum distance between the object being tracked and the sensor array is the primary concern. If this minimum distance is underestimated, then it implies that an AUV will be more conservative in maneuvering near the object. If the distance is overestimated, the vehicle may collide with the cylinder. Clearly it is preferable to err on the side of underestimation. The results based on the $y$ and $r$ estimates from the experimental matrix are shown in Figure 3-19 with the estimated minimum distance normalized by the true minimum distance. This implies that a value less than 1 corresponds to an estimate that reduces the likelihood of collision, whereas a value greater than one implies that the estimate creates a risk of collision. A value of zero or below implies that the estimate indicates a collision is occurring in the simulation. In examining the figure, it is evident that the collision distance is only overestimated for the closest distances in which the separation is $2 \mathrm{~mm}$, which is due to the underestimation of the radius that was remarked upon earlier. With the distance so small, the reduced radius estimate results in an error that could lead to real collisions. However, practically speaking, this separation distance between the cylinder and array of $2 \mathrm{~mm}$ is already effectively a collision, since maneuvering with that degree of precision is difficult in unpredictable environments. At slightly larger experimental distances, the separation underestimated (with a few false collisions where the $\mathrm{y}$ position is most severely underestimated). These errors are not as significant as overestimating the distance, since they lead to more conservative navigation but do not result in collisions. Beyond the intermediate distances, it appears that the estimate of the separation settles to a reasonable approximation of the true separation. The results therefore are promising, indicating that for all but the closest distances, a lateral-line-like pressure sensor array could be used for object avoidance in environments with currents. 


\section{Chapter 4}

\section{Object Identification and Classification}

\subsection{Introduction}

This chapter addresses the ability pressure sensor arrays, emulating the lateral line organ, to distinguish the shapes of physical objects when they move within their stimulus range. While the workings of the lateral line organ itself are progressively better understood, there is still comparatively little known about the information extracted from the lateral line by the fish, outside of that corresponding to the dipole stimulus.

As discussed in Chapter 2, hydrodynamic stimuli from aquatic animals other than small prey has received the least attention in biological studies. Larger fish, potentially predatory, are not well modeled by small oscillatory dipoles and instead would be better approximated by blunt moving objects. Experiments by Vogel and Bleckmann [80] have demonstrated that goldfish use their lateral line to respond to changes in size, velocity and shape of passing rods in still water. The results of Chapter 3 have shown that there is sufficient information to track the size and position of a passing rod, but did not address the question of shape.

Two studies ([70] and [6]) have considered parameterizations of the shape of an arbitrary object suitable for modeling flow and pressure distributions in potential flow. The authors have used these parameterizations to demonstrate how a simulated fish swimming in a potential flow could identify the shape of an object by swimming past or around it. However, these models are not able to take into account the wake that forms behind a 
moving object which is a significant factor in lateral line measurements when the object is moving. Despite the simulation results estimating the shape of an object in potential flow, it is unclear how much of the shape information is altered when a wake is present.

Through the experiments presented in this chapter, we investigate the information concerning the shape of non-oscillatory stimuli that can be obtained through a pressure sensor array. As a representative problem, the study concentrates on whether it is possible to distinguish between cylinders with square and round cross-sections. These stimuli are similar in basic structure and size, but the presence of sharp corners and the bluntness of the front face may distinguish the shape of the square. Similar to the earlier behavioral experiments by Vogel and Bleckmann [80], the objects are moved past stationary sensors to generate the signals.

After a description of the relevant experiments, the chapter first examines the average response of a lateral-line-like sensor to a passing cylinder of different shapes. Individual pressure traces generated by the time history of a single sensor as a cylinder passes by will vary greatly from one trial to the next due to the wake of the cylinder. By inspecting the averaged results, differences in the pressure response due to shape will be revealed, leading to features that can be used to distinguish the shape of a cylinder based on a single pass. This approach succeeds in distinguishing the cylinder shapes, but as will be shown, it is limited since the features are connected to only a few points in the pressure response. A second approach for feature extraction is subsequently discussed and evaluated, using a principal component analysis to generate a single decision criterion that incorporates the entire pressure response. This is much more successful, but requires interpretation in order to understand what it may be using as a basis of the decision.

\subsection{Experimental Procedure}

The experiments described in this chapter are similar to those of the previous chapter in that both are focused on a vertical cylinder passing by a stationary horizontal linear pressure sensor array in still water. However, whereas the previous chapter focused on demonstrating that the size and position of a moving cylinder could be tracked in real time, the 
presenta chapter is concerned with extracting the shape of the moving cylinder based on the near-field pressure. As a result of the change in experimental focus, there are substantial differences in the experimental setup. Unlike the experiments from Chapter 3, the experiments in this chapter are designed with a heavier emphasis on repeated measurements. This is accomplished at the cost of reduced precision in maintaining some of the free experimental parameters constant. The experiments completed in the Singapore towing tank allowed the very precise placement and motion of the cylinder, but, due largely to the small dimensions of the tank, standing waves would develop after a single experimental pass that would influence the measurements. For this reason, large numbers of trials were impractical. In contrast, the large dimensions of the MIT towing tank coupled with the artificial beach at one end significantly dampen surface waves and therefore allow for repeated trials with minimal delays. Unfortunately, the positioning of the cylinder is less precise due to the large distance between the carriage and the test section (approximately 1.5 vertical meters) and to difficulties in the precise placement of the sensors.

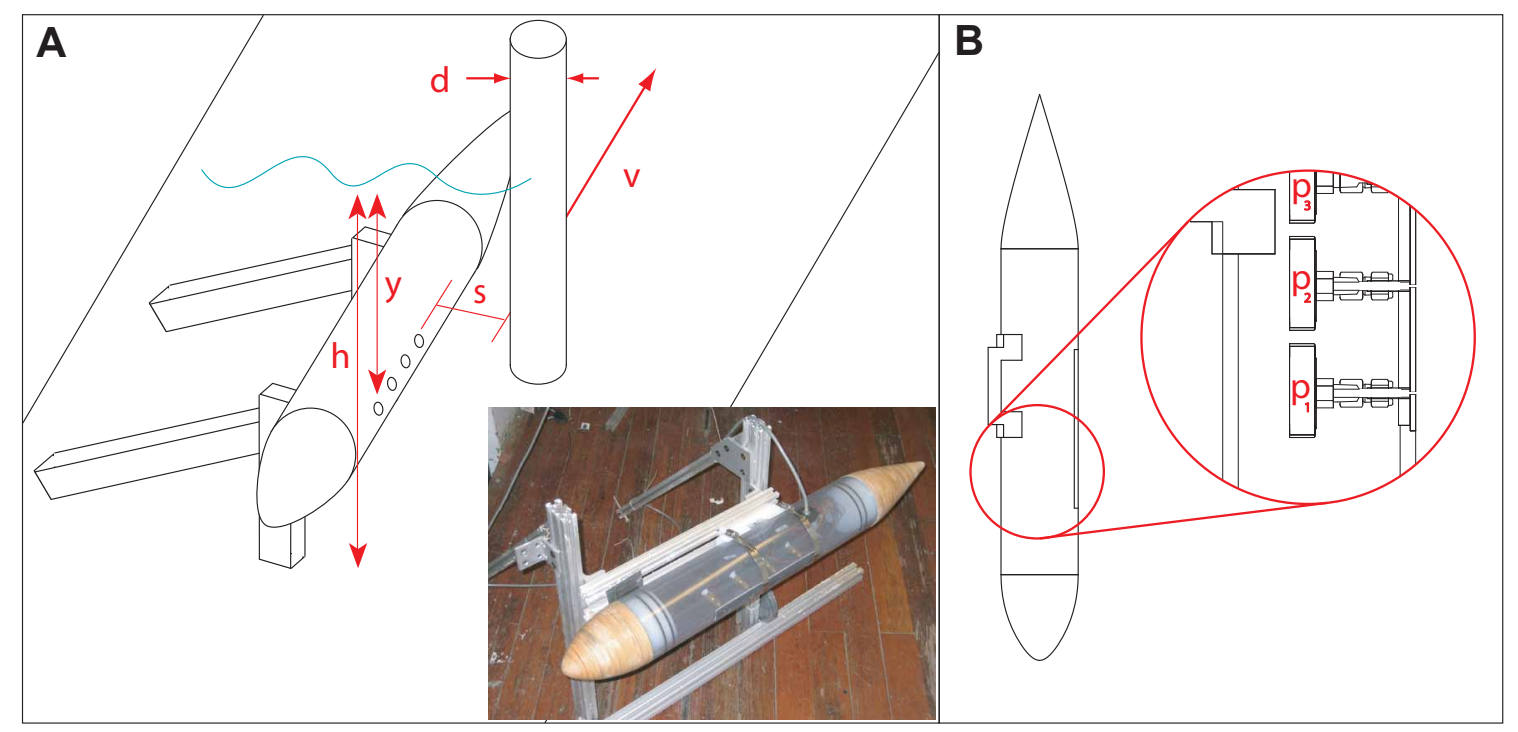

Figure 4-1: A: Schematic of experiments with cylindrical stimuli. The diagram depicts the housing with pressure ports along the side and a typical cylinder moving alongside. Several key quantities are labeled, including the separation between sensors and cylinder (s), the depth of the sensors (y), the total depth of the tank (h), the diameter of the cylinder (d), and the velocity of the cylinder (v). B: A close up of the pressure sensor and port geometry. The pressure sensors are labeled $p_{1}-p_{3}$ in the cut-out view. 


\subsubsection{Experimental Layout}

The fundamental aspects of the layout of the experiments described in this chapter are the same as in Chapter 3. A vertical cylinder was passed in front of an array of stationary pressure sensors, much in the same manner. The experiments were performed in the MIT Towing Tank, where the water tank has dimensions $40 \mathrm{~m}$ by $2.5 \mathrm{~m}$ by $1.3 \mathrm{~m}$ equipped with an overhead carriage capable of towing at speeds up to $1.5 \mathrm{~m} / \mathrm{s}$. The housing containing the sensors was fixed near the center of the tank and aligned with the motion of a movable carriage. Vertical cylinders attached to the carriage were towed at constant velocity past the sensors. The specific configuration can be seen in Figure 4-1A. The motion of the cylinders began five meters upstream from the sensors, reached its final velocity within 0.5 meters from the start, and continued up to two meters past the sensors. Six seconds of pressure data were recorded at $2 \mathrm{kHz}$.

Besides the tank size, the housing that contained the pressure difference was significantly different than the one used for the cylinder tracking experiments. The sensor housing for the cylinder shape detection was based on a cylindrical body with a diameter of 11.4 $\mathrm{cm}$ (4.5 in), and a streamlined nose and tail (Figure 4-1). The sensors are ported to the side of the cylinder, aligned with the main cylinder axis.

The pressure sensors used in the experiments were gauge pressure sensors made by Honeywell, part 242PC15M, with a nominal sensitivity of $48 \mu \mathrm{V} / \mathrm{Pa}$ and response time of $1 \mu \mathrm{s}$. These sensors included internal amplification, which meant they could not be grouped closely in an array. The four sensors were enclosed in a streamlined housing as shown in Figure 4-1 and were connected to the outside flow through ports spaced $6.35 \mathrm{~cm}$ (2.5 in) apart. The sensor port geometry can be seen in Figure 4-1B. The density of this linear array was limited by the size of the pressure sensors. However, since the stimuli in the experiments were moving at constant velocity, the time series from each sensor can be used to approximate the spatial response sampled at intervals $d=V / f_{s}$, where $\mathrm{V}$ is the velocity of the stimulus and $f_{s}$ is the sampling frequency. This is an accurate approximation wherever the flow is steady in the frame of the moving stimulus, but does not hold as well in the wake region of the cylinders, where the distribution and location of vortices is time 
dependent. The sensors were connected to a rigid tube with a $0.472 \mathrm{~cm}(0.186$ in) inner diameter. A small hole $1.3 \mathrm{~mm}$ (.050 in) in diameter and $3.2 \mathrm{~mm}(0.13 \mathrm{in})$ deep connected the tube to the outside fluid. This layout was chosen to minimize the noise, given that it was not possible to mount the pressure sensors flush.

In order to minimize any additional electrical noise picked up through interference, the signals were further amplified using a similar circuit as in Chapter 3 (Figure 4-2. Since the sensors used in these experiments already have some internal amplification, a gain of 10 was used. A single voltage divider with a potentiometer was used to remove the common zero offset generated by the static pressure from all of the sensors. This was necessary in order to avoid the saturation of the data acquisition system (NI USB-6210). Due to the comparative sizes of the amplification circuitry and the sensor housing, the amplification in these experiments occurred outside of the water, connected by a foil-shielded cable. As with the other experiments, the data acquisition was coupled with an unconnected laptop in order to minimize electrical noise from building electrical sources.

\subsubsection{Test Matrix}

The difference between square and circular cylinder cross sections is of primary interest, but it is also important to test the dependence of the results on the towing speed, the cylinder diameter, and the distance from the cylinder to the sensors. This is necessary in order to have some confidence that the results demonstrate that different shapes are being distinguished, instead of different particular experiments. In all of the experiments, the orientation of the square cross section was kept constant with the leading face perpendicular to the oncoming flow. Two sizes for each cross section were tested: $5.08 \mathrm{~cm}(2 \mathrm{in})$ and 7.62 $\mathrm{cm}$ (3 in). For the round cross section, size refers to the diameter, and for the square cross sections it refers to the side length.

Two carriage velocities were also tested at $0.50 \mathrm{~m} / \mathrm{s}$ and $0.75 \mathrm{~m} / \mathrm{s}$. Most of the tests were performed at a separation of $0.51 \mathrm{~cm}(0.2 \mathrm{in})$, but two sets were also done at a larger separation of $1.27 \mathrm{~cm}(0.5 \mathrm{in})$. A total of ten sets of experiments were run with different combinations of these variables as shown in (Table 4.1). Each set consisted of one hundred 


\begin{tabular}{|l|c|c|c|c|}
\hline \multirow{2}{*}{} & \multicolumn{4}{|c|}{$5.1 \mathrm{~mm}$ Separation } \\
\cline { 2 - 5 } & \multicolumn{2}{|c|}{$7.62 \mathrm{~cm}$ Diameter } & \multicolumn{2}{|c|}{$5.08 \mathrm{~cm}$ Diameter } \\
\cline { 2 - 5 } & $0.50 \mathrm{~m} / \mathrm{s}$ & $0.75 \mathrm{~m} / \mathrm{s}$ & $0.50 \mathrm{~m} / \mathrm{s}$ & $0.75 \mathrm{~m} / \mathrm{s}$ \\
\hline Square & 100 Runs & 100 Runs & 100 Runs & 100 Runs \\
\hline Round & 100 Runs & 100 Runs & 100 Runs & 100 Runs \\
\hline
\end{tabular}

Table 4.1: Table of tests performed for identifying a moving object. Not included in the table are two sets of 100 runs (one for each cross section shape) at $0.75 \mathrm{~m} / \mathrm{s}, 5.08 \mathrm{~cm}$ diameter, and $1.27 \mathrm{~cm}$ separation.

passes of the cylinder past the sensors. These experiments correspond to Reynolds numbers between $2 \cdot 10^{4}$ to $6 \cdot 10^{4}$ and to ratios of the separation between sensors and object (labeled $\mathrm{s}$ in Figure 4-1) to object size between 0.1 and 0.3 . These conditions and the geometric arrangements were chosen in order to be relevant to the situation of a fish exploring a new environment (see [82]), and to a vehicle operating within a cluttered environment.

\subsubsection{Data Pre-Processing}

Calibration of the sensors was done immediately after testing without removing the setup from the water. In order to calibrate all of the sensors at the same time and in situ, a wave machine was used to generate waves at approximately $1.5 \mathrm{~Hz}$. The amplitude of the waves was measured above the position of the sensors at the same time as the pressure oscillations from the waves were recorded. The calibration was calculated using linearized surface wave theory [60], in which

$$
A_{p}=\rho g \frac{\cosh [k(y+h)]}{\cosh [k h]} \cdot A_{w}
$$

determines the amplitude $A_{p}$ of the pressure oscillation a distance $y$ below the surface in water of depth $h$ when the wave amplitude $A_{w}$ is measured. In the equation, $\rho$ is the density of water, $g$ is the gravitational acceleration, and $k$ is the wave number. The wavenumber of the surface waves is calculated from the dispersion relation $w^{2}=g k \tanh [k h]$, where the frequency $w$ was calculated by fitting a sine to the recorded pressure.

The Honeywell sensors used in these experiments have a substantial amount of white noise in their measurements. In tests using a manometer to apply a constant measureable 

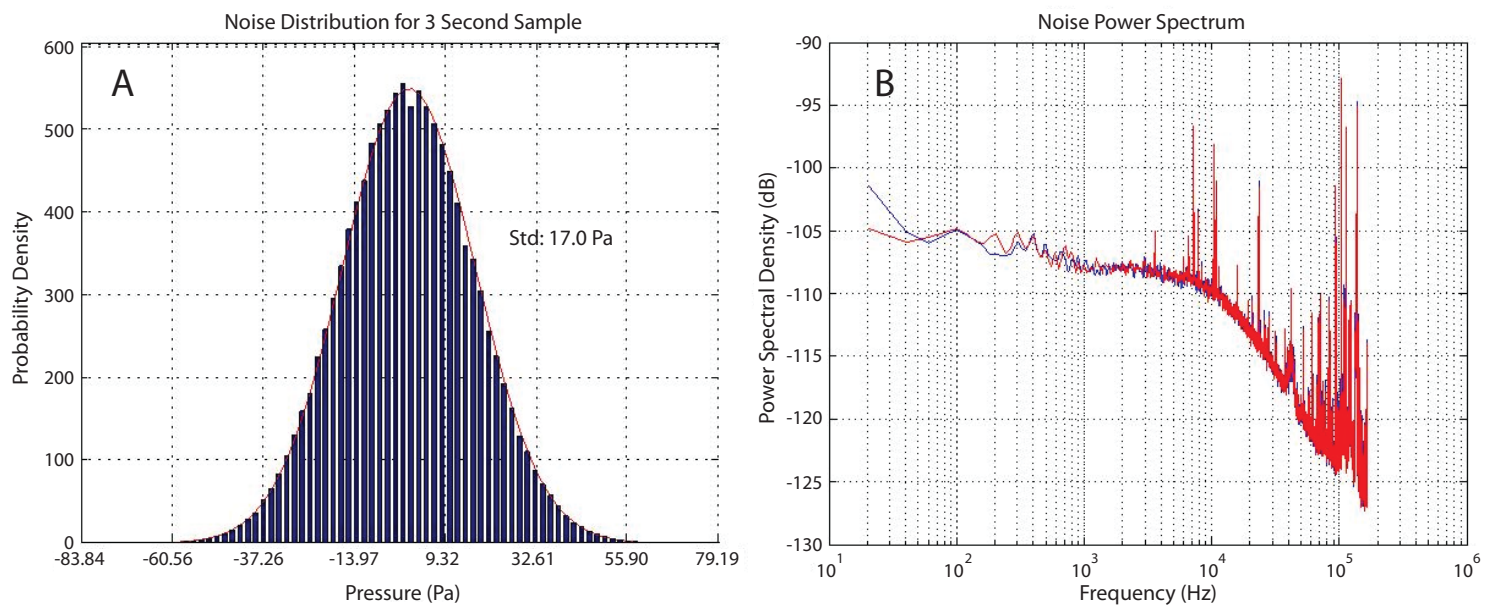

Figure 4-2: A: Resting noise distribution of the Honeywell 242PC15M pressure sensor. the normalized histogram is generated from a 3 second sample at $300 \mathrm{kHz}$. B: The corresponding power spectrum to the noise sample. High frequency electrical noise peaks are evident in the power spectrum.

pressure, it was found that the sensor noise had a standard deviation of approximately 17 $\mathrm{Pa}$, based on a measured sensitivity of $42 \frac{\mu \mathrm{V}}{\mathrm{Pa}}$ which is below the specified $48 \frac{\mu \mathrm{V}}{\mathrm{Pa}}$. Figure 4-2 shows the clearly Gaussian noise distribution, as well as the corresponding power spectrum which does not contain much notable electrical interference until very high frequencies. These tests demonstrate the character of the sensors only, as the tests did not include the additional external amplification and utilized very short (less than a foot) cabling.

Since the experiments were done in a much larger tank which was not susceptible to establishing long wavelength standing waves, it is unnecessary to consider the difference between pressure sensors in this Chapter, unlike Chapter 3. Instead, the raw data were processed as follows: First the hydrostatic pressure, taken as the mean pressure before the cylinder approached (where the pressure signal is flat), was subtracted from the data of each sensor for every run; all pressures shown in the figures and discussed in the text are pressure differences with respect to the hydrostatic pressure. The cylinder stimuli were run at two velocities $(0.5 \mathrm{~m} / \mathrm{s}$ and $0.75 \mathrm{~m} / \mathrm{s})$ past the sensors, but the data was collected at $2 \mathrm{kHz}$ in all cases; this is equivalent to sampling the slow tests more closely in space. For this reason, the data from slower velocity runs were then downsampled by a factor of $3 / 2$, making them comparable to the higher speed data. Following this, each pressure trace 
was aligned using the point at which pressure crosses zero (with negative slope), near the leading edge of the stimulus. As can be seen from Figure 4-3, this feature is prominent and easy to identify: the steep slope at that point minimizes the variance due to noise. This point of alignment does not correspond to the same physical location of the stimulus for all cases. Experiments using an external trigger showed that the zero crossing occurs slightly earlier for square cross sections than for the round cross sections. However, considering the actual use of a lateral line, the position of a moving object would not be available, and hence the zero crossing makes a very good alternative to use. In general, the data were not filtered before further processing, despite the large noise associated with the pressure measurements.

The result of the data processing is a large set of pressure traces corresponding to each pressure sensors. Each of these traces is aligned and sampled at the same spatial intervals, with the same pressure reference point. This allows a direct comparison and averaging between runs with very different parameters, such as that needed to generate Figure 4-3.

\subsection{Results}

The pressure traces all had a set of basic similarities. Figure 4-3 gives the mean pressure traces for both cylinder cross sections with two inch diameters and at $0.75 \mathrm{~m} / \mathrm{s}$ which illustrates the typical response. In all cases, the pressure response to a passing cylinder could be described as a slow, near-exponential rise in pressure ahead of the cylinder, followed by a sharp transition to low pressure and a recovery back to the hydrostatic pressure. Due to the nature of the viscous flow around the cylinder, there is a large variance between runs under the same conditions. This variance is not evenly distributed across the entire experimental run, however. Figure 4-3 depicts the standard deviation; for each run there is little deviation from the mean value of 100 runs until the point of sharp transition to low pressure is reached. From that point on, there is a significant variation which decays slowly with time. This variance stems from the turbulent motions in the wake and especially the presence of vortices, which manifest themselves as isolated low-pressure peaks (Figure 4-4). Although vortices are shed regularly at the Strouhal frequency, the wake is turbulent, disturbing the 


\section{Comparison of Mean Response Due to 2" Cross Sections at $0.75 \mathrm{~m} / \mathrm{s}$}

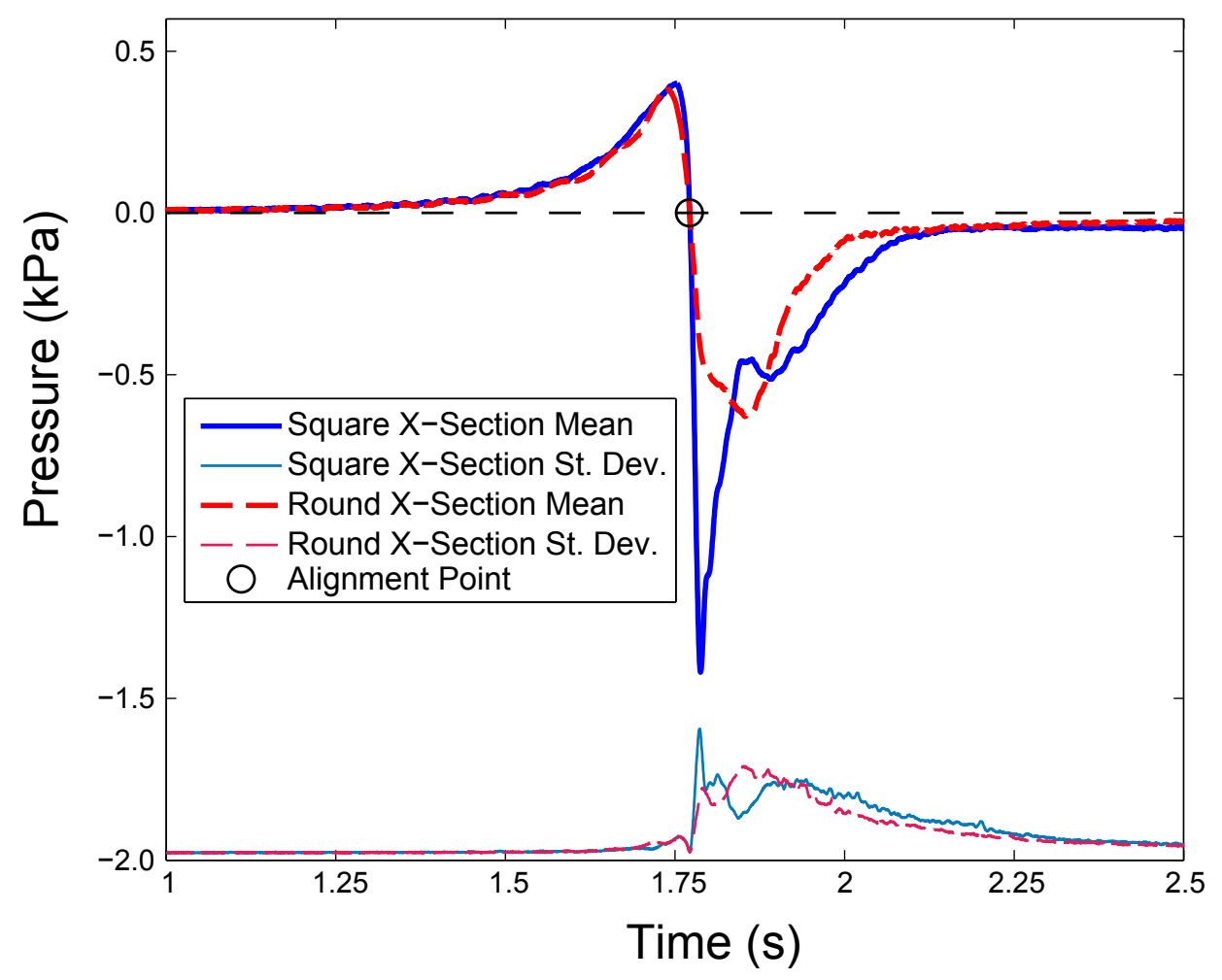

Figure 4-3: Results from two sets of tests comparing pressure traces obtained from two different cross sections. Both cross sections had a diameter of two inches, velocity of 0.75 $\mathrm{m} / \mathrm{s}$, and separation of $0.51 \mathrm{~cm}(0.2 \mathrm{in})$. The mean value of all four sensors in each set of 100 runs is shown. The corresponding standard deviation is offset by $-2 \mathrm{kPa}$, but on the same scale as the mean pressure traces.

regularity in the position of the vortices; hence pressure measurements contain random noise. The phase of vortex shedding determines whether a vortex is shed close to one of the stationary sensors.

The mean value of the pressure traces as function of time, obtained for different size, velocity, and shape, are shown in Figures 4-5 to 4-7. In each case, the mean pressure trace is found across all sensors.

In comparing the results from the differently sized cylinders (Figure 4-5), we find that there is little change in the overall pressure amplitude. There is, however, a consistent increase in the time interval between the zero crossing at the leading edge and the time 


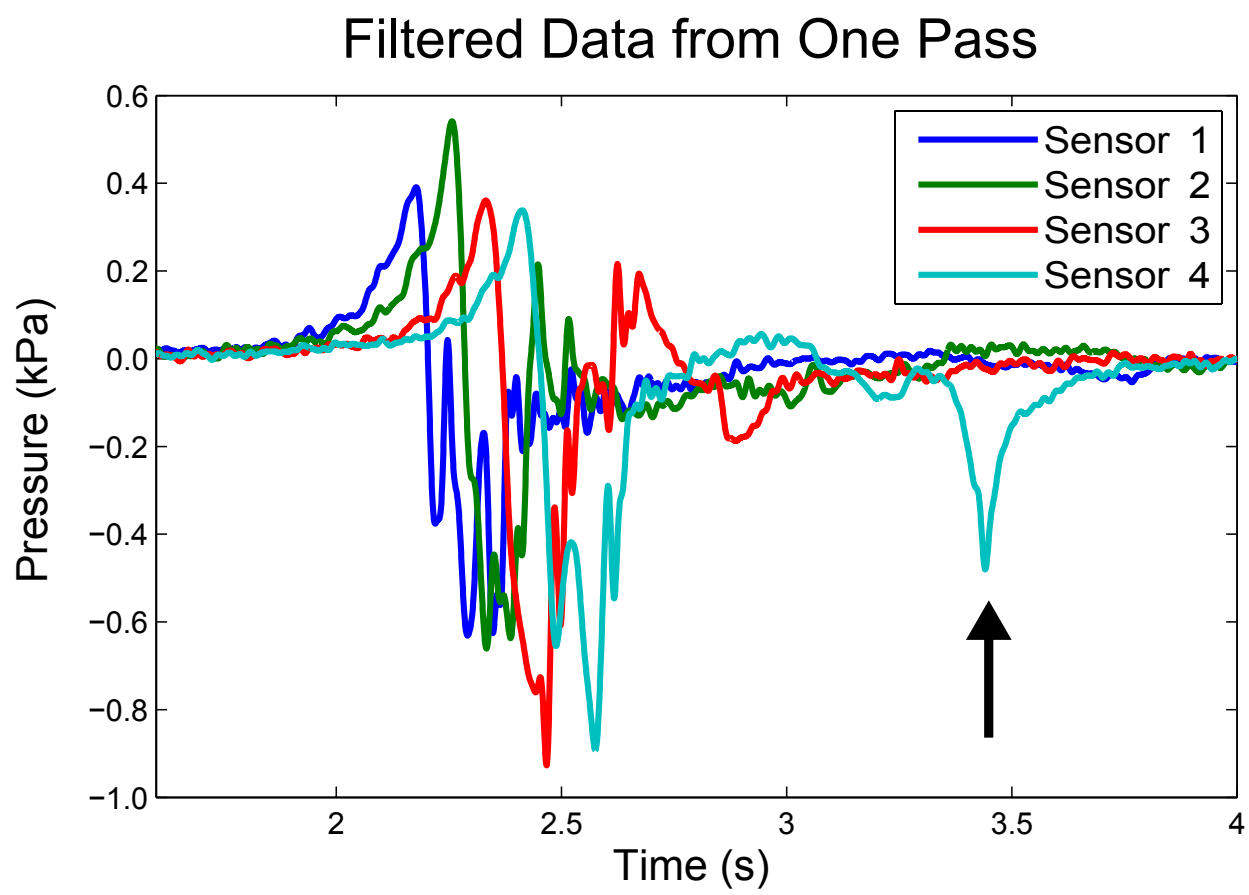

Figure 4-4: Pressure traces for a single run with a round cylinder are depicted, showing the variability accross measurements from individual sensors. These traces were filtered with a cut-off frequency of $100 \mathrm{~Hz}$ but not aligned. The low pressure spike marked with an arrow has the signature of a vortex impinging on the sensor port. 


\section{Size Comparison of Mean Pressure Traces}
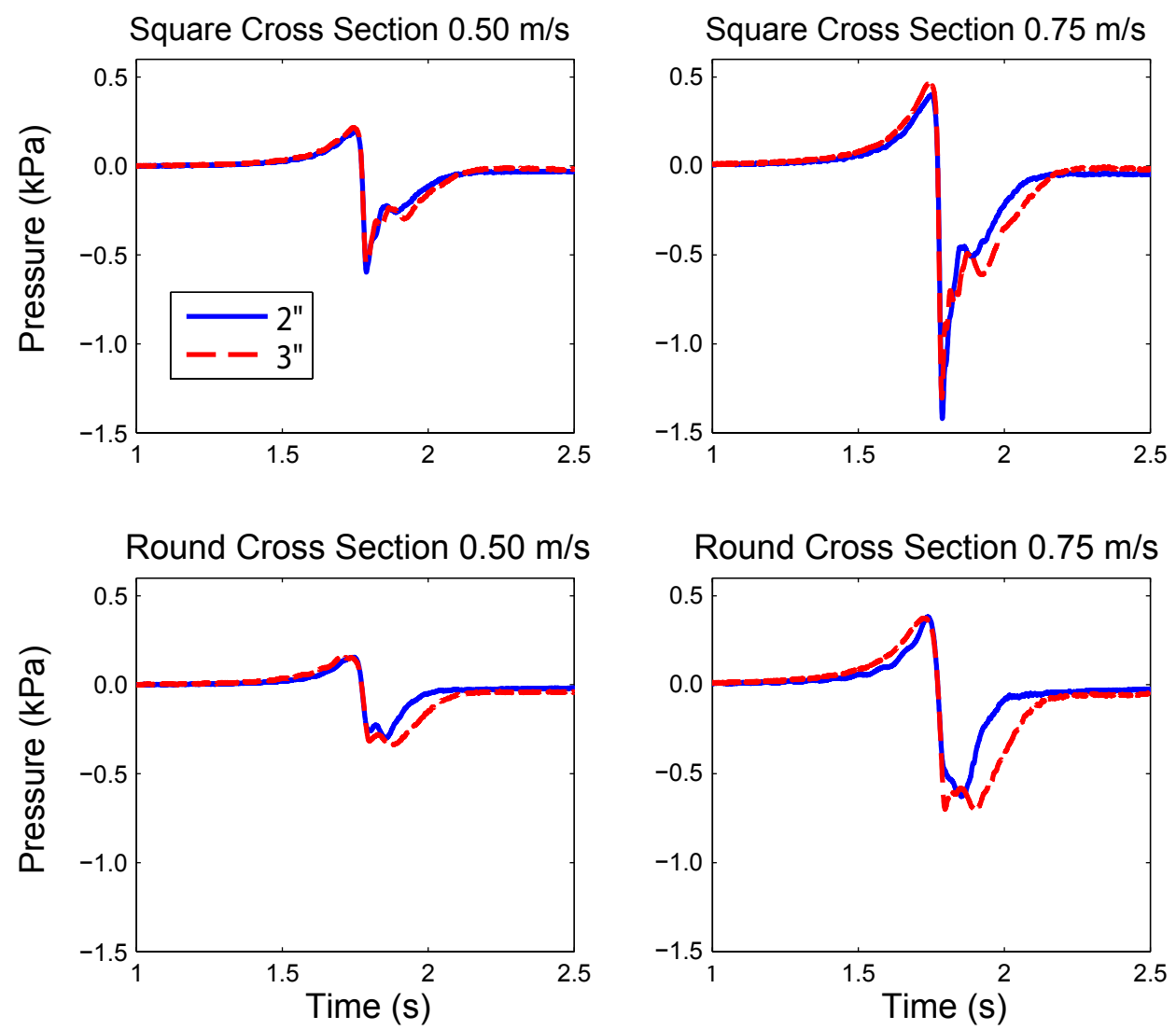

Figure 4-5: The mean value of the pressure as function of time. Eight data sets are presented, cross-compared to assess the effect of cylinder size. Each data set is composed of 100 runs. 


\section{Velocity Comparison of Mean Pressure Traces}
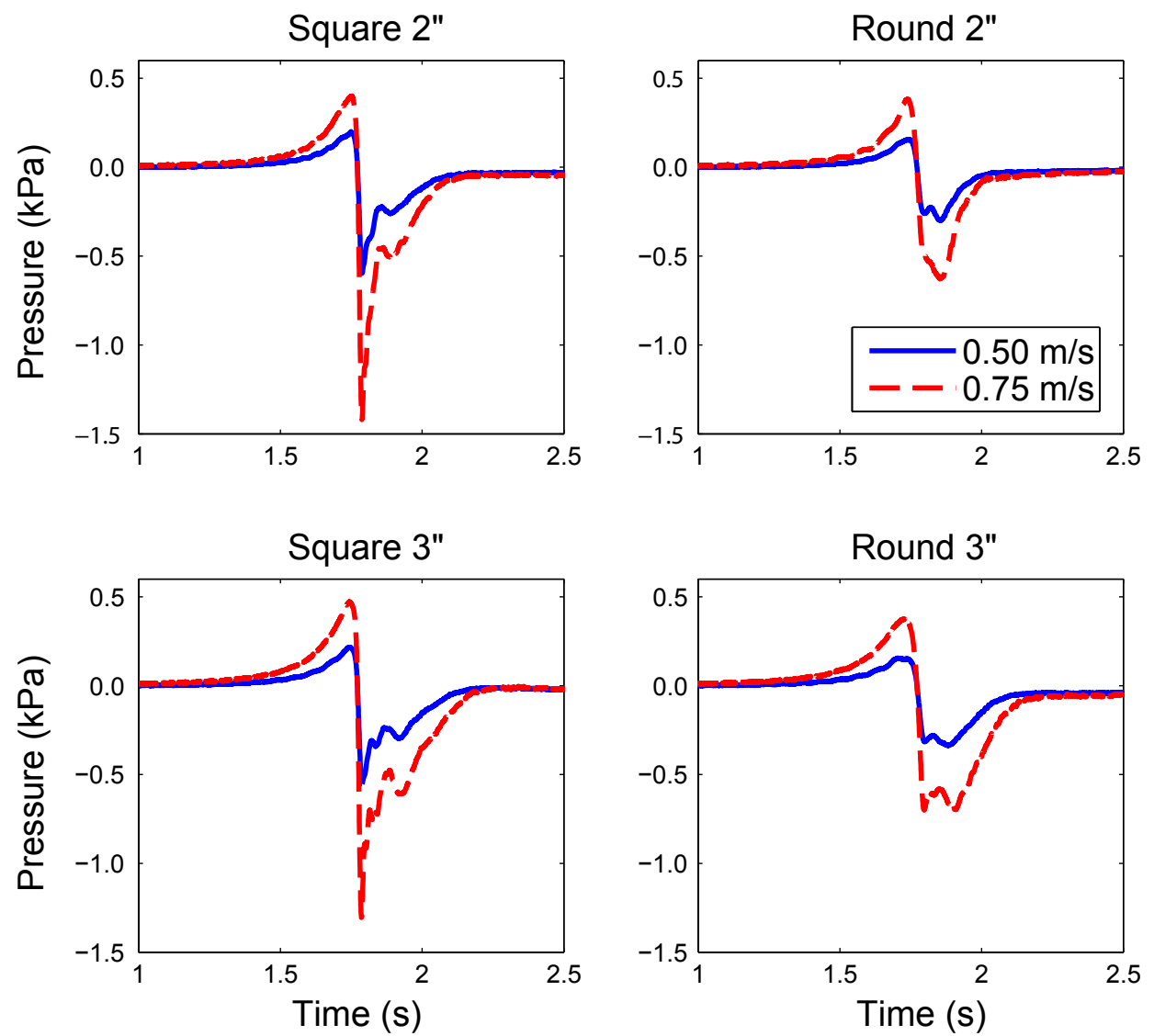

Figure 4-6: The mean value of the pressure as function of time. Eight data sets are presented, cross-compared to assess the effect of cylinder velocity. Each data set is composed of 100 runs. 


\section{Comparison of Cross Sections in Mean Pressure Traces}
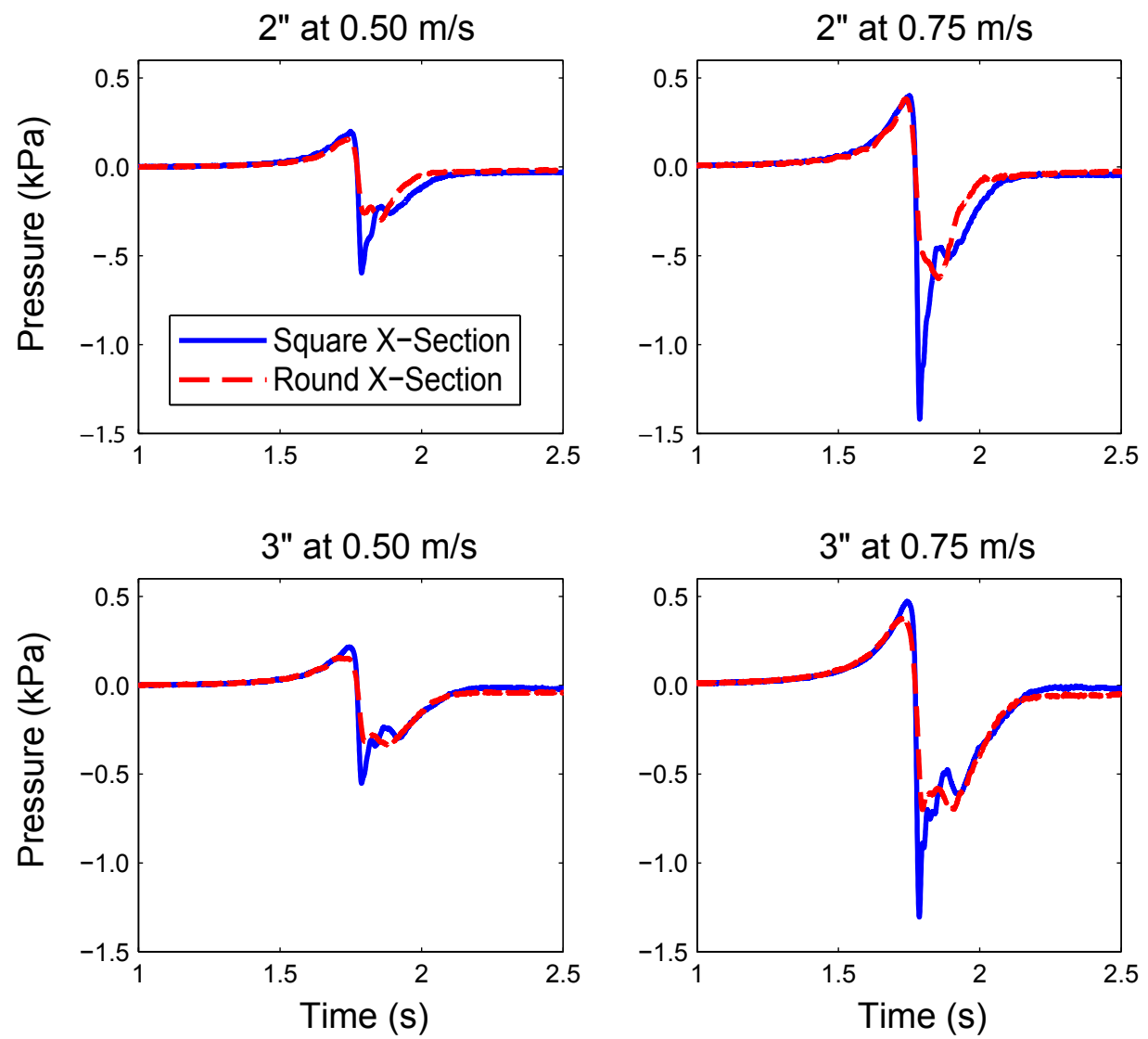

Figure 4-7: The mean value of the pressure as function of time. Eight data sets are presented, cross-compared to assess the effect of cylinder shape. Each data set is composed of 100 runs. 
when the pressure returns to the hydrostatic pressure value. While this change is evident in the mean pressure traces, it is much more difficult to pick out in the individual pressure responses due to the large flow variability in that region, which corresponds to the wake region.

A cylinder moving at a higher velocity creates a larger amplitude pressure response. Recall that due to the resampling of the lower speed data, this is an effective comparison of the spatial pressure fields and not the time histories. The increased amplitude is evident in Figure 4-6 and is expected from potential flow. Indeed, a potential flow model of a circular cylinder in steady flow predicts that the pressure is given as:

$$
P-P_{\infty}=U^{2} \cdot C(\bar{x}, r, \rho)
$$

where $U$ is the velocity of the cylinder, $\bar{x}$ is the position vector from the center of the cylinder, $r$ is the radius of the cylinder, and $\rho$ is the density of water (refer to [60]). The model is not quantitatively accurate because it is based on a round cross section and cannot take into account the wake region; however, it provides qualitatively the correct velocity dependence. For example, the model predicts that the pressure at $0.75 \mathrm{~m} / \mathrm{s}$ should be $\frac{9}{4}$ (2.25) times larger than the pressure trace at $0.50 \mathrm{~m} / \mathrm{s}$. The ratios found by fitting least squares in the experimental traces were found to be $2.09,2.12,2.13$, and 2.15, in clockwise order, according to Figure 4-6. There does not seem to be any systematic dependence on the shape or size of the cylinder on this quantity. The scaling effect of velocity is particularly convenient because it is possible to accurately determine the velocity of the stimulus by comparing the time lag between sensors. The effects of velocity can, therefore, be immediately removed from further analysis of the shape of the cylinder.

Finally, the results from different cylinder cross-sectional shapes are compared in Figure 4-7. Qualitatively, there are a number of apparent differences between the mean traces. The most striking difference is that the square cross section is characterized by a sharp peak of low pressure following immediately after the zero cross point. This is true for all sizes and velocities tested. On the other hand, other differences depend on the size of the cylinder. In particular, there is a wake length difference for the smaller cylinders that is not 
present for the larger cylinders. Also, the initial peak, before the zero crossing point, seems larger and sharper for the square cross sections at $0.75 \mathrm{~m} / \mathrm{s}$, but there is little difference with the round cross section at the lower velocity.

\subsection{Analysis}

The goal of the analysis is to obtain a reliable approach to classify the cross-sectional shape of the cylinder stimulus from the pressure signature measured by the pressure sensors in a single pass. Preferably, the classification should be insensitive to velocity, size, and separation, indicating that it is truly distinguishing shape. Also, the classification should ideally be simple to apply to new pressure traces. We consider two approaches to classifying the cylinder shape: one based on a few features chosen from specific regions in the pressure traces; and the other based on features extracted with a principal component analysis which are formed from linear combinations of the entire pressure traces. In the first, the features are chosen directly as a result of the averaged results shown in Figure 4-7. In the second, there is no direct influence on which features are utilized.

\subsubsection{Heuristically Selected Features}

Given the observed differences on average between the pressure measured when a square versus circular cross section passes the sensors, the simplest approach to classification is to pick out features among those differences and to assume that they will hold for individual traces. In this way, the maximum before the zero crossing point (Max), the minimum immediately following the zero crossing point ( $\mathrm{Min}$ ), and the slope at the zero crossing point (Slope) were chosen as the characteristic features (Figure 4-8). The slope at the zero crossing point was determined on data filtered with a cutoff frequency of $60 \mathrm{~Hz}$. The length of the wake region was not chosen as a feature, despite the fact that it was identified as a possible source of distinction in the pressure response to the different cross sectional shapes. This was due to its high variability and difficulty in quantifying the feature from individual runs.

The effects of the chosen features are heavily correlated. For example, an increase in the 


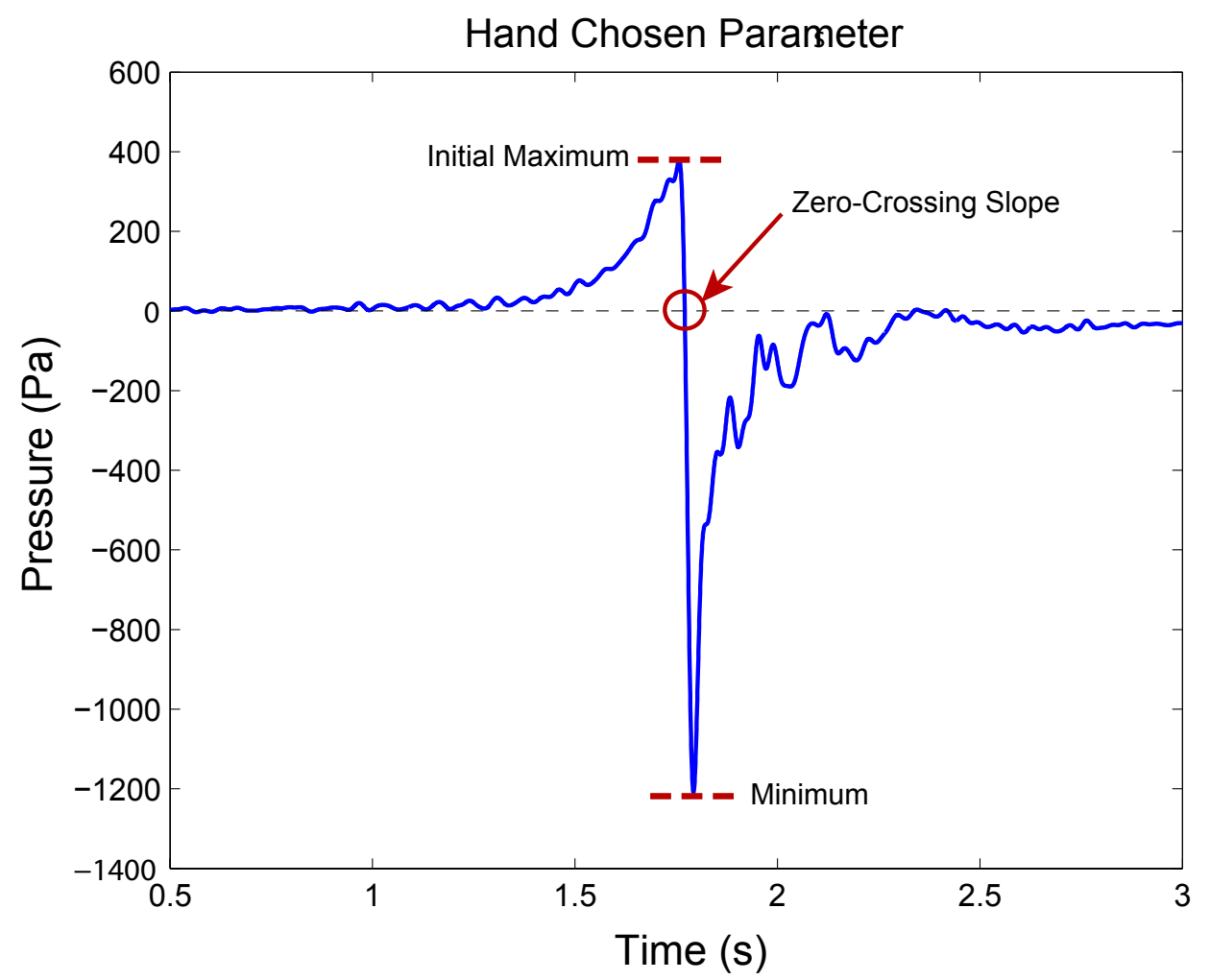

Figure 4-8: Diagram of selected features. The three features initially chosen for analysis are marked on a sample pressure trace. The pressure data has been filtered with a cutoff frequency of $60 \mathrm{~Hz}$. 
velocity of the cylinder would increase the magnitude of all three features but provides no information on the shape. In order to remove some of this correlation, the three identified features were combined into two non-dimensional quantities. The first quantity is a ratio of the minimum after the zero crossing to the maximum before the zero crossing: $\frac{\text { Min }}{\text { Max }}$. For the second quantity, the slope at the zero crossing is made non-dimensional by the peakto-trough change in pressure in unit time: $\frac{\text { Slope }}{M a x-M i n}$. These non-dimensional features have been calculated for every sensor and plotted in Figure 4-9.

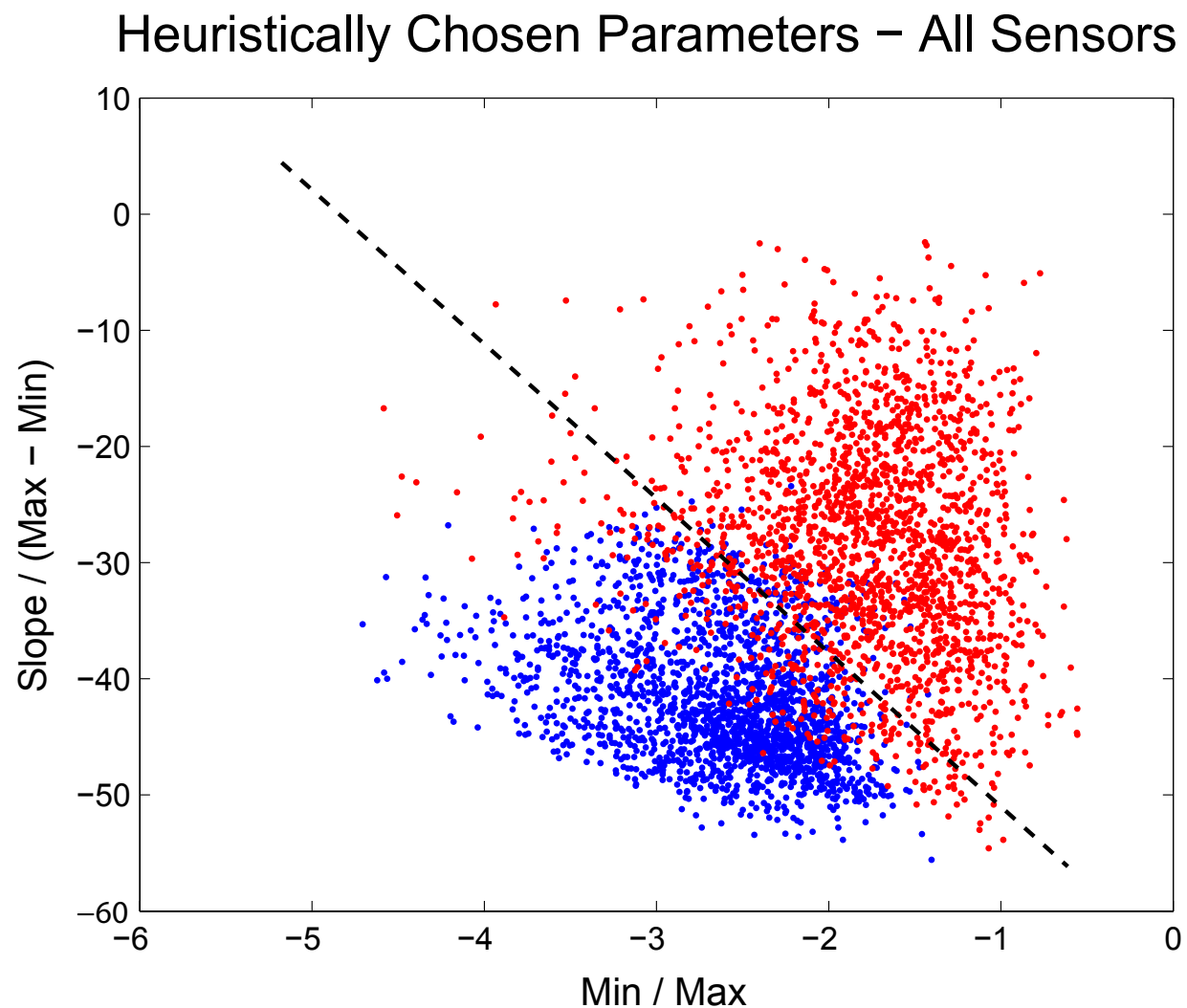

Figure 4-9: Scatterplot of individual traces according to the heuristically chosen features. Blue dots correspond to square cross sections, and red dots to round cross sections. Runs for all velocities, sizes, and separations are included. The decision line based on a sum of squared error criteria applied to a training set from one sensor is marked as a dotted black line. All 4000 datapoints from each sensor in each run are plotted.

The heuristically chosen features divide the points into two adjacent "clouds", corresponding to the two cross sections tested in the experiments, but the two clouds are not completely separable. For simplicity, a linear classifier was used such that all points on 
one side of the decision line are considered to be square and those on the other side to be round (Figure 4-9). The classifier was chosen to minimize the sum of squared errors from a training data subset, where the error for a single run is the difference between a desired distance from the line and the actual normal distance to the line. The magnitude of the desired distance was arbitrarily set to one, since the distinguishing key that the desired distance is positive for one class in the training set and negative for the other class of objects. The decision line is then found from:

$$
\bar{w}=\left(X^{T} X\right)^{-1} X^{T} \bar{y}
$$

in which $\bar{w}$ is a vector of weights that define the decision boundary by $\bar{w} \cdot \bar{x}=0 ; X$ is a matrix made from the features such that each column corresponds to one feature and each row to one test; the vector $\bar{y}$ is the set of desired distances, with elements of \pm 1 corresponding to the class of corresponding test. See [78] for details and a derivation of the result. Minimizing the sum of squared errors makes it possible to obtain a classifier on a set that is not separable without knowing the probability distributions of the features for each class. The percentage of misclassified runs (9.8\%) gives an estimate of the probability of error in the classifier.

These heuristically chosen features are straightforward to identify and do not require any alignment of the data. Given a new time series or spatial distribution of pressure, the zero crossing point is easy to identify, and all other features follow directly from it. This classification approach is also fairly insensitive to different sampling rates, as long as they are high enough to be able to estimate accurately the slope at the zero crossing point. The difficulty with this approach is that there is a fairly large mis-classification rate $(9.8 \%)$, which is likely a result of relying on individual points in a noisy measurement.

\subsubsection{Principal Component Analysis}

An alternative approach to obtaining features is provided by Principal Component Analysis (PCA) (see [78, 40, 38]). This technique, closely related to the Karhunen-Loeve transform and singular value decomposition, obtains optimal features from noisy data. The fea- 
tures are linear combinations of the pressure traces with coefficients that are determined by PCA. The resulting features can in turn be analyzed in a similar manner to the heuristically chosen-features in order to classify the cross sections of the cylinder stimuli.

Principal Component Analysis interprets each pressure trace, obtained from a single run on a single sensor, to be a collection of highly correlated features. Each sample is considered to be a feature. Since there is data from multiple runs, once they are aligned, there are multiple observations of each feature. The purpose of PCA is to compress the data so that a small number of linear combinations of the original features (individual samples) best represent the entire set of data. These new features, which are linear combinations of the sampled points in a pressure trace, are the principal components. They are found so that each principal component has the maximum possible variance, subject to having unit area, and being uncorrelated with all the previous principal components. Implicit in this procedure is the assumption that the information of interest in the data set is that which has the largest variance. For example, it assumes that the difference in pressure traces generated by the different cross sections is larger than the variance in the data due to noise or turbulence. If this is not the case, the analysis would attempt to provide a compact representation of the noise. The constraint of uncorrelated principal components is ideal for feature generation, since it removes redundancy. The variance of the principal components typically drops off steeply, so that the data are well represented by considering only the first few components.

In implementation, PCA works as a singular value decomposition of the sample covariance matrix of the data $([40,38])$. One important detail in the implementation is that the mean of each initial feature (a sample point in the pressure trace) must be removed. This mean is taken across all training data, not over each class. With the means removed, the sample covariance is straightforward to calculate.

Several PCAs were performed on the pressure traces. As mentioned, PCA does not optimize the features for classification. Ideally, the variation in the data would be due exclusively to the difference in cross section shape. In that case, a Principal Component Analysis would produce features well suited for classifying the data by stimulus shape; however, this is not the case. In particular, the variation in the data due to velocity (Fig- 
ure 4-6) is large in comparison to the variation from cross section shape. Therefore it was necessary to normalize each pressure trace by the maximum pressure obtained just before the zero crossing. This effectively removes the scaling influence from velocity, as seen earlier. Also, differences between the individual measurements of the sensors are sufficient to degrade the classification ability of a PCA. Such differences may be caused by variations in the calibration, or in the transfer functions from the ports connecting them to the flow. As a result, the Principal Component Analysis which achieves the best features uses a training set of normalized pressure traces from one sensor. However, although the data from one sensor was used as training data for the PCA, the data from the remaining sensors was used as a test data set on which to examine the effectiveness of the decision scheme.

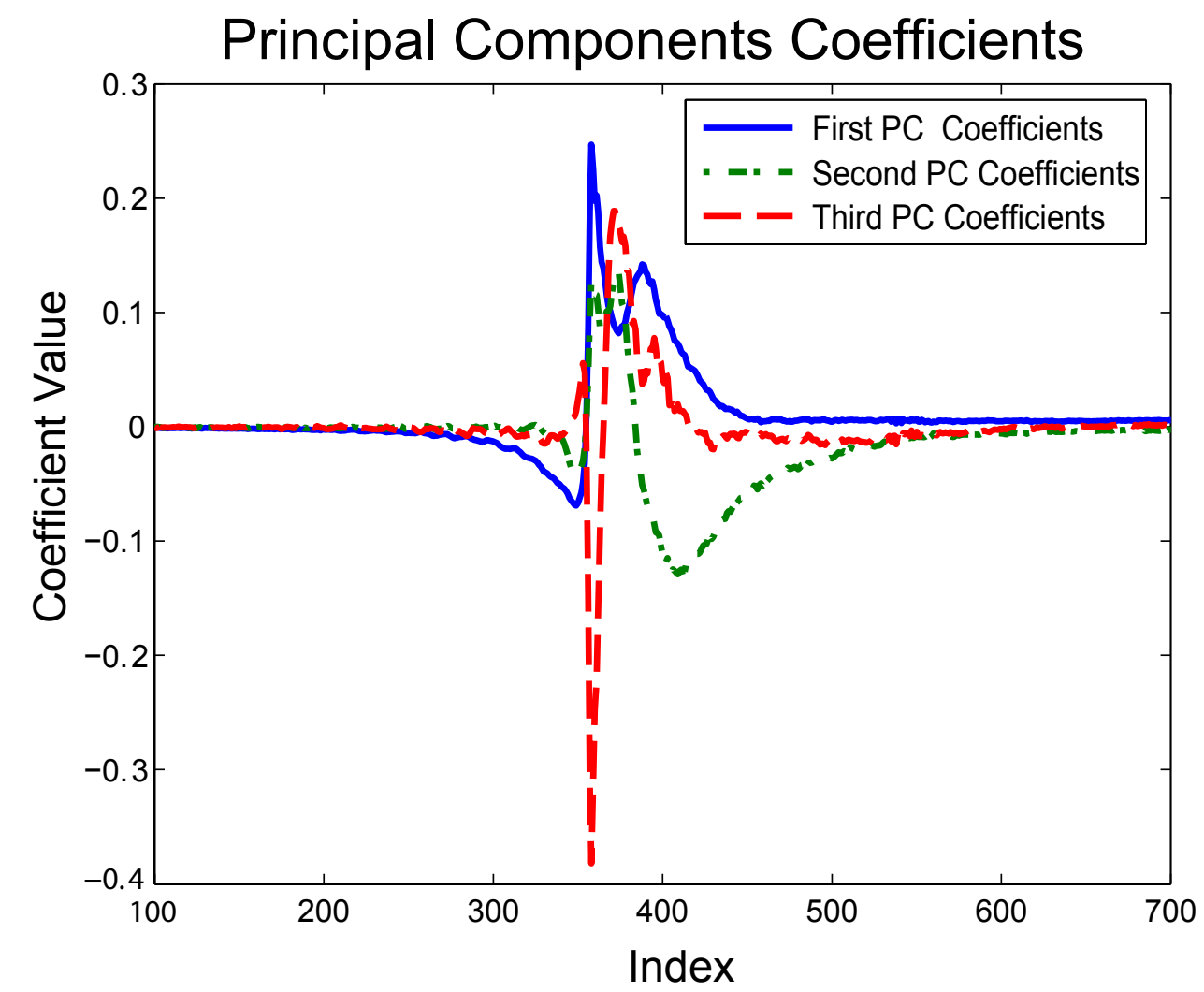

Figure 4-10: Coefficients for the first three principal components derived from the normalized measurements of sensor 2. These are the coefficients for the linear combinations to generate the first three principal components. Data from all experiments were used in the PCA, but only one sensor was used as a training data set.

The coefficients for the first three principal components are plotted in Figure 4-10, or- 
dered by largest variance. Examining these coefficients reveals information about the features identified through PCA. For example, the coefficients for the first principal component are dominated by the region just after the zero crossing. Since the data was normalized by the maximum before the zero crossing point, one could argue that the first principal component is similar to the hand chosen $\frac{\min }{\max }$. However, the principal components are considerably more complex than the heuristically chosen features, since they contain contributions from all parts of the pressure trace, including the wake. This is a known difficulty of the Principal Component Analysis, viz. the difficulty to interpret the physical meaning of each principal component.

Interpreting the coefficients, however, is not necessary for classifying the pressure traces. Using the first three principal components, the data is divided into the two classes of interest. In addition to separating the shapes of the stimuli, the principal components are not strongly dependent on the other variables such as size and velocity. This is evident from the scatterplot of the data (Figure 4-11). The only data clouds formed correspond to the two different shapes; these clouds are roughly ellipsoidal in form. There are also no dense clusters in the clouds that would correspond to differences in the other variables. In the same manner as before, a decision plane minimizing the sum of the squared error can be found based on the training data, and the estimated probability of mis-classification can be calculated (Figure 4-12). Using a Principal Component Analysis to obtain features, the misclassification rate is $1.2 \%$, which is substantially better than the $9.8 \%$ rate obtained with the heuristically chosen features.

\subsubsection{Discussion and Comparison}

Features obtained from principal component analysis do a considerably better job in recognizing the different cross-sectional shapes than the heuristically chosen parameters, although it is slightly more difficult to implement classification with principal components. With the heuristic method, classification involves locating three features in the pressure response. For one of those features a slope must be calculated, but the other two are just the pressure at two corresponding points. These features were drawn from the differences 


\section{PCA of All Data, Normalized}

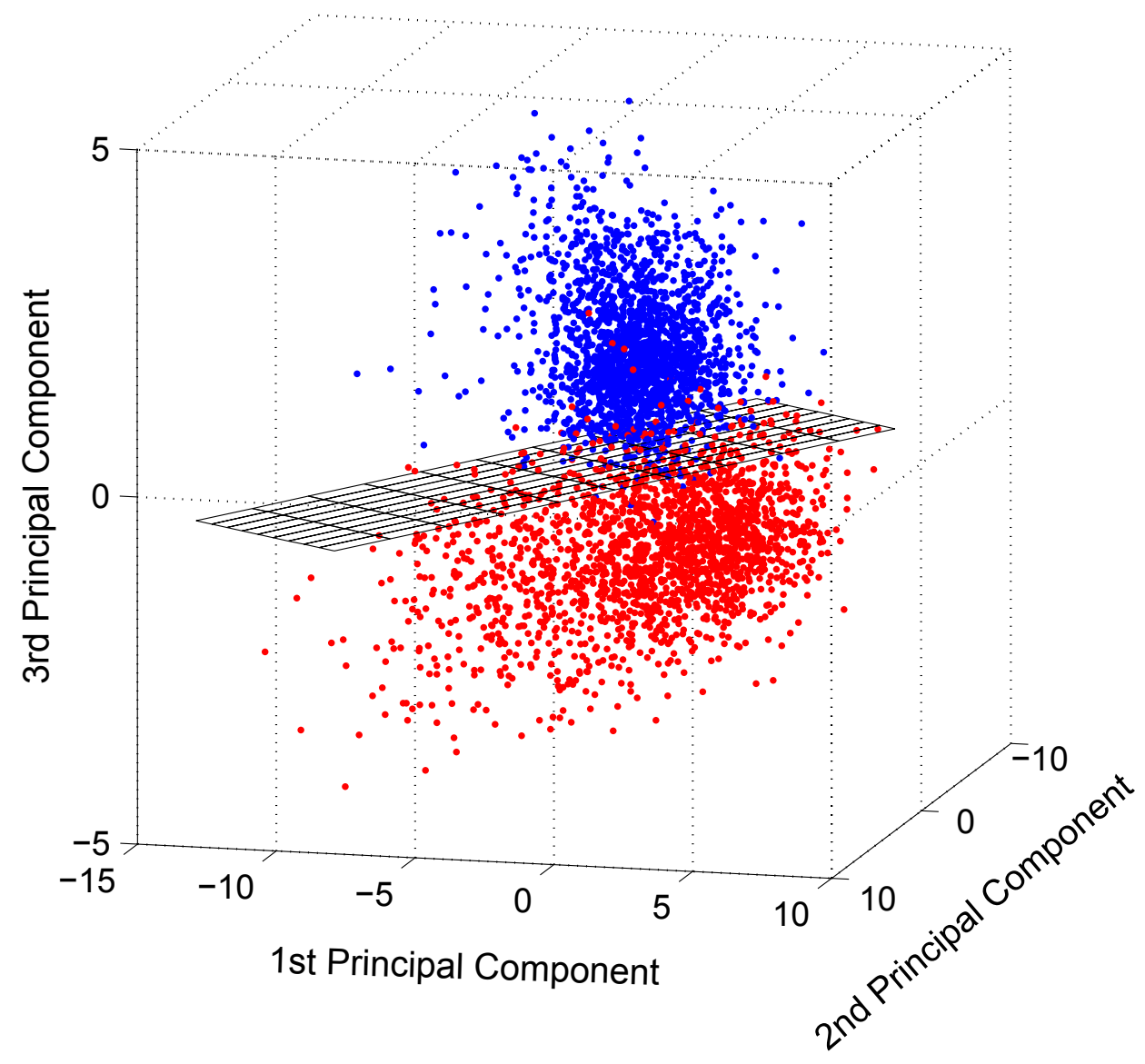

Figure 4-11: Scatterplot of data from all tests and sensors according to principal components. The coefficients for the principal components were found using data from only one sensor. In blue are pressure traces from square cross sections, and in red those from round cross sections. The black plane bisecting the figure is the decision plane minimizing the sum of squared errors. 


\section{Histogram of Normalized Data Using the PCA}

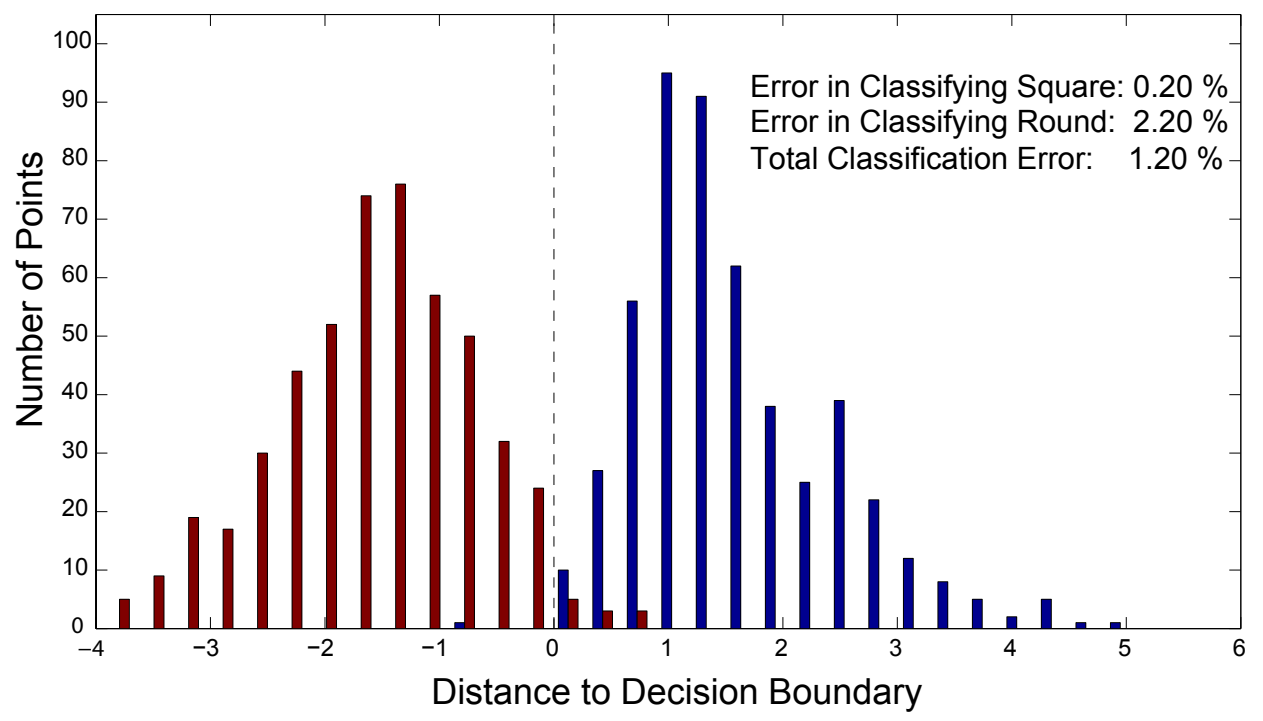

Figure 4-12: Histogram of the distance from the decision plane as shown in Figure 4-11. In blue is the data from the square cross section and in red from the round cross section.

observed in the average pressure traces for each shape. Then, the classification of a new pressure trace can be made based on the sign of the distance $d$, defined by:

$$
d=w_{2} \cdot\left(\frac{M i n}{M a x}\right)_{n e w}+w_{1} \cdot\left(\frac{\text { Slope }}{M a x-M i n}\right)_{n e w}+w_{0}
$$

where the constants $w_{2}, w_{1}$, and $w_{0}$ come from the equation of the decision line in Figure 49, which is $w_{2} \cdot \frac{\text { Min }}{\text { Max }}+w_{1} \cdot \frac{\text { Slope }}{\text { Max-Min }}+w_{0}=0$. The distance will be positive for square cross sections and negative for round cross sections. The main downside to this approach appears to be closely tied to it's ease in calculation. The features used to classify the shape are very localized quantities, therefore susceptible to noise. Low pass filtering is necessary in order to remove some of the worst noise issues and it is telling that this effectively spreads out the number of data points used to calculate the feature.

In the case of the principal components the approach is the complete opposite, relying on differently weighted contributions from the entire pressure data set. There are essentially three steps for classifying a new pressure trace using the PCA results. First the zero crossing point must be located, just as in the other method. Second, the same mean pressure trace 

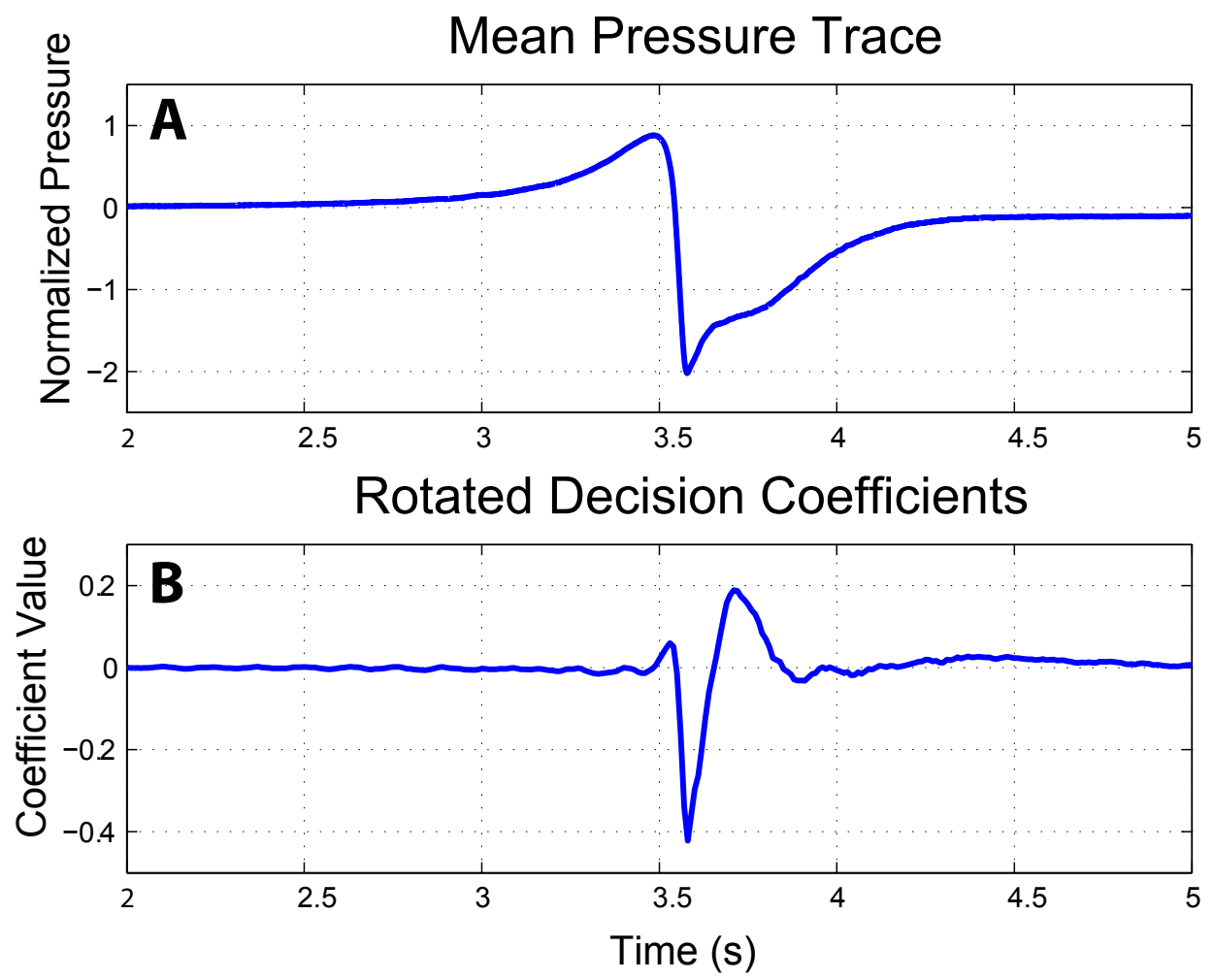

Figure 4-13: A: The mean pressure trace of sensor 2 from all experiments. This mean must be subtracted from any new data before classifying it with the results of the PCA. B: The coefficients for classifying a new pressure trace. These coefficients are the result of rotating the coordinates in Figure 4-11 so that one axis is normal to the decision plane. 
that was subtracted from all the normalized data in the analysis (Figure 4-13A) must be removed from the new trace. Then, the distance to the decision plane can be found through a linear combination with the coefficients shown in Figure 4-13B, which are a combination of the coefficients for the principal components rotated such that they form a coordinate normal to the decision plane. Thus only one linear combination is performed with the new data, and the result is compared to the intersection of the decision plane on that axis. Besides the added complexity, the fact that the mean from the training data must be kept in memory may limit the extent to which this procedure may be generalized.

The primary question remaining on the topic of the PCA results is what information is being used to make the accurate decisions. By rotating the principal components to align an axis with the decision plane, it was possible to condense the decision metric into a single test depicted in Figure 4-13. By examining the weights of this test (part B of the Figure 413) and in particular comparing them against the mean pressure trace (part A), one can extract some intuition on the information being used.

The linear combination weights have three main sections of interest. The first of these is the small positive peak observed just after the 3.5s mark in Figure 4-13B. When compared against the mean pressure trace, this peak is situated between the peak maximum pressure and the zero crossing point. It is expected that the zero crossing point would have zero weight in the classification test, since all the pressure traces were aligned at that point and therefore it is a constant across all the data sets. Similarly, the magnitude of the maximum pressure has been normalized for all the pressure traces, which may be why the test coefficients are small at that location. One explanation for this first peak is that it is tied to the slight rearward shift in the location of that first peak that is observed for the square cross section. It is also possible that the important component is not necessarily the peak of the coefficients, but instead perhaps it is the constant slope in the coefficients through the zero crossing point which approximates the derivative at the zero crossing point. The exact nature of this bump in the decision test weights is still unclear.

The second region of interest in the decision weights is also the most dominant one: the large negative peak in the weights (labeled as III in Figure 4-14). The minimum peak in the weights corresponds to the location of the minimum peak in the average pressure trace. 
This matches the observations in the earlier results section, in which the minimum pressure was the most clear indicator of the shape of the cylinder on average. Since the pressure is negative in this region, the large negative pressure aligned with the weights that occurs with the square cylinder will result in a large positive contribution to the decision metric. The peak in the decision coefficients is sharp in this region, which appears to indicate that the location of the minimum pressure is critical. This is a bit surprising given that the individual traces such as those seen in Figure 4-4 seemed to display a range in positions for the minimum pressure. Despite this, the meaning behind the large weights in this area is fairly clear.

The third region of interest in the decision weights is the most surprising overall. This area is the relatively broad area of positive weights located completely in the wake of the cylinder (see section IV in Figure 4-14). While there are definitely differences that were observed on average in this region of the pressure field, it is also a region of high variability between one trace and the next (see Figure 4-3). As a result, it was unexpected that information from this area would be used to judge the shape of the cylinder. Based on the positive decision weights, this region in the decision weights implies that a smaller or more positive wake region is associated with the square cylinder. It is difficult to see from the averaged pressure traces why this is associated with the square shape. According to the mean traces, the low pressure region for the square cylinder generally takes longer to recover to zero. This would imply the opposite association as is seen is the decision weights, and is also counter to the manner that the decision weights taper off. In any case, the different size cylinders have a much larger effect on the length of the wake than the shape. The only possible feature observed in the averaged pressure traces that might be associated with this region in the decision weights is the slight rebound in the pressure seen after the primary negative peak for the square cylinders. In Figure 4-7, there is a consistent region just after the main negative peak in which the pressure for the square cylinder is larger than that of the round cylinder. It is a brief period and not a large effect when viewed on average, but that may be what the weights from the PCA approach are using.

These three regions describe the main sections of non-zero weights in the classification test. The regions before and after these three sections appear to be largely zero. However, 


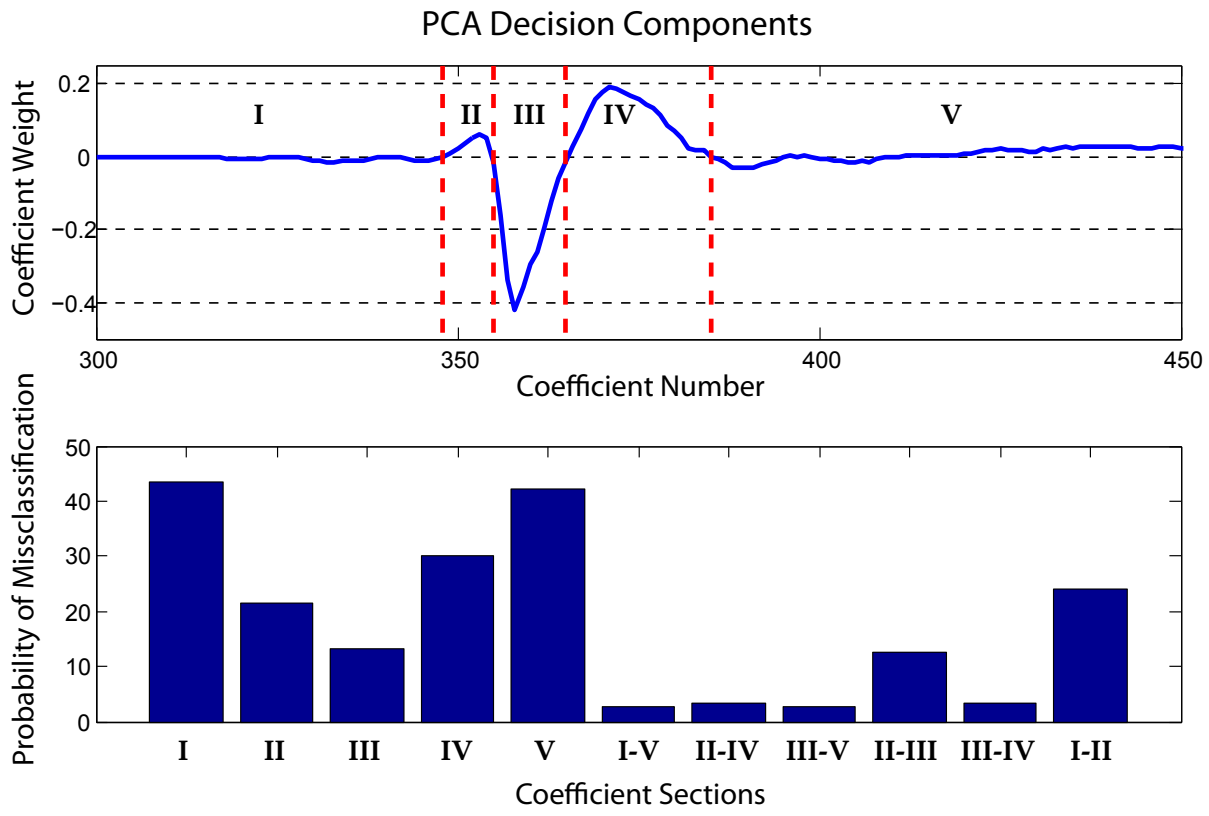

Figure 4-14: Comparison of results using modified decision weights. The top subfigure indicates the division of the decision weight coefficients into different regions. A classification test using weights composed of the original coefficients with regions zeroed out is shown in the lower subfigure. The non-zero regions are labeled for each bar.

since they span a large area, they may still contribute to the final decision. In addition, although each of the three primary sections can be tied to possible differences between the shape pressure fields, it is important to recall that the PCA methods used to create the decision weights did not necessarily prioritize the shape. In particular, it is possible that the third region described, found in the volatile wake are of the pressure field, does not affect the shape classification but was instead included in the principal components simply due to the high variability. In order to examine the importance of the various sections of the weights in the classification test, the misclassification rate was recalculated based on tests different sections of the decision weights set to zero. The results are visible in Figure 4-14. In the lower figure, the first five bars indicate the results when each section individually is non-zero. Compared to the full result (the sixth bar at $1.2 \%$ error), all the individual sections are substantially worse at classifying the shapes. Keep in mind that a fully random decision metric would give approximately 50\% error. Therefore sections I and V individually are fairly close to providing no information about the shape of the cylinder. This confirms the 
expectation that the small variations in the weights in these sections do not substantially effect the classification. When considered individually, the best classification region is region III, which again matches expectations. It is interesting to note further, however, that region II more accurately predicts the shape than region IV. Upon further investigation, the simple relationships implied by the performance of the individual sections is substantially complicated. First of all, it appears that the information utilized in section II is redundant with that of section III, where as the information in section IV is crucial to an accurate classification. These statements come from the observation that classification based on sections II and III jointly result in fairly poor accuracy, where as the inclusion of section IV improves the result to slightly worse than that of the full set of coefficients. On the other hand, when including section IV, there is very little difference in the results when section II is included or excluded. It is also interesting to note that the inclusion of the final section of coefficients in the III-V result provides the best result by a small margin. In comparing the results of the III-V section to the I-II section, it is clear that the coefficients beyond the zero crossing point are much more important than those before. In summary, it appears that sections III and IV are critical in producing an accurate classification, where as section II is redundant with III, and section V still provides a very modest benefit.

Having established that the pressure distribution in the wake is a vital component in the decision to classify the shape of the passing object, it is still unclear how the information about the shape is encoded in the wake. One possibility that deserves exploration is that the structure of the shed vortices changes between one shape and the next. It is well known that the shape of the cylinder affects the Strouhal number [5], which governs the vortex shedding frequency. It is possible that the spacing in the shed vortices consistently effects the measurements, even if the phase of the vortex shedding is random. For the range of Reynolds numbers used in the experiments, the Strouhal numbers for both cylinder shapes are fairly flat at 0.2 and approximately 0.14 for the round and square cross sections respectively. This means that the vortices are spaced at approximately every 2.5 diameters for the round cylinder and 3.6 diameters for the square cylinder. When considering the different diameters included in the test data, the results are shown in the following table:

Note for reference that the length of the sensor array is about $20 \mathrm{~cm}$. Therefore, in most 


\begin{tabular}{|l|c|c|}
\hline & $7.62 \mathrm{~cm}$ Diameter & $5.08 \mathrm{~cm}$ Diameter \\
\hline Round & $19 \mathrm{~cm}$ & $12.8 \mathrm{~cm}$ \\
\hline Square & $27.1 \mathrm{~cm}$ & $18.2 \mathrm{~cm}$ \\
\hline
\end{tabular}

Table 4.2: Table of the Strouhal numbers associated with the cylinder identification experiments.

of the experimental runs a vortex was shed near at least one of the sensors. However, the more important observation from the table is that the effect of the two different diameters on the Strouhal number is large compared to the effect of the shape on it. Although for a given diameter, the vortex spacing is much larger for the square cylinder, the vortex spacing of the small square cylinder is nearly equivalent to the at of the large round cylinder. This means that half of the data would be ambiguous or even potentially misclassified if the test was based solely on the vortex spacing. When in addition to this, the random phase of the vortex shedding is factored in, is unlikely that the vortex spacing is a contributing factor in the cylinder shape identification. The cause for the difference in Strouhal numbers with the cylinder shape is related to the distance between the two separated shear layers[5]. This width is in turn increased for bluffer bodies such as the flat face of the square used in these experiments. It is possible that this difference in wake size and structure is responsible for the information utilized in section IV of Figure 4-14. The wake thickness would have the same dependence on shape and size as the Strouhal number, but would not rely on the added random timing of the vortices. When considered alone, the decision coefficients in section IV resulted in approximately $30 \%$ error in classification. This matches the hypothesis that the wake thickness would distinguish approximately half of the data and be ambiguous on the other half.

\subsection{Conclusion}

This chapter has shown that the shape of a cylinder can be distinguished between two known shapes using the information from a linear array of pressure sensors. Although this does not go the entire way to demonstrating that an arbitrary shape can be identified based on a lateral-line-like sensor array, it has established that detailed information on 
the shape is available and indicated what aspects of the pressure response are the most relevant. Two successful approaches for distinguishing the cylinder shape were described in this chapter, overcoming the large variation observed in repeated trials with the same cylinder. The first utilized differences observed in the averaged responses to each shape in order to select features that could identify the shape in an individual pressure trace. The second approach, with an error rate less than $2 \%$ utilized a principal component analysis to identify the modes of most significant variation in a set of training data. By ensuring that the difference in shape was the primary cause of consistent differences in the data, this approach was directed towards generating a single test which evaluates the shape of the cylinder. The result from the PCA approach utilizes the entire pressure data set of the cylinder after is has passed to make a decision.

The accuracy in the classification based on the PCA-derived test is all the more surprising given the range of velocities, sizes, and distances of the cylinders used. Although the primary interest is in the shape of the cylinders, it is important to include this range of parameters in any attempt to demonstrate that it is the shape that is being distinguished instead of two experiments. For example, the importance of the Strouhal number could not be evaluated without the variety of sizes in the cylinders tested. The two tests described in this chapter were both able to identify the shape of a cylinder after it had passed the sensor array using a single test to determine the shape in a range of conditions. While this should not be generalized into concluding that the tests work for all sizes and velocities, it is important to know that a test will be robust to small errors in the estimate of the size or velocity that might be predicted ahead of time via strategies from Chapter 3.

The results from the classification test developed with the principal component analysis makes it clear that the pressure corresponding to the wake region in the pressure field contains significant information about the shape of the cylinder. In fact, it appears that the PCA results were equally effective in identifying the shape if no information forward of the zero-crossing point was used. This is an interesting result in that it runs counter to the implicit assumptions of potential flow studies that portions of the flow, such as that ahead of the cylinder, may be well represented by the potential flow about an object. Given the importance of the wake in identifying the shape between two known possibilities, it will be 
necessary in order to identify an arbitrary shape to have a good flexible model for the wake and its relationship to the shape. This may call for numerical estimation methods. From looking into possible sources of information in the wake, the results have reinforced the importance of first estimating the size of the cylinder in order to be able to distinguish the cause for a large wake width. 


\section{Chapter 5}

\section{Vortex Tracking}

\subsection{Introduction}

From the results in Chapter 3, it was found that the immediate wake of a moving cylinder contained a substantial amount of information for distinguishing the shape of that cylinder. In this chapter, the wake is considered on its own, with an emphasis on a well developed wake further from the body that have generated it than in the moving cylinder case. Within the wake, vortices are a critical long-lived component that forms due to instability in the separated shear layers [5]. When considering the strict range limitations due to the decay of the near field pressure distribution, vortices present an opportunity to extend the footprint of a moving object much further. For example, a seal has been shown to be able to trace the motion of remote underwater vehicle based on the wake with a delay of up to five seconds [29]. Information gathered about vortices in the wake of an object can be used to infer certain features of the original object, such as speed and size based on the vortex spacing and strength.

Completely separate from their relevance to detecting and identifying a moving object, vortices are of interest in themselves. As persistent coherent fluid structures, vortices affect the flow and pressure distributions about nearby objects. Besides being the basis for the entire idea of using an artificial lateral line, this also means that vortices affect the function of flapping foils or control surfaces which rely on generating a pressure difference between opposing surfaces. Therefore, self generated vortex can have substantial effects on maneu- 
vering vehicles. As one example, the leading edge vortex that forms in a foil at high angles of attack strongly influences the amount of lift generated, critically important for the flight of insects such as the Hawkmoth [1].

In addition to the background described in Chapter 2 on how a fish is theorized to locate and utilize vortices in the wake of an object to decrease its energy expenditure, there have been a few studies examining the problem of tracking vortices based on distributed pressure sensors or artificial lateral lines. In the first of these, Ren and Mohseni [66] examined the detection of a vortex wake from a fish-like body in two dimensions. Their study, using potential flow in two dimensions with a Joukowski foil model for the fish body, was largely focused on the conditions for identifying a regular convecting vortex street based on the pressure and pressure differences on a sensor array distributed over the boundary of the foil. However, the spacing between the vortices considered in this work is much smaller than the array length, so that five or more vortices are contributing substantially to the measurements at any particular time. The focus is correspondingly on the effect of array density in capturing the oscillatory pressure gradients of the problem. This work differs from that presented in this chapter since this chapter is concerned with identifying the precise state of one and two vortices, as opposed to the global characterization (such as frequency) of a vortex wake, which can be subsequently extracted from the detailed information.

Suzuki and Colonius [74], consider the inverse problem of identifying a vortex in an air channel from a numerical perspective. The estimation was accomplished via an instantaneous least squares optimization, with a small discussion of a fixed estimator-corrector mechanism between timesteps. The enclosed channel with known flow velocity considered in the paper is a substantially different environment, particularly in terms of the convention of the vortices. The paper also tackles the question of tracking multiple vortices simultaneously, finding that the accuracy of the vortex tracking based on the least squares inversion degrades substantially when two vortices are in sufficiently close proximity to affect the each other's measurements.

Both Suzuki and Colonius, and Ren and Mohseni demonstrate that in a potential flow formulation, information on vortices in the flow is theoretically available to a lateral-linelike sensor array. However, it is still unclear to what extent these theoretical results extend 
to real environments. This is particularly true given the ignored viscous affects and other background fluid flow which may lead to highly correlated noise in the measurements. This chapter develops a slightly different theoretical base from both previous works, using the same potential flow assumptions, in order to solve the inverse problem for an artificial lateral line tracking a vortex in an open environment from both a fish (or AUV) and ship hull perspective. This model base and estimation method is tested on experimental data using single vortices and vortex pairs, using particle image velocimetry (PIV) to independently verify the accuracy of the inversion. The use of experimental data also makes it possible to verify the accuracy of the forward model, which predicts the pressure distribution based on a known vortex state. In addition, the questions of tracking two interacting vortices and the relation between sensor array parameters and accuracy are considered.

\subsubsection{Variable Definitions}

For consistency throughout multiple experiments in tracking vortices, similar variables and coordinate systems will be used in each case. The general problem formulation consists of one or several vortices moving in a plane, with some points of measurement in a linear array on that plane. The axes are defined with the origin at one of the sensors in the linear array, with the $X$ axis parallel to the linear array and the $Y$ axis pointing away. The fluid domain in which vortices may be is limited to the half plane defined by the positive $Y$ axis. Each vortex is defined by a state vector of three variables: $\left(x_{i}, y_{i}, k_{i}\right)$ where $x_{i}$ and $y_{i}$ are the position of the $i$ th vortex, and $k_{i}$ refers to its strength which is related to the circulation of the vortex by $\Gamma_{i}=2 \pi k_{i}$. Frequently the position of a vortex is depicted as the complex $z_{i}=x_{i}+\imath y_{i}$. The position of each sensor is denoted by $s_{j}$, where $s$ marks a location on the $x$ axis. The pressure measured at one of the sensors is $P_{j}$, with the subscript corresponding to the sensor number, as for $s_{j}$.

When considering models for the vortices, the vortex state vector will be referred to as $q_{i}(t)$, where the subscript refers which state formulation is being used. All cost functions are denoted by $J$, and are positive functions of vortex parameters. For a general variable, a superscript of "meas" refers to the measured quantity, as opposed to the modeled quantity 
(denoted by a "model" superscript) or a true quantity (superscript "true") in the case it is being compared directly against a simulation with a defined true value.

\subsubsection{Vortex Modeling}

The vortex tracking described in this chapter is founded on a general set of models using potential flow in two dimensions to represent the real fluid domain. The two major assumptions involved in these representations, that of an inviscid and largely two dimensional flow, fit well for this problem as long as a few restrictions are placed on the vortices.

Unlike the situations described for cylinder tracking and identification, the fluid flow involved with measuring vortices is very well captured by potential flow models. The inviscid assumption is substantially less onerous. Due to the simple streamlined geometries of the sensing bodies, there is no flow separation on the bodies based on the flow induced by vortices at a reasonable distance. For the vortices themselves, since they are purely fluid structures there is no wake or flow separation as there is with a moving cylinder. Therefore, in a two dimensional plane, a line vortex described by the complex potential $\Phi(z)=$ $-\imath k \log \left(z-z_{o}\right)$ accurately predicts the flow distribution beyond a minimum distance from center of the vortex. A core region of the vortex must be exempted as the line vortex model predicts that the velocity is unbounded. Instead, viscous effects dominate close to the vortex center, and the rotation is close to a solid body rotation. Several models are commonly used to account for the vortex core, such as the Rankine vortex model which inserts a solid body rotation within some boundary radius, and the Oseen vortex [68], which further smooths the transition between the solid body and irrotational flow.

In large part however, the vortex core can be ignored for the purposes of tracking vortices. The vortices considered here are generally well separated, with the distance between vortex centers substantially larger than a vortex core diameter. In addition, as long as the vortices do not approach too closely to the sensing body, the vortex cores do not affect the pressure measurements. This is the case generally for all the experiments in this chapter.

The only remaining concern in terms of viscous effects is the generation of secondary vorticity from the interaction of a vortex with the sensing body. This will occur if the pri- 
mary vortex approaches the sensing body structure too closely and causes flow separation. Based on observations, this did not occur except at distances in which the influence of the core on the measurements might also be suspected (based on PIV results). Therefore, the experiments and analysis of vortex tracking were limited to trials in which the vortices maintained sufficient distance.

The two dimensional limitation of the models used in this chapter is a potential source of error when estimating the vortex state. In order to minimize the discrepancies caused by this assumption, long vertical vortices were generated using extended foils. While technically vortex rings, this approach maximizes the distance between the horizontal portions of the ring and the sensors. In addition to the vortices themselves, the shape of the sensing body affects the amount of flow out of the modeled plane. This affect will be considered later, as it affects the experiments differently.

\subsection{Experimental Procedure}

In all of the experiments involving tracking a vortex or vortex pair based on a linear pressure sensor array, the basic layout is the same. The experiments consist of two parts: measuring the pressure generated by vortices with a lateral-line-like sensor embedded in an enclosure, and completely independently collecting information to locate and measure the strength of the vortices. These two components allow the estimation of the vortex state based on the pressure measurements to be compared against a ground truth. The independent determination of the vortex state is done using particle image velocimetry (PIV), which provides a quantitative description of fluid flow in a plane. The primary difference between the two sets of experiments is in the shape of the body which encloses the sensors. One set of experiments uses a streamlined cylindrical body, much like the shape of the Remus AUV [37] and the other uses a flat plate in order to approximate the effect of sensors mounted on a ship hull. 


\subsubsection{Particle Image Velocimetry}

Particle image velocimetry (PIV) is a common fluid visualization technique that has developed many variants since its introduction. In general it provides a quantitative estimate of the velocity field of a fluid. In these experiments, PIV is used in both experimental setups to provide the control information for the vortices being tracked. Although the details of the implementation differ slightly for each setup, the fundamental technique of PIV is the same. In each case, a very thin laser sheet is used to illuminate a horizontal twodimensional plane in the flow, in line with the sensor array axis. Inside this plane, neutrally buoyant small particles reflect the light while effectively traveling with the fluid. A camera located perpendicular to the laser sheet captures images of the position of these particles in rapid succession. The change in location of the particles between frames then forms the basis for the calculation of the velocity field. Instead of tracking individual particles, the velocity field is determined by comparing the cross correlation of small windows in each frame. This makes use of multiple particles to estimate the velocity direction, and is more robust against particles traveling out of the imaged plane. The size of these interrogation windows is an important factor in the processing of the PIV data.

The planar particle image velocimetry used in these experiments provides an estimate of the velocity field in the illuminated plane. From the velocity field, it is possible to identify the location vortices (discussed further in 5.3.2). One important constraint is that no information is available on velocity components out of the illuminated plane. In order to minimize the importance of this velocity component, the experiments, particularly with respect to the vortex generation, were designed in order to maximize the two-dimensional nature of the flow.

\subsubsection{Experimental Layout: Streamlined Body}

The primary characteristic of the first experiment for tracking vortices is that it utilizes a streamlined body to enclose the pressure sensor array. The basic geometry of the sensing body consists of a cylindrical body with a $10.16 \mathrm{~cm}$ ( 4 inch) outer diameter. The front and back of the tube are capped using sealed plywood attached to high density polyethylene 


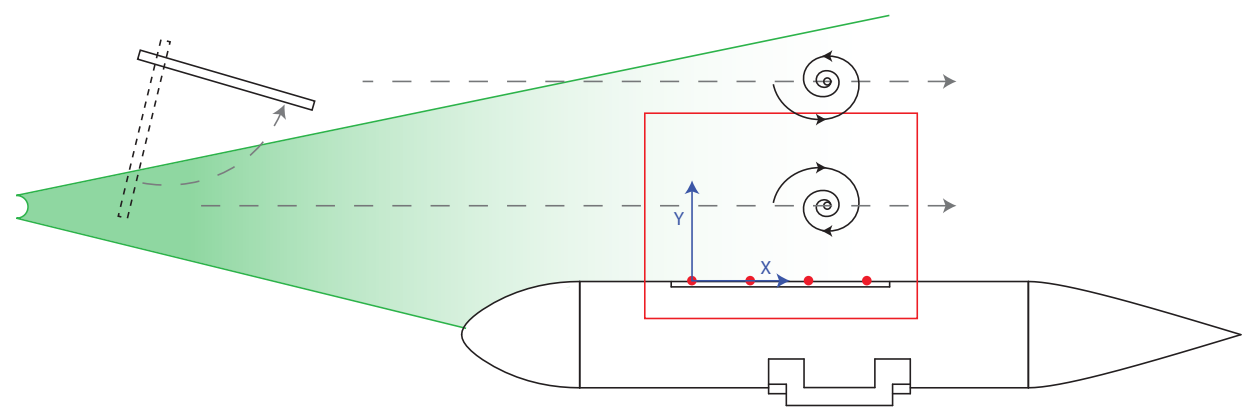

Figure 5-1: Depiction of the experimental layout for tracking a vortex pair near a streamlined body. The red box marks the imaged region for PIV, which is lit by the laser in green. The location of the sensors are marked by red dots.

inserts. The nose of the body is shaped into a roughly parabolic cone smoothed into the cylinder, and the tail of the body is a straight cone with a rounded end. The body used in these experiments is the same as that described for Chapter 4, but the sensors used are slightly different.

This structure, visible in Figure 5-1, contained four Honeywell 19C015PG4K pressure sensors spaced $6.35 \mathrm{~cm}$ (2.5 in) apart in a line. These sensors were ported to the external flow in a manner designed to reduce noise. The sensors themselves were connected to a small rigid tube with $0.472 \mathrm{~cm}(0.186 \mathrm{in})$ inner diameter, but the final port through the housing consisted of a smaller hole $1.3 \mathrm{~mm}(.050 \mathrm{in})$ in diameter and $3.2 \mathrm{~mm}(0.13$ in) deep. The small size of the final opening is necessary in order to reduce the amount of averaging and interaction between the external flow and the port. Additionally, it was found to be critically important that all the connections between the sensors and the flow be rigid and short, in order to reduce the noise or signal modification caused by the connections. In order to maximize the sensitivity of the pressure measurements, the cavity between the pressure sensor and the port to external flow was manually flushed and filled with water (using a syringe) before experiments. It was found that leaving this cavity full of air substantially reduced the amplitude of the pressure signals. Besides the simple sensitivity observation, there are concerns that the air-water interface at the port would affect the pressure measurements, since the changing diameter of the pathway in conjunction with the moving interface would lead to pressure imbalance across the interface. The signals 
from the pressure sensors were amplified immediately within the sensing body, using the amplification circuit described in Chapter 3. The resulting amplified signals were transmitted through a short shielded cable to a NI-DAQ USB 6210 data acquisition system powered directly by an unplugged laptop. The data was collected at $30 \mathrm{kHz}$ via a custom LabView program. Besides the pressure data, an additional signal from the PIV system corresponding to the camera trigger was also recorded, in order to later match the timing between the two data sets. To calibrate the sensors, the change in static pressure between the operating depth and the surface was used, based on both the initial setup and the final removal.

In order to generate vortices easily detectable by the pressure sensor array, a vortex pair was generated by the sweep of a thin rigid flat plate (roughly $23 \mathrm{~cm}$ (9 in) by $28 \mathrm{~cm}$ (11 in) with the axis of rotation 2 inches from the rear). Figure 5-1 depicts the rough orientation of the plate at the beginning and end of the motion used to generate the vortices. In order to provide the torque to generate large vortices, it was found that driving the plate by hand was much more effective than all attempts to mechanize the process. This required some training in order to properly direct the vortex pair, which was accomplished using the illumination PIV laser sheet at very low power to provide feedback and visualization of the fluid motions. The end result, as shown in Figure 5-1, is that a pair of vortices was regularly generated which would travel in straight line roughly parallel to the direction of the sensor array.

These experiments were carried out in a small fluid visualization tank at the MIT towing tank with dimensions of $2.4 \mathrm{~m}$ by $0.75 \mathrm{~m}$ by $0.7 \mathrm{~m}$, with the last dimension of height varying slightly depending on the water level. The depth of the line of pressure sensors below the water level was approximately $30 \mathrm{~cm}$ in all cases. The calibration of the sensors was checked each day of experimentation by comparing the pressure difference from the surface to the final operating depth. The sensor body was mounted in the tank using a stand which extended slightly behind the streamlined body. In order to minimize any motions, the stand connected to the tank in three non-collinear locations forming a rigid connection.

The PIV setup used to independently image the vortex state utilized a Quantronix Darwin 527 Series Nd:YLF laser mounted on an optical table at one end of the tank. This is a pulsed laser with an optimal frequency of $1 \mathrm{kHz}$ for maximizing the output power. In these 
experiments, a frequency of $300 \mathrm{~Hz}$ was used. The laser was converted into a spreading light sheet using a simple D-cylinder lens. In the experimental configuration, the laser sheet was obscured by the plate used to generate vortices at the beginning (Figure 5-1). However, at the end of the motion the plate is completely out of the path of the laser, and the delay in fluid motion due to the separation between the plate and the sensing body meant that this was not a practical concern. The flow was seeded with ceramic spherical particles AGSL150-30TRD by Potters Industries Inc. These particles have a mean size of 100 microns and are coated with silver ( $30 \%$ by weight) while being close to neutrally buoyant. The silver coating of the particles increased the reflectivity, overcoming the lower power of the laser at the low frequency. The high speed camera used to image the particles (La Vision Imager Pro HS) was mounted below the tank, looking up through a window in the base using a $50 \mathrm{~mm}$ lens. This approach avoided the concern of imaging through the free surface.

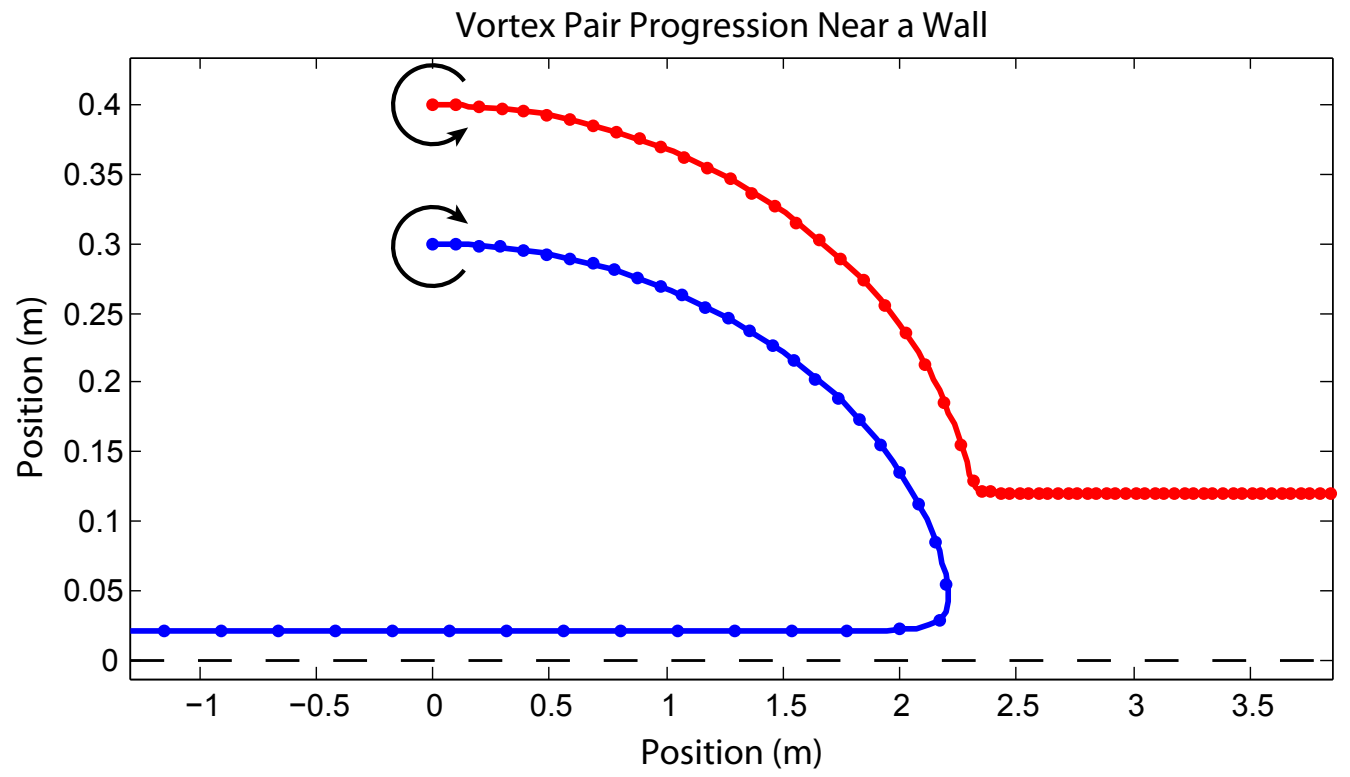

Figure 5-2: Simulation of the path of counter-rotating vortices of equal magnitude near a wall. Dots are placed at equal time intervals along the path to depict the speed. The wall is marked by a dotted line at the $y=0$ axis. 


\subsubsection{Experimental Layout: Flat Body}

In the case of the sensor array being embedded in a flat plate, which more closely reflects the situation on a ship hull than the streamlined sensor body, the same experiment cannot physically be carried out. With a flat plate sensor enclosure, the interaction between the vortices and the plate alters the path of the vortices. Although the setup of the second set of experiments (Figure 5-3) uses a flat plate of limited size, it effects the vortices in much the same way as a flat plate of infinite span. Using the potential flow line vortex model, the flat plate can be accounted for by reflecting the two real vortices over the plane of the plate. This four-vortex system now interacts very differently than a two-vortex system. In particular, if the two real vortices begin with equal and opposite circulation, the velocity of the two vortices will not be equal: The vortex closest to the wall will be affected by the image vortices more strongly. Since the closest image vortex is it's own reflection, the dominant influence is to slow down with respect to the outer physical vortex. This implies that the case of linear translation for the vortex pair parallel to the wall in an unstable scenario. It can be accomplished in potential flow by just the right balance of circulation between the vortices, but it would be very unlikely to occur in experiments.

Figure 5-2 demonstrates the type of path that results from a vortex pair with equal circulation magnitude and initially located perpendicular to the wall. As seen in the figure, the two vortices first rotate and converge on the wall. At a certain point in the approach to the wall, the distance of the closest vortex to its image becomes smaller than the distance to the second physical vortex, resulting in a change in direction. Fairly quickly after this, the two physical vortices proceed as if nearly independent of each other, following paths parallel to the wall in opposite directions, at speeds corresponding to their distance from the wall. Experimentally, based on PIV observations, the vortices were verified to carry out this prediction. In order to generated data to test the tracking of vortices near a hull-like body, an alternative configuration was used, where the initial vortex pair is arranged parallel to the wall. In this case, the two vortices approach the wall directly and split evenly, both final vortex trajectories having the same distance from the wall, but moving in opposite directions. The rough observed trajectories are sketched in the layout diagram (Figure 5- 
3). Due to the limited span of the sensor body, the initial vortex trajectory is positioned close to the edge of the wall, so that one vortex is effectively peeled off and sent in front of the sensors. As might be expected from this arrangement, the pressure measured by the rightmost few sensors is initially contaminated by the second vortex.

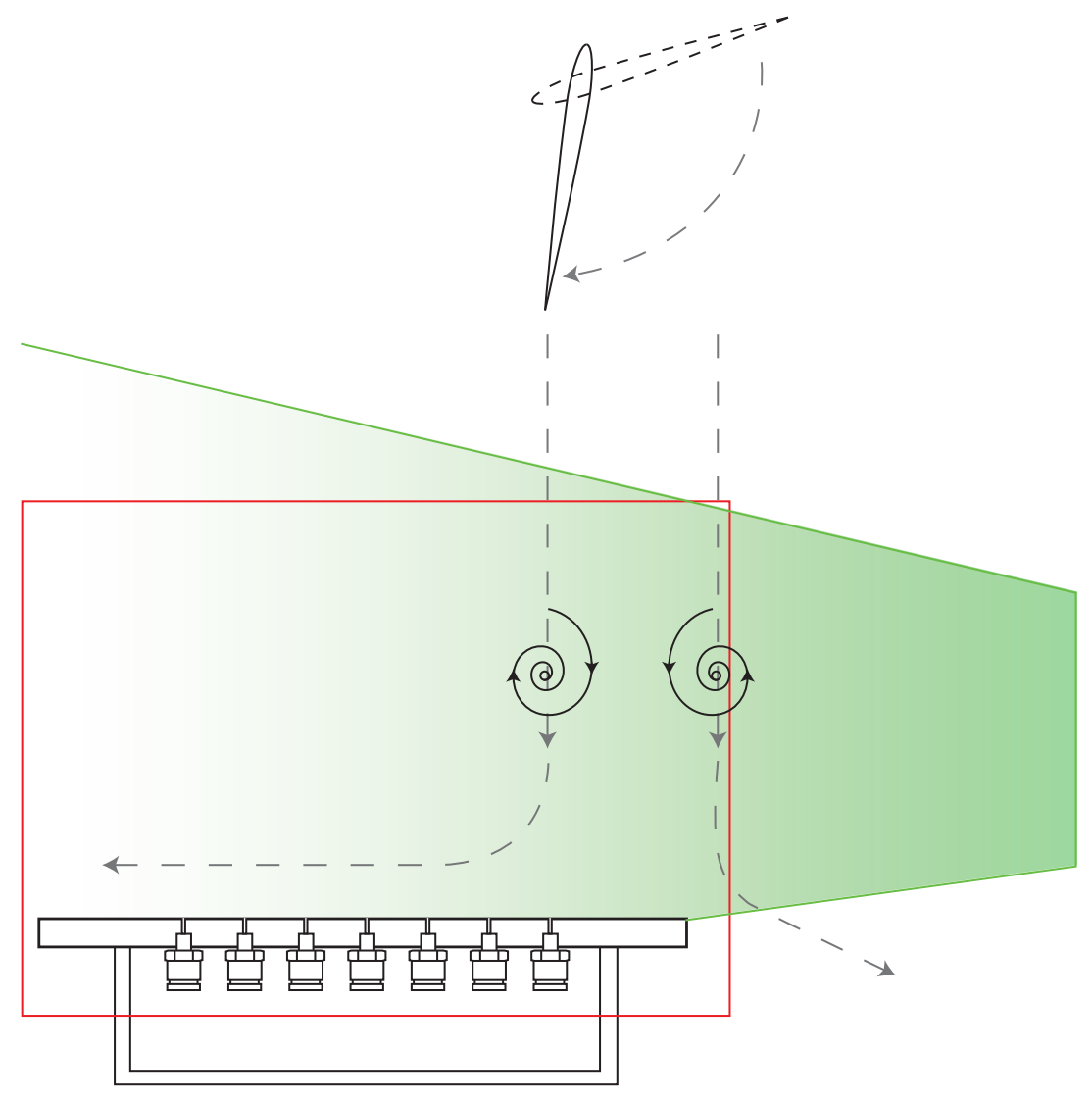

Figure 5-3: Depiction of the experimental layout for tracking a vortex pair near a flat plate. The red box marks the imaged region for PIV, which is lit by the laser in green. The location of the sensors are marked by red dots. A rough approximation to the path of the vortices is also shown.

For the experiments on a lateral-line-like sensor embedded on a flat surface, the vortices were generated by the rotation of an 8 inch chord, 36 inch span foil made of aluminum, with the axis of rotation 2 inches from the front leading edge. The foil cross section was a standard NACA0008, with a rough maximum thickness of 0.6 inches. The large span of the foil served to create a pair of vortices that can be more accurately modeled in two dimensions. This foil was mounted vertically with the sensors aligned near the mid-span 
level. A rod connected to the foil forming the axis of rotation was held in the motor mount above the water in order to allow for persistence in the location of the foil. However, the actual motion of the foil was hand driven, using a small bar attached obliquely to the foil to apply torque. This apparatus was necessary to provide sufficient torque to generate clearly visible and measurable vortices. Unfortunately, as a result of the hand driven motion, an exact description of the motion of the foil is unavailable and subject to a certain degree of variability between trials. The results, as observed through PIV images, corresponded with well defined measurable vortices most when the foil initially rotated counter the final motion. Roughly speaking, the foil would begin from rest at approximately 80 degrees from the axis pointing perpendicularly to the linear array, slowly sweep back to approximately 100 degrees, then smoothly but quickly rotate to its final position of 10 degrees.

The sensor body and data acquisition setup used in these experiments is identical to the one described in Chapter 3, in the experiments in cylinder tracking. In short, seven Honeywell 19C015PG4K sensors formed a linear sensor array, spaced apart by 0.75 inches. The linear array was embedded in the center of a 16 inch square flat plate, representing a ship hull or section of another large flat object. The same amplification circuit was used to amplify the pressure signals 970 times before collecting the data via a NI-DAQ USB6210 analog to digital converter. Pressure data was collected at $30 \mathrm{kHz}$.

The flat surface vortex estimation experiments were done in a different tank compared to the streamlined body experiments. This tank is the same as that discussed for the experiments in Chapter 3, with dimensions $3.6 \mathrm{~m}$ by $1.2 \mathrm{~m}$ by $1.2 \mathrm{~m}$. The calibration of the sensors was again done by comparing the difference between the pressure at the operating depth and the surface to the operating depth (approximately $40 \mathrm{~cm}$ ). This calibration was checked at the start of the experiment and again at the end, to check for any long term drift or other malfunction.

The PIV setup for the flat body experiments is also substantially different than that of the streamlined body, due to the different locations. The rough arrangement is similar: a horizontal laser sheet with the vertically mounted camera capturing images from below. However, for these experiments, a continuous wave Coherent Verdi V10 laser was used, mounted on an optical table on top of a rolling cart. As in the case of the streamlined body, 
the laser beam was converted to a flat sheet using a d-cylinder. In this case, the optics were also augmented by a set of two ninety degree mirrors to adjust the height of the sheet. The same particles were used to seed the tank as in the streamlined body experiments. The camera used to capture the particles lit by laser light, however, was a high-speed Phantom V10 camera with a $50 \mathrm{~mm}$ prime Nikon lens. The camera was again mounted below the tank, and a rough depiction of the field of view is shown in Figure 5-3. In the case of the flat plate, care needed to be taken in the positioning of the camera. Although ideally the sensors would be at the edge of the field of view in order to maximize the visualization of the fluid, this leads to an oblique view of the flat plate on which the sensors are mounted. The result is a ghost image of the sensor structure illuminated by the diffuse laser light which is present in all the images. This complicates the PIV calculations, as it is essentially a stationary image that contradicts information from moving particles. In order to reduce this effect, the camera needs to be position as close to the plane of the flat plate as possible. It is therefore a trade off between maximizing the field of view of the flow and maximizing the quality of the velocity approximation in the region very close to sensors. This trade off was improved as much as possible by reducing the amount of diffuse laser light. A certain amount of the laser sheet diffuses due to the particles, but the reflection of light off of the sensor structure was minimized by covering the areas in contact with the laser with black tape. The result allowed to the sensors to be offset from the center of the frame without substantial ghost images of the structure appearing. As in the previous case, the trigger signal for the camera was also captured in the pressure data acquisition, in order to provide a time reference between the PIV and pressure data.

\subsection{Data Processing}

\subsubsection{Pressure Data Pre-Processing}

In both experimental setups, the ambient electrical noise was of a low amplitude, as no external motors were running at the time of data collection. Therefore, the pressure measurements required little filtering. No low pass filtering beyond that necessary for antialiasing 
when downsampling was used on data for vortex tracking (when displaying data, it may be filtered for clarity).

As in the experiments discussed for tracking a cylinder, both sets of vortex tracking experiments were accomplished in a relatively small tank, leading to persistent standing wave oscillations in the free surface after the disturbances necessary to generate the vortices. These free surface oscillations are responsible for the common mode disturbance seen in figure 5-4 Although the pressure deviations due to surface waves decay with depth in linear wave theory, the large wavelength of the standing waves extends the influence. The expression for the dynamic pressure in linear wave theory is as follows [60]:

$$
P=\rho g \eta \frac{\cosh (k(h-d))}{\cosh (k h)}
$$

where $\rho$ is the density, $\eta$ is the surface deflection, $k$ is the wave number equal to two pi over the wavelength, $h$ is the full depth of the water, and $d$ is the depth of the sensors. Assuming a standing wave in the second harmonic lengthwise of the tank for the streamlined body experiments, which was observed in all cases, the static pressure due to the elevated surface is reduced by a factor of approximately one half. With this sort of factor, the 10 $\mathrm{Pa}$ amplitude oscillations observed in the pressure data would correspond to roughly $2 \mathrm{~mm}$ amplitude oscillations in the free surface, which matches well with observations. Despite the differing depths of the two sets of experiments, this measurement contamination was observed in both setups. The streamlined body experiments were more heavily affected, as expected by the shallower experiment depth.

In addition to the oscillations introduced by the surface waves, this noise source additionally complicates finding an accurate estimate for the far field pressure. Ideally the stable pressure measured before the vortices are generated is used as a reference for the pressure throughout the experiment, representing the pressure far from the influence of the vortices where the water is stationary. Unfortunately the oscillations due to the surface waves appear to have numerous frequency components, so identifying the mean pressure before vortex generation is subject to some error. Based on simple simulated results, an error of as little as $5 \mathrm{~Pa}$ is sufficient to significantly degrade estimation performance. 
The remedy for this noise source is the same as was implemented for the cylinder tracking experiments: considering the difference between adjacent pressure sensors. While the long wavelength of the surface waves are responsible for the contamination of the pressure measurements, they also allow for a straightforward solution. The wavelength is large in comparison to the array length, therefore all sensors are influenced in phase by nearly the same noise magnitude (See Figure 5-4). Using the inter-sensor pressure difference effectively removes any information about the mean pressure which both takes care of the common mode oscillations due to the free surface and the ambiguity of the pressure reference. In the case of the far field reference, it is only necessary to identify a common reference when there is no nearby stimulus. Therefore, it is sufficient to normalize the mean of several oscillations for each sensor when the vortices have not yet been generated or have long past. This solution is similar to the actual function of the biological lateral line, which measures pressure gradients through the canal system. Although there are many more factors at play in the biological system, such as the need to convert a flow velocity sensor into a pressure sensor using a system of canals, it is possible that the differential nature of the lateral line also addresses a similar problem in fish.

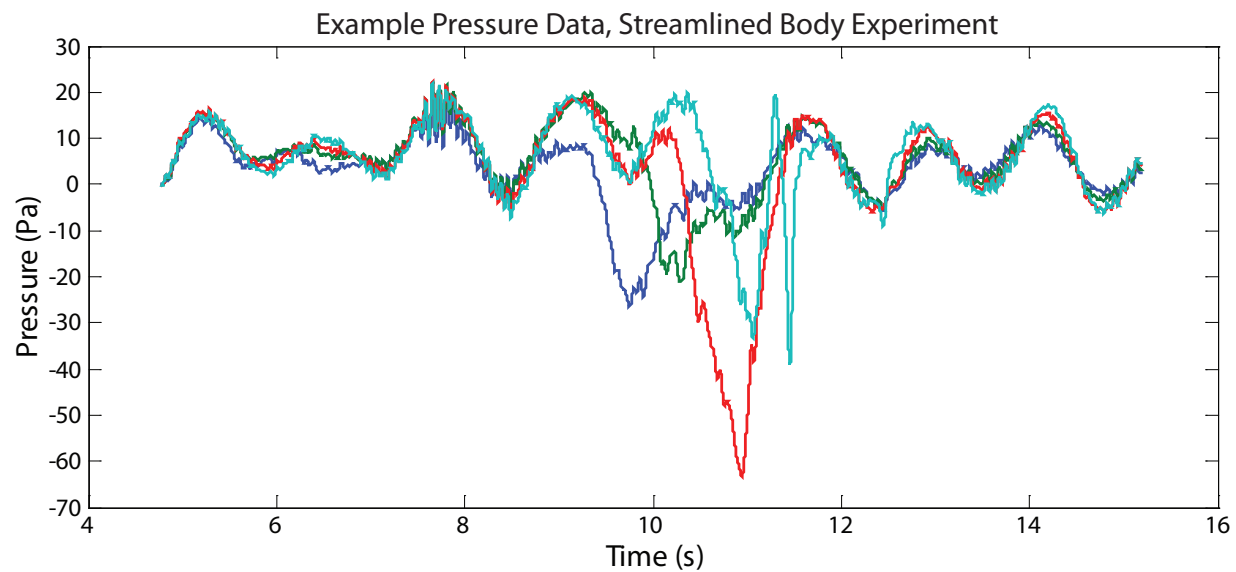

Figure 5-4: Typical pressure data set from streamlined body experiments. The surface wave effects are clearly visible. 


\subsubsection{PIV Processing}

A key element necessary for the use of the experiments to test vortex tracking elements is an independent estimate of the vortex position and strength. This is the primary reason for the particle image velocimetry used in conjunction with the experiments, as described in the experimental setup. In order to obtain estimates of the vortex states, the images of the particles are first processed to generate an estimate of the vector field in the imaged plane, and the vortices are subsequently located based on the velocity field.

For the PIV data gathered at the MIT towing tank, the data processing to obtain the velocity fields was done using DaVis v.7 software by LaVision, which corresponded to the PIV equipment in those experiments. The image files were lightly pre-processed prior to cross correlation, by masking the portion of the image in which the sensing body was visible. The cross correlation processing used the routines in DaVis based on a two dimensional fast Fourier transform. The final interrogation window size was 32x32 pixels. However, due to the large variety in the amplitude and direction of the fluid motion when vortices were present, the vector field was computed over multiple passes, starting with an interrogation window size of $64 \times 64$ pixels before the 32 pixel window, with two passes for each window size. This repetition was necessary in order to locate the correct region of the flow for cross correlation. No weighting was applied to the interrogation windows.

The PIV data gathered in the Singapore CENSAM tank, which corresponds to the flat plate sensor experiments, was processed in a very similar manner. However, the software used to process the images was Insight $3 \mathrm{G}$. The procedure for evaluating the velocity fields based on the particle images remained the same. First light pre-processing was done to mask out a rectangle enclosing the sensing body. Since the shape of the sensing body is also rectangular, this fit closely to the boundary of the structure. In processing the images, the interrogation window size was increased by one step for this experimental setup, due to a combination of a higher resolution camera and a larger distance from the image plane to the camera. The initial interrogation window size was $128 \times 128$ pixels, moving down to $64 \times 64$.

In both cases, a raw image was additionally kept with the processed PIV data, in order 
to obtain a physical reference. From these images, the location of an individual sensor port can be accurately identified, relating the spatial distribution of the velocity field to the coordinates in the pressure data estimates.

\subsubsection{Independent Vortex Identification}

In order to locate the vortices based on the velocity field generated by the PIV processing, an automated approach was developed and used. This approach was found to accurately identify vortices which had their core within the imaged area of the PIV (and therefore within the velocity field). At the same time, it also estimated the location of the second vortex which was generally outside the field of view. Unfortunately, the accuracy of the estimate of the second vortex could not be confirmed in all cases, but in the cases where the second vortex eventually entered the field of view, the results were consistent and smooth.

Besides the information provided on the vortex not captured in the PIV directly, the main motivation for using an approach based on an analytical model is the robustness to noise in the PIV vector field. Unfortunately, the nature of a pair of strong vortices in still water poses challenges for calculating the vector field, since there are important regions of both high and low velocity, and the direction of the vectors vary greatly. A certain degree of compromise is necessary between accurately estimating velocity near the center of the vortices and accurately capturing the flow field further away. The end result is that, particularly near the vortex core, it is slightly more likely for a vector to be the result of a faulty correlation. For direct methods in identifying a vortex from a vector field, only the vector field in a small area about the vortex is used, making it susceptible to the error of a single vector in that region. In contrast, fitting an analytical model makes use of the entire vector field for estimating each vortex, making it substantially more robust to errors in single vectors.

The automated approach works by fitting an analytical model to the velocity field calculated by PIV, similar to that described by Vollmers [81]. When applying an analytical model to the velocity field in order to identify the vortices, it is critical to use a model which accounts for the core. Although an inviscid, irrotational flow model is generally accurate 


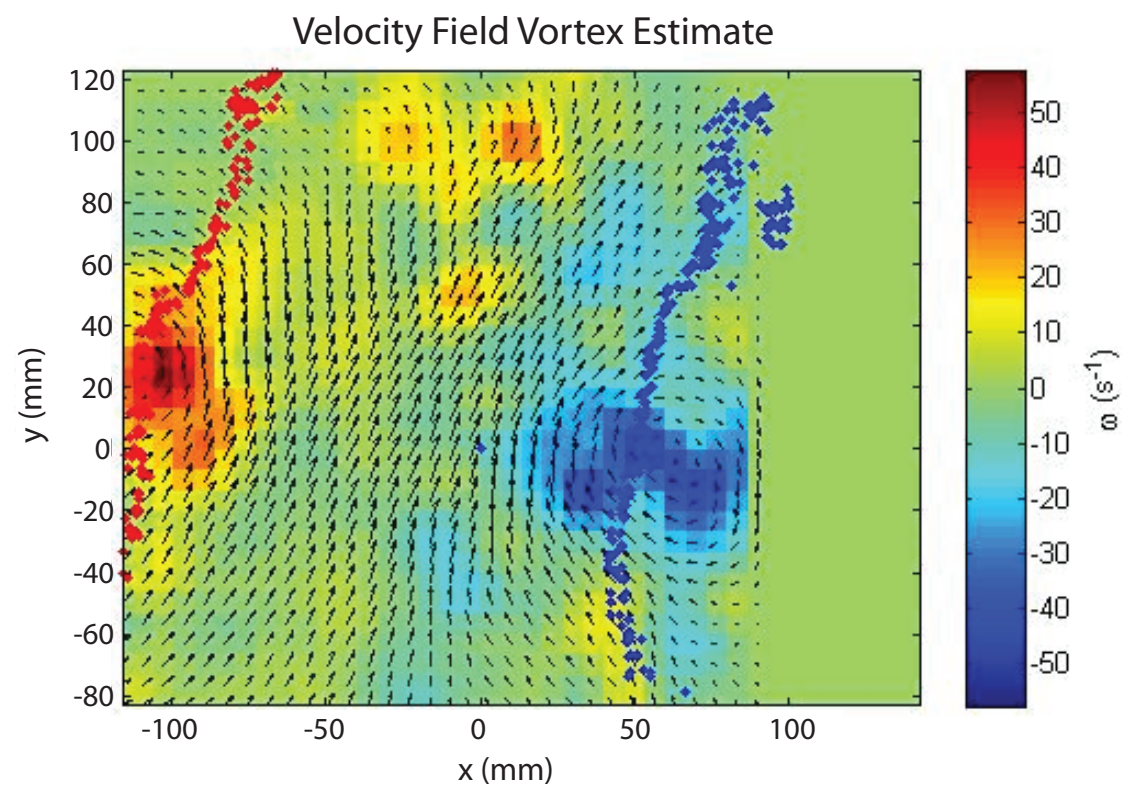

Figure 5-5: Depiction of the results using the optimization of an analytical model to identify the state of a vortex pair. The tracking results (solid colored dots) are overlaid on an intermediate frame and corresponding velocity field.

away from the vortex centers, viscosity plays a strong role at the center of the vortices. Without the core, the model dictates an unbounded velocity near the center of the vortex, which cannot match PIV data. Even though the discrepancy is localized, the large magnitude of the difference will influence the results. In this case, a Rankine vortex model was used, which models the vortex velocity field in two radially piece-wise regions:

$$
\begin{aligned}
V_{\text {tan }}(r) & =\frac{k}{r} \quad r>r_{o} \\
V_{\text {tan }}(r) & =\frac{k r}{r_{o}^{2}} \quad r<r_{o}
\end{aligned}
$$

Where $r_{o}$ refers the core size in the model, $k$ is the strength, and $V_{\tan }$ is the velocity tangent with respect to the center of the vortex.

The difficulty with defining a vortex core is that it requires specifying a radius. In order to avoid including another variable for optimization, a particular radius was assigned a priori for the vortices being fit to the velocity field. The radius,(FILL IN RADIUS), was chosen based on observations of the vorticity distribution in a few of the clearer PIV 
velocity fields. It was found that the vortex tracking results were not strongly dependent on the specified radius as long as it was within a reasonable range.

The actual optimization of the analytical model parameters is accomplished by minimizing a least squares cost function defined over the velocity field:

$$
J=\sum_{i}\left|\bar{V}_{i}^{\text {meas }}-\bar{V}_{i}^{\text {model }}\right|^{2}
$$

This cost function is based on the vector differences between the velocity field measured via PIV and the velocity field based on the analytical model for some combination of parameters. The optimization was implemented in Matlab using the gradient-based fmincon function, which also allowed the inclusion of a minimum y distance constraint. The only extra piece of information needed to run the algorithm is the initial guess for the vortex state. This was accomplished by manually picking the location of peak vorticity corresponding to the nearest vortex, in one of the first frames in which the circular streamlines are visible. The initial strength of this vortex was set to a unit magnitude with the correct sign. For the tow-tank experiments, the location of the second free vortex was chosen to have the same initial $X$ position as the primary visible one, and an initial spacing of 20 $\mathrm{cm}$ in the $Y$ direction. The strength of the second vortex was fixed to be equal in magnitude but opposite in sign to the near vortex. This was found to be necessary not just as an initialization for the algorithm, but throughout in order to maintain a smoothly varying estimate for the second vortex. In the case of the Singapore experiments, where the second vortex is an image, only one vortex needed initialization. At each timestep, the initial vortex state was used to begin the least squares optimization, and the result was propagated by one timestep to generate the initialization values for the next PIV frame. This approach generated consistent results that were robust to reasonable changes in the initialization.

The automated approach for extracting and independent estimate of the vortex state from the PIV was validated against more direct traditional methods for this particular application. In both sets of experiments with vortices, the vortices were generated by the motion of a foil or plate sweeping an arc, but the imaged area was substantially apart from the foil. This means that in the imaged area, the flow was dominated by a few large vortices, 


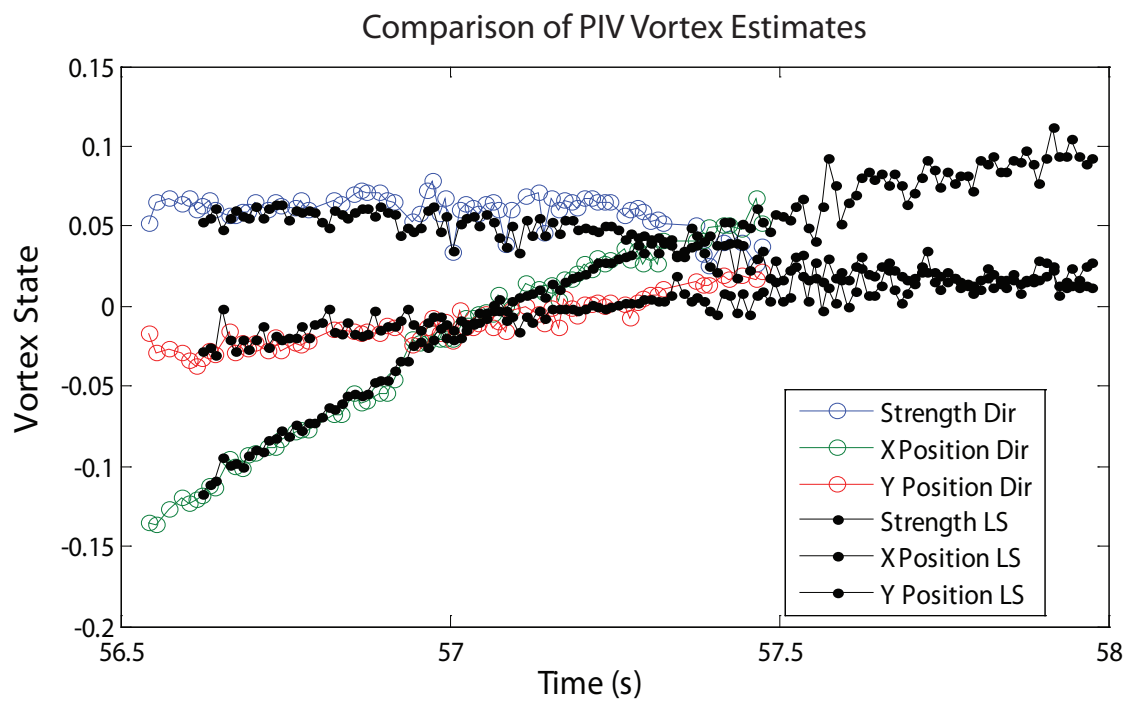

Figure 5-6: Comparison of the estimated state of a vortex based on PIV vector fields. Results for the vortex closest to the sensor body of a vortex pair are shown. In black filled dots are the estimates based on a least-squares fit using an analytical vortex pair model. In open colored circles are directly identified estimates based on the location of the peak vorticity and area vorticity integrals.

and very little shear flow. Given this situation, the local maximum in vorticity magnitude is a good measure of the location of a vortex [39]. A comparison of the $X$ and $Y$ position of the vortex estimated via the maximum in vorticity against the analytic model fitting is shown in Figure 5-6. The maximum vorticity only provides an estimate when the center of the vortex is within the image. Over that region, the two estimation methods agree considerably, with the algorithm based on the analytical model having slightly less variation from image to image, as might be expected since many more vectors are involved in the estimate.

Estimating the vortex strength directly from the PIV vector field for comparison was accomplished using both a line integral and a surface integral to compute the circulation over a region encompassing the vortex core.

$$
\Gamma=\int_{C} \bar{v} \cdot d \bar{s}=\iint_{A} \bar{w} \cdot \hat{n} d A
$$

where $A$ is a surface enclosing the vortex in the two dimensional PIV plane, and $C$ is the 
corresponding closed boundary to that surface. $d \bar{s}$ is a differential length element along the boundary of the surface, and $\bar{w}$ is the vorticity. Both approaches were implemented using a rectangular region centered about the local extremum in vorticity. Although a single integral was used in each case, several sizes were tested in order to find the maximum circulation. The maximum circulation was taken to be an accurate representation of the true vortex circulation, as the inclusion of the vortex core would reduce it if the region is too small, and other vortices or fluid interactions would reduce it if the area is too large. Both integral methods were found to provide nearly identical results when applied in this manner. In comparison, the analytical model method (Figure 5-6) matches the direct integral method results reasonably well in vortex strength. Unlike the comparison in position however, the analytical model is consistently below the direct method estimate during the later portion of the data set. It is unclear which of the two approaches is in error. However, given the reduced information used to calculate the direct approximation, it is the more likely option.

Overall, automatically fitting the analytical model to the PIV velocity fields generates consistent estimates of the location and strength of the nearest vortex. As shown, this estimate also matches well with local direct estimates of the strength and position of the vortex. There is also a slight improvement in the estimate consistency from one time-step to the next for the analytical model compared to the direct methods. The key point however, is that the automated PIV estimates based on fitting an analytic model generally agree very closely to the direct approach which assumes no model. Therefore, there is no concern that the line vortex model used as the analytical model for the PIV will generate results that are favorably inclined to the estimates based on pressure data, regardless of similarities in the analytical model.

\subsection{Real Time Vortex Tracking}

In order to solve the inverse problem of tracking a vortex in real time based on the data provided by an artificial lateral line, several components must be addressed. First, an accurate forward model is needed to predict the pressure measurements based on the state of a vortex (or vortices). Second, depending on the characteristics of the forward model, 
an appropriate estimation technique needs to be identified. Using the estimation technique and the forward model together, the results can be optimized for the problem and the results compared to the true vortex state directly based on the PIV data.

It is important to recognize at the outset that the problem of tracking a vortex based on pressure measurements is ill-posed when one considers a certain maximum of precision or noise in the measurements. This can be most clearly seen in an extreme case where the pressure sensors all measure the same reference pressure, there is no unique vortex state that corresponds to it. For example, it could be that there are no vortices present in the fluid, or it could be that there is a vortex, or many vortices, but that they are all too far away to affect the sensors significantly in comparison to the noise floor. This issue of range is well known for the lateral line, since near field pressure disturbances decay quickly with distance. The implication on the inverse problem is that there will be certain types of measurements scenarios in which tracking a vortex will be impossible, and others in which certain aspects of the vortex are ambiguous. This is considered in more detail later in section 5.5.

\subsubsection{Experimental Vortex Forward Models}

This section addresses the question of identifying a good forward model which relates the vortex states of interest to the pressure measurements. Due to the differences in the flow for the two experimental setups (streamlined body and flat plate body), different forward models will be needed. The main measure of quality for a forward model is the ability to predict the measured pressure given the known relevant vortex states. This will be evaluated using the vortex states estimated from the PIV data and compared against the experimentally measured pressure for a representative data set. However, since we are interested in solving the inverse problem, we also desire a simple forward model with the fewest number of parameters. Excessive parameters describing the flow that do not substantially alter the pressure measurements will be very difficult to estimate from the pressure measurements due to the low sensitivity. 


\section{Streamlined Body Model}

Based on the observed trajectory of the vortices in the experiments and the success of the analytic models fit to the PIV data, there is already a sense of the appropriate analytical model for characterizing the velocity field due to a small number of vortices. In the case of the experiments with the streamlined body, there are two vortices present near the sensors. As discussed previously, the fact that the two vortices generally travel parallel to the sensor body after being generated implies that there is little influence on the vortices from the sensing body and therefore the two dimensional approximation should not have a wall or any image vortices. As a result, the forward model will effectively use ghost sensors, calculating the pressure in the flow at the correct locations, but without any effect on the flow. In particular, this means that the flow at those points will not be parallel to the sensor array. However, this violation of the boundary conditions is acceptable because this is a two dimensional approximation to the three dimensional flow.
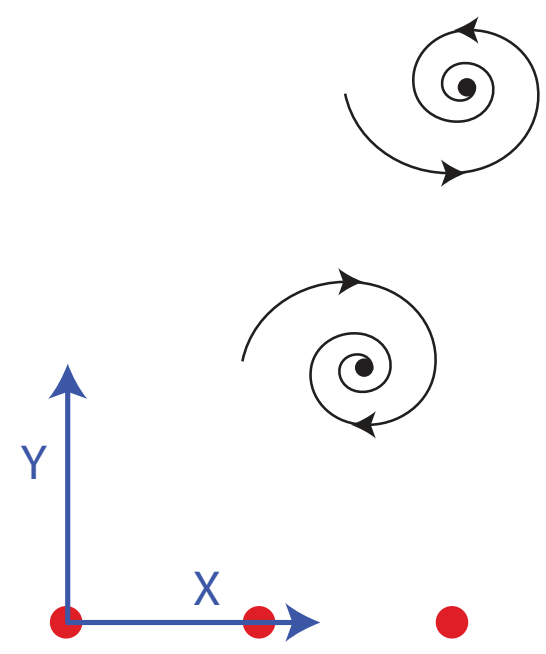

Figure 5-7: A general model for the two vortices near the streamlined body.

A complete model for two vortices (pictured in Figure 5-7) would include a full state for each of the vortices consisting of an $x$ position (parallel to sensor array), $y$ position (perpendicular to sensor array), and strength. This is a closer approximation to the true experimental scenario. The velocity in this case would be determined by the interaction between the two vortices. However, there is still the question of removing parameters with 
low sensitivity. One simple reduction in the parameters is immediately apparent given the particular experimental setup here. Since the vortex pair stimulus consists of the starting and stopping vortices of a foil, the strength of these vortices should be equal in magnitude but opposite in sign. This assumption will be used throughout whenever a vortex pair is being considered.

The forward model consists of two components. The primary interest is in the relationship between the vortex pair state and the pressure, but there is also a component of the model that describes the state progression. For the current description of the vortex pair, the state vector would consist of five variables: one strength and four positions. Along with the boundary conditions discussed, this is sufficient to define the complex potential describing the flow:

$$
\Phi(z)=-i k \log \left(z-z_{1}\right)+i k \log \left(z-z_{2}\right)
$$

where $z_{1}$ and $z_{2}$ are the complex representations of the center for each vortex. The induced velocity that governs the motion of the vortices can be calculated by considering the velocity (derivative of the complex potential with respect to $\mathrm{z}$ ) at the center of one of the vortices with only the other one acting. The result is $u-i v=\frac{i k}{z_{1}-z_{2}}$. As a result, the description of the state progression is fairly straightforward. We assume that the vortex strengths do not decay (on the timescale of the experiment), and that the first derivative of the position is given by the induced velocity just described.

The second part of the forward model with unconstrained position relates the vortex pair state at a time instant to the pressure. This is derived directly from the unsteady Bernoulli equation [3]:

$$
\begin{aligned}
P(z)-P_{\infty}= & -\frac{1}{2} \rho\left|\frac{\partial \Phi(z)}{\partial z}\right|^{2}-\rho \frac{\partial \Re(\Phi(z))}{\partial t} \\
P(s)-P_{\infty}= & -\frac{1}{2} \rho\left|\frac{-i k}{s-z_{1}}+\frac{i k}{s-z_{2}}\right|^{2}+\rho \frac{k y_{1}}{\left(s-x_{1}\right)^{2}+y_{1}^{2}} u+\ldots \\
& -\rho \frac{k y_{2}}{\left(s-x_{2}\right)^{2}+y_{2}^{2}} u+\rho \frac{k}{\left(s-x_{1}\right)^{2}+y_{1}^{2}} v-\rho \frac{k}{\left(s-x_{2}\right)^{2}+y_{2}^{2}} v
\end{aligned}
$$


Here we make use of the fact that the pressure sensors are defined on the $x$ axis and so have no imaginary component. The position of the sensors is therefore given by the real scalar $s$. Also, note that the real part of the complex potential is the angle that the point of evaluation makes with the center of each vortex, scaled by the vortex strength. This model for the pressure uses the vortex state at one time instant only, since it makes use of the instantaneous estimate of the vortex velocities based on the their relative position. For brevity, this model will be referred to as the 1.6 model, as it has one vortex entirely specified, and two thirds of the parameters specified for the second vortex.

A second parameter which should be considered for removal is the relative $x$ position of the second (more distant) vortex. Given the direction of motion, the separation in the $y$ direction is critical to the two-vortex model since it produces the velocity in the $x$ direction. However, the vortex pair generally have a very small velocity component in the $y$ direction, and therefore the $x$ position of the two vortices should be very similar. This is confirmed via the estimates of the vortex locations provided by the PIV. Therefore, one logical simplification would be to assume that the $x$ positions of the two vortices are equal. This model is very similar to the one described above, except that the velocity component $v=0$ for both the state progression and the pressure equation (5.3), as well as $x_{1}=x_{2}$. Since this model effectively has one and a third of the parameters of a single vortex (without velocity specified), it will be referred to in brief as the 1.3 vortex model.

Another possibility in modeling the flow near the streamlined body, for the purposes of predicting the pressure, is to focus on the closest vortex only. Due to the proximity of this vortex, it will cause the majority of the contribution to the pressure measurements. For a single vortex, the vortex state under the two dimensonal constraint being used in this chapter can be fully defined with three parameters: two for the position of the vortex and one for the strength or circulation. However, a single vortex would be stationary in still water without an image vortex due to interactions with nearby bodies or fluid structures. Clearly this is not the case in either of the experimental setups (as observed in the PIV results). Therefore, if we approximate the scenario by a single vortex in this situation, the state needs to be augmented by a velocity. For the general experimental setup in this chapter, the primary direction of motion is along the axis of the sensor array, so only one 
velocity component will be used: $U$ along the $x$ axis. In this model, the state progression is defined by a constant vortex strength and $y$ position, and with the derivative of the $x$ position equal to $U$. The equation of pressure is given as follows:

$$
P(s)-P_{\infty}=-\frac{1}{2} \rho\left|\frac{-i k}{s-z_{1}}\right|^{2}+\rho \frac{k y_{1}}{\left(s-x_{1}\right)^{2}+y_{1}^{2}} U
$$

To test the accuracy of these three forward models, they are used to predict the pressure in an experimental setting given a vortex trajectory that is extracted from the corresponding PIV. The results of this comparison for representative data set which had a good, complete set of PIV-extracted states is displayed in Figure 5-8. In examining the first two parts of this figure, which correspond to the 1.3 and 1.6 vortex models, it is clear that there are very strong similarities and that they capture a substantial portion of the actual measured pressure. Both the 1.3 and 1.6 models capture the negative pressure peak at the tail end of the time series. At the beginning of the time series, the 1.3 model which assumes the $\mathrm{x}$ position of the two vortices are equal, is more consistent in recreating the pressure in the first large negative pressure peak. In between these two large peaks, the pressure recreation is similar for both models, and underestimates the actual measured pressure for some of the sensors, while matching the others. One substantial difference between these two models is that the 1.6 model more readily recreates pressure greater than the reference. Looking at the results of the single vortex model, where the velocity $U$ was estimated based on the smoothed derivative of the $x$ position extracted from the PIV data, the pressure is generally heavily over estimated in magnitude. This is particularly true in the later parts of the time series.

The reconstruction of the pressure is improved when considering the difference between adjacent sensors (Figure 5-9). As discussed earlier, this removes the effect of a drifting reference pressure, primarily the effect of small amplitude, large wavelength, oscillations in the free surface. Based on this figure, it is even clearer that the two vortex models outperform the single vortex model, and that there is no obvious benefit to allowing the second vortex to differ in the $x$ position from the first. As a result, we shall identify the 1.3 vortex model as the most appropriate forward model for solving the inverse problem for 

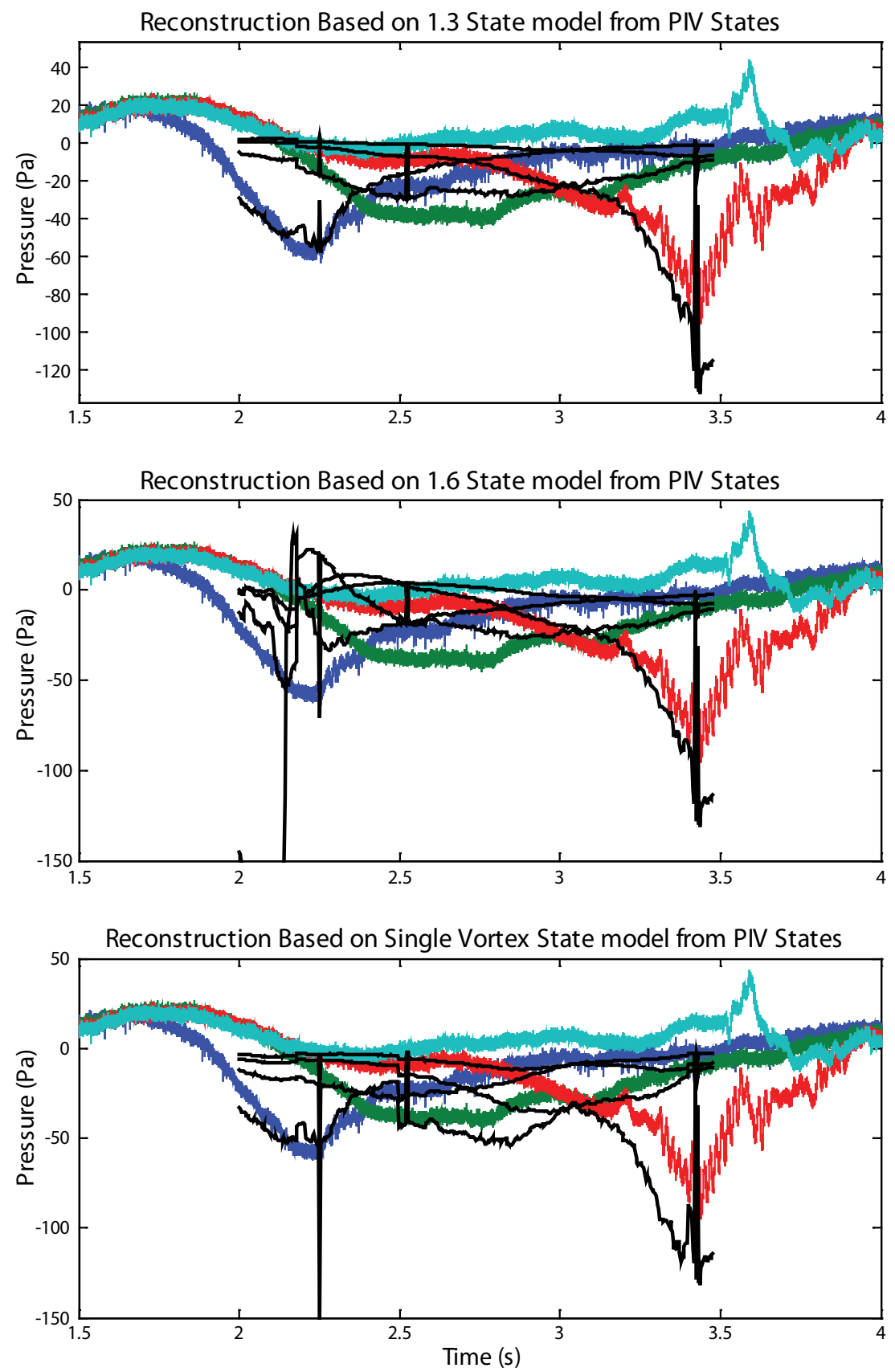

Figure 5-8: Reconstructed pressure from different forward models based on PIV vortex states for streamlined body experiments. The raw measured pressure is plotted in color. The thin black lines represent the reconstructed pressure predicted by the forward models. 

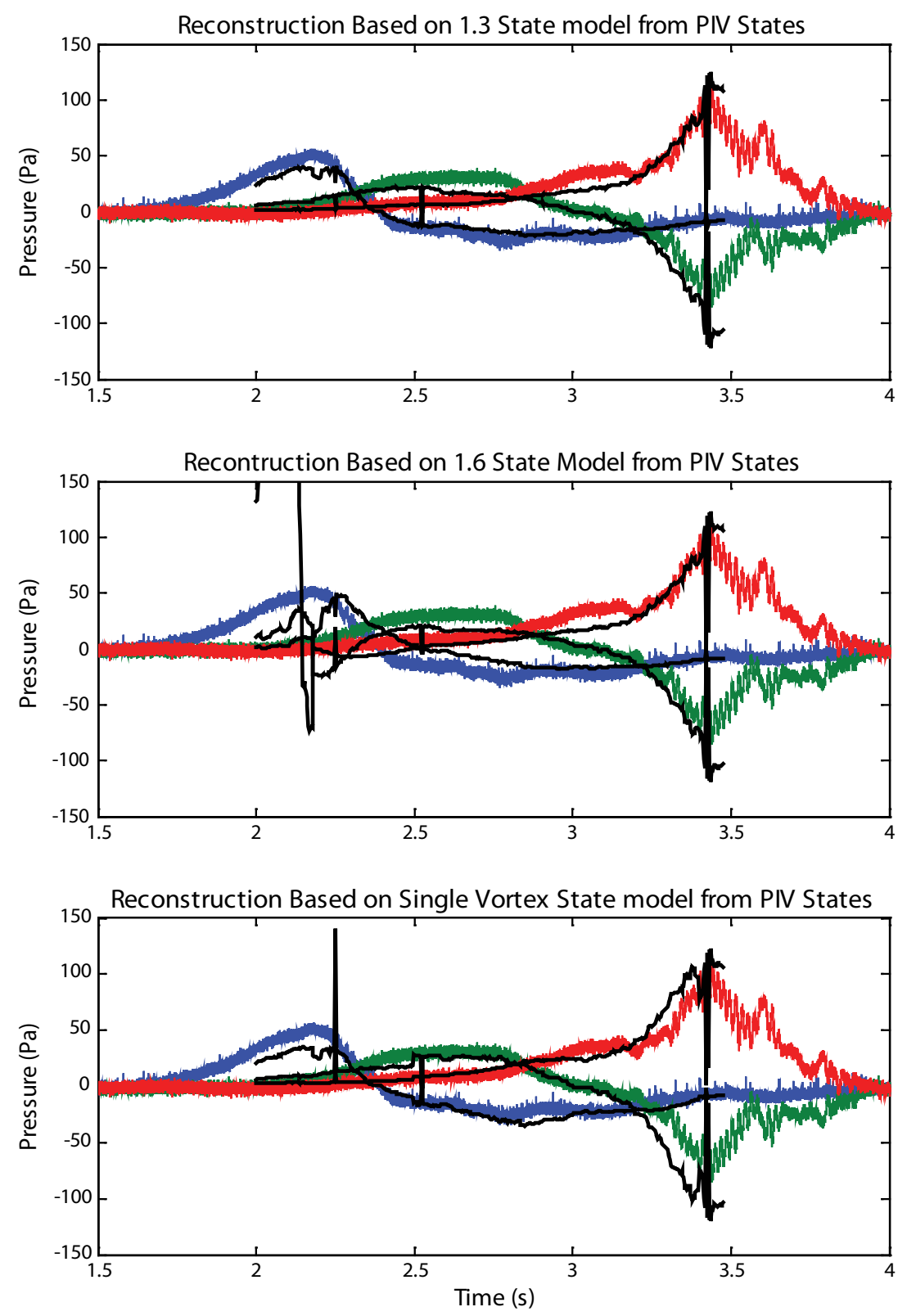

Figure 5-9: Reconstructed pressure differences from different forward models based on PIV vortex states for streamlined body experiments. The raw measured pressure is plotted in color. The thin black lines represent the reconstructed pressure predicted by the forward models. 
pressure measurements on the streamlined body.

\section{Flat Plate Body}

In the case of the experimental setup with the sensor array embedded in a flat plate, there are clear indications of interaction between the vortices in the fluid and the sensor structure. In fact, the majority of the data from these experiments corresponds to time when there is only one vortex in front of the sensors, and it is moving due to the interaction with an image vortex generated by the presence of the flat plate.
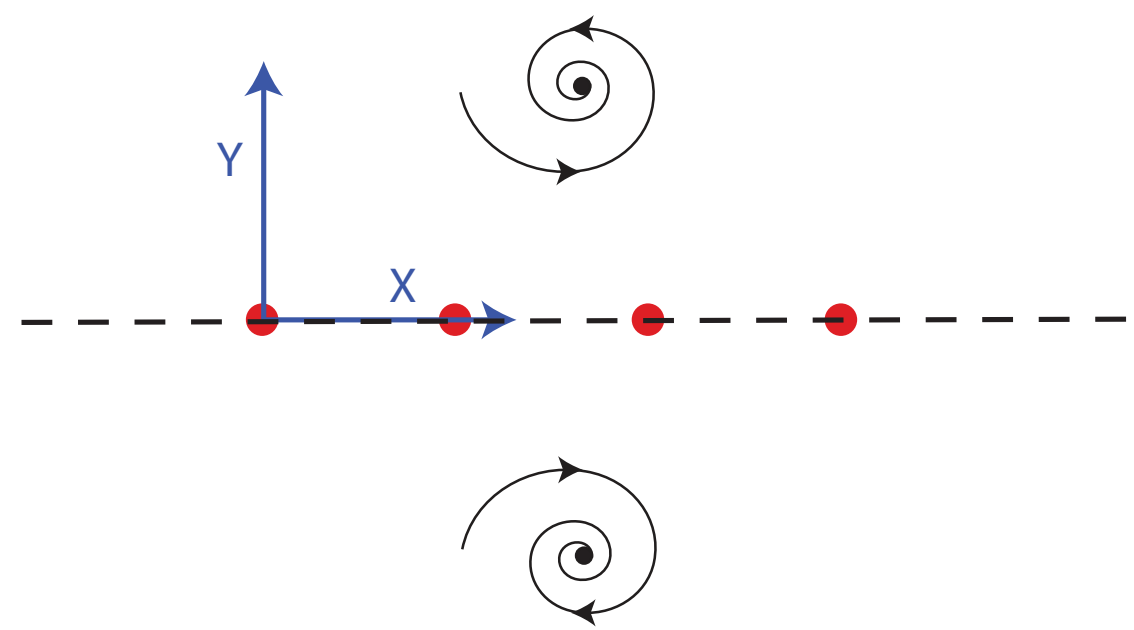

Figure 5-10: A general model for a single vortex near sensors mounted on a flat plate. The dotted line represents the plane of the plate.

For this time when only a single vortex is near the sensors (after the second vortex in the original pair as moved away from the sensors), the model for the flow is fairly clear. There are much fewer options in terms of choosing a forward model when compared to the streamlined body case. First of all, in the case of the flat plate, the two dimensional approximation to the flow is much more accurate. The boundary conditions on the flat plate can be satisfied in two dimensions using an image vortex, though this extends the flat plate infinitely to either side. The diagram for this model setup is displayed in Figure 5-10 
The corresponding complex potential for this flow model is given by:

$$
\Phi(z)=-i k \log \left(z-z_{o}\right)+i k \log \left(z-\overline{z_{o}}\right)
$$

where $z_{o}$ is the position of the single vortex and $\overline{z_{o}}$ is its complex conjugate, representing the position of the image vortex reflected over the surface of the flat plate. In this case, only three state parameters are needed to define the state vector: the strength of the vortex and the two components of the position in the plane. The induced velocity due to the image vortex results in only a component along the $x$ direction, equal to $u=\frac{k}{2 y_{o}}$. The pressure can again be found via the unsteady Bernoulli equation:

$$
\begin{aligned}
P(z)-P_{\infty} & =-\frac{1}{2} \rho\left(\frac{\partial \Phi(z)}{\partial z}\right)^{2}-\rho \frac{\partial \Phi(z)}{\partial x_{o}} \frac{\partial x_{o}}{\partial t} \\
P(s)-P_{\infty} & =-\rho \frac{2 k^{2} y_{o}^{2}}{\left(\left(s-x_{o}\right)^{2}+y_{o}^{2}\right)^{2}}+\rho \frac{k^{2}}{\left(s-x_{o}\right)^{2}+y_{o}^{2}} \\
& =\rho k^{2} \frac{\left(s-x_{o}\right)^{2}-y_{o}^{2}}{\left(\left(s-x_{o}\right)^{2}+y_{o}^{2}\right)^{2}}
\end{aligned}
$$

In this case, the alignment of the pressure sensors with the boundary condition along the $x$ axis results in a number of simplifications taken into account in the brief derivation. These simplifications stem from the fact that the flow velocity at a point on the $x$ axis will only have a component along the $x$ axis due to symmetry. Therefore, the velocity from the derivative of the complex potential will be a real quantity, as shown. In addition, since $x$ axis is a streamline, the stream function is constant along it, implying that the imaginary component of the complex potential is similarly constant. As a result, the time derivative term in the Bernoulli equation can be treated with respect to the complex potential instead of the velocity potential, for the sake of simplicity. This was not possible in the streamlined body case.

Although the choice of a forward model is fairly clear in this scenario, it is still useful to compare the predicted pressure based on the PIV state history to the actual measured pressure. Figure 5-11 demonstrates the result for one representative data set. In this case, the pressure data is of small amplitude, and the absolute pressure contains large mean 
pressure shifts due to surface oscillations, so only the pressure difference is considered. The match between the reconstructed pressure and the measured pressure is very good. At the beginning of the time history, the second physical vortex has a significant impact on the measured pressure which cannot be accounted for by the single physical vortex in the forward model. After approximately the 17 second mark, however, the reconstructed pressure difference matches the measured pressure difference in both shape and magnitude. There is a slight deviation again at the tail end of the data, where the vortex appears to stall in the PIV. It is unclear if this is due to end effects of the flat plate not being modeled or to the incorrect extraction of the state of a very weak vortex from the PIV data. The strength of the vortices in the flat plate experiments decay substantially more than in the experiments with the streamlined body. Regardless of the differences at the start and end of the file, the close match between the reconstructed and measured pressure through the majority of the data implies that the reflected vortex model is a good forward model for the vortex experiments with the sensors embedded in a flat plate.

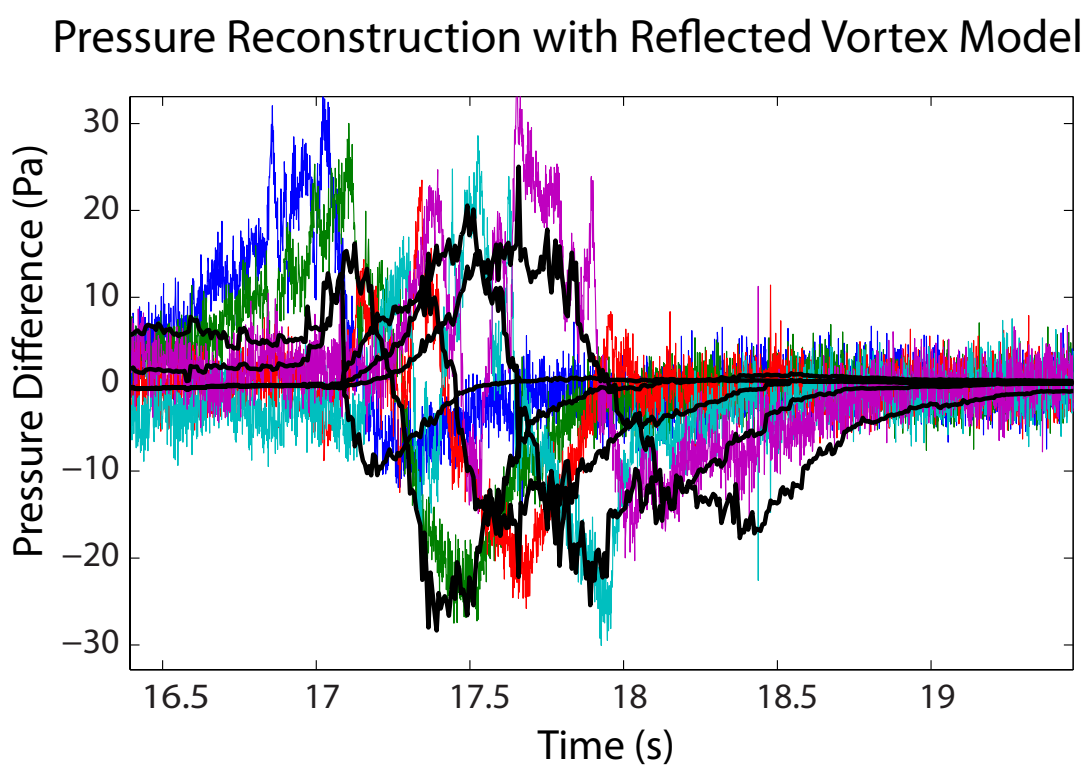

Figure 5-11: Reconstructed pressure differences from a forward model with a single vortex and its image based on PIV vortex states for the flat plate body experiments. The raw measured pressure is plotted in color. The thin black lines represent the reconstructed pressure predicted by the forward model. 


\subsubsection{Estimation Methods}

The goal of the experiments and the identification of a forward model is to be able to solve the inverse problem and verify that it has been accurately resolved. In order to tackle the inverse problem, the primary requirements are to be robust to noise and changes in the model while using as much of the data history as possible. While it is possible to attempt to invert the pressure measurements separately for each time instant, this would guarantee a sub-optimal result. For example, the pressure described by equation (5.8) is a function of the strength squared only, and therefore the direction of rotation of a vortex would be impossible to determine from the measurements at a single time instant. However, the rotation of the vortex also determines the direction it moves, resolving the ambiguity if data across multiple time instances is used. In addition, the solutions to the inverse problem need to be limited to certain conditions, implicitly or explicitly, since the inverse problem is technically ill-posed under certain conditions (for example, small signals).

Similarly to Chapter 3, the problem as it has been described for tracking a vortex via pressure sensors naturally fits into the hidden Markov model structure where the measurements only depend on the current vortex state, and a new vortex state depends only on the one directly before it. The actual model for the vortex progression is continuous in time as opposed to the discrete time description just given, but the HMM concept can be extended. This model structure makes it possible to use a number of estimation algorithms, including the Kalman and particle filters among others. Both the Kalman and particle filters satisfy the desired characteristics for an estimation algorithm.

\section{Estimation Algorithm \& Implementation}

Among the different possibilities for an estimation technique, the Kalman filter was chosen for this problem due to its ability to tune the length of the memory of the process and its widespread application to many problems in engineering. Restricting the length of the memory of the Kalman filter allows the estimate to change if the model changes or drifts due to external influences. For example, in the case of a single vortex that can only move in the $x$ direction, the limited memory allows the $y$ position to change even after a substantial 
amount of time has passed with one $y$ position. As a result of its wide use, many different variations of the original Kalman filter are available for different degrees of nonlinearity and different types of models. As is evident from equations (5.3) and (5.8), the equations relating the vortex state vectors to the vector of pressure measurements are not linear. Multiple approaches are known to apply the Kalman filter to nonlinear problems $[41,27]$. In this case, a simple first order approximation of the nonlinear pressure function is used via the extended Kalman filter algorithm. In addition, the continuous time state progression was coupled with a discrete time measurement update. The details of the implementation are described in [27].

The Kalman filter and it's nonlinear variants, including the extended Kalman filter being used in this chapter, model the noise in the system as being white and having a Gaussian distribution. The Gaussian distribution is not a concern in tracking vortices as it was in Chapter 3 for tracking a cylinder. The measurement noise is reasonably described by a Gaussian distribution already. However, modeling noise in the state transition model as Gaussian is an approximation, since this noise term stands in for a number of effects that are not included in the flow model such as other vortices or any external flow. The state transition noise terms also play an important role in the convergence and adaptation of the Kalman filter algorithm (see next section). The vortex tracking problem differs significantly from the cylinder tracking problem in that there is a natural boundary that corresponds to the physical sensor body. Specifically, if the vortex strength estimate is not zero, then the pressure corresponding to an estimate in which the $y$ position is very small will be very large and therefore substantially different from the measured pressure. This large increase in error as the vortex approaches to close to the sensors acts to repel the estimate from having the vortex cross behind the sensing plane (leading to a non-physical situation). There are two exceptions in which it is still possible to have the vortex estimate cross the $x$ axis. First, if the vortex estimate places the vortex far from the sensors in the $x$ direction, then the cost of crossing the sensor array is strongly reduced. Second, during the initial stages of the Kalman filter, the magnitude of the state estimate corrections is large to compensate for the lack of information, which may lead to crossing the $x$ axis without evaluating an intermediate point with small $y$ position. These possible exceptions both 
relate to the initialization of the Kalman filter. By not initializing the estimation algorithm before the sensor array registered a signal from the vortices, and by limiting the magnitude of the initialization of the estimate error covariance matrix, the issue of a vortex crossing behind the sensor array plane was non-existent in practice.

\section{Parameter Determination}

The extended Kalman filter has a number of internal parameters that need to be specified. These include the initial state estimate covariance matrix, the transition covariance matrix, and the measurement covariance matrix. As a general rule, values for these internal knobs in the extended Kalman filter were kept the same for all the vortex tracking experiments, regardless of whether streamlined body or flat plate was used to measure the pressure. This was done in order to keep from overtuning the estimation method to each data set. Due to the different state vectors in the two cases, there are still slight differences. The result is a rough general set of covariance matrices for estimating vortices.

The first of these internal parameters to consider is the initial state covariance matrix. The Kalman filter produces real time estimates of the distribution of the state vector (assuming a Gaussian form), which corresponds to the mean value of the state and its error covariance matrix. The confidence in the state estimate, represented by the covariance matrix, is instrumental in determining the magnitude of the correction applied in response to discrepancies between the measured and predicted pressure at the next measurement update. In all of the experiments in tracking vortices, little information is used to provide the initial state value, and therefore there is almost no confidence in it. It is preferable that the state estimate be heavily corrected once the first measurements are used. For these reasons, the initial values of the state covariance matrix need to be fairly large. In addition, since there is no a priori reason to introduce dependence between the variables, the initial state error covariance is diagonal. The actual values of the diagonal elements were chosen based on the dimensions of the variables and the problem. All the length variables in the state were initialized to have a variance of $(0.1 \mathrm{~m})^{2}$. The strength variable in the state vector has units of $\mathrm{m}^{2} / \mathrm{s}$. Given the fundamental length scale of the problem is in $\mathrm{mm}$, and that a typical value for the strength is $0.01 \mathrm{~m}^{2} / \mathrm{s}$, the initial variance was set to $1 \mathrm{e}-5\left(\mathrm{~m}^{2} / \mathrm{s}\right)^{2}$. 
The choice of the measurement and state transition noise covariance matrices are interrelated as they strongly affect the behavior of the Kalman filter. In particular, the relative magnitudes of the two matrices determines the reliance of the algorithm on the new measured information versus the previously estimated state. If the measurement noise variance is small with respect to the state transition noise variance, then the estimate of the state will approach the instantaneous inverse estimate of the state given the measurements. If the measurements still have substantial noise, this means that the state estimate will vary wildly. The extreme case of zero measurement noise is not valid for the variants of the Kalman filter. In the opposing situation, when the state transition noise variance is very small with respect to the measurement noise variance, the state estimate is much slower to respond to discrepancies between the predicted and measured pressure. It is possible to have zero transition noise variance, implying that the state transition is known exactly. This typically leads to a "hardening" of the state estimate where, after a certain amount of time in which the transients due to the initial state error covariance die out, the state estimate may progress via the state transition rules only and be completely independent of new measurements. In this context, one effective way to think of the state transition noise variance is as a lower bound to the state error covariance. By upper bounding the confidence in the state estimate, the algorithm is always able to change its estimate based on new information. For this reason, it is frequently beneficial to model some noise in the state transition, even if there is no physical source for the noise.

The ratio of the measurement noise variance to the process (or state transition) noise variance was chosen based on a combination of experimentation and relative signal magnitude. The actual measurement noise corresponding to the Honeywell pressure sensors being used is very small, with a standard deviation of just a few Pascals. For the state transition, there is no noise of the same sort which is easily quantifiable, but it is possible to examine the amount of drift in the $y$ position component from the PIV estimate of the position. This can be about $1 \mathrm{~cm}$ over the entire data set, and closer to $1 \mathrm{~mm}$ on a more local scale. This is sufficient to give a rough magnitude level for each of the covariance matrices, with the measurement covariance having elements with magnitude of $10 P a^{2}$ and the state transition covariance having elements with magnitude on the order of $10^{-} 6 \mathrm{~m}^{2}$. 

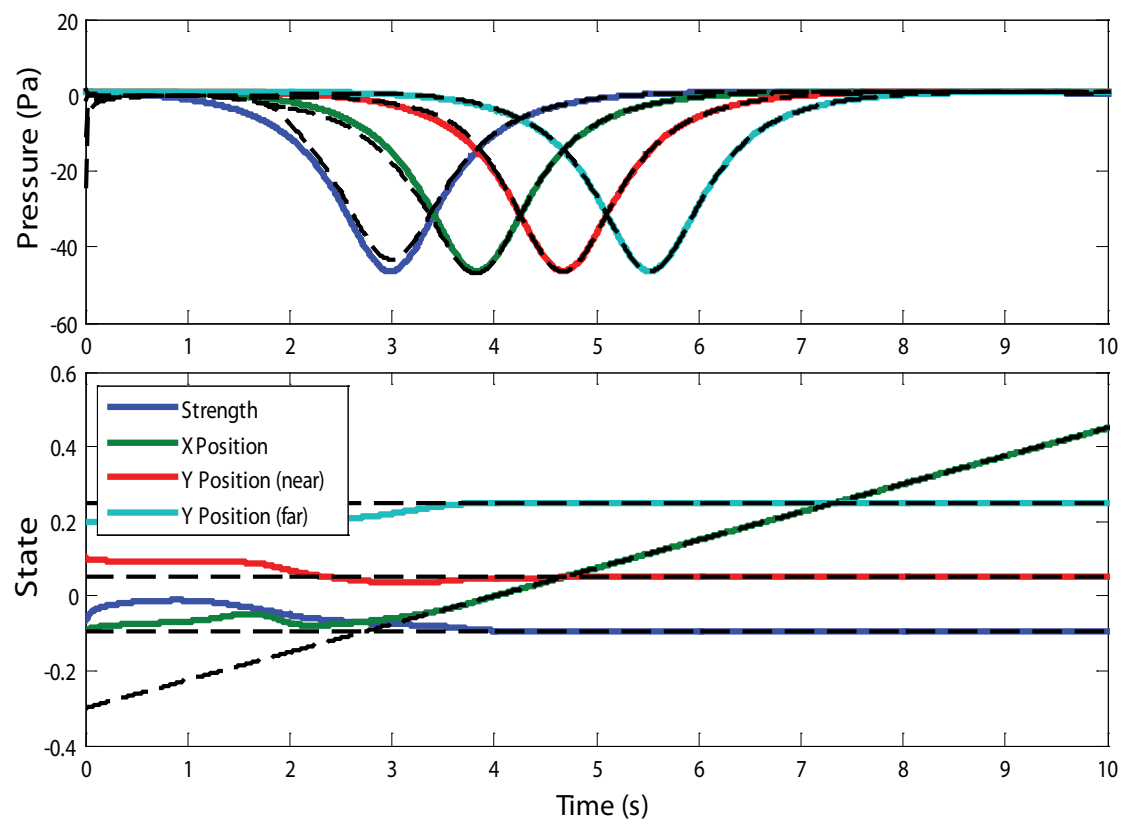

Figure 5-12: Simulated extended Kalman filter application for tuning internal parameters. The upper subfigure plots the simulated pressure (dotted black line) and the reconstructed pressure (color) based on the Kalman filter state estimate. The lower subfigure displays the convergence of the state estimate (color) to the true state (dotted black line).

The rough initial values for the standard deviation corresponding to each noise covariance matrix were then tuned further by testing the extended Kalman filter algorithm against artificially generated data. In particular, this addressed the question of relative levels within each covariance matrix. For the measurement covariance matrix, each sensor acts independently and has independent noise. Therefore it is a small assumption to represent the measurement covariance matrix as a diagonal matrix with equal elements along the diagonal. However, in the case of the state transition noise covariance, it is likely that the elements of the matrix will not all be the same. This is where the simulated experiments come in use. For these, pressure measurements were generated using the forward models discussed earlier, with the vortices following simple trajectories according to the induced velocity from the model. Using an initial condition sufficiently removed from the true state, different values for the state transition covariance matrix could be tested and the speed of convergence to the true state (as well as the final error) be used as a metric. Figure 5-12 shows the simulated pressure and convergence results for an example based on 
the streamlined body forward model. The state transition covariance matrix was assumed to be diagonal, as each state variable can vary independently. Based on experiments with these simulated trials, it was found that the variance of the strength should be smaller than that of the position variables, as expected based on their relative dimensions, but only by a factor of 100. Differences between the $x$ and $y$ position variance appeared to have had minor effects. However, in the case of the 1.3 vortex streamlined body forward model, the distance of the second vortex does not affect the measured pressure as strongly. Small changes in the $y$ location of the second distant vortex do not cause changes in the pressure of the same magnitude as small changes in the $y$ or $x$ location of the nearest vortex. This reduced sensitivity also means that the Kalman filter is slower to correct this state variable. In order to counter this, increasing the state transition variance for this state variable reduces the confidence in the estimate of this relatively insensitive variable, leading to faster convergence. Increasing the variance by a factor of 5 relative to the $x$ and $y$ position of the nearest vortex generated good results.

In running the extended Kalman filter implementation on the experimental data, it was found that the optimal ratio of the measurement to process covariance changed slightly given the magnitude of the pressure signals. For large magnitude pressure signals with peaks of up to $100 \mathrm{~Pa}$, a measurement noise standard deviation of $10 \mathrm{~Pa}$ would work well, while a variance of $10 \mathrm{~Pa}^{2}$ would tend to emphasize the measurements excessively and lead to more inconsistent state estimates. In contrast, for data sets in which the pressure had a peak magnitude of about 10-20 $\mathrm{Pa}$, the $10 \mathrm{~Pa}$ standard deviation level would not respond sufficiently to the measurements. Perhaps this could lead to an adaptive implementation of the extended Kalman filter, in which the measurement noise covariance magnitude depends on the magnitude of the measured pressure. However, for this investigation, a variance of $10 \mathrm{~Pa}^{2}$ was used consistently for the diagonal of the measurement covariance matrix.

\subsubsection{Streamlined Body Results}

In both experimental setups described in this chapter, the nearest vortices were able to be successfully tracked in position and strength using a combination of the forward model, the 
extended Kalman filter, and its tuned internal parameters. In looking into the details, it will be possible to further understand under what conditions vortices can be tracked.

The experiments which enclosed a linear array of pressure sensors in a streamlined tubular body required a 1.3 vortex forward model, which recreated the pressure at the sensors accurately by not modeling the presence of the body. In looking at the results of the inverse problem applied to this situation, the state of the nearest vortex is tracked surprisingly well under a number of conditions. In the presence of substantial unmodeled signal sources, the state estimate would robustly return to an accurate value after the unexplained pressure signal abated.
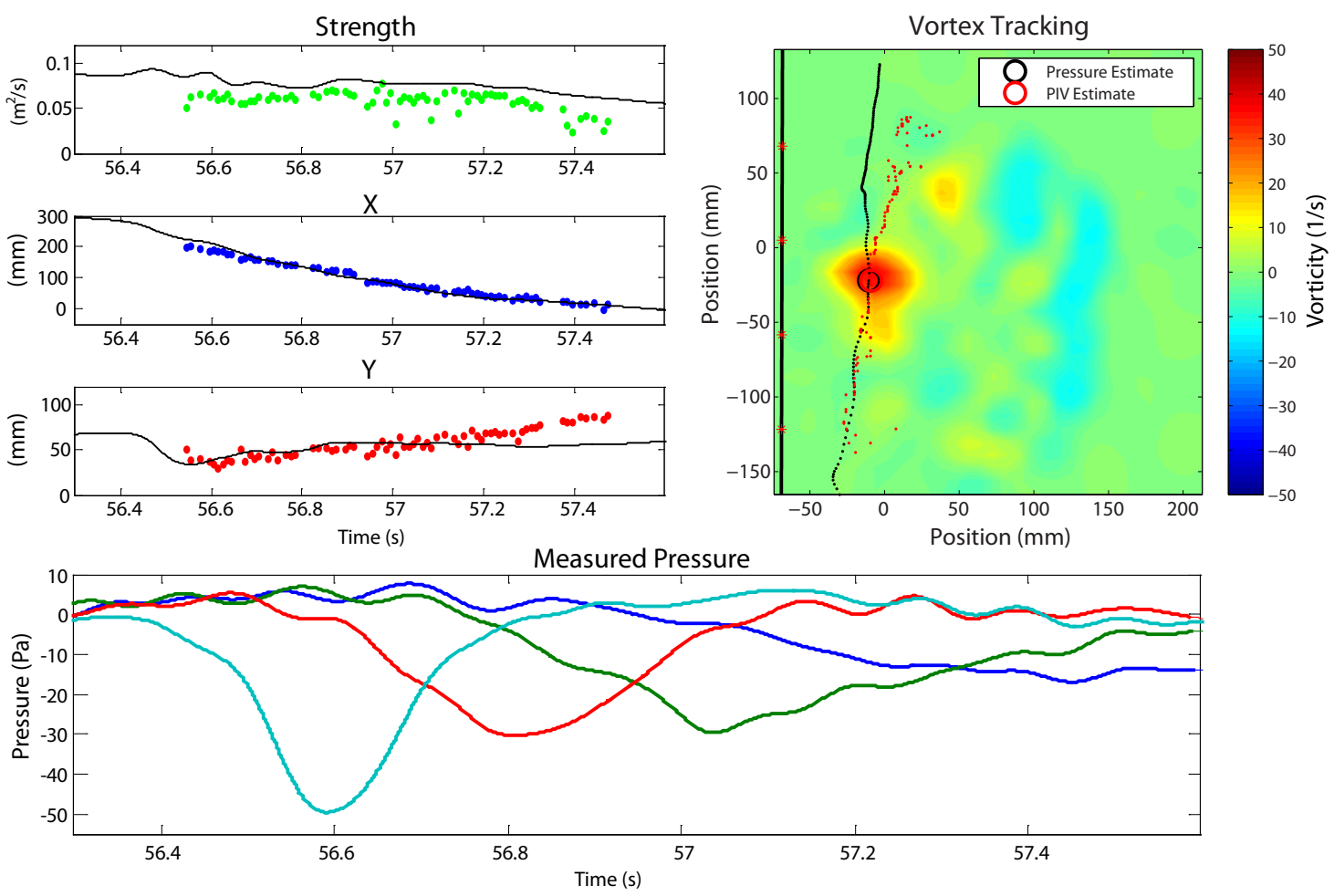

Figure 5-13: Results of a streamlined body vortex estimation trial. The estimated state variables (black line) are compared against the values from the PIV data in the upper left. In the upper right, the position traces of the pressure-estimated (black points) versus PIVestimated (red points) vortices are shown against the background of the vorticity distribution at an intermediate time. The edge of the sensor body and sensor locations are marked at the left edge of the image. At the bottom, the measured pressure is shown, filtered with a $20 \mathrm{~Hz}$ cutoff frequency. 
The first two examples of the state estimation for a streamlined body are examples of data sets without substantial aberrations in the pressure. Figure 5-13 shows the results of the vortex estimation from the linear pressure sensor array, compared against the estimate independently derived from the PIV data. The estimates of each state represent the mean of the conditional probability for each state variable conditioned on all the pressure measurements up to and including the time of the estimate. Focusing first on the upper left section of the figure, each state estimate based on pressure follows the behavior of the true state as derived from the PIV closely. The best of these is the $x$ position, denoting the location of the vortex along the length of the array. The estimate of the $x$ position matches the PIV data almost exactly. In contrast, the $y$ position estimate based on pressure tracks the true position of the vortex very well throughout the majority of the data set, but smoothly drifts apart at the tail end. The strength estimate appears to have a constant bias, overestimating the strength of the vortex in comparison to the PIV results, but does capture the slight decay in strength throughout the data set. Unfortunately the PIV that corresponds to this run decays in quality slightly at the tail end, making the the drift away from the sensors slightly suspect. This is visible in the righthand plot, where the PIV estimate of the location begins to lose cohesion. However, the main cause of the failure to track the small drift is likely within the parameters of the Kalman filter. It is possible that by that point, the data had been consistent with the model to the point that the influence of new measurements was reduced. Another visualization of the results for this example is shown in Figure 5-14, in which the location of the vortex estimates from the pressure and PIV are shown against the corresponding vorticity distribution at different points in time.

In comparing the state estimate results with the measured pressure, there is a clear adjustment in the estimate of the $y$ position that occurs at approximately 56.4 seconds. This corresponds to the time where the first sensor is beginning to differ significantly in comparison to the others. This behavior is expected, as before that point in time there is little information available that may not be attributed to noise in the measurements. However, it does also bring up an important point. The approach used here to track a vortex makes substantial use of the initialization transients of the Kalman filter. Since very little is assumed known about the location and size of the vortices, the initial period in which the estimate 
Streamlined Body Estimation Results
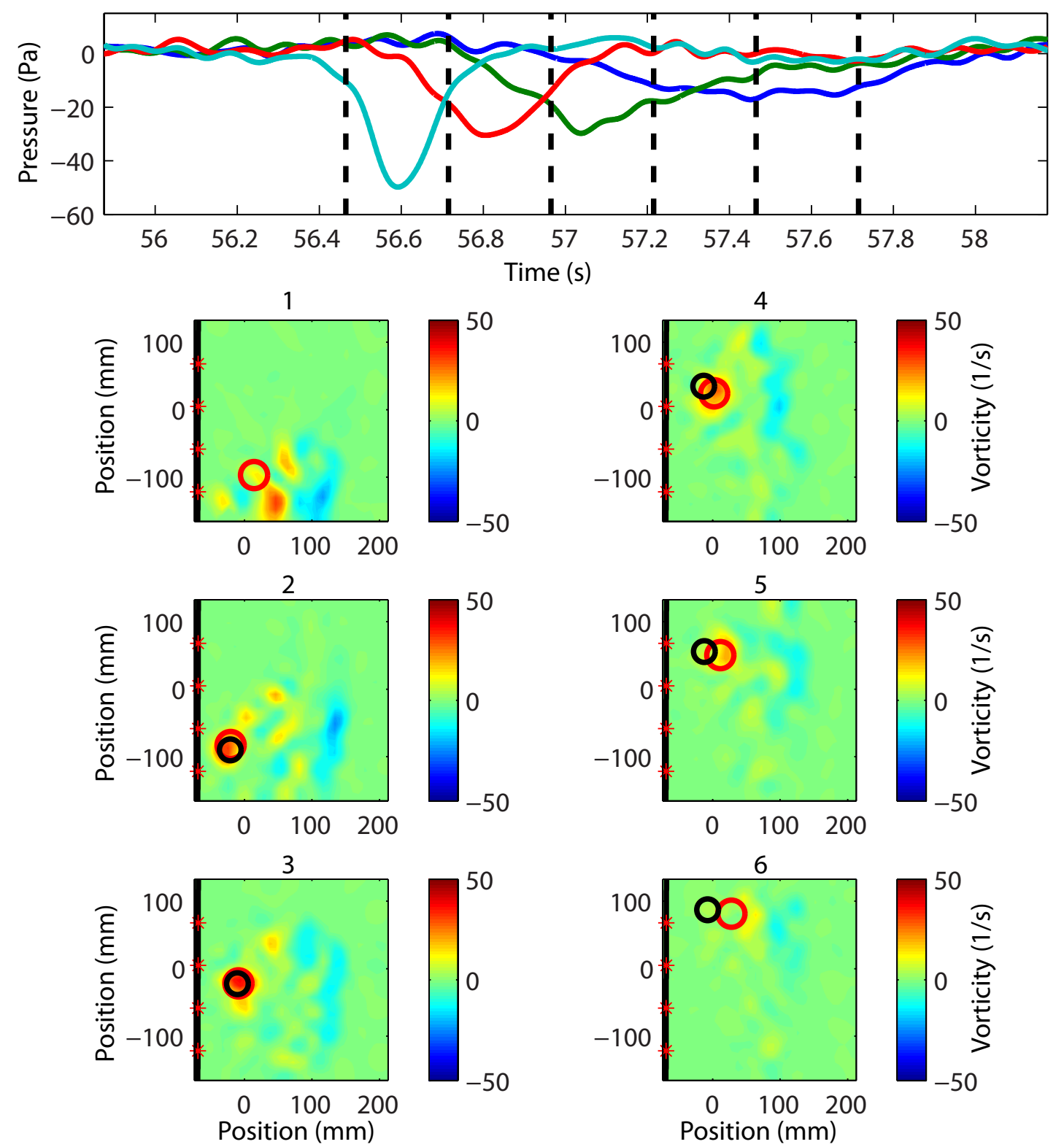

Figure 5-14: Results of a streamlined body vortex estimation trial. The results correspond to the same data as Figure 5-13. The position estimate based on the PIV data (red circle) is compared against that based on the pressure data only (black circle) at different stages in the time series. Each of the smaller subfigures, proceeding vertically from the top left, corresponds to the time marked by a dotted line in the top subfigure. 
is more sensitive to the measurements than the steady state behavior is very important in locating the vortices.

The second of the clean examples demonstrates a scenario that is slightly different from the first, in which the extended Kalman filter implementation does follow a signification change in the motion of the nearest vortex. These results are visible in Figure 5-15 and Figure 5-16. Although in general the vortex state is tracked very well in both the examples so far, there are some substantial differences. Unlike the first example, this second one tracks the vortex strength well, without overestimating it. Additionally, as mentioned, the Kalman filter results track a substantial change in direction as the vortex approaches the sensor array in the $y$ axis after initially maintaining roughly constant. However, in further contrast to the previous results, the $y$ position estimate based on the pressure exhibits small oscillations about the true value. It is unclear what causes this behavior. One difference between this example and the previous is that the strength of the vortex is larger. This could be responsible for the improved tracking of the vortex strength, but would be unlikely to cause the oscillations. The most likely remaining cause is that some small secondary unmodeled vortices were present near the sensors, which were not sufficient to corrupt the vortex tracking, but did alter it.

It is important to note that in the discussion of the two examples so far, only the state of the closest vortex is being tracked. The 1.3 vortex model being used at the base of the extended Kalman filter is a model that includes two vortices, with the second vortex limited to one free variable representing the distance between the two vortices. However, in implementation, it was observed that this separation variable is greatly inconsistent and strongly underestimated in comparison to the observed (via PIV) location of the second vortex. This is most likely due to the greatly reduced sensitivity to the position of the furthest vortex when there is a closer one in the flow field. This difference in sensitivity had been previously observed in the simulated tests of the extended Kalman filter, but it was trackable at that stage. With the inclusion of realistic noise and unmodeled fluid structures, it was determined that while this second vortex is necessary in order to obtain a good forward model, there can be no confidence in the inverse problem estimate of the second vortex. As a result, the vortex tracking is focused on the issue of accurately tracking 

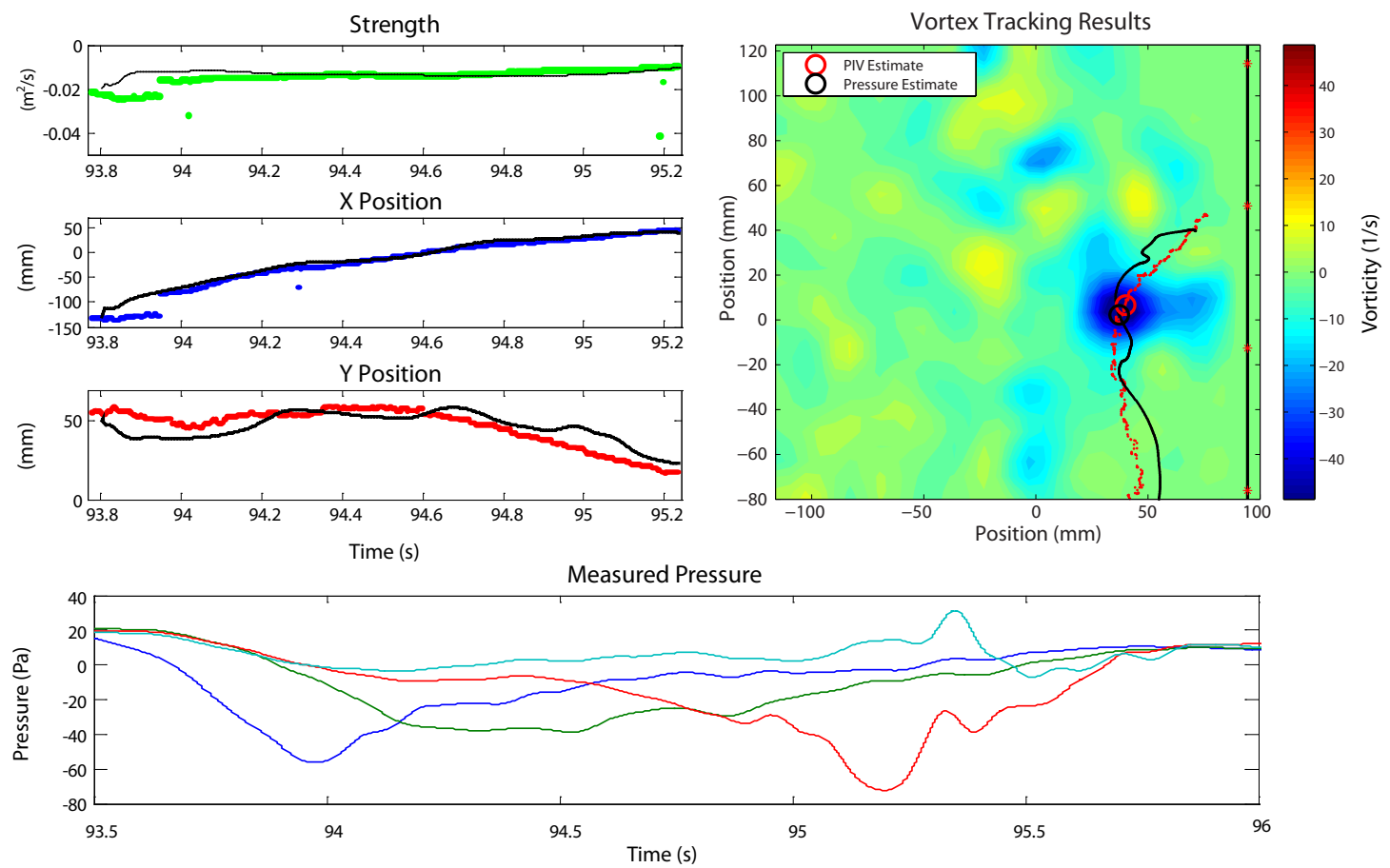

Figure 5-15: Results of a streamlined body vortex estimation trial. The estimated state variables (black line) are compared against the values from the PIV data in the upper left. In the upper right, the position traces of the pressure-estimated (black points) versus PIVestimated (red points) vortices are shown against the background of the vorticity distribution at an intermediate time. The edge of the sensor body and sensor locations are marked at the right edge of the image. At the bottom, the measured pressure is shown, filtered with a $20 \mathrm{~Hz}$ cutoff frequency. 

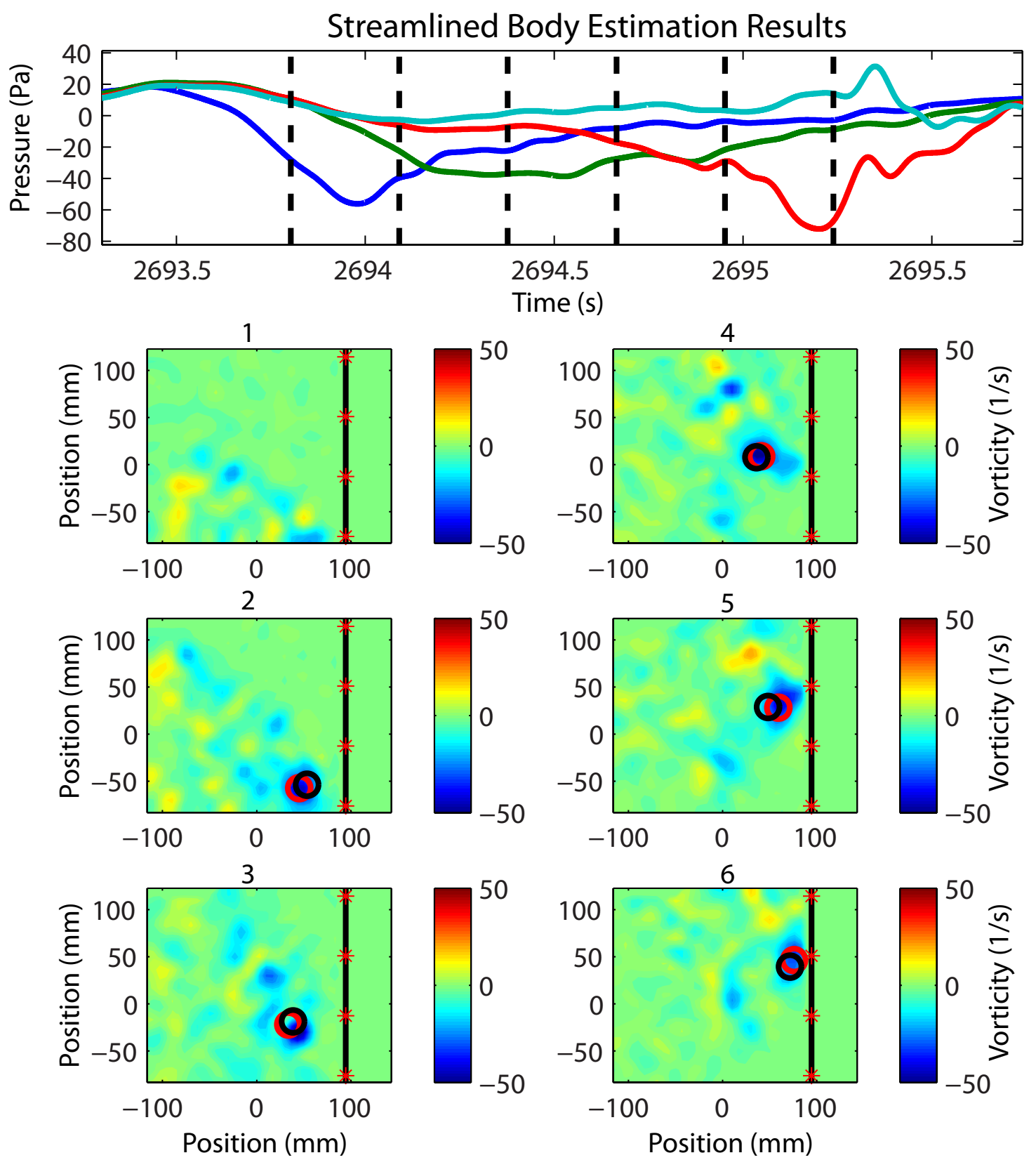

Figure 5-16: Results of a streamlined body vortex estimation trial. The results correspond to the same data as Figure 5-15. The position estimate based on the PIV data (red circle) is compared against that based on the pressure data only (black circle) at different stages in the time series. Each of the smaller subfigures, proceeding vertically from the top left, corresponds to the time marked by a dotted line in the top subfigure. 
the closest vortex only.

In considering other examples of the application of the extended Kalman filter vortex tracking approach, it is possible to observe what occurs when there is a faulty sensor in the array, or when a clearly anomalous transient signal is introduced. Given that the streamlined body enclosed only four sensors in the pressure sensor array, the importance of each sensor is increased. This is visible in the next two examples, Figures 5-17 to 5-20. In both of these figures, the first pressure sensors in the order passed by the vortex has an offset of approximately $10 \mathrm{~Pa}$. Recall that the Kalman filter implementation is using the difference in normalized pressure sensors, where the normalization occurs by removing the mean based on a time sample spanning several common mode oscillations away from the vortex stimulus. In the case of a normal sensor, this reference level normalization is constant throughout the experimental test. However, in the first sensor for each of these examples, it appears that the reference level changes during the passing of the vortex. This could possible be accounted for by a bubble in the line leading from the external port to the sensor inside the body that shifted when the pressure changed due to the passing vortex. Regardless of the cause, it is clear that there is a drift in the reference level of the sensor due to the way that the pressure difference against the adjacent sensor does not return to zero at both sides of the data set.

The result of this simple $10 \mathrm{~Pa}$ drift in the first sensor is dramatic. Until the pressure measured by the first sensor equalizes with the reference level, the vortex state estimate is corrupted. This is especially true of th $y$ position estimate, which is forced much closer to the erroneous sensor. Interestingly, the vortex state estimate recovers to an accurate state fairly quickly in both cases after the influence of the drifted vector is past. The speed of recovery may have something to do with the manner in which the strength and $x$ position components of the state are reasonable estimates of the true state. The amount of error in these state estimates is larger than that found in the examples with four working sensors, but is nowhere near the level of the $y$ position estimate. In fact, the $x$ position is tracked almost as well as in the examples with a full set of working sensors. Overall, it appears that an offset sensor generates minor disturbances in the $x$ and $k$ estimates, but causes the $y$ estimate to deviate wildly from reality. 

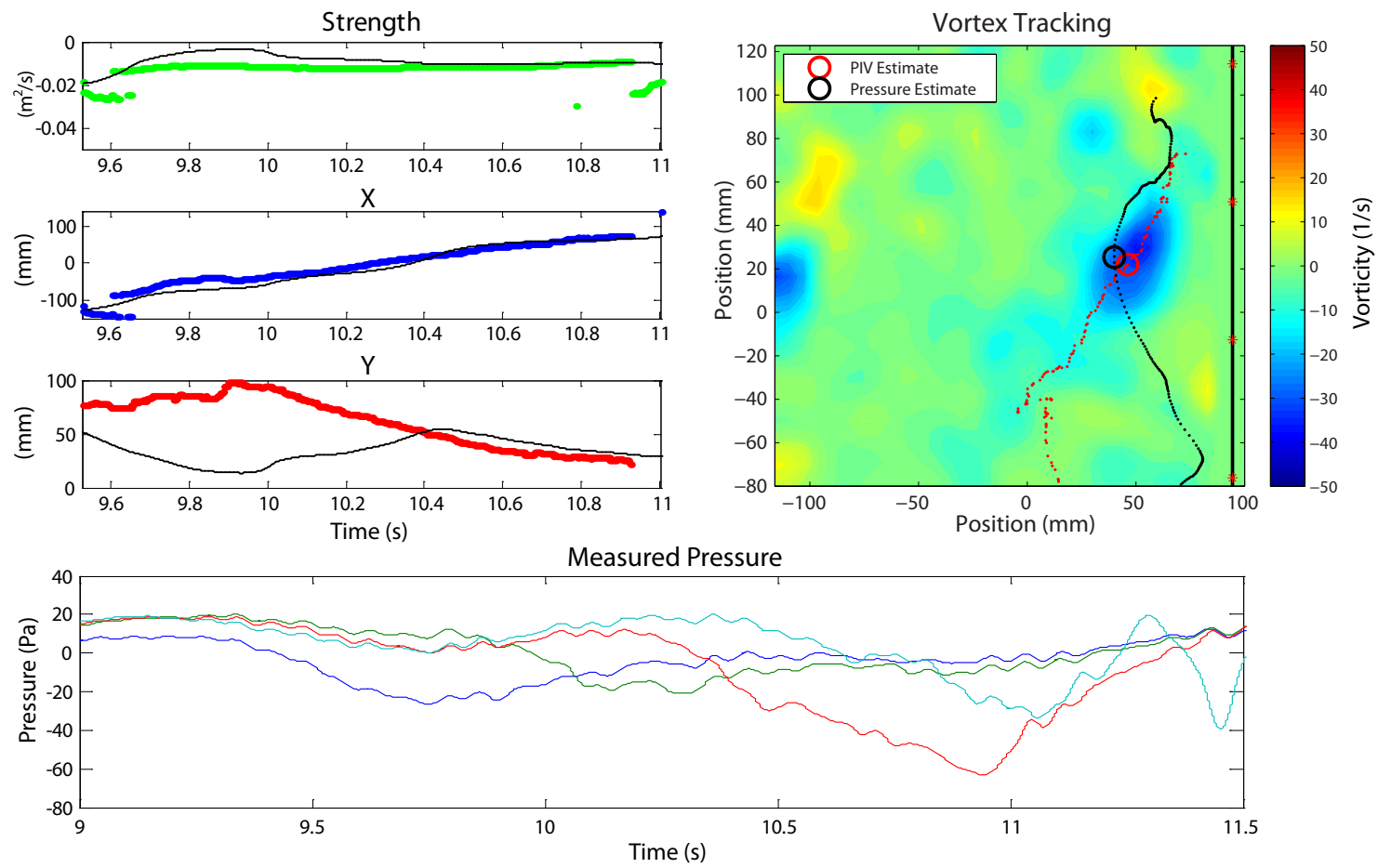

Figure 5-17: Results of a streamlined body vortex estimation trial. The estimated state variables (black line) are compared against the values from the PIV data in the upper left. In the upper right, the position traces of the pressure-estimated (black points) versus PIVestimated (red points) vortices are shown against the background of the vorticity distribution at an intermediate time. The edge of the sensor body and sensor locations are marked at the right edge of the image. At the bottom, the measured pressure is shown, filtered with a $20 \mathrm{~Hz}$ cutoff frequency. 

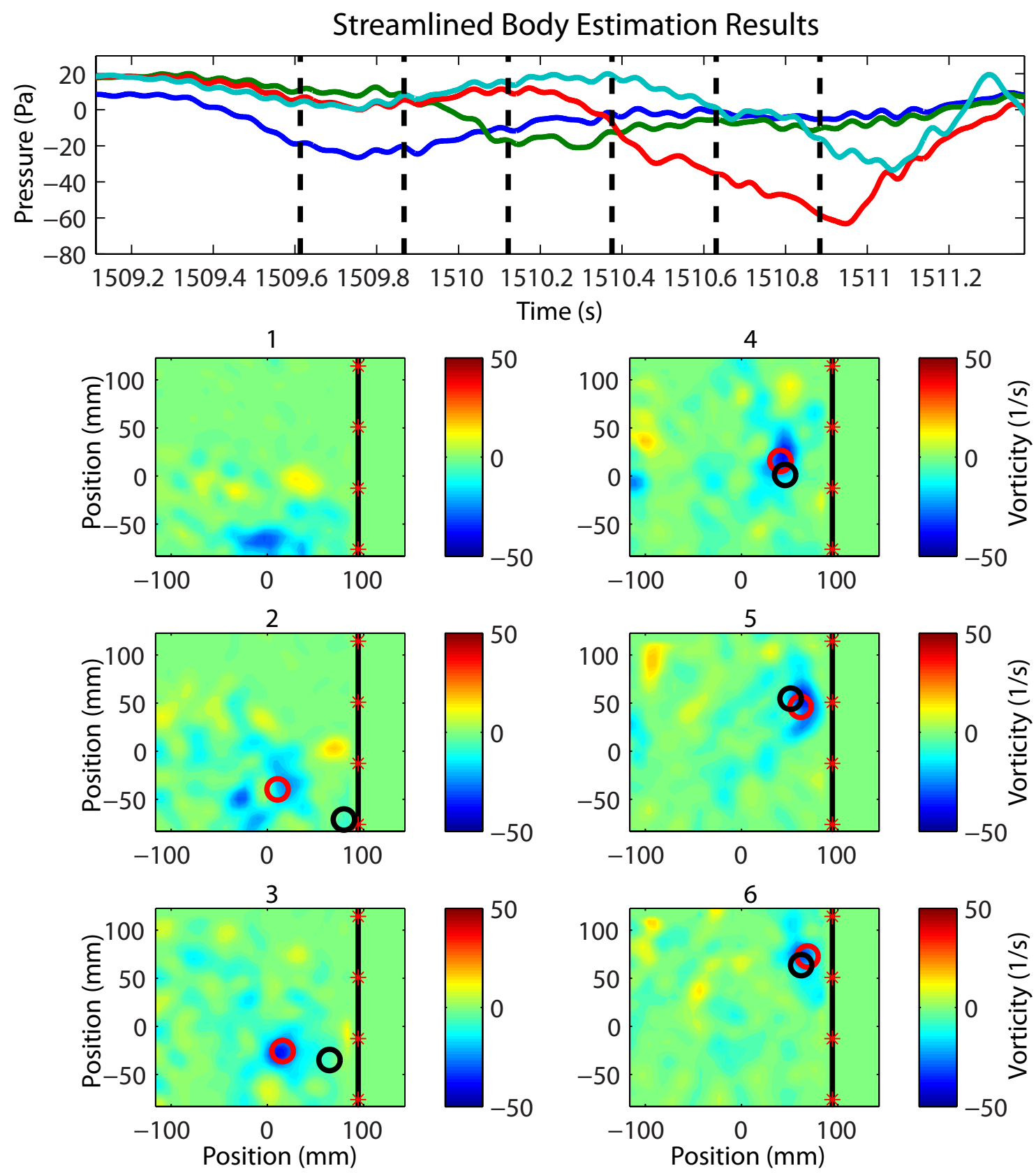

Figure 5-18: Results of a streamlined body vortex estimation trial. The results correspond to the same data as Figure 5-17. The position estimate based on the PIV data (red circle) is compared against that based on the pressure data only (black circle) at different stages in the time series. Each of the smaller subfigures, proceeding vertically from the top left, corresponds to the time marked by a dotted line in the top subfigure. 

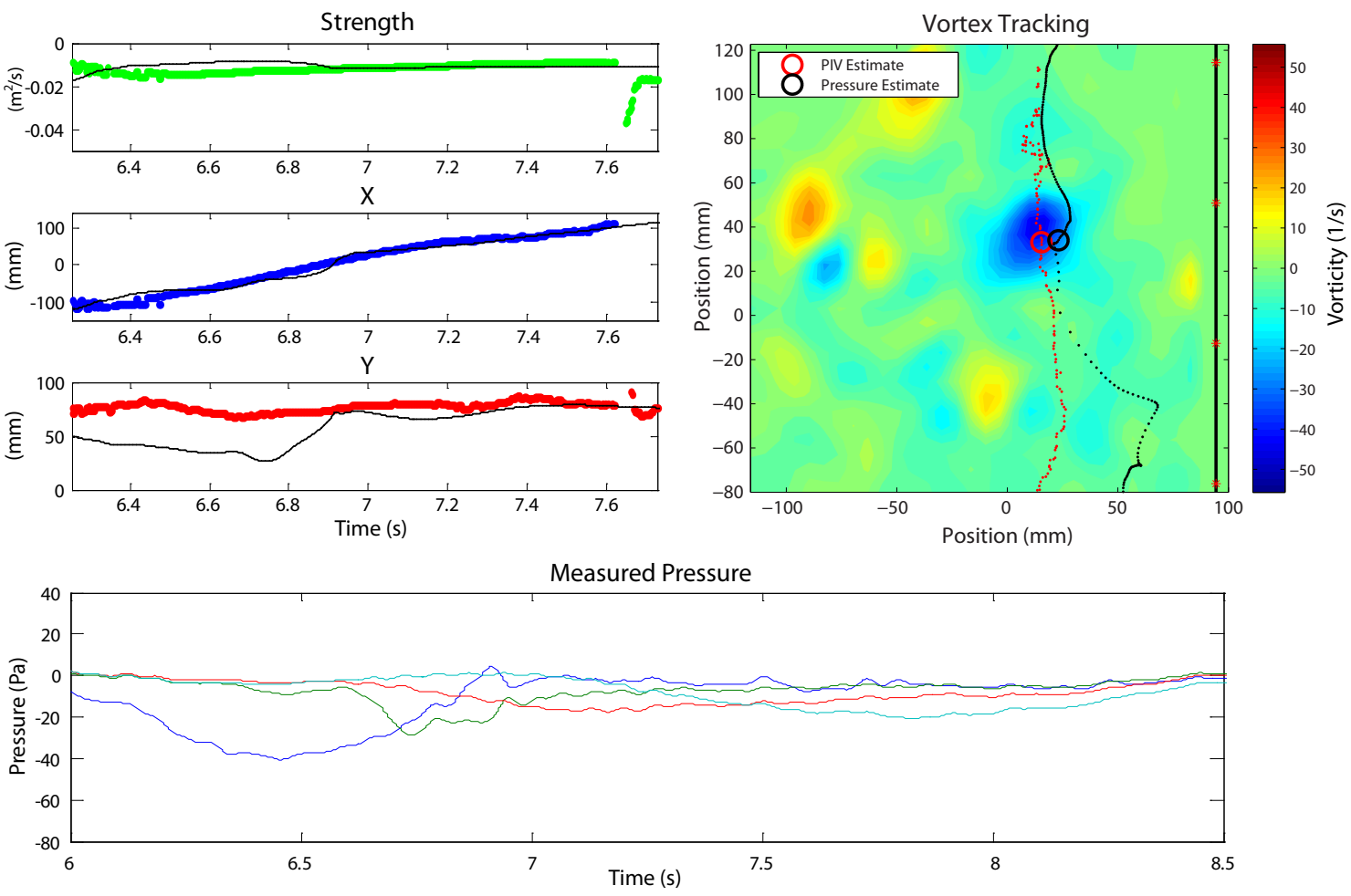

Figure 5-19: Results of a streamlined body vortex estimation trial. The estimated state variables (black line) are compared against the values from the PIV data in the upper left. In the upper right, the position traces of the pressure-estimated (black points) versus PIVestimated (red points) vortices are shown against the background of the vorticity distribution at an intermediate time. The edge of the sensor body and sensor locations are marked at the right edge of the image. At the bottom, the measured pressure is shown, filtered with a $20 \mathrm{~Hz}$ cutoff frequency. 

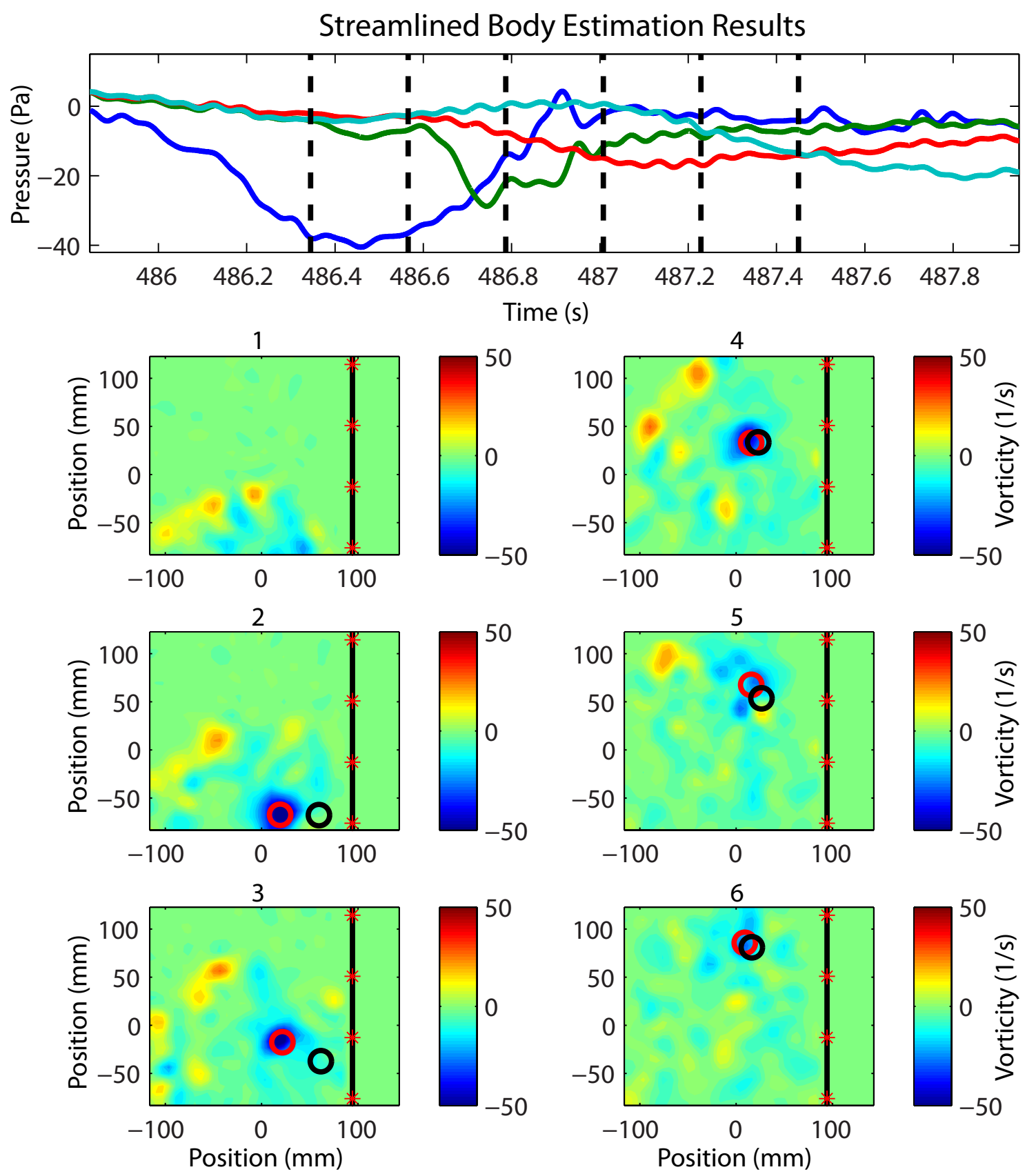

Figure 5-20: Results of a streamlined body vortex estimation trial. The results correspond to the same data as Figure 5-19. The position estimate based on the PIV data (red circle) is compared against that based on the pressure data only (black circle) at different stages in the time series. Each of the smaller subfigures, proceeding vertically from the top left, corresponds to the time marked by a dotted line in the top subfigure. 
Another example of vortex tracking from a streamlined body in the face of suboptimal operating conditions is displayed in Figures 5-21 and 5-22. In this experimental result, the measured pressure has an aberration at approximately 961.8 seconds, where the pressure in the third sensor suddenly increases for a brief period of time. The cause of this increase is unknown, the it appears from the PIV that the near vortex in this case was closer to the sensors than the others considered so far, so it's possible that secondary vortices may have formed at the interface with the sensory body. Regardless of the cause, the vortex tracking algorithm is unable to accurately track the nearest vortex while one of the sensors is measuring a large stimulus that is not due to the vortex in question. Again however, once the transient unexplained signal passes, the estimate based on pressure data appears to recover to track the PIV estimate once again (Note that the PIV estimate becomes sporadic afterwards due to the proximity of the solid body).

As demonstrated by a number of examples, a small linear sensor array of four pressure sensors is sufficient to track a nearby vortex from a small streamlined body, such as a fish or AUV. These results also validate the 1.3 vortex model, with the assumption that the streamlined body does not influence the flow for the purposes of predicting the pressure measurements. The level of detail available to a lateral-line-like sensor is as high as could be desired. With a full set of functioning sensors, the position of a vortex center was tracked with a mean error of a few millimeters, smaller than the viscous core of the vortices in question. These successful results also confirm that a fish would have sufficient information to extract this detailed information from a flow, focusing on the size and position of the nearest vortex to the lateral line. Whether a fish requires and uses this level of detailed inference over nearby vortices is unknown and a question to be addressed by experiments with the fish itself.

With only four sensors, it is evident that there is little redundancy in the measurements, and therefore even small discrepancies in the measurements of a single sensor can throw off the vortex tracking. However, it is also clear that the vortex tracking estimate can recover from a faulty sensor after the vortex passes beyond that sensor. In the case of a larger number of sensors, it would likely be possible to correctly estimate the state of a vortex while a single sensor provides faulty readings. 

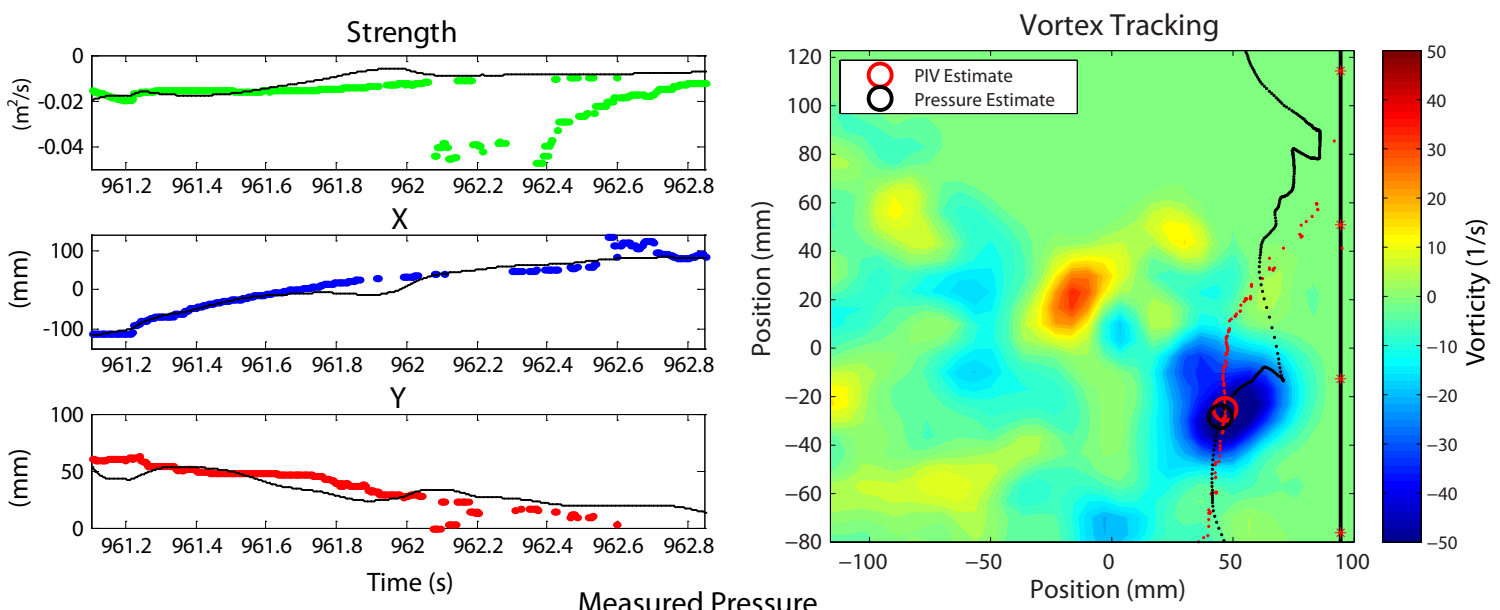

Measured Pressure

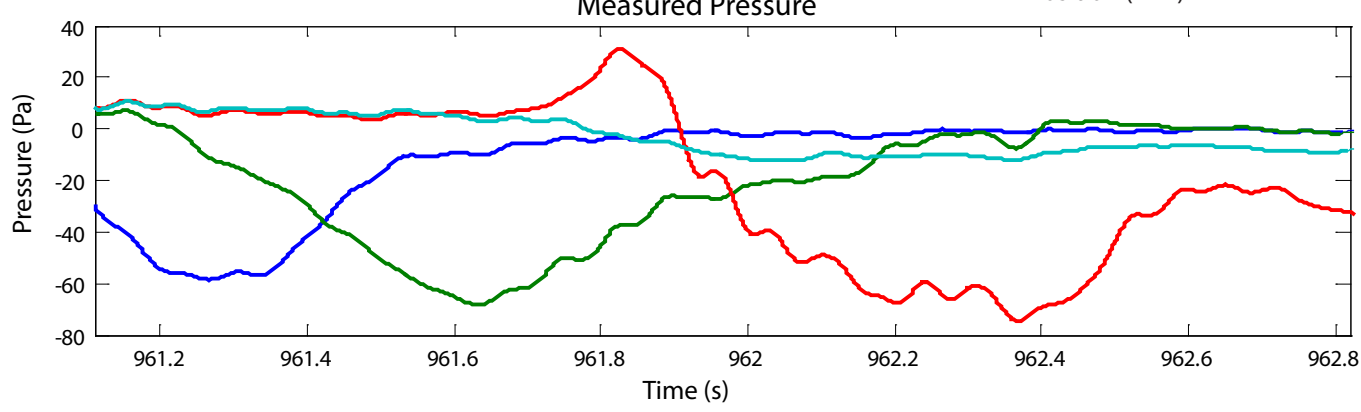

Figure 5-21: Results of a streamlined body vortex estimation trial. The estimated state variables (black line) are compared against the values from the PIV data in the upper left. In the upper right, the position traces of the pressure-estimated (black points) versus PIVestimated (red points) vortices are shown against the background of the vorticity distribution at an intermediate time. The edge of the sensor body and sensor locations are marked at the right edge of the image. At the bottom, the measured pressure is shown, filtered with a $20 \mathrm{~Hz}$ cutoff frequency. 
Streamlined Body Estimation Results
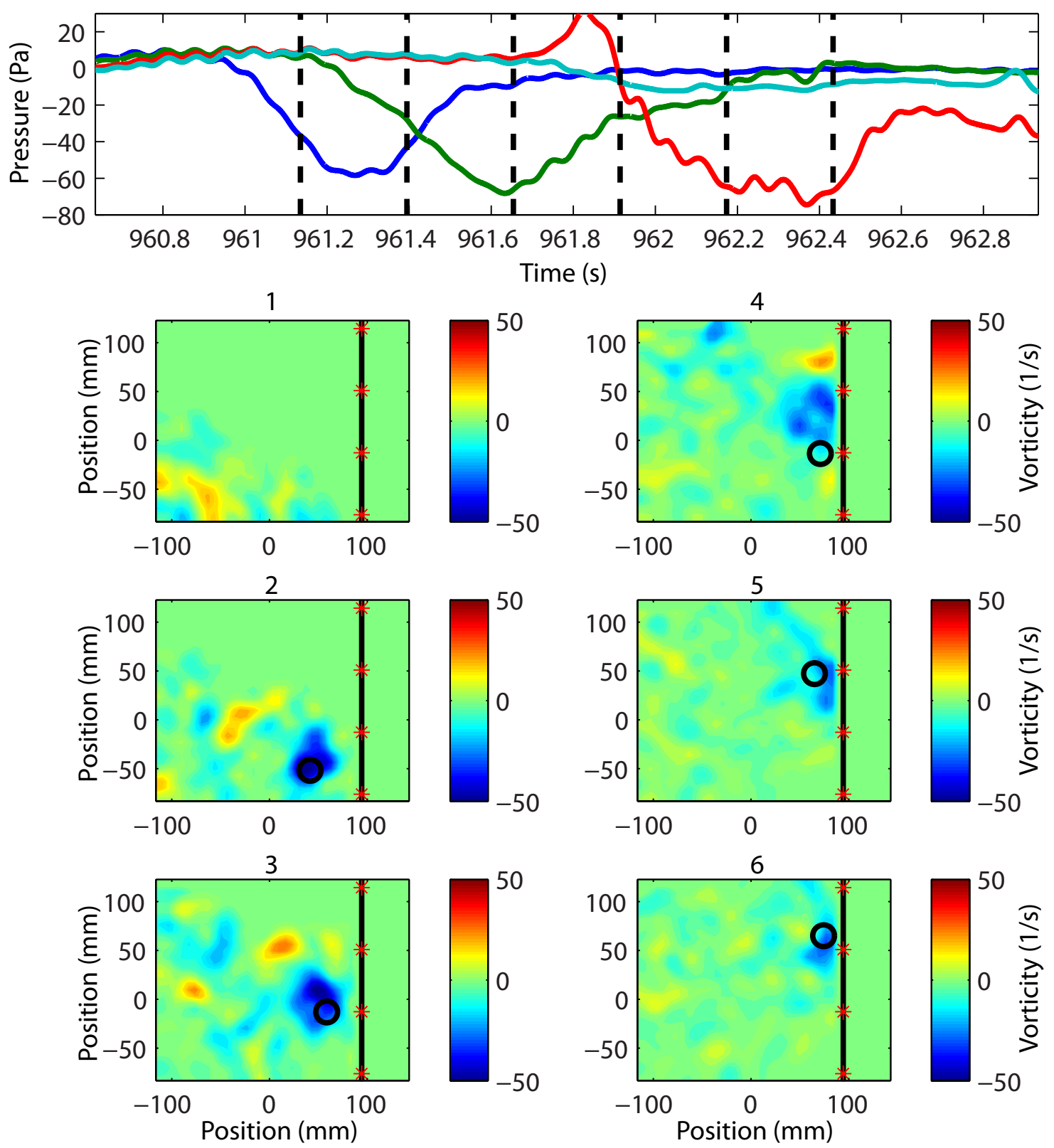

Figure 5-22: Results of a streamlined body vortex estimation trial. The results correspond to the same data as Figure 5-21. The position estimate based on the PIV data (red circle) is compared against that based on the pressure data only (black circle) at different stages in the time series. Each of the smaller subfigures, proceeding vertically from the top left, corresponds to the time marked by a dotted line in the top subfigure. 


\subsubsection{Flat Plate Results}

The results from estimating the state of a vortex from a sensor array embedded on a flat plate are similar to those for the streamlined body, despite the different model used in the extended Kalman filter. In addition to the model used, there are substantial differences in the number of sensors (Six active sensors versus four in the previous results) and in the trajectory of the tracked vortex.

The original pressure sensor array enclosed by the flat plate contained seven pressure sensors evenly distributed over a line. However, during the experiments the third sensor malfunctioned. The error in the measured pressure from this sensor consisted of persistent intermittent jumps of roughly equal magnitude. Unlike the case when the sensor drifted for the experiments with the streamlined body, in this case the entire sensor was simply discounted when considering the observed pressure. For the calculation of differences between adjacent sensors, one of those differences now corresponds to the gradient over twice the distance of the others. Since there are six remaining sensors, the sensor array is still sufficient for tracking a vortex as shall be seen shortly.
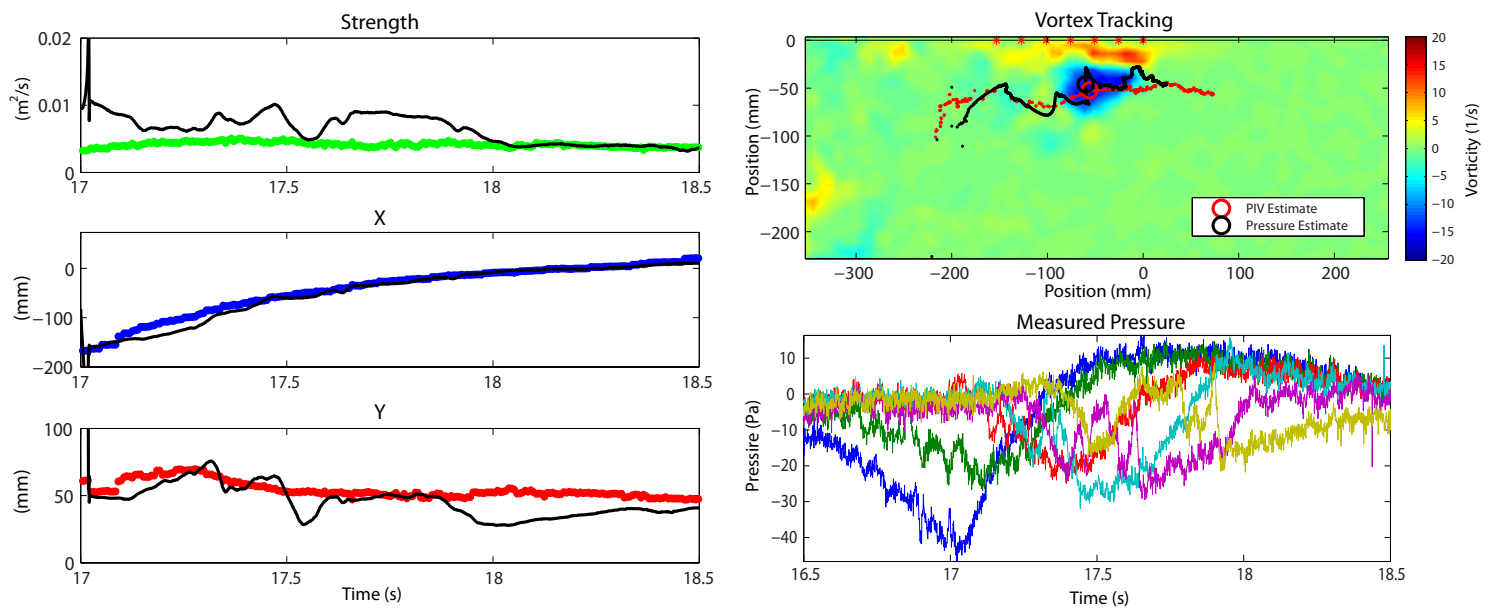

Figure 5-23: Results of a vortex estimation trial with the sensors on a flat plate. The estimated state variables (black line) are compared against the values from the PIV data at the left. In the upper right, the position traces of the pressure-estimated (black points) versus PIV-estimated (red points) vortices are shown against the background of the vorticity distribution at an intermediate time. The edge of the sensor body and sensor locations are marked at the top edge of the image. At the lower right, the unfiltered measured pressure is shown. 
A number of details due to the difference in experimental setup are worth discussing before examining the accuracy of the resulting vortex estimates. First of all, due to the path of the initial vortex pair, there is a large signal measured by the first sensor in all the data sets which does not contribute towards the tracking of the single nearby vortex. This first sensor is strongly influence by the presence of the second vortex which subsequently moves away from the sensors in the opposite direction. This is evident in the blue pressure trace in Figure 5-23. This scenario is somewhat artificial from the point of view of tracking externally generated vortices. The focus of these tests is to evaluate whether a lateralline-like array can identify and track a single vortex near a hull-like body, so the extended Kalman filter is initialized slightly late, removing some of the initial data in which the single vortex model is completely invalid. Besides the initial vortex pair, the pressure data obtained from the flat plate experiments tended to be of a smaller magnitude than those from the streamlined body. This is likely more related to the manner in which the vortices were generated, which required a larger distance and weaker torques.

Finally, another major difference between the streamlined body experiments and those with the flat plate is the level of interaction between the vortices and the sensing body. This difference is in part the entire reason for the two different sets of experiments. However, it appears that the level of interaction between the vortex and flat plate goes slightly beyond that captured in the reflected-vortex model. In both cases, the vortex centers appear to approximately $50 \mathrm{~mm}$ from the sensor array. However, with the streamlined body, there appeared to be no secondary vorticity generated by an interaction between the vortex and the boundary layer on the body. In contrast, with the flat plate body, a noticeable shear layer does develop on the plate surface (vorticity distribution in Figure 5-23), and eventually small counter-rotating vortices are shed into the flow (frames 4 and 6 in Figure 5-24).

A typical result for the flat-plate-embedded sensors is shown in Figure 5-23. As mentioned, the extended Kalman filter is initialized slightly after the first disturbances in pressure due to vortices are felt. However, this does not completely bypass the influence of the second physical vortex. At the $17 \mathrm{~s}$ mark, where the estimation begins, the first sensor is at its peak measurement. The vortex tracking result demonstrate that, despite the initial second vortex and the later secondary vortices, the estimate of the $x$ position along the length 
of the array quickly converges to the correct value and tracks the slowing progression of the vortex very accurately. The strength and $y$ position on the other hand roughly track the true state of the vortex, but with some significant variability. Both the strength estimate seems to generally overestimate the equivalent PIV estimate, with the two converging at the end of the run. The $y$ position estimate based on pressure more closely tracks the PIV estimate throughout the data set, with a few intermittent regions in which the $y$ position is underestimated. The resulting path (Figure 5-23, upper right) is somewhat erratic but still tracks the passing vortex. Particularly with respect to the sudden deviations in the $y$ estimate, it appears that the erraticness of the estimate based on pressure is tied to the generation of secondary vortices. As is more clearly visible in Figure 5-24, two vortices clearly develop and shed off of the flat plate surface. These vortices are visible as they are being shed in frames 4 and 6 of Figure 5-24. These frames roughly correspond to the time just after the significant transitions in the $y$ position estimate occur. This implies that the effect of these smaller vortices as they form is sufficient to alter the pressure significantly, which is also not surprising given the close proximity of the vortices to the sensors.

Similar results are observed in a second data set, in which a slightly different behavior occurs with the secondary vortices. These results are shown in Figures 5-25 and 5-26. Note that in this data set, the third sensor (red pressure data in Figure 5-25) was not malfunctioning for the majority of the run and therefore was included in the problem inversion. The estimate of the vortex state again tracks the $x$ position well, while overestimating the strength and underestimating the $y$ position. In this case however, the underestimation of the $y$ position is more persistent than in the previous, which was largely on target with a few deviations. Based on the conclusions from the previous data set, the expectation is that this would be related to a difference in the secondary vortices being shed. This is born out through the PIV data, depicted in Figure 5-26. Unlike the previous data set, only a single counter-rotating vortex is shed from the boundary layer. This vortex is significantly stronger than the ones shed in the earlier run, and appears to persist much longer. It is possible that there is simply little time between the initial effect of the second physical vortex and the end effect of the shed secondary vortex for the the $y$ estimate to settle to the correct value. 
Flat Plate Body Estimation Results
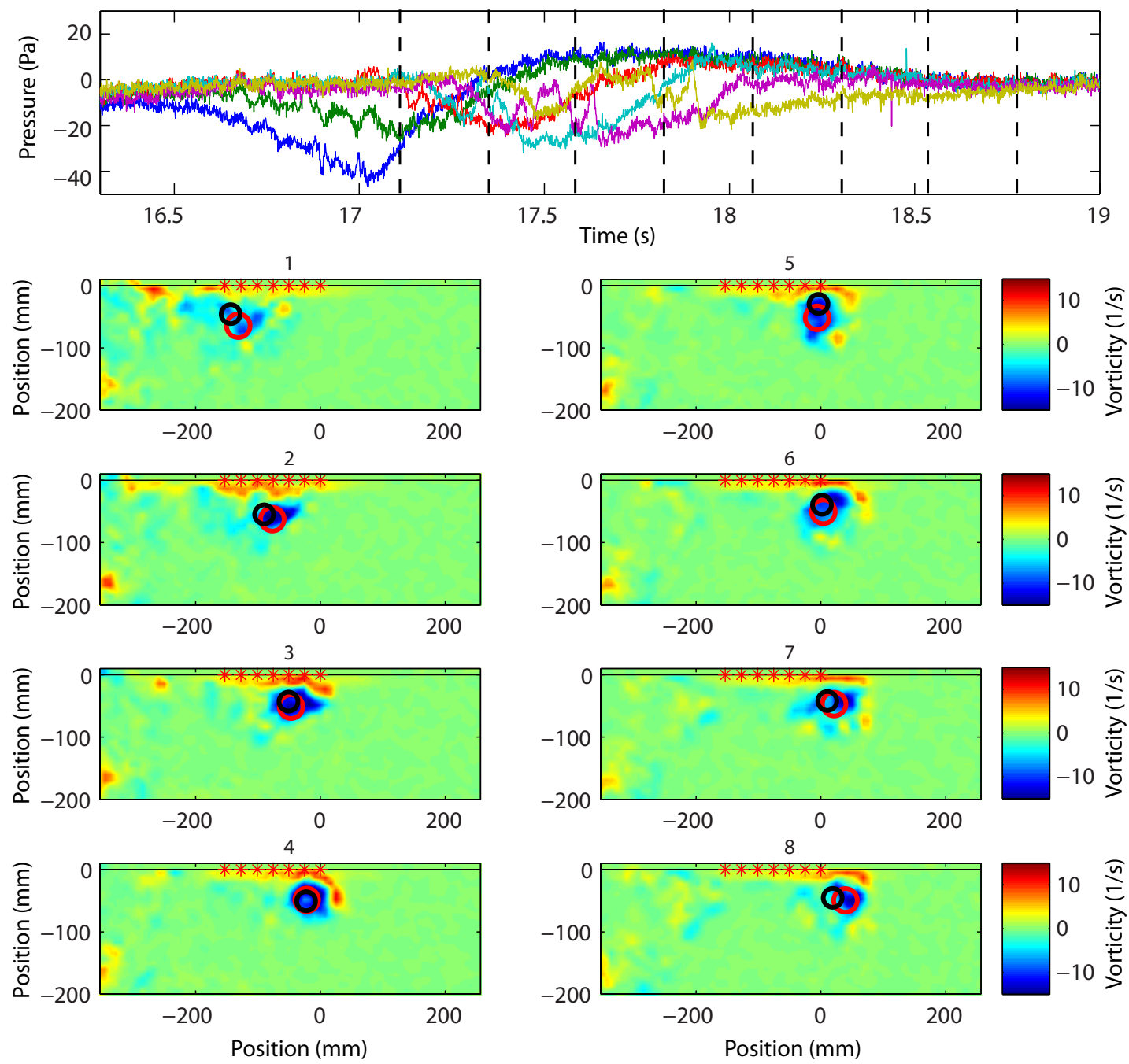

Figure 5-24: Results of a vortex estimation trial with the sensors on a flat plate. The results correspond to the same data as Figure 5-23. The position estimate based on the PIV data (red circle) is compared against that based on the pressure data only (black circle) at different stages in the time series. Each of the smaller subfigures, proceeding vertically from the top left, corresponds to the time marked by a dotted line in the top subfigure. 

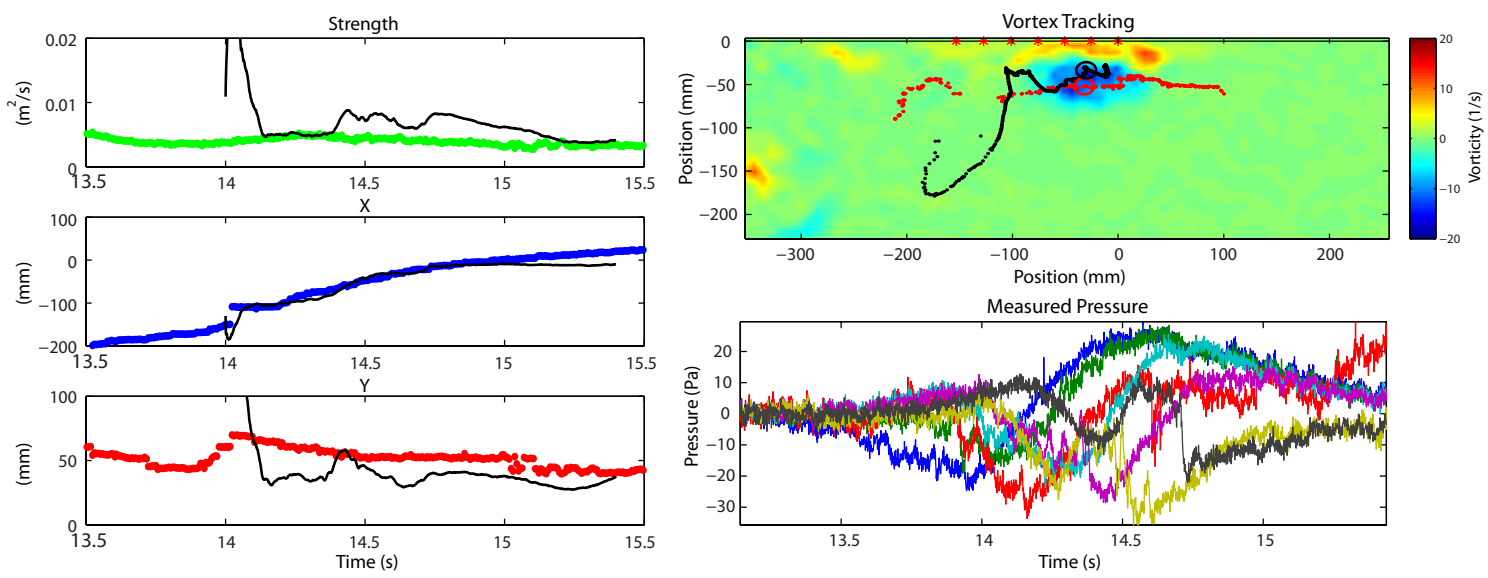

Figure 5-25: Results of a vortex estimation trial with the sensors on a flat plate. The estimated state variables (black line) are compared against the values from the PIV data at the left. In the upper right, the position traces of the pressure-estimated (black points) versus PIV-estimated (red points) vortices are shown against the background of the vorticity distribution at an intermediate time. The edge of the sensor body and sensor locations are marked at the top edge of the image. At the lower right, the unfiltered measured pressure is shown.

These results in tracking a vortex based on a lateral-line-like sensor array embedded in a flat plate indicate a surprising resilience in the estimation method to extra vortices in the flow. In particular, as secondary vortices are formed and shed into the flow from the interaction between the primary vortex and the flat plate, the $y$ estimate of the primary vortex state is altered by only approximately $1 \mathrm{~cm}$, close to the apparent radius of the vortex core. Although the approach developed here is best suited for externally generated vortices that do not interact heavily with the sensing body, it is reassuring in terms of any practical implementation that the violation of the assumptions leads to a modest bias in the vortex parameter estimation.

The proximity of the second physical vortex in the initially generated vortex pair made it difficult to estimate the state of the vortex of interest during the initial sections of the data sets. In future iterations these experiments in tracking externally generated vortices near a hull could be modified to put more distance between the sensors and the point at which the initial vortex pair separates. Based on the results observed in experiments described in this section, this would very likely lead to much better vortex tracking accuracy, though it 
Flat Plate Body Estimation Results
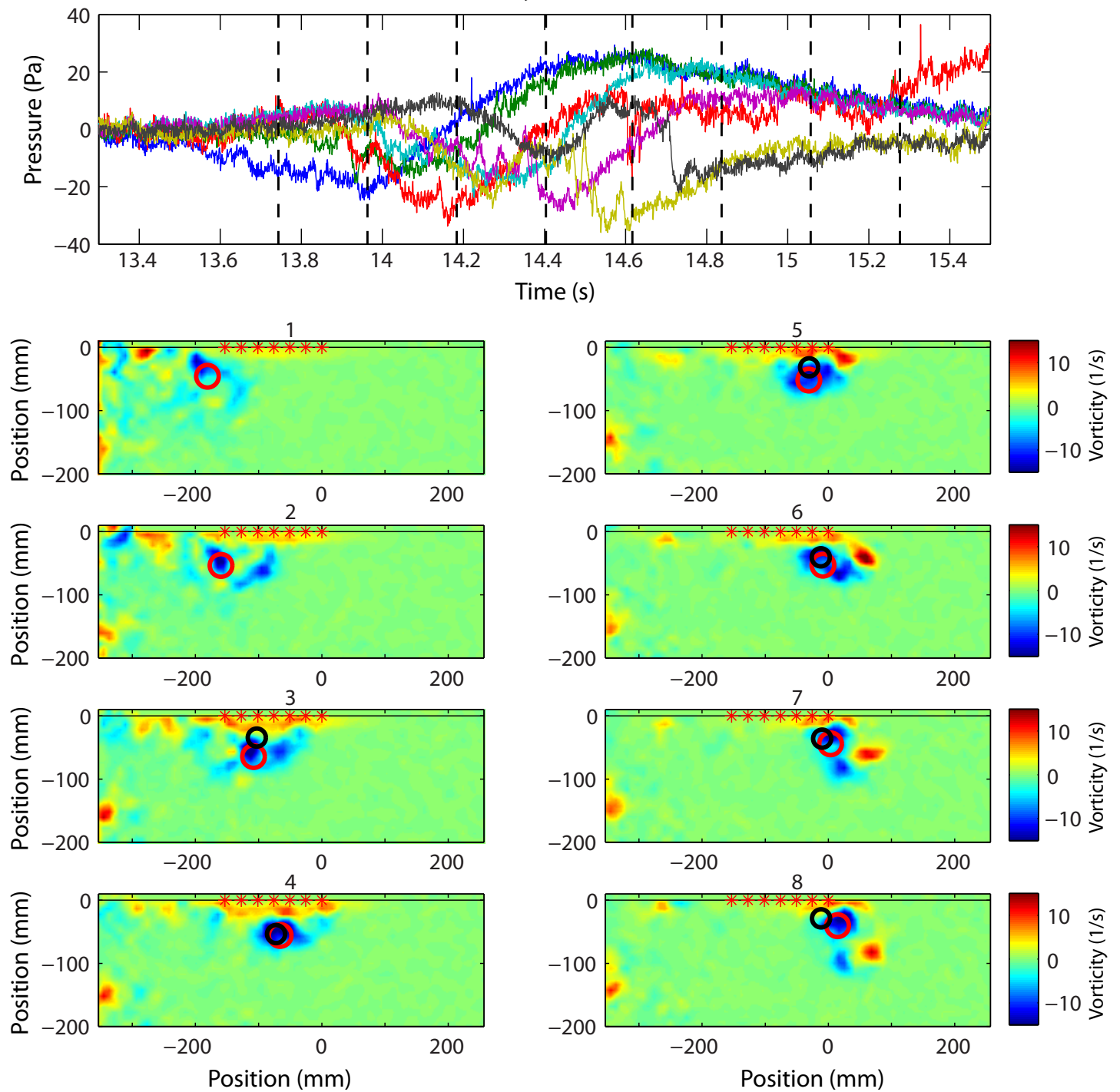

Figure 5-26: Results of a vortex estimation trial with the sensors on a flat plate. The results correspond to the same data as Figure 5-25. The position estimate based on the PIV data (red circle) is compared against that based on the pressure data only (black circle) at different stages in the time series. Each of the smaller subfigures, proceeding vertically from the top left, corresponds to the time marked by a dotted line in the top subfigure. 
would still not remove the effect of the secondary vortices.

\subsection{Vortex Tracking Dependence on Array Parameters}

Based on the experimental estimation results, it has been demonstrated that it is possible to track a vortex in an experimental setting under different conditions roughly corresponding to that of a fish and a ship hull. However, it is still unclear where the limits are to the vortex tracking approach described in this chapter. In particular, it is virtually certain that the ability to track a vortex using a linear array of pressure sensors will depend on the parameters of that sensor array: its overall length and sensor spacing. Considering the extreme case, a sensor array composed of a single sensor would clearly perform differently than the several-sensor arrays used in the experiments. It is critical to establish and understand the connection between the sensor array parameters and the ability to sense vortices in order to design appropriate and efficient lateral-line like sensor arrays using MEMS or other technologies, as is being done by a number of groups [35, 88].

In order to examine the relationship between the estimation of the vortex state and the sensor array parameters, the instantaneous estimate of the vortex state will be used. We will consider the best estimate of the vortex state given a single instantaneous set of measurements and no knowledge of the measurements or vortex state before and after. Considering the instantaneous inverse problem will make it possible to examine and identify the mechanisms which are responsible for affecting the performance of the vortex estimation. These mechanisms will then be extrapolated to estimation on the entire time series and compared to behaviors observed in the Kalman filter estimation. The procedure for this will first involve examining the effect of vortex location based on a single sensor and a representative small linear array in order to derive intuition on what motivates the spatial localization of a vortex. Then the effect of sensor array length and density will be examined for a vortex centered along the length of the array.

The instantaneous estimate of a vortex state is based on the minimization of a least squares cost function, in which $J(x, y, k) \propto \sum_{i}\left(P_{i}(x, y, k)-P_{i}^{\text {true }}\right)^{2}$. In this equation, the cost $\mathrm{J}$ represents a penalty based on the difference between the actual measured pressure 
and the simulated pressure of a vortex at position $(x, y)$ with strength $k$. The summation variable $i$ refers to a sum over the number of sensors used in the measurement. The least squares cost function is an appropriate choice for this analysis since it is at the heart of the Kalman filter, as well as being an intuitive cost function. Minimizing the cost function $\mathbf{J}$ corresponds to finding the vortex state that best generates the measured data.

\subsubsection{Reflected Vortex Model}

The two models used in the experimental vortex tracking will be considered separately. The first of these is the reflected vortex model, in which a single physical vortex is modeled in potential flow, with an imaginary vortex reflected over the sensor array line. Recall that the complex potential of the reflected vortex model is described by:

$$
\Phi(z)=-i k \log \left(z-z_{o}\right)+i k \log \left(z-\overline{z_{o}}\right)
$$

where $z_{o}=x_{o}+i y_{o}$ represents the location of the physical vortex. The pressure associated with this model is derived using the unsteady Bernoulli equation in the same manner as discussed earlier.

With this model, it is possible to specify the cost function in more detail. The least squares cost function for this particular model can be written as follows:

$$
J\left(x_{o}, y_{o}, k_{o}\right)=\frac{1}{\left(P_{o}^{\text {true }}\right)^{2}} \sum_{i=1}^{N}\left(P_{i}\left(x_{o}, y_{o}, k_{o}\right)-P_{i}^{\text {true }}\right)^{2}
$$

Where $P_{i}^{\text {true }}$ is the measured pressure at sensor $i$ obtained by the model based on the "correct" state of the vortex. If the test vortex state, denoted by the triplet $\left(x_{o}, y_{o}, k_{o}\right)$ is equal to the true state, it is clear that the cost $J$ will be zero and that this is the minimum value possible for the cost function. The constant $P_{o}^{\text {true }}$ in the cost function is a normalization constant that refers to the measurement with maximum magnitude based on the true vortex state. For a reflected vortex centered in the middle of an array, this will always correspond to the sensor location closest to the vortex.

Recall that the pressure at a point on the "wall" or plane of reflection is given by the 
following formula derived from the unsteady Bernoulli equation:

$$
\begin{aligned}
P(z)-P_{\infty} & =-\frac{1}{2} \rho\left(\frac{\partial \Phi(z)}{\partial z}\right)^{2}-\rho \frac{\partial \Phi(z)}{\partial x_{o}} \frac{\partial x_{o}}{\partial t} \\
P(s)-P_{\infty} & =\rho k^{2} \frac{\left(s-x_{o}\right)^{2}-y_{o}^{2}}{\left(\left(s-x_{o}\right)^{2}+y_{o}^{2}\right)^{2}}
\end{aligned}
$$

Note that, as before, several shortcuts have been used implicitly due to the symmetry of the problem and the fact that the points of measurement are restricted to the real axis. For example, the complex potential is being used directly in the time derivative since the real axis forms a streamline. The variable $s$, a real scalar denoting the position of the measurement point on the real axis, is substituted in place of the complex $z$.

As evident from the end result, equation (5.12), the pressure measured at any point has a simple squared relationship with the strength of the vortex. This implies that the only effect of the vortex strength is on the magnitude of the signal, not its spatial distribution. As the main question being addressed is how the spatial relationships between the sensor array and the vortex position affect the ability to track the vortex state, the effect of the vortex strength is of secondary importance. Of course, if a vortex is too weak in comparison to the noise floor of the sensors, any attempts to estimate the vortex state will fail as there is effectively no information in the measurements. For this section, it will be assumed that the true vortex strength is sufficiently large compared to the noise amplitude.

When considering the cost of estimating a vortex to be at a particular location, the estimate of the vortex strength is unspecified and will therefore be chosen as the strength that minimizes the cost function at that point. This approach is intuitive in that two potential locations for a vortex will be compared by the most accurate pressure measurements that can be generated from those locations. It is also possible to consider the problem when the vortex strength is assumed known. This would correspond to having good a priori knowledge of the vortex strength due to either accurate earlier tracking or knowledge of the vortex formation mechanisms. In this case, the results look very different, but there is still ambiguity in the spatial position under certain conditions.

The optimization of the vortex strength for a given location follows directly from equa- 
tions (5.10) and (5.12). From equation (5.12), the pressure at a point is proportional to the strength squared: $P_{i}^{\text {true }}=k^{2} a_{i}$, where $a_{i}$ is the constant that depends on the location of the measurement and of course the location of the vortex. From this, the equation for the cost function can be rewritten and the root of its derivative with respect to $k$ found.

$$
\begin{aligned}
J(k) & =\frac{1}{\left(P_{o}^{\text {true }}\right)^{2}} \sum_{i=1}^{N}\left(k^{2} a_{i}-P_{i}^{\text {true }}\right)^{2} \\
\frac{d J(k)}{d k} & =\frac{2}{\left(P_{o}^{\text {true }}\right)^{2}} \sum_{i=1}^{N}\left(k^{2} a_{i}-P_{i}^{\text {true }}\right) 2 k a_{i} \\
0 & =\sum_{i=1}^{N} k^{2} a_{i}^{2}-a_{i} P_{i}^{\text {true }} \\
& =k^{2} \sum_{i=1}^{N} a_{i}^{2}-\sum_{i=1}^{N} a_{i} P_{i}^{\text {true }}
\end{aligned}
$$

The solution to the remaining quadratic equation gives the optimal vortex strength. In the instantaneous case, there is no information on the sign of the vortex, so by convention the positive strength will be used. In the experimental case of course, the motion of the vortex over time reveals the direction of rotation. At times, the quadratic equation (5.16) does not have real valued solutions. This corresponds to an impossible match between the measured pressure and the location of the test vortex. In these cases, the optimal strength is zero.

Instantaneous Least Squares Inversion Based on a Single Sensor
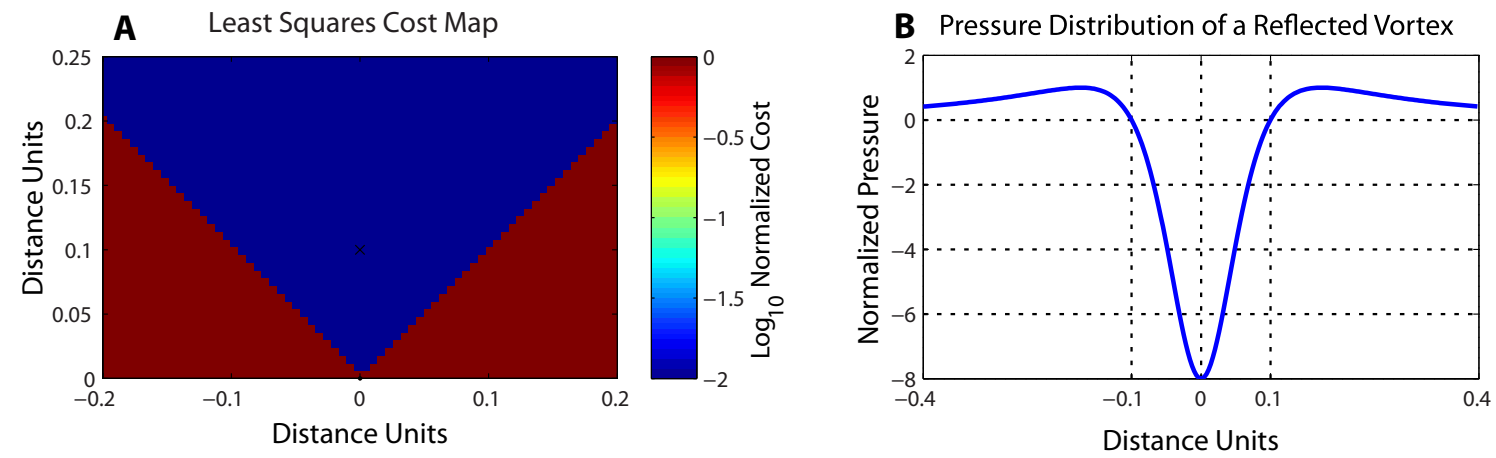

Figure 5-27: Instantaneous inversion based on a single pressure sensor for the reflected vortex model. Part A shows the spatial map of the cost function generated with one sensor. The small $\mathrm{x}$ at $(0,0.1)$ denotes the true location of the vortex. Part $\mathrm{B}$ depicts the pressure distribution on the wall at the $y=0$ axis. 
First consider the estimation of a vortex state for a vortex near a wall using only one sensor and a single measurement. Clearly one would expect a number of positions to be equally viable, since the strength of the vortex can be adjusted for a candidate position in order to recreate the measured pressure at the solitary point. For this particular model however, it turns out that not all points in the half plane (the fluid side of the plane bisected by the linear array) are valid. This is displayed in Figure 5-27, which demonstrates in subfigure A that only a vortex within a cone emanating at a 45 degree angle from the measurement point can generate the pressure measurement similar to that of a vortex directly in front of the sensor (marked by an small cross in the figure). The reason for this is clear from the distribution of pressure on the sensor line, shown at the right in Figure 527B. In the case of the reflected vortex model, the lines of zero pressure (compared to the far field stationary flow, as always) pass through the vortex location and form 45 degree angles with the reflection plane. This can also be observed directly from the numerator in equation (5.12). In the particular case shown in Figure 5-27, the vortex is 0.1 units away from the wall and the zero crossing points of the pressure on the wall are at 0.1 and -0.1 units. Within this range, the pressure is below the far-field pressure, but outside this range the pressure is above the far-field pressure. This spatial distribution is fixed relative to the center of the vortex, regardless of the location of the vortex or its strength. As a result, the sign of the pressure measured at a single point limits the possible range of the vortex that generated it. In the case of a single vortex, this restriction evidently does not aid the estimation much, as there is still a large region of ambiguity. However, the same principle plays a large role when there are multiple sensors.

A map of the cost function, as seen in Figure 5-27A and in the following figures, visually presents the spatial components of estimating the vortex position in an intuitive manner. In general, the cost function ranges from zero, at the exact location of the true vortex, to an upper limit that is typically $J_{\max }=\frac{1}{\left(P_{o}^{\text {true }}\right)^{2}} \sum_{i=1}^{N}\left(P_{i}^{\text {true }}\right)^{2}$, which corresponds to locations that cannot generate the same sign of pressure measurements as the true vortex. Although these maps of the cost function depict the difference between noiseless, simulated measurements, it is possible to relate the results to a more realistic measurement with random noise. Consider the cost when the noiseless measurement of the true vortex state is compared to 
a noisy measurement of the true vortex state: $J=\frac{1}{\left(P_{o}^{\text {true }}\right)^{2}} \sum_{i=1}^{N}\left(P_{i}^{\text {true }}-\left(P_{i}^{\text {true }}+\eta_{i}\right)\right)^{2}$ where $\eta_{i}$ is the noise variable for each sensor. This is the situation that would occur if the correct vortex state was hypothesized, but only noisy measurements were available. The result is $J=\frac{1}{\left(P_{o}^{\text {true }}\right)^{2}} \sum_{i=1}^{N}\left(\eta_{i}\right)^{2}$. If $\eta_{i}$ are normally distributed with variance $\sigma^{2}$, then $J$ becomes a Chi-Squared random variable $J=\frac{\sigma^{2}}{\left(P_{o}^{\text {true }}\right)^{2}} \chi^{2}$. This expression relates the cost function $J$ to the maximum pressure and measurement noise variance. For example, assuming that the noise variance is ten percent of the maximum pressure magnitude, which is a typical moderate noise scenario, it is possible to calculate a value for $J$ such that with 95\% confidence, the true state will be contained in the region with lower cost. This means that in general the regions of low cost in a spatial map of the cost function can be interpreted as being more easily confused through noise, and the relevant level sets of the cost map depend on the signal-to-noise ratio.

To examine the multiple sensor case with the reflected vortex model, first consider the effect of the position of the vortex with respect to a modest linear sensor array of five sensors. The location of the vortex perpendicular to the axis of the linear array is kept constant, and several positions parallel to the axis of the linear array (the $\mathrm{x}$ axis) are considered. The results are displayed in Figure 5-28. The position in the y direction of the vortex in each frame of Figure 5-28 is the same as Figure 5-27, 0.1 normalized distance units. The sensors are spaced at a distance of 1/30th (0.033) of a distance unit, chosen as a combination of spacing and array length that is known to result in good localization for a centered vortex at 0.1 units perpendicular distance, as will be discussed shortly. The black curves in each subfigure represent the level sets for which the noise standard deviation is $1 \%, 5 \%$, and $10 \%$ of the maximum pressure magnitude. Also, note that each frame in Figure 5-28 shows the map of the cost normalized for that particular instant. These normalization constants are different from one time to the next, even though the resulting surfaces are plotted with the same color map. In the first few frames of Figure 5-28, the actual vortex position is outside the 45 degree line for all of the sensors implying that they all measure a positive pressure. The nature of the region of ambiguity is not a surprise in these cases, with a broad low-cost region that grows with distance. Between images D and $\mathrm{E}$ in the figure, the vortex passes the $\mathrm{x}$-position 0.166 which takes it past the zero crossing 


\section{Reflected Vortex Lateral Position Effect on Least Squares Cost}
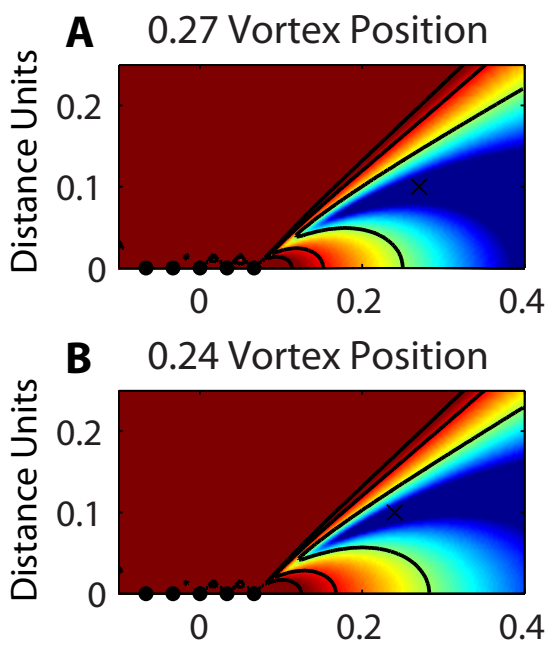

C 0.21 Vortex Position

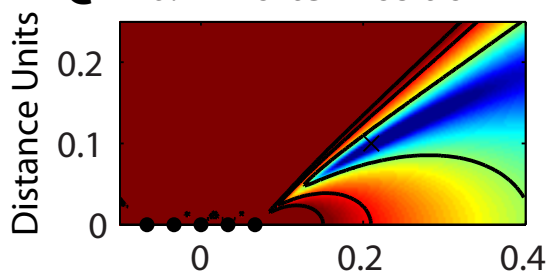

D 0.18 Vortex Position

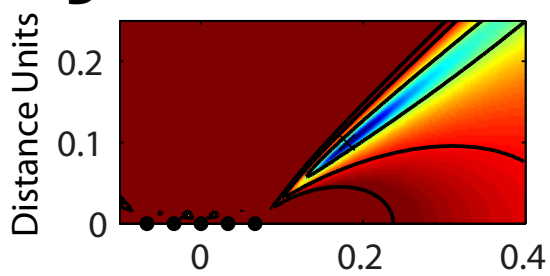

E $\quad 0.15$ Vortex Position

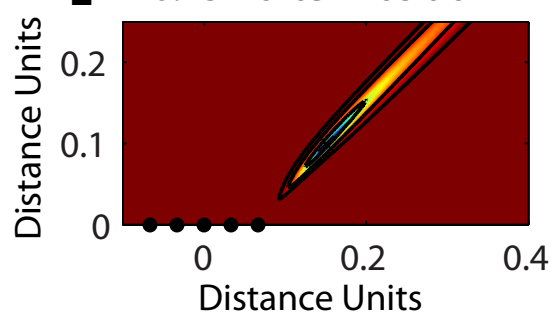

F $\quad 0.12$ Vortex Position

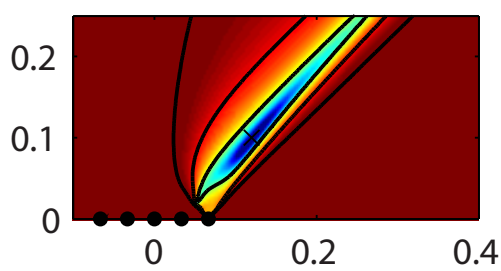

G $\quad 0.09$ Vortex Position

0.2

0.1
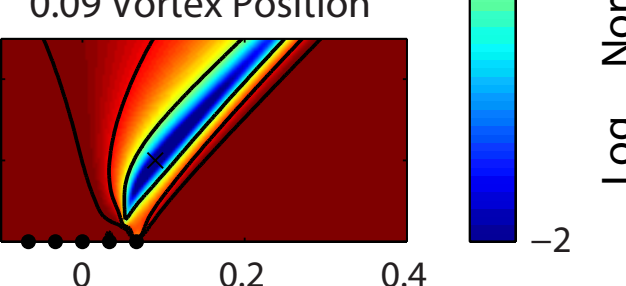

음
H $\quad 0.06$ Vortex Position

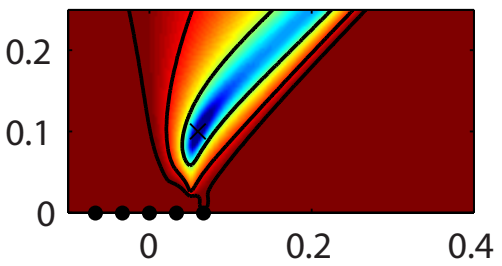

I 0.03 Vortex Position

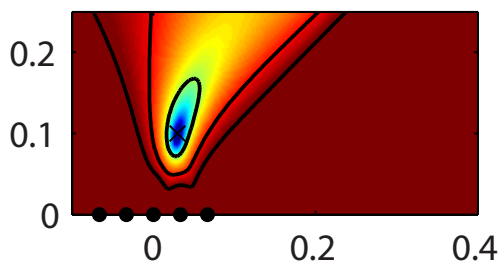

J 0 Vortex Position

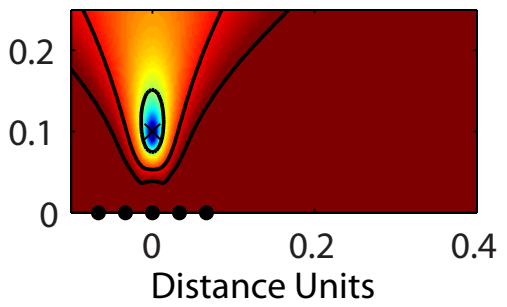

Figure 5-28: Maps of the cost function for a reflected vortex model with the vortex at various lateral locations. The $\mathrm{x}$ in each image with a y position of 0.1 and an $\mathrm{x}$ position labeled in the subfigure title denotes the true location of the vortex. The black lines correspond to level sets generated by the assumption of noise with standard deviations of 1,5 , and $10 \%$ of the maximum measured pressure. 
point of the first sensor. This is marked by a radical reduction in the possible region for the vortex estimate. Interestingly, the region of ambiguity subsequently grows again, until image I. At that point, the vortex is still within the negative region of the first sensor, but has just crossed the 45 degree boundary for the last sensor. Based on this progression, it appears the localization of a vortex under a reflected model as it moves parallel to the sensor array is largely a function of the position relative to the 45 degree lines from each sensor. Also, under the reflected model, it is clear that even under good conditions, it is difficult to locate a vortex that is off to the side of the sensor array. This is particularly true if the vortex is outside the 45 degree line of all the sensors, as in B-D, but it still appears difficult up until the vortex is approximately in front of the first sensor.

\section{Reflected Vortex Distance vs Array Length for Fixed Spacing}
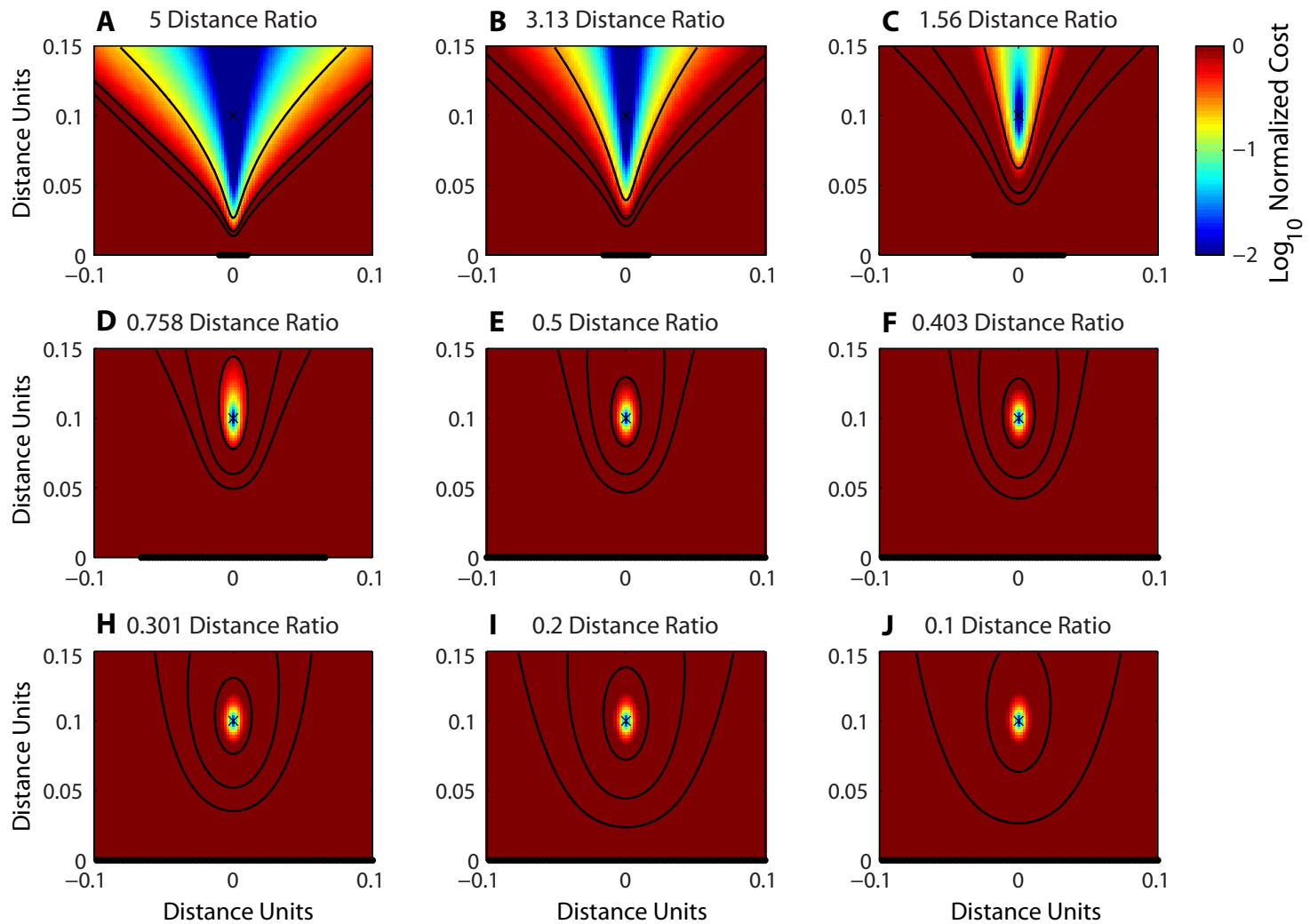

Figure 5-29: Maps of the cost function for a reflected vortex model with an increasing array length but constant sensor spacing. The $\mathrm{x}$ at $(0,0.1)$ in each image denotes the true location of the vortex. The black lines correspond to level sets generated by the assumption of noise with standard deviations of 1,5 , and $10 \%$ of the maximum measured pressure. 
While the cost of a laterally located vortex provides insight into the behavior of the Kalman filter estimation, the main purpose of the instantaneous cost maps is to evaluate the relation between the sensor array parameters, density and length, and the ability to locate a vortex. In order to nondimensionalize the problem, the two ratios of vortex distance to array length and vortex distance to sensor spacing will be considered. Since the lateral location of the vortex is not of interest, the vortex will be centered with respect to the sensor array. Figure 5-29 demonstrates the cost surface for a vortex as the sensor array increases in length while maintaining the same dense sensor spacing. The ratio of the vortex distance to the sensor array length is displayed in the title for each frame. As seen in Figure 5-27 when there is effectively a single sensor, the result is a two valued surface where a large portion of it generates equally valid estimates. The resemblance of this initial state of one sensor to frame A in Figure 5-29 is clear, showing that if the vortex is too far from the sensor array will behave increasingly like a single sensor. Examining the subsequent frames B and C, in which the ratio of the vortex distance to array length decreases moderately, the conelike area of ambiguity condenses to a dominant vertical axis. When taken together with the results from Figure 5-28, it appears that for moderately small arrays (or equivalently moderately large vortex distances) that there is substantial ambiguity in the approximately radial direction from the center of the array. As the array increases further in length in Figure 5-29, the cost function well becomes increasingly smaller vertically, reaching a turning point at around frame $\mathrm{E}$, beyond which the character of the cost map does not change substantially. It is perhaps more than a coincidence that frame $\mathrm{E}$ also corresponds to the point at which the outermost sensors form a 45 degree angle with the vortex against the array line. The widening level sets observed in the last row of frames is largely due to the fact that the number of measurements is increasing while no new information is being added (i.e. the majority of the new sensors are measuring effectively zero pressure). Also, note that all of the cost maps themselves are generated without any noise. Thus transitions in behavior such as at frame $\mathrm{E}$ would be expected to occur much more gradually if using experimental data.

In order to examine the importance of the sensor spacing in the linear sensor array used to track a vortex, consider the same situation as in Figure 5-29, but with a fixed number 
Reflected Vortex Distance vs Array Length for Fixed Number

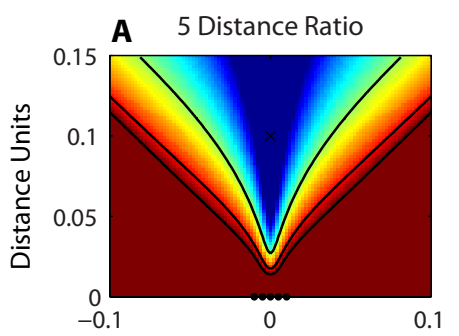

D 0.75 Distance Ratio

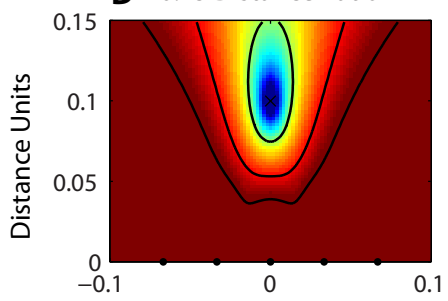

H 0.3 Distance Ratio

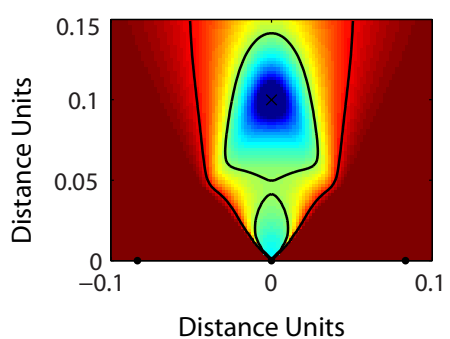

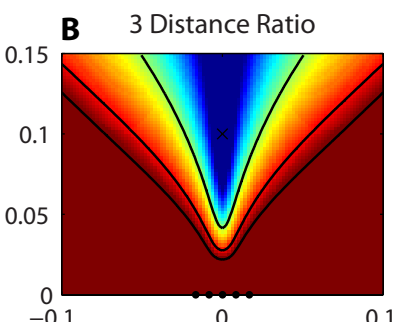

E $\quad 0.5$ Distance Ratio

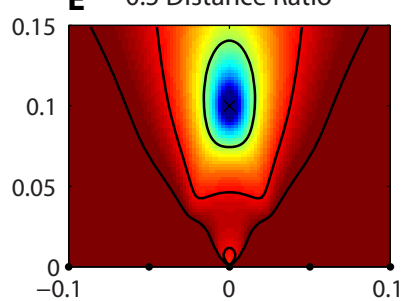

I 0.2 Distance Ratio

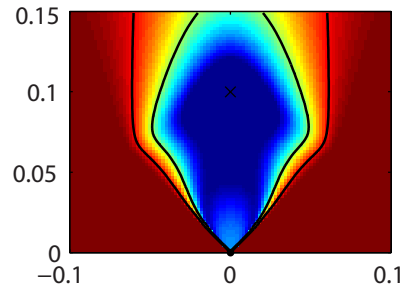

Distance Units

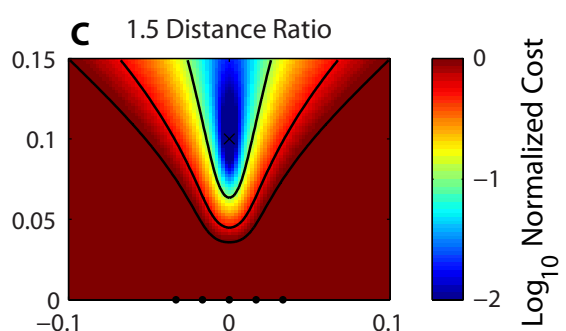

F $\quad 0.4$ Distance Ratio
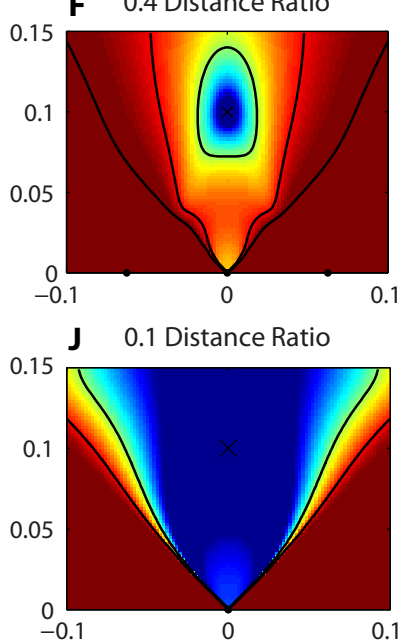

Distance Units

Figure 5-30: Maps of the cost function for a reflected vortex model with an increasing array length and fixed number of sensors. The $\mathrm{x}$ at $(0,0.1)$ in each image denotes the true location of the vortex. The black lines correspond to level sets generated by the assumption of noise with standard deviations of 1,5 , and $10 \%$ of the maximum measured pressure.

of sensors. Figure 5-30 shows the results when the sensor array is limited to five sensors. The black dots along the $\mathrm{x}$ axis represent the position of the five sensors, and the vortex distance to array length ratio is again displayed in the title for each frame. In general, given the substantially fewer sensors involved, cost function for the five sensor case will have a broader distribution of lower costs. Comparing it directly to the results with fixed sensor spacing, it is clear at very large vortex distance to sensor array length ratios, there is little difference in the shape of the cost function distribution. Frames A-C of both simulations have the same global characteristics, though as expected the value of the cost at each point is suppressed (particularly visible in frame C). As the sensor array lengthens, differences between the fixed sensor number and fixed sensor density arrays become more apparent. 
Interestingly, in frames D and E of Figure 5-30, the regions near the true vortex position are effectively undeformed in comparison to the case with constant sensor spacing. Around the periphery, some differences are beginning to show however. In particular, the lower cost region begins to spread towards the array and then outward. Although the limit in the end is the same as the starting point, the area of low cost is originally limited to the immediate vicinity of the closes sensor (frame I). This implies that as a vortex gets too close to a sparse array, the ability to locate it identifying it's closest sensor, is significantly degraded.

\subsubsection{Vortex Pair Model}

The primary practical difference that results from the vortex pair model, not found in the reflected vortex model, is that there is an extra parameter to determine. Following the same line of reasoning as with the vortex strength for the reflected vortex, in order to evaluate the cost of estimating a vortex at a particular location, the cost function is optimized for the vortex strength and vortex separation with the primary vortex at the point of interest. The complex potential for the vortex pair is fundamentally very similar to the reflected vortex (the location of the second vortex is not reflected, but there are still two vortices forming a line perpendicular to the sensor array):

$$
\Phi(z)=-i k \log \left(z-z_{o}\right)+i k \log \left(z-z_{1}\right)
$$

with the additional constraint that if $z_{o}=x+i y$ then $z_{1}=x+i(y+d)$. Therefore, the function for the pressure measurements is still proportional to the strength squared, and, for any combination of position and vortex pair separation, the optimal strength is a straightforward calculation. In order to identify the optimal vortex separation however, the cost function $J\left(d_{o}\right)$ (with $k_{o}$ no longer a free variable, and $x_{o}, y_{o}$ specified) was minimized numerically.

In the case of the reflected vortex model, the 45 degree angle lines emanating from the vortex marked important transitions from positive to negative pressure with respect to the far field. In the case of the vortex pair, where the distance between the vortices is unconstrained, this behavior is no longer present. In the limit of a very large distance 

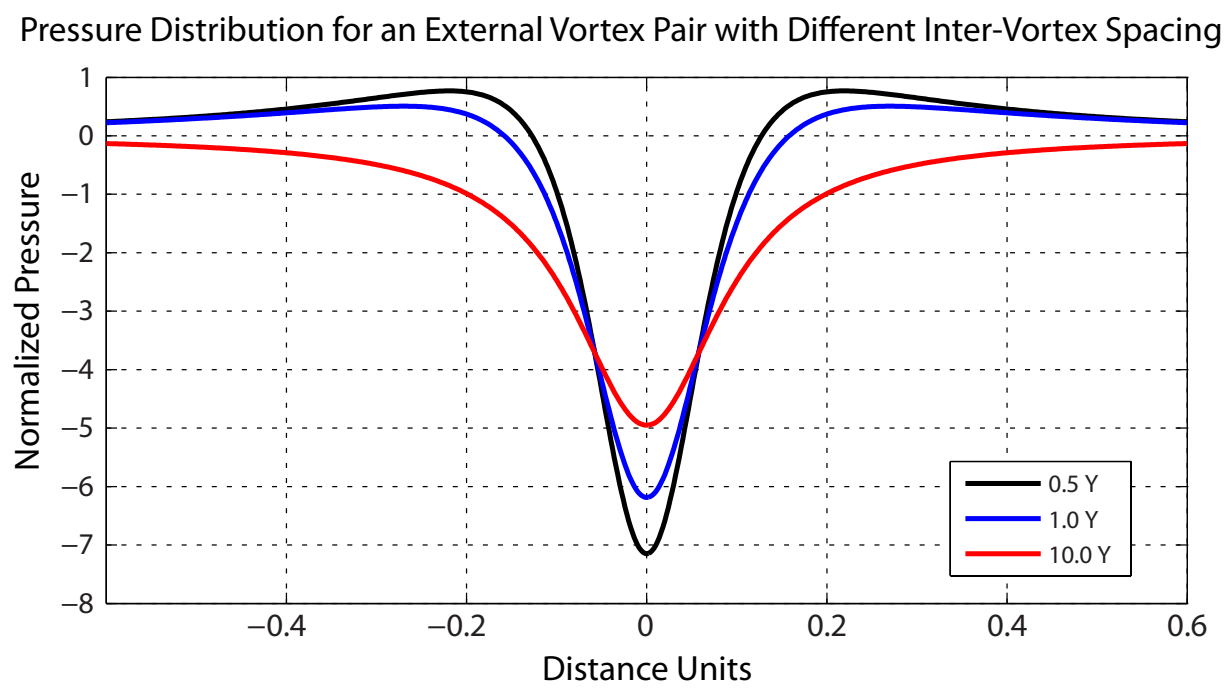

Figure 5-31: The pressure distribution on a linear array due to an external vortex pair. The separation between the vortices is given in the legend with respect to the perpendicular distance between the sensor array and the closest vortex.

between the two vortices, the effect on the sensor array is effectively that of a single vortex. In that case, the pressure distribution on the sensor array is entirely negative and roughly caused only by the local flow velocity. If the secondary vortex is near by, the pressure distribution has the familiar positive pressure regions away from the center of the vortex. Figure 5-31 depicts the pressure distribution along a sensor array line for vortex pairs with three different separations, illustrating the range in distributions. The key implication of the effect of the vortex separation is that, unlike the reflected vortex model, there are no regions in which the position of a vortex is impossible. In the case of a single sensor, a combination of the vortex separation and vortex strength can be chosen for every point in the half-plane in order to exactly reproduce the pressure measurement.

Keep in mind that the freedom in the choice of the vortex separation is to some extent related to the limitations of the instantaneous inversion being considered. The vortex separation is the component that drives the speed at which the vortex pair translates, and therefore a time history of pressure measurements would provide some independent information on the distance between the vortices. This implies that the vortex distance parameter will not be as freely varying from the point of view of a Kalman filter. 


\section{Vortex Pair Lateral Position Effect on Least Squares Cost}

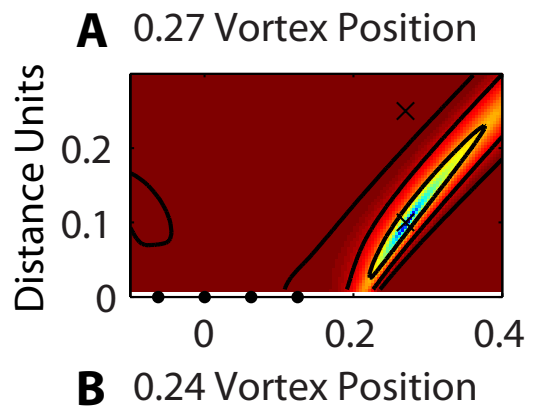

F 0.12 Vortex Position

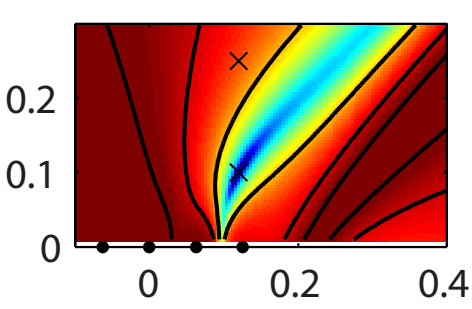

G 0.09 Vortex Position
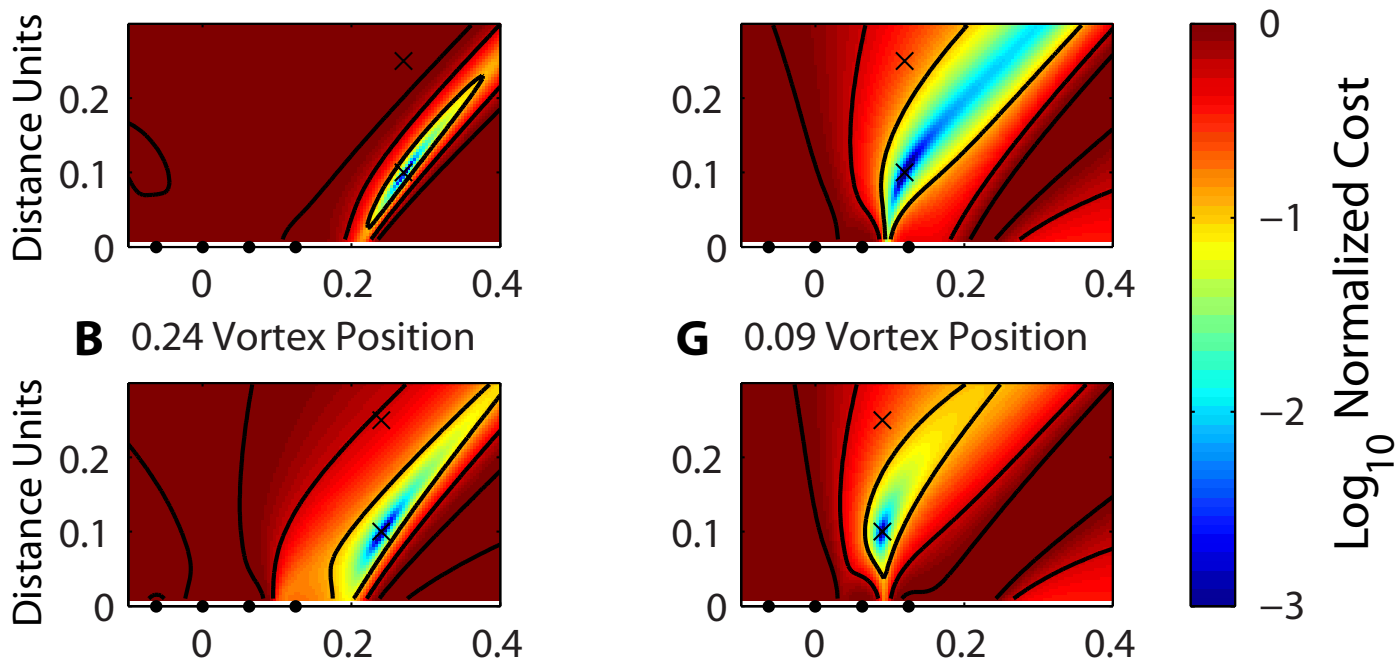

C 0.21 Vortex Position

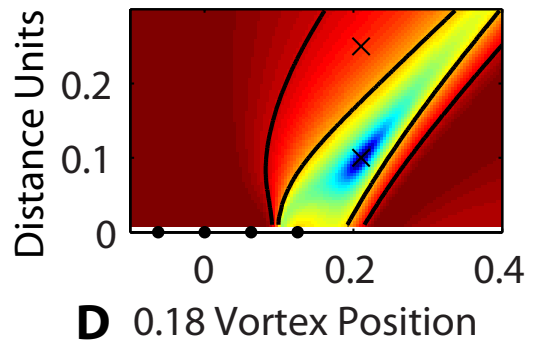

H 0.06 Vortex Position
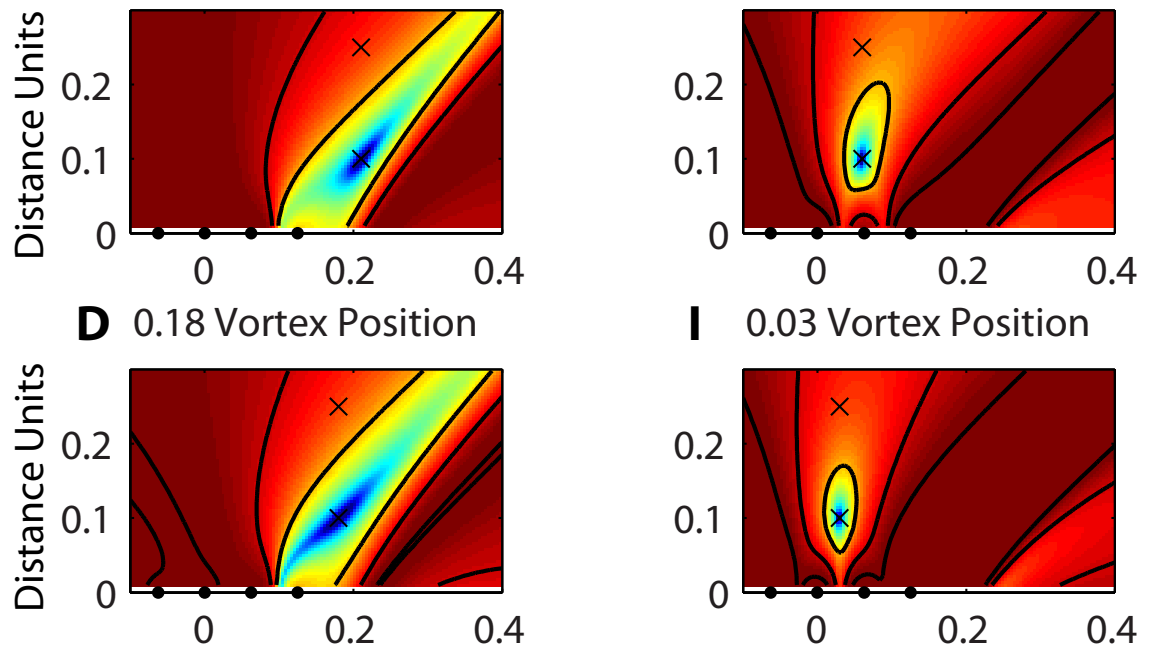

I 0.03 Vortex Position

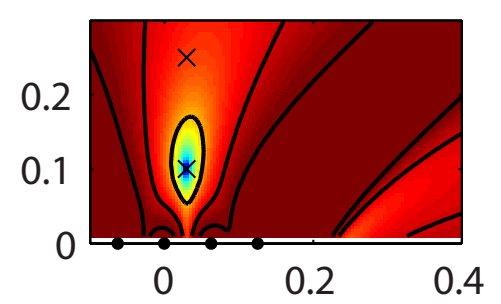

E 0.15 Vortex Position

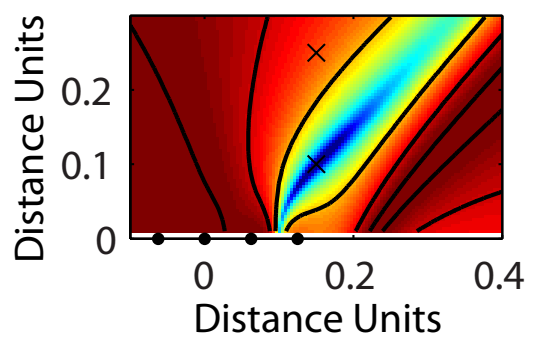

J 0 Vortex Position

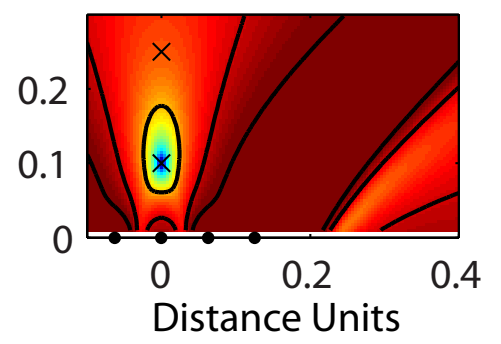

Figure 5-32: Maps of the cost function for a vortex pair model with the vortices at various lateral locations. The $\mathrm{x}$ in each image denotes the true locations of the vortices. The black lines correspond to level sets generated by the assumption of noise with standard deviations of 1,5 , and $10 \%$ of the maximum measured pressure. 
Despite the substantial differences between the models, there are still numerous similarities in the way the cost function is distributed under the various conditions. First, Figure 5-32 shows the cost function maps that correspond to a vortex at several lateral locations with respect to the sensor array, with the frames corresponding exactly to those in Figure 5-28. At the initial stages (up to frame $\mathrm{H}$ ), the regions of low cost are very elongated and generally aligned with the radial direction from the center of the array. Much like in the reflected vortex case, the region of minimum cost shrinks and is well localized in both $\mathrm{x}$ and y directions as the true vortex reaches the center of the sensor array. While this broadly matches the results for the reflected vortex model, there are numerous differences in the details that imply that the behavior of estimation techniques may differ for the two models. One of the most important differences in this progression is that there is not just a single low-cost lobe for the vortex pair model. As visible in frames H-J, there appear to be local minima far off to the side from the main low-cost region. This is potentially troubling for the purposes of estimation, as it implies that with a very poor set of initial conditions and the wrong circumstances, the Kalman filter may converge to an incorrect solution. This was not observed experimentally, so it is possible that it is not a serious concern when considering multiple timesteps. A second difference in comparison to the reflected vortex results is that the main well in the cost function is much better localized when the vortex is far to the side. This discrepancy is most apparent when comparing the first frame of each figure. Although the cost map broadens in subsequent frames for the vortex pair, frame A of the vortex pair most resembles frame $\mathrm{E}$ of the reflected vortex results. This implies that the Kalman filter or another estimation technique might begin to converge to the true vortex state earlier for a vortex pair. In addition, it appears that the region of low cost shrinks more rapidly for the vortex pair model as the vortex pair nears the center of the array. Another point of interest is that, unlike the case with the reflected vortex model, the regions of low cost for the vortex pair extend completely to the sensor array. This is particularly true for frames D and E, where it appears that an estimate that is directly between the two closest sensors to the true vortex would be able to generate a reasonably close approximation to the measured pressure.

The maps of the cost function that correspond to sensor arrays of increasing size but 
constant sensor spacing (Figure 5-33) demonstrate a very similar behavior to that of the reflected vortex model. Interestingly, when the length of the array is very small (frame A) the distribution of the cost function still generates a cone-like region of low cost extending vertically, much like in Figure 5-33A. This region of ambiguity narrows quickly into a vertical strip (frame C, D) before becoming localized to a small ellipse. Despite the different model, it appears that the map of the cost function is relatively unchanged for ratios larger than 0.5 of the distance to the first vortex versus the array length. This is the same point at which the behavior changed for the reflected vortex model.
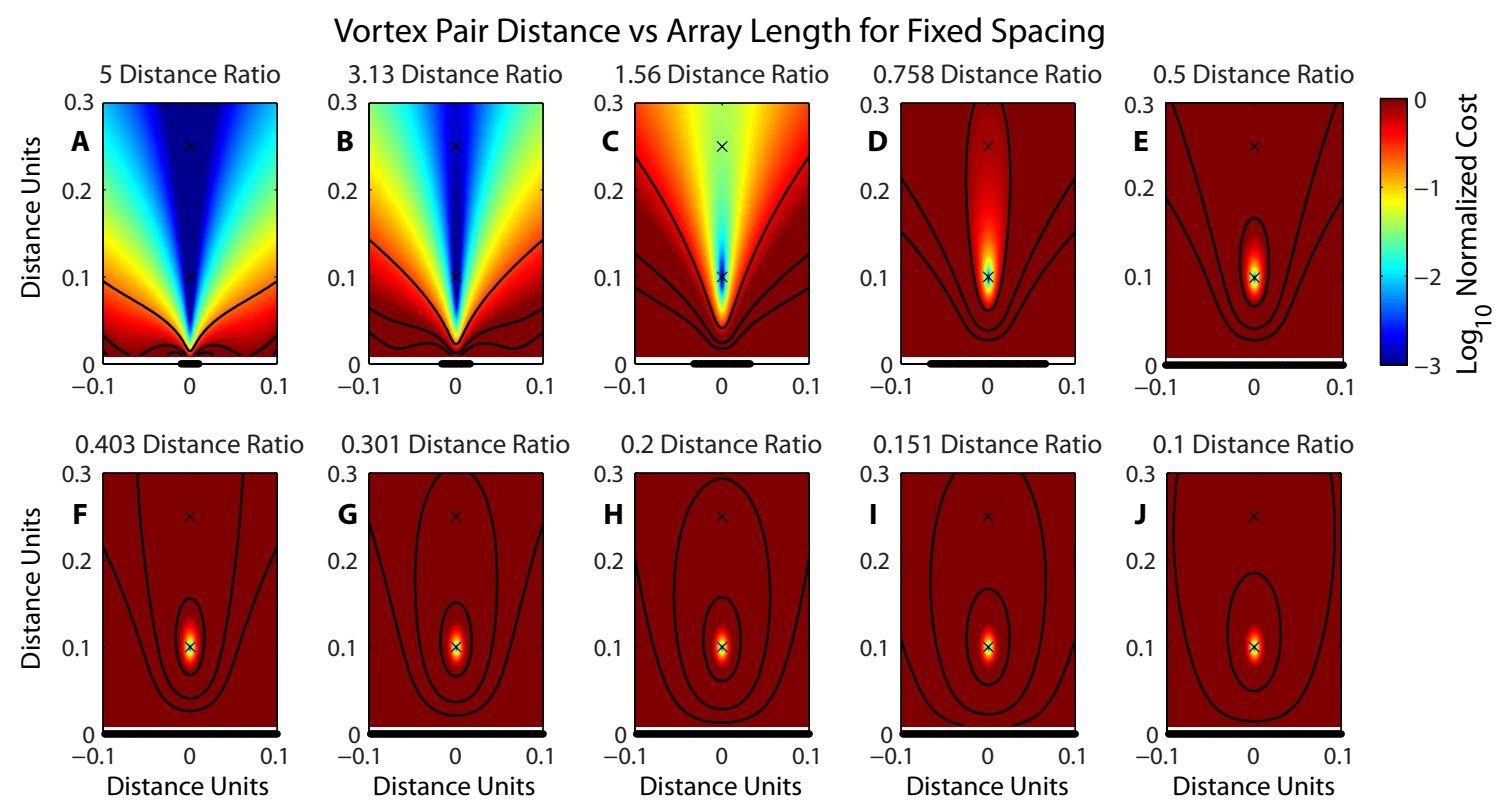

Figure 5-33: Maps of the cost function for a vortex pair model with an increasing array length but constant sensor spacing. The $\mathrm{x}$ in each image denotes the true location of the vortex. The black lines correspond to level sets generated by the assumption of noise with standard deviations of 1,5 , and $10 \%$ of the maximum measured pressure.

While the broad similarities between the reflected vortex model and the vortex pair model remain in the case of the lengthening array with constant number of sensors, there are more differences in the details. Generally speaking, following the progression in Figure 5-34, at a high distance-to-length ratio the estimation begins with low sensitivity in the $\mathrm{y}$ direction, and at a low distance-to-length ratio the estimate has low sensitivity to position within a small region about the closest sensor. However, the areas for a low cost estimate 
for the vortex location generally extend much closer to the sensor array for all ratios when compared to the reflected vortex model. Much like what was observed when the considering the question of lateral vortex position, tendrils of low cost extend all the way to the sensor array between some of the sensors (particularly in frames E-G). These tendrils of low cost begin to become visible quite early, at a ratio of vortex distance to sensor spacing of 3. At the extreme depicted in the figure, where the sensor array was very sparse in relation to the vortex position (frame $\mathrm{J}$ ), it is interesting to note that region of low cost is still quite limited in the y direction. This is true in comparison to the results of the reflected vortex, but perhaps unsurprising given the observations in Figure 5-32 which found the cost map surprisingly narrow for a vortex at a distance. From the point of view of interpreting the implications on vortex tracking for a Kalman filter or another estimation technique, these results imply that for a sparse sensor array, or for a vortex overly close to the array, the results will possibly underestimate the distance of the vortex.
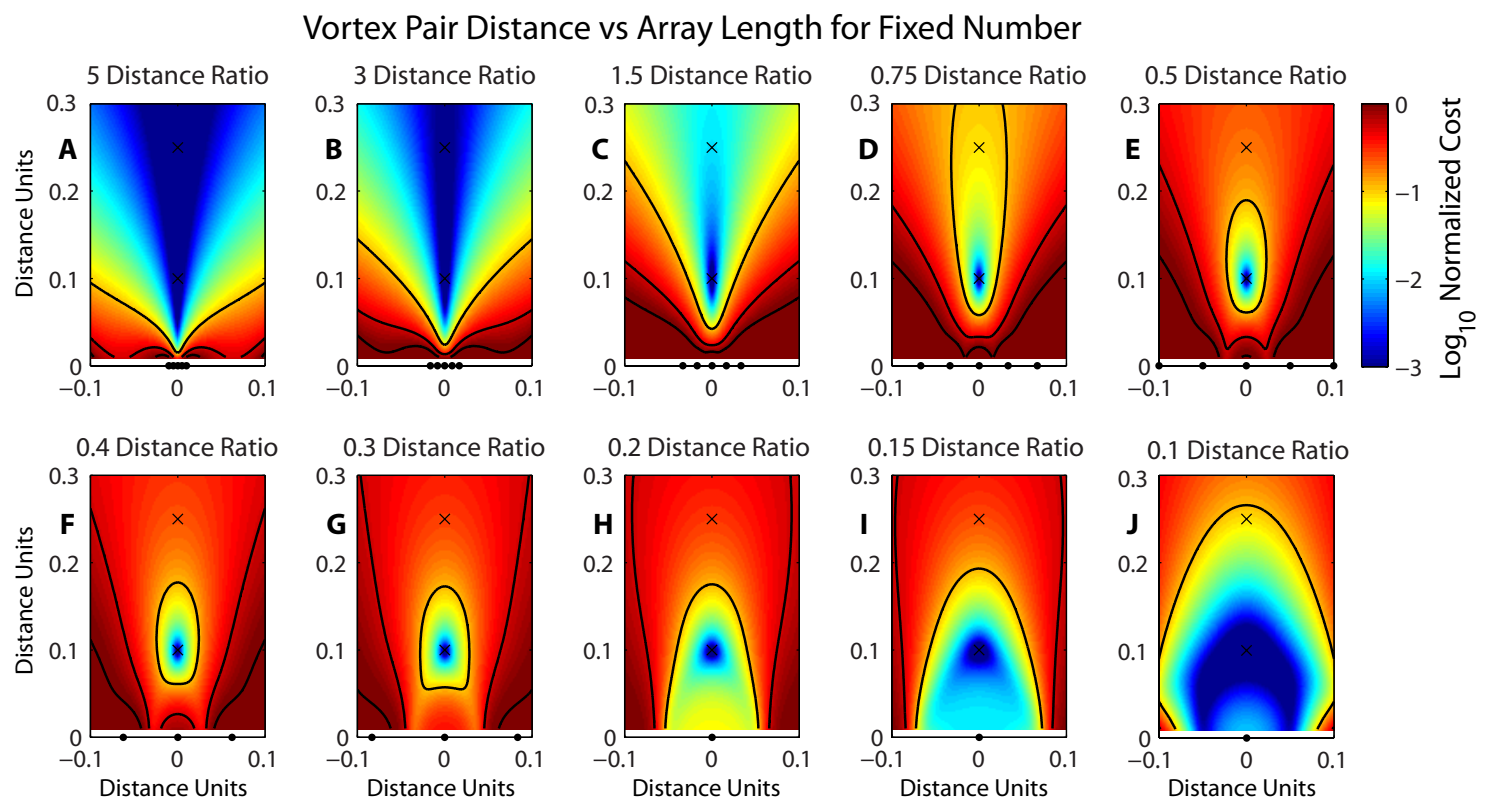

Figure 5-34: Maps of the cost function for a vortex pair model with an increasing array length fixed number of sensors. The $\mathrm{x}$ in each image denotes the true location of the vortex. The black lines correspond to level sets generated by the assumption of noise with standard deviations of 1,5 , and $10 \%$ of the maximum measured pressure.

From the point of view of designing a linear sensor array for the purposes of tracking a 
nearby vortices, there are some general guidelines that can be extracted from this analysis. Fundamentally, these guidelines relate the array parameters to the region of interest in which vortices are to be tracked. First of all, regardless of whether the sensor array is to be mounted on a small body or a large flat hull (determining which model to use), there is little to be done in extending the accuracy of estimation out to the sides of a linear array. The only solution is to increase the length of an array so that the entire area of interest is directly in front of the sensor array. This is clear from the figures depicting the cost function distribution as a vortex moves parallel to the sensor arrays. Apart from this effect, the outer range of interest should be used to determine the length of the array, with the array length at least twice as long as the perpendicular distance to the vortex. The density of the array should be determined based on the minimum perpendicular distance of interest for vortices. A rough guideline for the minimum distance would be to maintain the sensor spacing at less than half the expected minimum perpendicular distance of the vortex. While the type of ambiguity differs for the two different models, the rough spatial guidelines for a sensor array are the same regardless of the shape on which the array is mounted.

It is important to note that this analysis effectively assumes that the strength of the vortex is sufficient to generate measureable changes in pressure at the location of the sensor array. In reality, the outer range of vortex detection is also limited by the noise floor of the pressure sensors. Beyond some point, there will be no signal detected by the pressure sensors and vortex tracking will not be possible. The effect of vortices with moderately small strengths on vortex detection can be inferred from the behavior of the level curves in Figures 5-28-5-34 which represent increasing sensor noise levels.

\subsection{Discussion}

One of the discussed applications which could utilize vortex tracking via a pressure sensor array, as discussed in the previous section, is energy extraction via flapping foils. In this scenario, the interaction between vortices and the foil is important in two ways. First

and most critically, the leading edge vortex that forms from motion of the foil can lead to substantially different wake structures and energy efficiency [71]. Secondly, a similar 
situation can occur through interactions between a foil and an externally generated vortex that drifts past the foil from upstream. In this latter case, the interaction between the foil and vortex can lead to substantially increased or decreased lift, affecting the performance of the foil. This has been demonstrated theoretically by the work of Streitlien [72] and experimentally by Gopalkrishnan [30] where the relative positions were altered via the phase between foil oscillation and vortex shedding. In this case, identifying the relative location of the external vortex to the foil is critically important for predicting the type of affect that will result from the interaction [30]. In contrast, the self generated vortex is a result of the motion of foil and can therefore be predicted beforehand, though changes in the environment may still lead to alterations that would require feedback. The application of tracking externally generated vortices near a foil, and their effect on the lift of the foil, provides a good reference for examining the performance of the vortex tracking results.

\begin{tabular}{|c|c|c|}
\hline & Bias & Standard Deviation \\
\hline $\mathrm{X}$ & $-0.36 \mathrm{~mm}$ & $7.7 \mathrm{~mm}$ \\
\hline $\mathrm{Y}$ & $0.43 \mathrm{~mm}$ & $3.9 \mathrm{~mm}$ \\
\hline $\mathrm{K}$ & $-46 \mathrm{~mm}^{2} / \mathrm{s}$ & $190 \mathrm{~mm}^{2} / \mathrm{s}$ \\
\hline
\end{tabular}

Table 5.1: Error statistics for tracking vortices from a streamlined body. The statistics are calculated while the vortex is in front of the sensor array, allowing for a brief window of convergence.

First, a sense of the error statistics can be extracted from the experimental results with a full set of functioning sensors on a streamlined body and dense PIV time sampling. These results are shown in Table 5.1. It is interesting to note that the standard deviation is larger in the $x$ direction, which was not necessarily clear from the earlier figures due to the different scales. The relative position of the vortex to the foil is the important component here, as the strength will affect the quantity of the extra lift possible, but not the preferred path of the vortex in order to take advantage of it. Therefore we will not focus on the strength.

These results correspond to a vortex approximately $5 \mathrm{~cm}$ away from a $19 \mathrm{~cm}$ long array with $6.35 \mathrm{~cm}$ spacing between sensors, which places it within the range of target array parameters identified for a vortex of that distance. As a result, the error should scale with the array size, assuming the relative distance of the vortex to the array length remains the 
same. This also assumes that the pressure measurements are sufficiently large with respect to the noise floor such that reliable measurements are available, as was the case in the full scale experiment. In this situation the standard deviations normalized with respect to the array length are 0.04 units in the $x$ direction and 0.02 units in the $y$ direction

A similar set of results can be obtained from the experiments with the sensors embedded in a flat plate (Table 5.2). As expected, there are significant differences in the behavior of the Y estimate under these conditions, associated with the corruption of the data due to secondary vorticity observed in the PIV. Since a foil can be reasonably approximated as two dimensional, it is likely to influence the vortex in a manner closer to the experiments with the flat plate as opposed to the streamlined body, and thus the error statistics of the flat plate results may be more applicable. In this case, the error in lift due to the ambiguity in the $x$ direction is comparable to before with the streamlined body, but there is an underestimation of the $y$ position that will additionally affect the lift estimate, in addition to the increased standard deviation in the $y$ direction. Although the density of sensors is different for the flat plate experiments, the location of the vortex with respect to the array length is comparable to that of the streamlined body case. The normalized values are therefore 0.04 units standard deviation in the $x$ direction and 0.04 units standard deviation in the $y$ direction, 0.08 units of bias in the $y$ direction.

\begin{tabular}{|c|c|c|}
\hline & Bias & Standard Deviation \\
\hline $\mathrm{X}$ & $2.9 \mathrm{~mm}$ & $7.1 \mathrm{~mm}$ \\
\hline $\mathrm{Y}$ & $-15 \mathrm{~mm}$ & $7.9 \mathrm{~mm}$ \\
\hline $\mathrm{K}$ & $-24 \mathrm{~cm}^{2} / \mathrm{s}$ & $14 \mathrm{~cm}^{2} / \mathrm{s}$ \\
\hline
\end{tabular}

Table 5.2: Error statistics for tracking vortices from a flat plate. The statistics are calculated while the vortex is in front of the sensor array, allowing for a brief window of convergence.

The response of a foil to a vortex passing nearby can be modeled using a potential flow model in which the wake of the foil is simulated by a series of discrete vortices, satisfying the Kutta condition at the trailing edge each timestep. This model was implemented according to the work by Streitlien et al. [73, 72]. For this analysis, the pressure sensor array will be simulated over the front half of the upper foil, as for any practical purposes, the vortex would need to be located early in the interaction in order to extract the maximum 


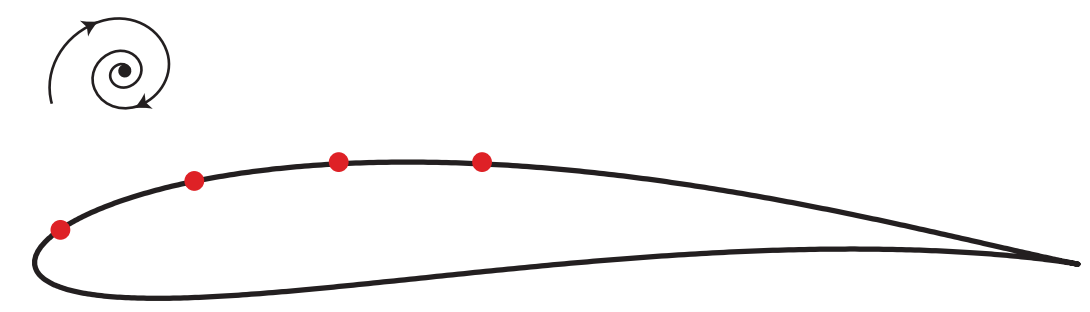

Figure 5-35: Simulated setup for a pressure sensor array similar to the one on a streamlined body being applied to a foil in order to track an external vortex.

benefit. In addition, when the vortex is near the trailing edge of the foil, it interacts with the vortex trail being shed. This means that the history of the vortex is important at that point, and a simple sensitivity analysis becomes more complex. In the case of the four sensor implementation used in the streamlined body experiments, a depiction of the sensor array on the Joukowski foil used in the simulation is shown in Figure 5-35. Both the lift and the moment on the foil will be considered, using a full unsteady model that takes into account the motion of the external vortex.

The focus is on the location of the vortex, but the strength of the external vortex needs to be specified for the simulations. In this analysis, the external vortex has a circulation equal to half that of the circulation about the foil required to maintain the Kutta condition when there is no external vortex. All of the distances and positions will be normalized by twice the chord length of the foil. Additionally, all of the lifts will be normalized by the natural lift of the foil in the absence of a vortex. In the case of the moments, the moment will be normalized by a nominal moment equivalent to that required to rotate the foil at a constant angular velocity (1 radian per second) in a steady flow but with no vortex present.

\section{Lift}

With respect to the lift without a vortex present, the vortex depresses the lift when it is ahead of the foil and augments it when the vortex is directly above the foil(Figure 5-36). The maximum change in the lift with respect to the lateral position occurs in the rough location leading edge of the foil. At this point, the sensitivity of the lift is 1.64 normalized lift per normalized $x$ position. This implies that the error in the lift estimate due to ambiguity in 

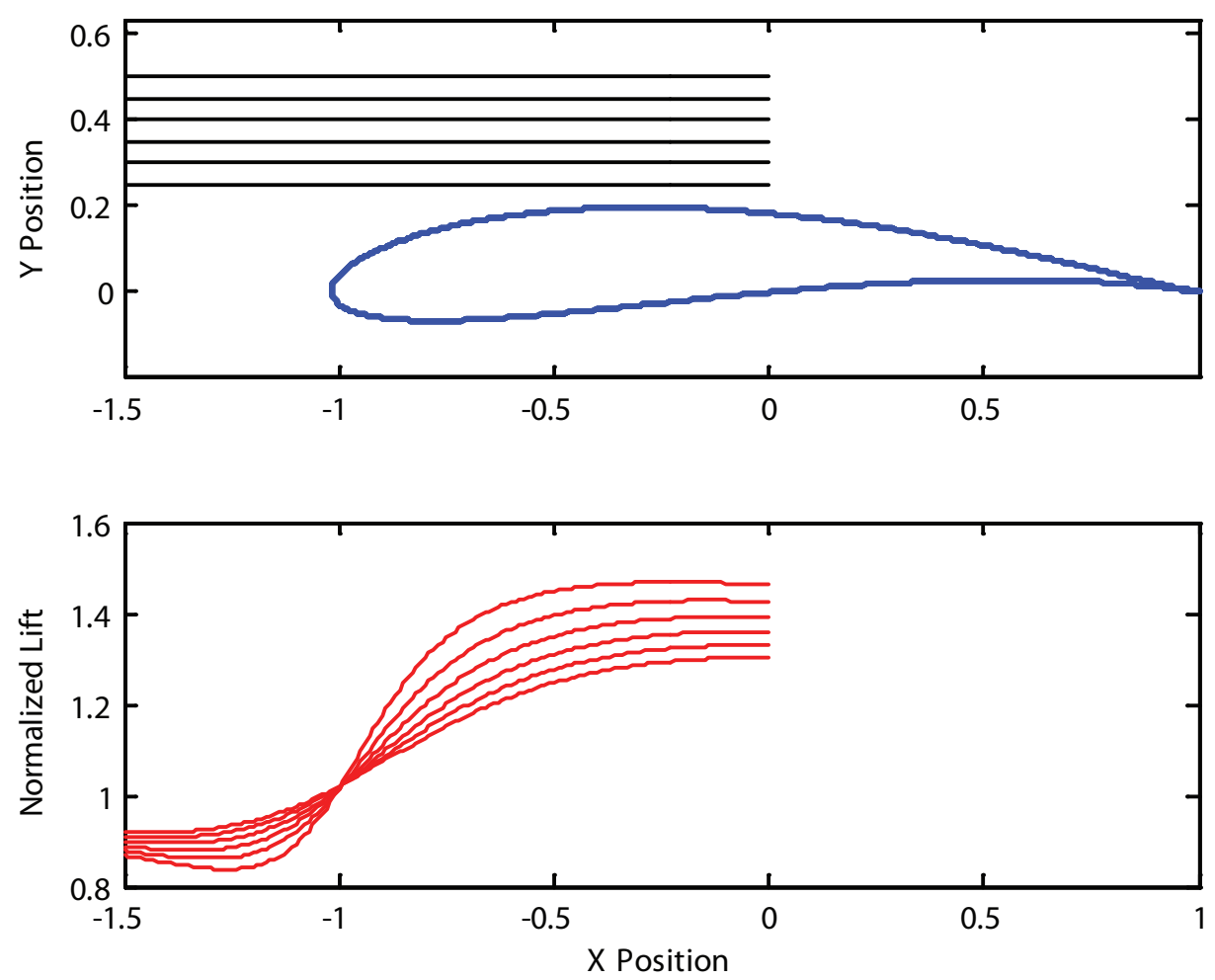

Figure 5-36: Dependence of foil lift on the position of an external vortex. In the top subfigure, the foil is plotted with horizontal lines indicating the range of positions of the external vortex. The lower subfigure demonstrates the effect of a vortex at the positions marked in the top subfigure on the normalized lift of the foil. 
the $x$ position estimate in the lateral line tracking from a streamlined body would have a standard deviation of at worst approximately $6.5 \%$ of the lift without a vortex present. For comparison, the maximum effect of the vortex is approximately $50 \%$ of the lift without a vortex.

The lift decays monotonically as the vortex increases in $y$ position from the surface of the foil (assuming a positive lift contribution). Consider a location along the foil where the overall change in lift is the greatest ( $x=-0.6$ in Figure 5-36). In this case, working with the maximum sensitivity (1.5) near the surface does not make sense as it is not in the estimation range of the array. Instead, using a distance in proportion to the array length, the sensitivity is approximately 0.59 normalized lift units per normalized distance unit in the y direction. Based on the error in estimating the vortex y position experimentally from a streamlined body, this corresponds to a standard deviation in the lift estimate of approximately $1.2 \%$ of the lift when no vortex is present. Using a locally linear approximation to the lift's dependence on position, the combination of ambiguity in both $x$ and $y$ results in an estimated standard deviation of approximately $6.6 \%$ of the lift with very little bias.

In the case of the experiments involving tracking vortices with interaction between the vortex and the sensing body, lift estimate standard deviation will be larger due to the increased ambiguity in the $y$ direction, but there will also be a component of the error due to the bias in the $y$ position estimate. The results in the $y$ direction correspond to a lift estimate with a $4.6 \%$ lift bias and a standard deviation of $2.6 \%$ of the lift with no vortex present. It is clear from this analysis that the lateral $(x)$ position of the vortex is of larger importance for predicting the lift felt on a foil, even when the increased variability in the $y$ estimate due to the interactions with the foil surface are taken into account. Combining the results for both the $x$ and $y$ directions, the estimated error in the lift would have a bias of $4.6 \%$ and standard deviation of $7 \%$.

In either case, taking the streamlined body error statistics or that of the flat plate, the error in estimating the lift due to nearby vortex is small compared to the benefit that the vortex is able to provide for the foil. This implies that the vortex could be tracked sufficiently well to utilize that vortex in increasing the foil lift, with a good prediction of the effects on the foil. 


\section{Moment}

The moment that a vortex produces on a flapping foil is of interest because it might be able to be utilized to reduce the energy expenditure in driving the foil. Since flapping foils for extracting energy from flow are frequently actuated in pitch, any reduction the torque necessary to actuate the foil would automatically translate to improved efficiency. In addition, when the vortex is upstream of the pivot point being considered, the vortex generates a moment that counters the desired motion of the foil (assuming the vortex is towards the outside of the foil), which would result in a waste of energy if the pitch were actuated at that time.

Figure 5-37 illustrates the dependence of the foil moment on the position of the external vortex. As in the model by Streitlien, the moments are computed about a point at the midchord of a cambered foil. As mentioned already, the moment is normalized by the torque required to rotate the foil at a constant angular velocity of 1 radian per second in a steady flow in the absence of a vortex. Also note that the moment depicted in Figure 5-37 is positive when clockwise.

Similar to when considering the lift, this evaluation will be restricted to the front half of the foil, where the interaction between the externally generated vortex and the trailing edge vortices is minimized. Not surprisingly, the moments generated in response to the vortex are reduced when the vortex is directly over the foil, due to the decreased moment arm. The maximum sensitivity to the $y$ position occurs in front of the leading edge of the foil, while the maximum sensitivity to the $x$ position occurs slightly behind the leading edge.

In the case of the sensitivity in the moment estimate with respect to the $x$ position of the vortex, the maximum sensitivity for a vortex near the surface is approximately 3.55 moment units per distance unit. Although this location is too close to the foil surface for adequate tracking given the array parameters, it provides a good upper bound for the error in estimating the moment. Both the streamlined body experimental results and the experimental results with a vortex interacting with a flat plate provided similar error statistics for the $x$ position estimate. The corresponding error in the moment estimate would be have standard deviation of approximately $14.2 \%$ of the reference moment. 
As with the lift, the moment generated by a vortex out in front of the foil decays monotonically as the vertical separation increases. At particularly small $y$ positions, there is a peak in sensitivity, but the location results in a vortex that will pass very close to the surface of the foil. For a position upstream of the foil that is a reasonable distance for tracking the vortex and that corresponds to the vortex path crossing the appropriate estimation region, the sensitivity of the moment to position in the $y$ direction is approximately 2.3 moment units to distance units in the $y$ direction. This corresponds to an estimation error in the moment of approximately $4.6 \%$ of the reference moment in standard deviation when based on the results of the streamlined body vortex tracking. When one considers the possible effect of the vortex-body interactions, the corresponding results would be a $21 \%$ bias with a standard deviation of $11 \%$ of the reference moment.
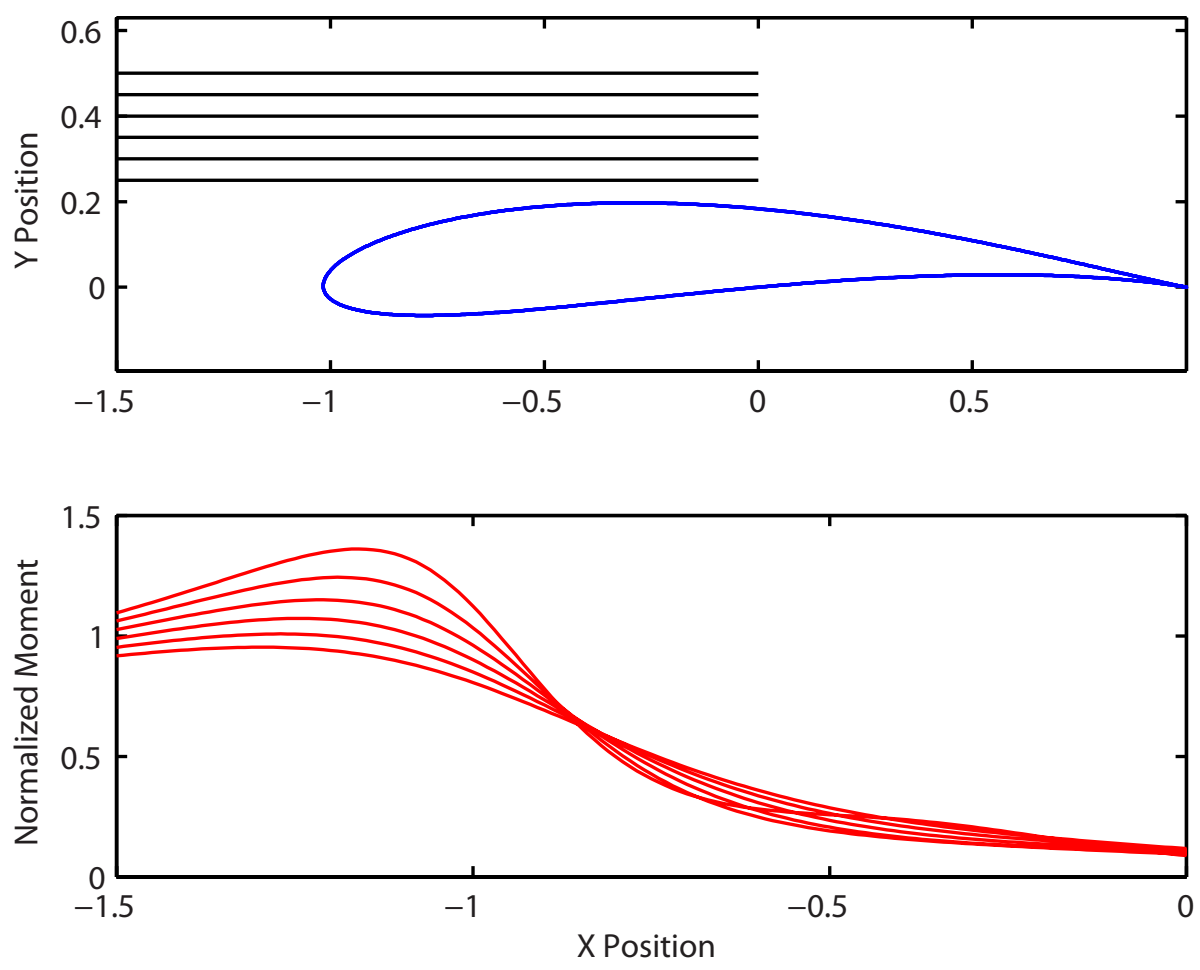

Figure 5-37: Dependence of foil moment on the position of an external vortex. In the top subfigure, the foil is plotted with horizontal lines indicating the range of positions of the external vortex. The lower subfigure demonstrates the effect of a vortex at the positions marked in the top subfigure on the normalized moment of the foil.

Compared to the lift estimates, the bias in the $y$ estimates of the vortex position that 
occurs due to the foil-vortex interactions results in more substantial error. However, in the context of the overall moment that vortex can cause to act against the desired pitching motion (about $80 \%$ ), this still provides information which can be used to predict the effects of the interaction on the foil. In addition, the nature of the bias causes the estimated adverse affects to be exaggerated, which will not lead to an excess expenditure of energy.

\subsection{Conclusions}

One of the primary motivations for identifying in detail the state of a vortex is its influence over foils and control surfaces. The vortex is a persistent fluid structure which affects the pressure distribution over the surface of nearby objects. By nature, a lateral-line-like sensor array of the sort used in this chapter already gives the pressure distribution over a region of the body surface. However, by identifying the vortex itself instead stopping at the pressure distribution over a measured line, there are two substantial advantages. The first of these is that using a limited sensor array to develop an accurate model of the local flow removes the need to instrument the entire surface with pressure sensors. Instead, a small artificial lateral line provides a description of the flow that can be extended to provide the pressure distribution over the entire nearby surface. Secondly, by knowing the state of the vortex, it is possible to predict how the pressure will change in the near future due to the motion of the vortex (via advection and interaction with the body) and thus plan for how the vortex will affect the structure. The results of the vortex tracking on the streamlined body and the flat body demonstrate that a small pressure sensor array is sufficient for this purpose, and the simulated results on array parameters give a starting point for the adequate design of such a sensor array.

One important conclusion from the experimental results on estimating vortices with the streamlined body is that in two dimensions it is a much closer approximation to use an invisible body than a solid one. This has been remarked previously (for example see [65]). However, it also implies that studies such as Ren and Mohseni [66] overestimate the effect of the fish body on the flow. In addition to the effects on the global flow, this lack of interaction appears to extend to the question of generating secondary vortices due to the 
interactions between the primary vortex and the sensing body. This effect was observed in the case of the flat plate experiments with substantial implications on vortex tracking, but was completely absent in the streamlined body experiments. The result shows that the simplified two dimensional inviscid flow model fits very well for fish-like small streamlined bodies, and the corresponding vortex estimates tracked the nearest vortex accurately under a variety of conditions.

In the context of fish, the results of the experiments described in this chapter on tracking vortices from a streamlined body clearly indicate that it is possible to obtain specific information on the state of a nearby vortex based solely on a lateral-line-like sensor. Previously, experiments with live fish have tied the lateral line to energy-saving gaits that require interaction with vortices $[46,43]$, but it has been unclear how much information was being extracted via the lateral line and how it is used. While the demonstration of vortex tracking in the streamlined body experiments do not answer this question, they do make it clear that it is possible that a fish obtains information about individual vortices, as opposed to only global characteristics such as shedding frequency.

It is still questionable whether it is possible to extract specific information for two or more vortices that are not spread out along the length of a pressure sensor array. The results with a streamlined sensing body, in which a vortex pair was used as the stimulus, were not able to accurately reconstruct the position of the second, further, vortex. It was found that the position of this vortex was insensitive to the pressure measurements, and therefore did not converge in the time (roughly 2 seconds) of available measurable signals. While the relative insensitivity is unlikely to change, it is likely that with a larger number of sensors the estimation of the second vortex would improve since it effectively improves the reliability of the measurements. In addition, a longer sensor array that therefore measures the vortex pair for a larger period of time would likely improve the final estimate of the vortex pair.

This extra difficulty in tracking multiple vortices does not necessarily hinder a fish in a Karman vortex street. During the energy saving swimming, the fish swims between the alternating vortices. Besides each vortex being spaced out in the direction of the lateral line, this also means only a single vortex is is directly in front each face of the lateral line 
at a time. It is likely that the vortices on either side of the fish can't be treated completely independently from the point of the lateral line, but it will not be the same as the vortex pair scenario considered here.

The results with the sensor array embedded in a flat plate have shown that it is similarly possible to track an individual vortex when interactions with the sensing body are more substantial. However, it was clear that under these conditions secondary vorticity is a concern and will affect the estimation. The affect appears to be manageable in that it causes an underestimation in the $y$ position and overestimation in the strength, but does not cause the problem inversion to fail into an unrecoverable state. Based on the simulation results considered in the later part of the chapter, the sensor array is not dense enough with respect to the $y$ distance of secondary vortices as they form. With a denser sensor array, it is likely that the primary vortex would be tracked more accurately using the same method.

Besides the relevance to tracking externally generated vortices near a hull, the resilience to secondary vorticity of the estimation based on a sensor on a flat plate implies that a similar approach could be extended for self-generated vortices. Self generated vortices, such as the leading edge vortex in a foil, generate from the separation of the flow on a body and are therefore always in close proximity to the sensing surface. As long as the body of the sensors is taken into account, it appears that any secondary vorticity that occurs in the interaction between the vortex and surface can be considered a secondary issue that can perhaps be addressed by increased density.

When the vortex estimation results are viewed from the context of specific applications, it is possible to evaluate the degree of error in the estimation. In this case, the error in the position estimate of the vortex tracking was analyzed in the context of generating lift and moments on a foil. It was found that the error in the lift prediction due to the ambiguity in vortex position is substantially less than the maximum lift the can be generated between the foil and the external vortex, leading to the conclusion that the vortex tracking could be used to improve the performance of a flapping foil energy extraction device. A similar situation was observed with respect to the moment that is generated on a foil due to an external vortex, though the effect of the bias from secondary vorticity has a more substantial effect. 


\section{Chapter 6}

\section{Conclusions}

\subsection{Overview}

The fish lateral line organ presents a completely novel approach to sensing and interpreting the local flow environment in the non-biological world of AUV's and ships. Sensors based on the lateral line have the potential, based on biological observations, to fill a gap left by other sensory systems in complex environments and enhance the abilities of vehicles to avoid collisions, identify unknown objects, and react to local flow. However, much is still unknown about the workings of the lateral line and how well it would translate to man-made vehicles.

The goal of this thesis has been to evaluate the realizable potential of lateral-line inspired sensors in two specific applications: tracking and identifying a cylinder and tracking a vortex. Due to the lack of information about the actual lateral line processing, the first essential task consisted of identifying appropriate estimation techniques for extracting the desired tracking information from the pressure data. Moreover, since the linear pressure sensor array used in this study approximates the function of a specific subsection of the biological lateral line, it was necessary to determine whether further information (such as velocity sensors or out-of-plane pressure sensors) would benefit the estimation task. Deriving combined insight from statistical estimation theory, theoretical fluid dynamics, and fish studies, this work has taken a primarily experimental approach towards examining these questions. While in many cases these experiments were not the first to examine the sig- 
nals on a lateral-line-like array from and object or vortex stimulus (see for example Mogdans [52]), there are few if any precedents for utilizing the signals to estimate the original stimulus (the closest being the numerical study by Suzuki [74]).

The following section describes the principal contributions of this thesis for each chapter. This includes observations from the experiments as well as a successful demonstration of the wealth of the information achievable and of the limitations of this sensing approach. As this is the first major study using a pressure-based lateral-line-like sensor array to examine reproducing hypothesized abilities of the biological lateral line, this work opens a large set of further questions to be studied. The final section contains recommendations for future work, including a discussion of improvements and refinements to the current studies, as well as a discussion of topics that build from the results of this study.

\subsection{Principal contributions of the thesis}

\subsubsection{Passive cylinder tracking}

The study in passively tracking a cylinder utilized a data set generated by passing a round cylinder at constant velocity ranging between $0.2-0.5 \mathrm{~m} / \mathrm{s}$ past a linear array of seven pressure sensors embedded in a flat plate, passing at a distance between $2-20 \mathrm{~mm}$ from the array. The cylinder radius, position, and velocity were tracked using a particle filter as the nonlinear Kalman filter variants were shown to be inadequate. In order to tackle the problem inversion, an approximate analytic forward model was identified through comparisons against numerical viscous simulations. The pressure distribution about a cylinder in a steady flow is a well-known case and is a common validation tool for numerical simulation codes $[48,12]$, but the question of identifying the cylinder position and size from a small subset of the pressure field has never been previously addressed.

\section{Characterization of the relative state estimate accuracy}

The cylinder tracking (size and position) based on a linear pressure array was successfully accomplished using a particle filter that included a joint distribution for the dependent ra- 
dius and distance variables. The nature of the estimates, and in particular the error in the estimates, varies for each state variable. The $x$ position estimate, parallel to the linear sensor array, varies about the true value but shows little consistent bias. In contrast, the $y$ position estimates, perpendicular to the linear sensor array, consistently underestimate the distance between the sensors and the cylinder. In some cases the radius is also underestimated, though to a less extent than the $y$ position. This seems to occur only when the cylinder is very close to the sensors. This error behavior is likely particular to the analytic forward model being used. However, for the purposes of collision avoidance, the bias in the $y$ position is not of significant concern as it always works to increase the safety factor.

\section{Evaluation of tracking dependence on distance and speed}

The results of the cylinder tracking were examined for dependence on distance and speed of the cylinder, using a large series of experiments. The trends in estimation error are surprisingly mild in most cases. Although the experiments ranged from an effective pressure signal magnitude of $150 \mathrm{~Pa}$ to less than $10 \mathrm{~Pa}$, many of the cylinder variables did not show a noticeable dependence on these parameters. In the distant limit of small velocities and large distances, the measurements available to the sensor array approach the noise floor of the sensors and the inverse problem becomes insolvable. Based on the estimation results, the radius estimate appears to degrade for very small distances, but be roughly independent of velocity. The only other noticeable trends deal with the $x$ position estimate, which appears to worsen steadily but slowly with increasing velocity and distance.

\section{Velocity information is not necessary for tracking a cylinder}

The successful tracking of the moving cylinder based solely on the pressure from a linear array of seven sensors indicates that augmentation with velocity data is unnecessary. The specific problem scenario of the cylinder moving in still water is one in which velocity measurements would not be at a disadvantage (there is no steady flow to saturate the sensors), but there is no indication that their inclusion would add anything beyond redundancy to the flow measurements. In this experimental scenario, the direction of flow and motion is strongly encoded in both the asymmetry of the pressure distribution and it's translation. 


\section{Importance of Pressure differences between adjacent sensors}

While the pressure sensor array used to track a moving cylinder measures the pressure along a line with respect to a fixed (atmospheric) reference, the estimation results in this experiment, and in the others of this thesis, were impossible without considering the difference between pressure sensors and removing the fixed reference point. Although this removes some information, primarily about the depth of the sensors, it also removes significant common-mode drift that occurs due to influences of the surface. In the laboratory environment, large wavelength standing waves of the free surface with an amplitude of a few millimeters are a regular occurrence (excitable after a single cylinder pass) and generate oscillations in all the pressure sensors on the order of $10 \mathrm{~Pa}$. While standing waves may not be a common occurrence in an open environment, large wavelength surface oscillations of even larger amplitude are likely and would cause similar problems for absolute pressure measurements in shallow water. It is likely not coincidental that the fish lateral line canal system responds to pressure differences across adjacent pores in the canal.

\subsubsection{Passive cylinder shape classification}

In order to examine the ability to identify the shape of a moving cylinder that has passed a linear array of pressure sensors, experiments were done to test the ability to accurately classify trials from two cylinders of two similar cross sections. Cylinders with square and round cross sections with 5.1 and $7.6 \mathrm{~cm}$ diameters, velocities of 0.5 and $0.75 \mathrm{~m} / \mathrm{s}$, and separations from the sensors of 0.5 and $1.3 \mathrm{~cm}$. The pressure sensor array consisted of four sensors spaced horizontally 2.5 inches apart $(6.4 \mathrm{~cm})$. In total, data from 1000 passes were collected in order to closely examine the average differences and individual variation between trials. Two classification tests were developed, with one based on features from a principal component analysis trained on a data subset outperforming the alternative based on average differences. The results establish that certain similar shapes can be distinguished via a linear pressure sensor array, but also characterize a class of shapes that cannot be differentiated. 


\section{Average pressure response features poorly discriminate cylinder shapes}

Substantial variation in the data was observed across repeated trials of cylinders passing a streamlined body. This variation was almost entirely restricted to the period following the transition from high to low pressure which roughly corresponds to the leading edge of the cylinder. Ahead of this transition, the pressure increases steadily in a near-exponential ramp. After the transition, a large dip in the pressure is followed by a generally oscillatory (but not sinusoidal) recovery to the far field pressure. Repeated trials differ in particular with respect to these oscillations. Distinct differences between the two tested cylinder shapes are visible between the averaged pressure responses, with the most dramatic differences occurring after the pressure transition. A simple set of features based on these average differences results in a rate of approximately $10 \%$ misclassification. In many cases, this classification test is unable to overcome the natural variation in the data.

Pressure dominated by the wake substantially contributes to discriminating cylinder shapes

Shape classification based on principal component analysis features resulted in a high success rate for classifying a moving cylinder with square or round cross section (98.8\%). An analysis of the decision criterion determined that the pressure measured directly behind cylinder and thus alongside the wake is the second most informative region of the pressure response for distinguishing the shapes. The most informative region corresponds to the area near the point of minimum pressure, which matches the substantial differences in the average pressure responses in this region. The relative importance of the wake region is surprising, as the wake was primarily expected to obscure information about the shape due to the separation of the flow from the moving cylinder. While the range in sizes tested rules out vortex spacing being a factor, it is possible that the wake width, affected by the location and angle of flow separation, is the vehicle for information. 


\section{Shape classification is robust to variations in size, distance, and speed}

The decision criteria for classifying the shape of a cylinder after it has passed a linear array of pressure sensors were tested against cylinders of different sizes, separations from the sensors, and speeds. As mentioned, the decision criterion based on features from a principal component analysis was highly successful in distinguishing the shape of the cylinders regardless of the experimental parameters, indicating that the criterion is resilient against changes in parameters other than shape. For extreme changes in the size and distance the performance is certain to degrade. The combination of sizes, speeds, and distances is critical in establishing a criterion that is truly based on shape as opposed to other differences between experimental trials, as well as for establishing the robustness of the criterion.

\section{Velocity information is not necessary for distinguishing cylinder shapes}

As with tracking the cylinder position and size, there is no indication that concurrent velocity measurements would improve shape classification and identification. The decision criterion based on a principal component analysis already classifies $99 \%$ of the test cases correctly.

\subsubsection{Vortex tracking}

The ability to track vortices using a linear array of pressure sensors, emulating a lateral line, was examined from the perspective of a small streamlined body which roughly models a fish or small AUV, and from a flat plate which is a closer approximation to the hull of larger vehicles. The experiments with the streamlined body used a linear array of four pressure sensors spaced at 2.5 in $(6.4 \mathrm{~cm})$ to track the nearest vortex of a vortex pair generated by a swiveled foil. With seven sensors embedded on a flat plate $(0.75$ in. separation, $1.9 \mathrm{~cm})$, the experiments consisted of a single vortex passing in front of the array, separated from the initial vortex pair generated at a distance. 


\section{Estimation of vortex position and strength from a small body}

It was shown that with a linear pressure sensor array embedded in a small streamlined (fish-like) body can track the position and strength of the nearest vortex accurately. The two dimensional line vortex pair model was found sufficient for tracking the nearest vortex, with any extra unmodeled vortices or external fluid effects being included as Gaussian noise in the state transition model. The vortex tracking itself used a Kalman filter implementation taking advantage of the Gaussian noise model.

\section{Analysis of estimation with vortex-body interactions}

The experimental results from tracking a single vortex in the proximity of a flat plate represent an example of the behavior when the vortex-body interactions are not negligible. The induced velocity on the vortex itself is taken into account via a modification of the vortex model, leading to successful tracking overall. However, the proximity of the vortex and body leads to viscous effects that are not modeled: the development of secondary vorticity from the boundary layer on the wall. These secondary vortices form near the sensor array and are shed into the flow, causing perturbations to the measurements that are not accounted for in the vortex model. The result leads to extra variability in the distance estimate for the primary vortex, without substantially affecting the estimate of its position parallel to the sensor array.

\section{Sensor array design for vortex tracking}

Based on an examination of the estimation of a simulated vortex or vortex pair at a single time instant, relationships between the range of the vortices of interest and the pressure sensor array length and density were established. In both cases of a vortex pair near a streamlined body and a single vortex near a flat plate, the array length maximum distance for tracking a vortex was found to be tied to the array length, with the array length as roughly twice the vortex distance. Similarly, the closest distance for resolving the position of a vortex was connected to the sensor density, with a minimum spacing of approximately twice the minimum distance. For arrays with parameters outside these specifications, the 
resolution of the position of the vortex degrades, leading to increased convergence times and possible bias, but not into a complete inability to estimate the vortex. In the case of the array length, it should be emphasized that the relationship serves as the minimum necessary for tracking a vortex up to a given distance.

\section{Velocity information is not necessary for tracking vortices, but may be beneficial}

As in the case of the cylinder tracking and identification, the analysis of the vortex tracking successfully achieved its goal without requiring extra velocity measurements. However, unlike the other cases, it may be beneficial to augment the pressure array with velocity measurements. The pressure field due to a vortex pair is symmetric in front and behind the position of the vortex. The sign of the vortex strength determines the direction of motion between measurements, and thus was able to be estimated successfully from the pressure data, but at a single instant in time the sign of the vortex strength is unresolvable based on pressure. Augmenting the data set by a reliable velocity measurement indicating the direction of fluid motion would likely result in much faster convergence, particularly for the vortex strength, but is not necessary for vortex tracking.

\subsection{Recommendations for future work}

This study is the first description of inference and signal processing with a pressure-based lateral-line-like array that is not focused on detecting an oscillating dipole. While it is always possible to improve the experimental setups and equipment, the successful results of this study open up many avenues of further applications of a lateral-line-like sensor array for AUV's and ocean vehicles. Given that the fish lateral line is still an active area of research, the results of the current study also suggest behaviors of the lateral line that can be tested in order to further its understanding. 


\subsubsection{Improved sensor array density for multiple vortex tracking}

In the vortex pair tracking experiments and estimation results, it was found at that an accurate estimate of the state of the nearest vortex was available but that the low sensitivity of the pressure measurements to the second, further, vortex made it difficult to track. These experiments used a linear array of four pressure sensors spaced 2.5 in $(6.4 \mathrm{~cm})$ apart. A high-density array of sufficient length for the distance of the second vortex would likely result in an improved estimate of the second vortex due to the increased reliability of the measurements. High density arrays of pressure sensors are difficult to achieve (but not unprecedented [36]) using off-the-shelf pressure sensors as was done in this study, but would be feasible if constructed directly with MEMS technology [35].

\subsubsection{Vortex street tracking}

The study in tracking vortices from a small streamlined body demonstrated that it was possible to track the state of the closest vortex in a vortex pair. In natural environments, vortices are frequently found in an alternating series spaced with regular intervals, as the vortex streets forming behind blunt flow obstructions or oscillating thrust-producing foils. The study of vortex tracking based on a lateral-line-like array could be straightforwardly extended to examine the ability to track individual vortices in such a regular vortex street. Although multiple vortices would be present, their spacing would distribute them along the length of the arrays and likely not lead to the same ambiguities as for the vortex pair.

\subsubsection{Numerical forward models for wakes}

The potential flow models discussed in Chapter 3 for tracking a moving cylinder have proved adequate for the task. However, the resulting state estimates exhibited variability during individual runs, possibly due to the steady analytic approximation to the wake being used. In order to improve the forward model, a future approach may directly utilize approximate real-time numerical models such as those by Weymouth [83]. 


\subsubsection{Biological object discrimination}

Based on the results of the study on cylinder shape identification, it is likely that there is a class of object shapes which would not be distinguishable when moving past a lateral line or similarly inspired sensor. These similar shapes would have similar wake widths based on the size and bluntness of their forward faces. This suggests a very simple test along the lines of the experiments by Mogdans and Bleckmann [52], in which the hypothesis can be tested with live fish.

\subsubsection{Active object identification}

The cylinder tracking study described in this thesis corresponds to the passive object identification scenario, in which the interaction between the cylinder and a steady fluid flow externally generate the pressure field signal. This scenario corresponds to some potential AUV situations, but is also useful as a test case for object detection since the amplitude of stimulus can be easily manipulated. In contrast, the active object identification scenario, in which the fluid flow is driven purely by the motion of the sensing platform itself, presents a less favorable noise environment due to the reduced signal amplitude and increased vibrations of the sensors. Despite the increased measurement difficulty this sort of object detection and identification would be of great benefit to an AUV, which would not be dependent on an external flow to interrogate an object of interest.

\subsubsection{Two-dimensional sensor arrays}

Particularly in the case of object detection and identification, current MEMS technology makes it possible to use regular dense two-dimensional arrays of pressure sensors to map the pressure distribution over a surface instead of the linear array considered in this study. This additional information provides the opportunity to extend the inference beyond twodimensional cross sections of objects as was considered in the present study. This adds considerable complication to the problem, but in conjunction with the active object identification could lead to forming complete three dimensional maps of arbitrary unknown objects, making it the ultimate goal of touch-at-a-distance lateral-line-like sensors. 


\subsubsection{Local flow models for large objects}

The present discussion of cylinder tracking and identification via a distributed linear pressure sensor array is applicable to cross sections with length scales on the order of the length of the array. In contrast, particularly for applications in navigation and maneuvering, it would also be beneficial to extend the object identification studies to objects with much larger length scales. In this regime, it would not necessarily be preferable to attempt to obtain or model a full description of the object. In the very large extreme, for example, a small vehicle searching for mines on a tanker hull would not benefit greatly from having a flow model around the entire tanker as long as it had an adequate local model. Extending the object identification to very large objects with local perturbations would likely require a completely new approach to the forward model. One concern with local modeling approaches is the possible ambiguity of the fluid-solid boundary. As an initial note on resolving this ambiguity, it should be noted that fish have been observed to periodically make contact with walls using their pectoral fins [85], which may be the biological approach to dealing with this issue. 


\section{Bibliography}

[1] S. A. Ansari, N. Phillips, G. Stabler, P. C. Wilkins, R. Zbikowski, and K. Knowles. Experimental investigation of some aspects of insect-like flapping flight aerodynamics for application to micro air vehicles. In G. K. Taylor, M. S. Triantafyllou, and C. Tropea, editors, Animal Locomotion, pages 215-236. Springer, 2010.

[2] C. F. Baker and J. C. Montgomery. The sensory basis of rheotaxis in the blind mexican cave fish, astyanax fasciatus. Journal of Comparative Physiology A-Sensory Neural and Behavioral, 184(5):519-527, 1999.

[3] G. K. Batchelor. An introduction to fluid dynamics. Cambridge University Press, New York, NY, 2000.

[4] H. Bleckmann, G. Tittel, and E. Blubaum-Gronau. The lateral line system of surfacefeeding fish: anatomy, physiology, and behavior. In S. Coombs, P. Gorner, and H. Munz, editors, The mechanosensory lateral line: Neurobiology and evolution, pages 501-526. Springer, 1989.

[5] R. D. Blevins. Flow-Induced Vibration. Van Nostrand Reinhold, New York, NY, 1990.

[6] R. Bouffanais, G. D. Weymouth, and D. K. P. Yue. Hydrodynamic object recognition using pressure sensing. Proceedings of the Royal Society A: Mathematical, Physical and Engineering Science, 2010. 
[7] C. B. Braun and S. Coombs. The overlapping roles of the inner ear and lateral line: the active space of dipole source detection. Philosophical Transactions of the Royal Society of London, 355:1115-1119, 2000.

[8] T. Burt de Perera. Spatial parameters encoded in the spatial map of the blind mexican cave fish, astyanax fasciatus. Animal Behaviour, 68(2):291-295, 2004.

[9] L. W. Carr. Progress in analysis and prediction of dynamic stall. Journal of Aircraft, 25(21):6-17, 1987.

[10] B. P. Chagnaud, H. Bleckmann, and M. H. Hofmann. Kármán vortex street detection by the lateral line. Journal of Comparative Physiology A, 193(7):753-763, 2007.

[11] B. P. Chagnaud, M. H. Hofmann, and J. Mogdans. Responses to dipole stimuli of anterior lateral line nerve fibres in goldfish, carassius auratus, under still and running water conditions. Journal of Comparative Physiology A, 193(2):249-263, 2006.

[12] N.R. Clarke and O.R. Tutty. Construction and validation of a discrete vortex method for the two-dimensional incompressible navier-stokes equations. Computers \& Fluids, 23(6):751 - 783, 1994.

[13] S. Coombs. Smart skins: Information processing by lateral line flow sensors. $A u$ tonomous Robotics, 11:255-261, 2001.

[14] S. Coombs, C. B. Braun, and B. Donovan. The orienting response of lake michigan mottled sculpin is mediated by canal neuromasts. Journal of Experimental Biology, 204(2):337 -348, 2001.

[15] S Coombs and R. A. Conley. Dipole source localization by mottled sculpin. i. approach strategies. Journal of Comparative Physiology A: Neuroethology, Sensory, Neural, and Behavioral Physiology, 180(4):387-399, 1997.

[16] S. Coombs and R. R. Fay. Dipole source localization by mottled sculpin, Cottus bairdi. Journal of the Acoustical Society of America, 93:2116-2123, 1993. 
[17] S. Coombs, J. J. Finneran, and R. A. Conley. Hydrodynamic image formation by the peripheral lateral line system of the lake michigan mottled sculpin, cottus bairdi. Philosophical Transactions: Biological Sciences, 355(1401):1111-1114, 2000.

[18] S. Coombs and J. Janssen. Behavioral and neurophysiological assessment of lateral line sensitivity in the mottled sculpin, cottus bairdi. Journal of Comparative Physiology A, 167(4), 1990.

[19] S. Coombs, J. Janssen, and J. C. Montgomery. Functional and evolutionary implications of peripheral diversity in lateral line systems. In D. B. Webster, R. R. Fay, and A. N. Popper, editors, The evolutionary biology of hearing, pages 267-294. Springer, New York, NY, 1992.

[20] S. Coombs and P. Patton. Lateral line stimulation patterns and prey orienting behavior in the lake michigan mottled sculpin (Cottus bairdi). Journal of Comparative Physiology A: Neuroethology, Sensory, Neural, and Behavioral Physiology, 195(3):279-297, 2009.

[21] B. Curcic-Blake and S. M. van Netten. Source location encoding in the fish lateral line canal. Journal of Experimental Biology, 209(8):1548-1559, 2006.

[22] G. Dehnhardt, B. Mauck, W. Hanke, and H. Bleckmann. Hydrodynamic TrailFollowing in harbor seals (Phoca vitulina). Science, 293(5527):102 -104, July 2001.

[23] E. J. Denton and J. Gray. Mechanical factors in the excitation of clupeid lateral lines. Proceedings of the royal society of London series B-Biological sciences, 218(1210):1-26, 1983.

[24] S. Dijkgraaf. The functioning and significance of the lateral line organs. Biological Reviews, 38:51-105, 1963.

[25] J. Englemann, W. Hanke, J. Mogdans, and H. Bleckmann. Hydrodynamic stimuli and the fish lateral line. Nature, 408:51-52, 2000. 
[26] L. E. Ericsson and J. P. Reding. Fluid mechanics of dynamic stall: Part 1. unsteady flow concepts. Journal of Fluids and Structures, 2:1-33, 1989.

[27] A. Gelb, editor. Applied Optimal Estimation. MIT Press, Cambridge, Massachusetts, 1974.

[28] M. A. Gibbs. Lateral line receptors: Where do they come from developmentally and where is our research going? Brain, Behavior, and Evolution, 64:163-181, 2004.

[29] N. Glaeser, S. Wieskotten, C. Otter, G. Dehnhardt, and W. Hanke. Hydrodynamic trail following in a California sea lion (Zalophus californianus). Journal of Comparative Physiology A-Neuroethology: Sensory Neural and Behavioral Physiology, 197(2):141-151, 2011.

[30] R. Gopalkrishnan, M. S. Triantafyllou, G. S. Triantafyllou, and D. Barrett. Active vorticity control in a shear flow using a flapping foil. Journal of Fluid Mechanics, 274:1-21, 1994.

[31] W. Hanke, M. Witte, L. Miersch, M. Brede, J. Oeffner, M. Michael, F. Hanke, A. Leder, and G. Dehnhardt. Harbor seal vibrissa morphology suppresses vortexinduced vibrations. Journal of Experimental Biology, 213(15):2665 -2672, 2010.

[32] E. S. Hassan. On the discrimination of spatial intervals by the blind cave fish (Anoptichthys jordani). Journal of Comparative Physiology A, 159:701-710, 1986.

[33] E. S. Hassan. Mathematical description of the stimuli to the lateral line system of fish derived from a 3-dimensional field analysis: I. the cases of moving in open water and of gliding towards a plane surface. Biological Cybernetics, 66:443-452, 1992.

[34] E. S. Hassan. Mathematical description of the stimuli to the lateral line system of fish derived from a 3-dimensional field analysis: II. the case of gliding alongside or above a plane surface. Biological Cybernetics, 66:453-461, 1992.

[35] S.M. Hou, V.I. Fernandez, F.S. Hover, J. Lang, , and M.S. Triantafyllou. Development and application of distributed mems pressure sensor array for auv object avoidance. In 
Proceedings of the International Symposium on Unmanned Untethered Submersible Technology (UUST), August 2009.

[36] L. M. Hudy, A. M. Naguib, and W. M. Humphreys. Wall-pressure-array measurements beneath a separating/reattaching flow region. Physics of Fluids, 15(3):706-717, March 2003.

[37] Inc. Hydroid, 2011. http://www.hydroidinc.com/remus100.html.

[38] J. E. Jackson. A User's Guide to Principal Components. Wiley, Hoboken, New Jersey, 2003.

[39] J. Jeong and F. Hussain. On the identification of a vortex. Journal of Fluid Mechanics, 285:69-94, 1994.

[40] I. T. Jolliffe. Principal Component Analysis. Springer, New York, New York, second edition, 2002.

[41] S. J. Julier and J. K. Uhlmann. Unscented filtering and nonlinear estimation. Proceedings of the IEEE, 92(3):401-422, 2004.

[42] A. J. Kalmijn. Functional aspects of the evolution of the auditory system of actinopterygian fish. In J. Atema, R. R. Fay, A. N. Popper, and W. N Tavolga, editors, Sensory biology of aquatic animals, pages 83-130. Springer-Verlag, 1988.

[43] J. C. Liao. The role of the lateral line and vision on body kinematics and hydrodynamic preference of rainbow trout in turbulent flow. Journal of Experimental Biology, 209(20):4077-4090, 2006.

[44] J. C. Liao. A review of fish swimming mechanics and behaviour in altered flows. Philosophical Transactions of the Royal Society B - Biological Sciences, 362(1487):1973-1993, 2007.

[45] J. C. Liao, D. N. Beal, G. V. Lauder, and M. S. Triantafyllou. Fish exploiting vortices decrease muscle activity. Science, 302(5650):1566 -1569, 2003. 
[46] J. C. Liao, D. N. Beal, G. V. Lauder, and M. S. Triantafyllou. The Karman gait: novel body kinematics of rainbow trout swimming in a vortex street. Journal of Experimental Biology, 206(6):1059-1073, 2003.

[47] S. Licht, V. Polidoro, M. Flores, F. S. Hover, and M. S. Triantafyllou. Design and projected performance of a flapping foil auv. IEEE Journal of Oceanic Engineering, 29(3):786-794, 2004.

[48] K. Mahesh, G. Constantinescu, and P. Moin. A numerical method for large-eddy simulation in complex geometries. Journal of Computational Physics, 197(1):215$240,2004$.

[49] M. E. McConney, K. D. Anderson, L. L. Brott, R. R. Naik, and V. V. Tsukruk. Bioinspired material approaches to sensing. Advanced Functional Materials, 19(16):2527$2544,2009$.

[50] M. J. McHenry, K. E. Feitl, J. A. Strother, and W. J. Van Trump. Larval zebrafish rapidly sense the water flow of a predator's strike. Biology Letters, 5(4):477 -479, 2009.

[51] M. J. McHenry, J. A. Strother, and S. M. van Netten. Mechanical filtering by the boundary layer and fluid-structure interaction in the superficial neuromast of the fish lateral line system. Journal of Comparative Physiology A: Neuroethology, Sensory, Neural, and Behavioral Physiology, 194(9):795-810, 2008.

[52] J. Mogdans and H. Bleckmann. Responses of the goldfish trunk lateral line to moving objects. Journal of Comparative Physiology A: Neuroethology, Sensory, Neural, and Behavioral Physiology, 182(5):659-676, 1998.

[53] J. C. Montgomery, C. F. Baker, and A. G. Carton. The lateral line can mediate rheotaxis in fish. Nature, 389(6654):960-963, 1997.

[54] J. C. Montgomery and S. Coombs. Peripheral encoding of moving sources by the lateral line system of a sit-and-wait predator. Journal of Experimental Biology, 201(1):91-102, 1998. 
[55] J. C. Montgomery, S. Coombs, and C. F. Baker. The mechanosensory lateral line system of the hypogean form of Astyanax fasciatus. Evolutionary Biology of Fishes, 62:87-96, 2001.

[56] J. C. Montgomery, S. Coombs, and M. Halstead. Biology of the mechanosensory lateral line in fishes. Reviews in Fish Biology and Fisheries, 5(4):399-416, 1995.

[57] J. C. Montgomery and J. A. MacDonald. Sensory tuning of lateral line receptors in antartic fish to the movements of planktonic prey. Science, 235(4785):195-196, 1987.

[58] J. C. Montgomery, S. P. Windsor, and D. K. Bassett. Behavior and physiology of mechanoreception: separating signal and noise. Integrative Zoology, 4(1):3-12, 2009.

[59] H. Munz. Single unit activity in the peripheral lateral line system of the chichlid fish Sarotherodon niloticus 1. Journal of Comparative Physiology A, 157(5):555-568, 1986.

[60] J. N. Newman. Marine Hydrodynamics. MIT Press, Cambridge, Massachusetts, 1977.

[61] T. Perez and G. C. Goodwin. Constrained predictive control of ship fin stabilizers to prevent dynamic stall. Control Engineering Practice, 16:482-494, 2008.

[62] T. J. Pitcher, B. L. Partridge, and C. S. Wardle. A blind fish can school. Science, 194(4268):963 -965, 1976.

[63] K. Pohlmann, J. Atema, and T. Breithaupt. The importance of the lateral line in nocturnal predation of piscivorous catfish. Journal of Experimental Biology, 207:29712978, 2004.

[64] K. Pohlmann, F. W. Grasso, and T. Breithaupt. Tracking wakes: The nocturnal predatory strategy of piscivorous catfish. Proceedings of the National Academy of Sciences of the United States of America, 98(13):7371-7374, 2001.

[65] M. A. Rapo, H. Jiang, M. A. Grosenbaugh, and S. Coombs. Using computational fluid dynamics to calculate the stimulus to the lateral line of a fish in still water. Journal of Experimental, 212(10):1494-1505, 2009. 
[66] Z. Ren and K. Mohseni. A model of lateral line of fish for vortex sensing. In 40th Fluid Dynamics Conference and Exhibit. AIAA, 2010.

[67] B. Ristic, S. Arulampalam, and N. Gordon. Beyond the Kalman filter: Particle filters for tracking applications. Artech house, New York, NY, 2004.

[68] P. G. Saffman. Vortex Dynamics. Cambridge University Press, New York, NY, 1995.

[69] M. Satou, A. Shiraishi, T. Matsushima, and N. Okumoto. Vibrational communication during spawning behavior in the himé salmon (landlocked red salmon,Oncorhynchus nerka). Journal of Comparative Physiology A, 168(4):417-428, 1991.

[70] A. Sichert, R. Bamler, and J. van Hemmen. Hydrodynamic object recognition: When multipoles count. Physical Review Letters, 102(5), 2009.

[71] B. J. Simpson. Experimental studies of flapping foils for energy extraction. PhD thesis, Massachusetts Institute of Technology, 2009.

[72] K. Streitlien. Extracting energy from unsteady flows through vortex control. $\mathrm{PhD}$ thesis, Massachusetts Institute of Technology, 1994.

[73] K. Streitlien and M. S. Triantafyllou. Force and moment on a Joukowski profile in the presence of point vortices. American Institute of Aeronautics and Astronautics Journal, 33(4):603-610, 1995.

[74] T. Suzuki and T. Colonius. Inverse-imaging method for detection of a vortex in a channel. American Institute of Aeronautics and Astronautics Journal, 41:1743-1751, 2003.

[75] T. Teyke. Collision with and avoidance of obstacles by blind cave fish Anoptichthys jordani (characidae). Journal of Comparative Physiology A, 157:837-843, 1985.

[76] T. Teyke. Learning and remembering the environment in the blind cave fish anoptichthys jordani. Journal of Comparative Physiology A: Neuroethology, Sensory, Neural, and Behavioral Physiology, 164(5):655-662, 1989. 
[77] T. Teyke. Morphological differences in the neuromasts of the blind cave fish Astyanax Hubbsi and the sighted river fish Astyanax Mexicanus. Brain Behavior and Evolution, 35(1):23-30, 1990.

[78] S. Theodoridis and K. Koutroumbas. Pattern Recognition. Academic Press, San Diego, California, third edition, 2006.

[79] S. M. van Netten. Hydrodynamic detection by cupulae in a lateral line canal: Functional relations between physics and physiology. Biological Cybernetics, 94:67-85, 2006.

[80] D. Vogel and H. Bleckmann. Behavioral discrimination of water motions caused by moving objects. Journal of Comparative Physiology A, 186:1107-1117, 2000.

[81] H. Vollmers. Detection of vortices and quantative evaluation of their main parameters from experimental velocity data. Measurement Science and Technology, 12:11991207, 2001.

[82] R. Weissert and C. von Campenhausen. Discrimination between stationary objects by the blind cave fish Anoptichthys jordani (characidae). Journal of Comparative Physiology A, 143:375-381, 1981.

[83] G. Weymouth, 2011. http://web.mit.edu/weymouth/www/.

[84] S. P. Windsor and M. J. McHenry. The influence of viscous hydrodynamics on the fish lateral-line system. Integrative and Comparative Biology, 49(6):691-701, 2009.

[85] SP Windsor, D Tan, and JC Montgomery. Swimming kinematics and hydrodynamic imaging in the blind mexican cave fish (Astyanax fasciatus). Journal of Experimental Biology, 211(18):2950-2959, 2008.

[86] Malima I. Wolf, Stephen C. Licht, Franz Hover, and Michael S. Triantafyllou. Open loop swimming performance of 'Finnegan' the Biomimetic Flapping Foil AUV. In Wong, P. C. and Uchida, K. and Chung, J. S. and Sayed, M. and Moshagen, H. and Fontaine, E., editor, Proceedings of the Sixteenth (2006) International Offshore and 
Polar Engineering Conference, Vol. 2, International Offshore and Polar Engineering Conference Proceedings, pages 247-253, 2006.

[87] Y. Yang, J. Chen, J. Engel, S. Pandya, N. Chen, C. Tucker, S. Coombs, and C. Liu. Distant touch hydrodynamic imaging with an artificial lateral line. Proceedings of the National Academy of Science, 103(50):18891-18895, 2006.

[88] Y. C. Yang, N. Chen, C. Tucker, S. Pandya, D. Jones, and C. Liu. Biomimetic flow sensing using artificial lateral lines. Proceedings of the ASME International Mechanical Engineering Congress, pages 1331-1338, 2008.

[89] Q. Zhu, M. Haase, and C. H. Wu. Modeling the capacity of a novel flow-energy harvester. Applied Mathematical Modelling, 33(5):2207-2217, 2009. 